s

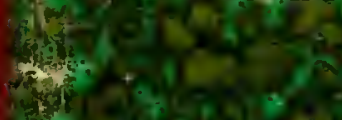

40

$-4+2 ; x^{2}$

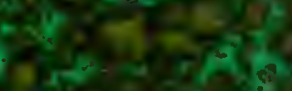

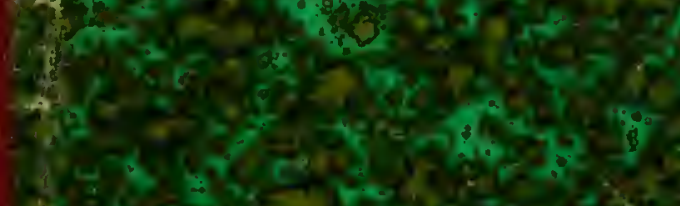

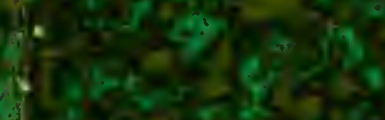

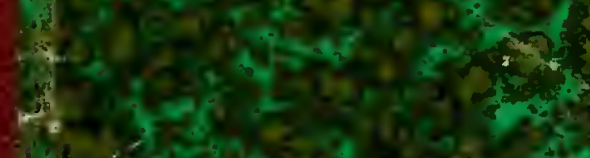

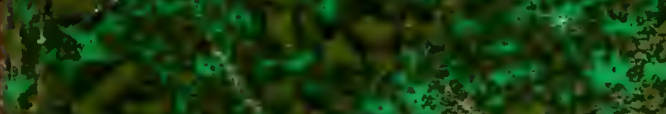

(f)

6. $-x+2,8$

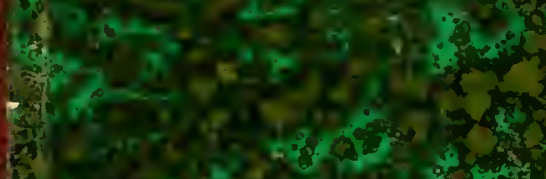

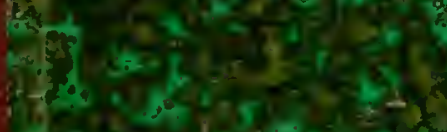

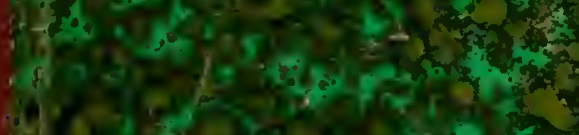

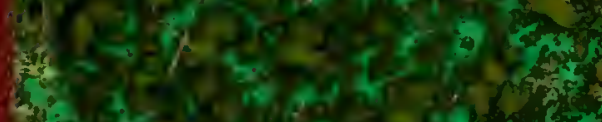

6.

Fin

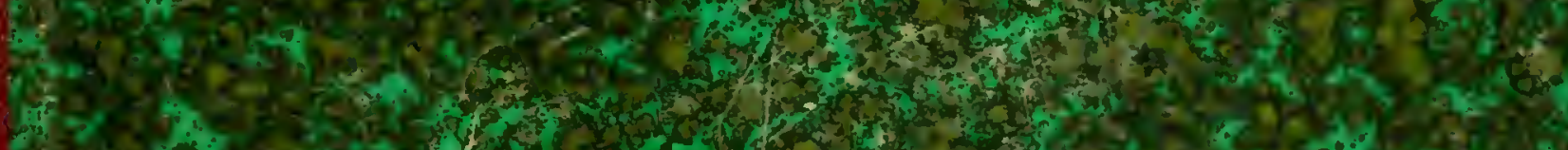

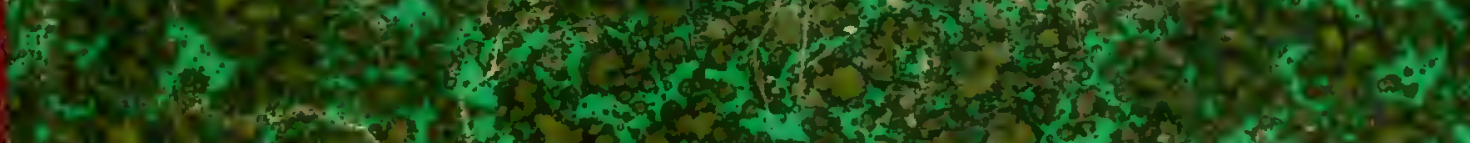

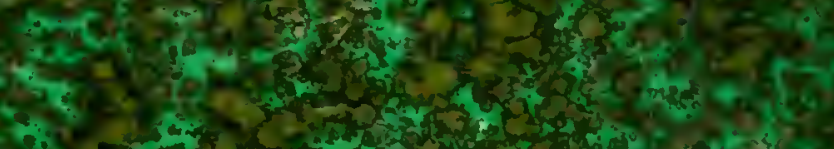
entions

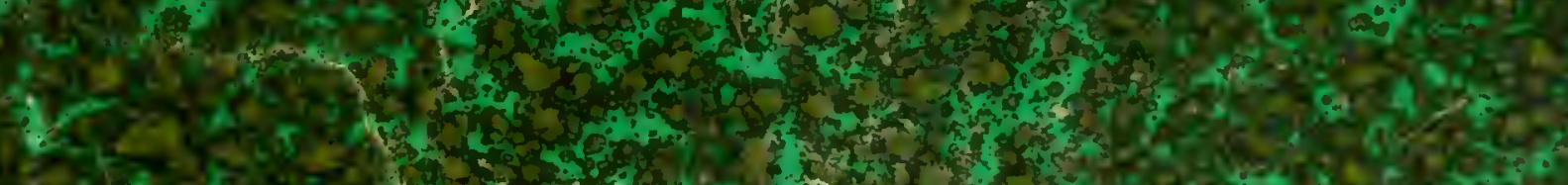

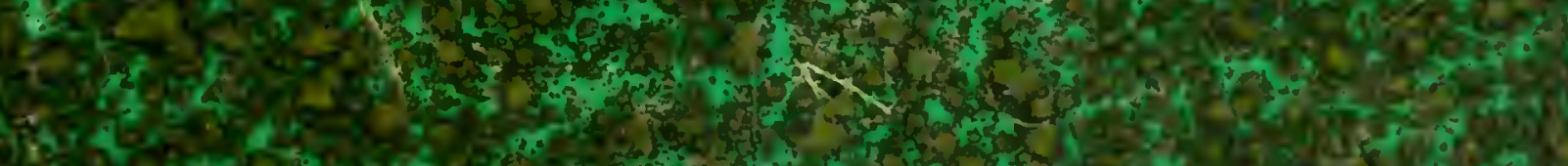

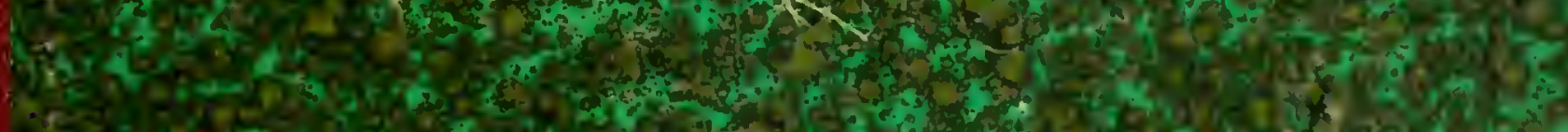

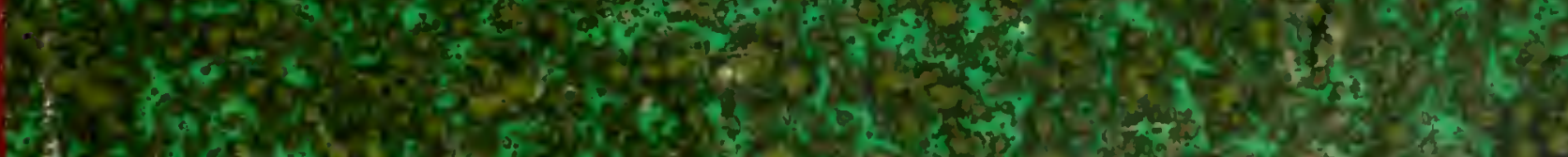

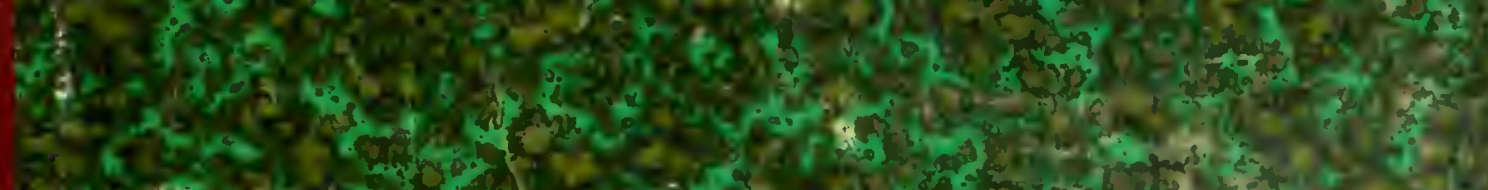

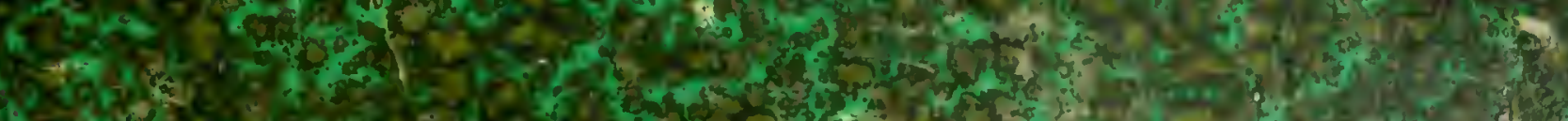

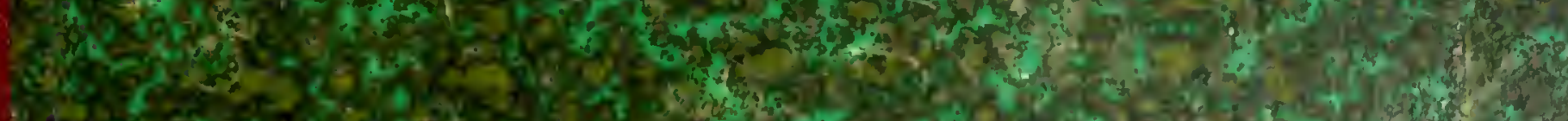

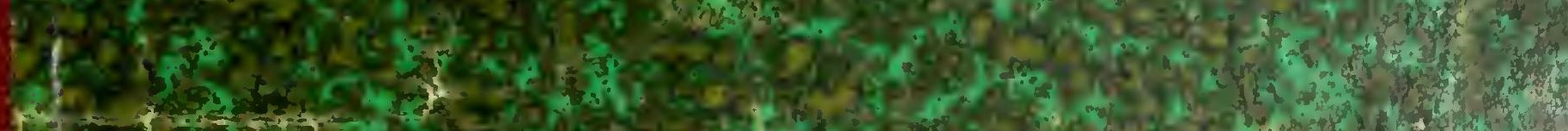

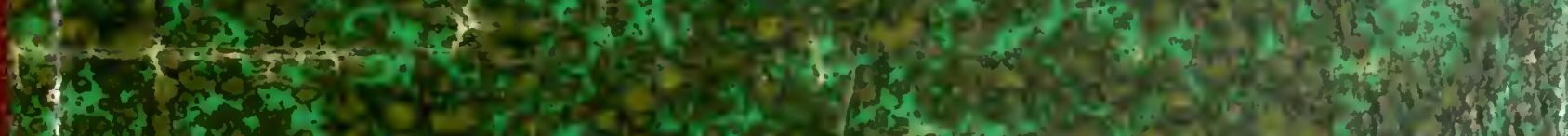
thes

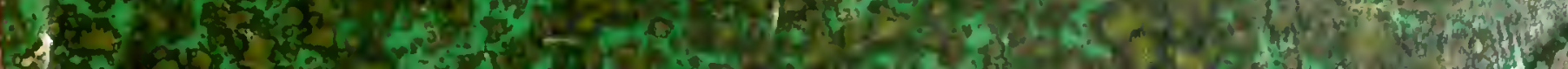




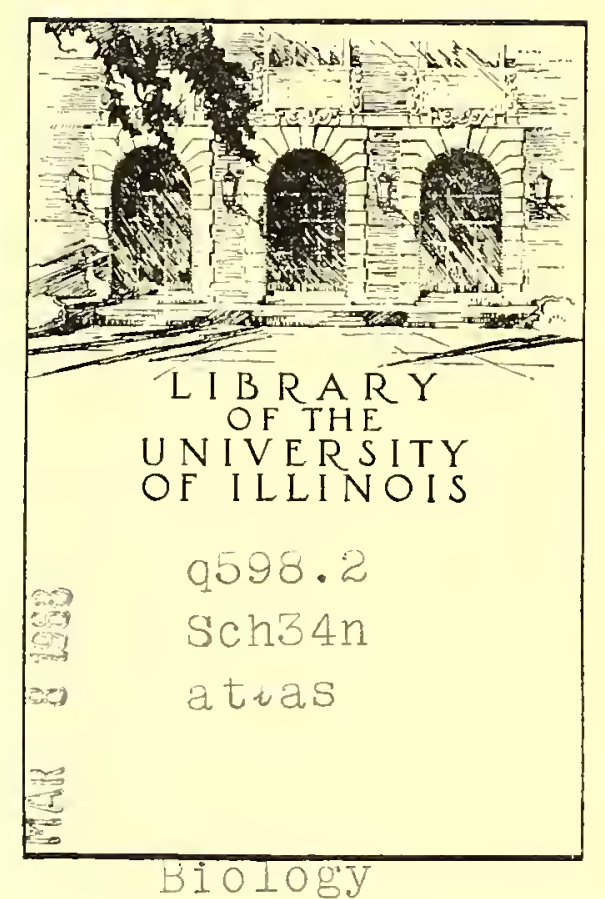


Digitized by the Internet Archive in 2011 with funding from

University of Illinois Urbana-Champaign 



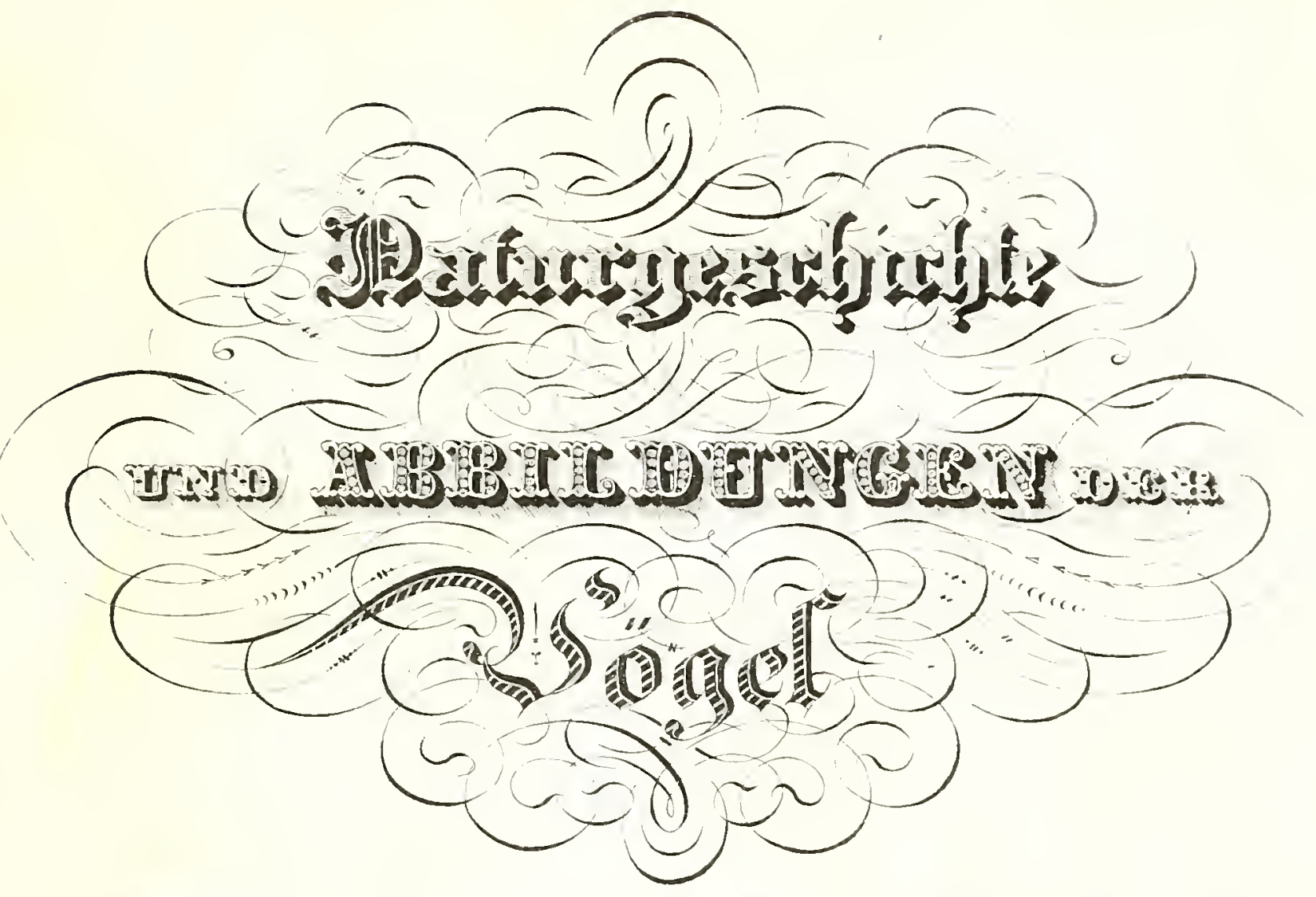

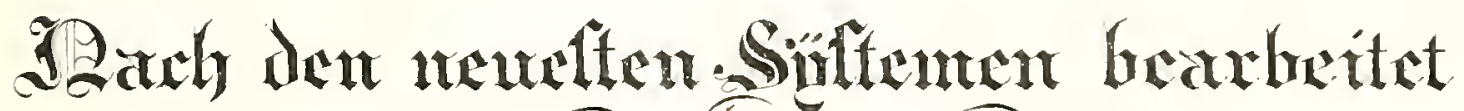

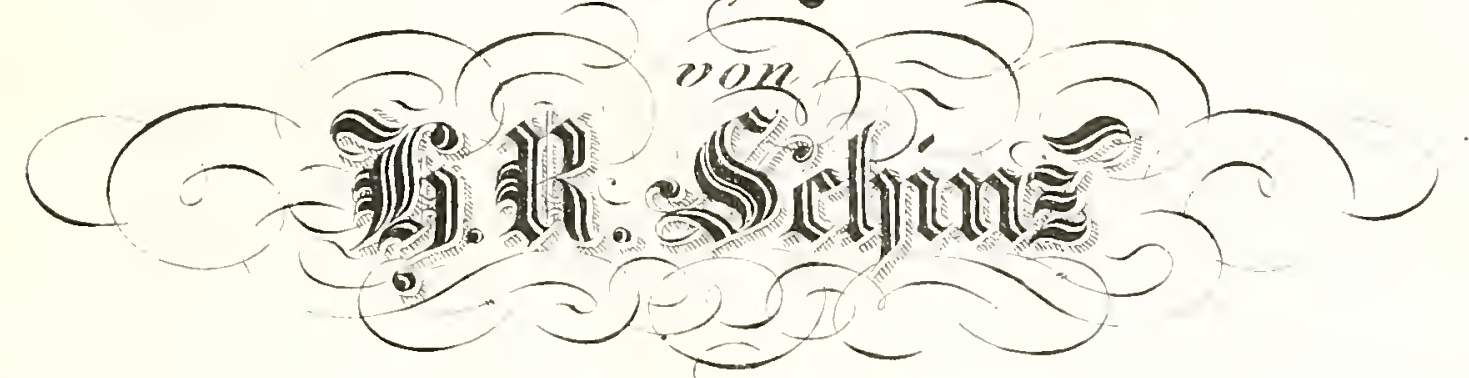

Med. Dr. Lehrer der Naturogeschichte u. Mitolied melurerer gelehrten Gesellschaften.

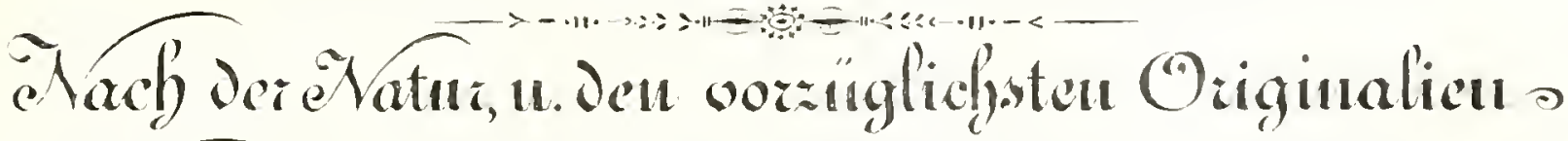

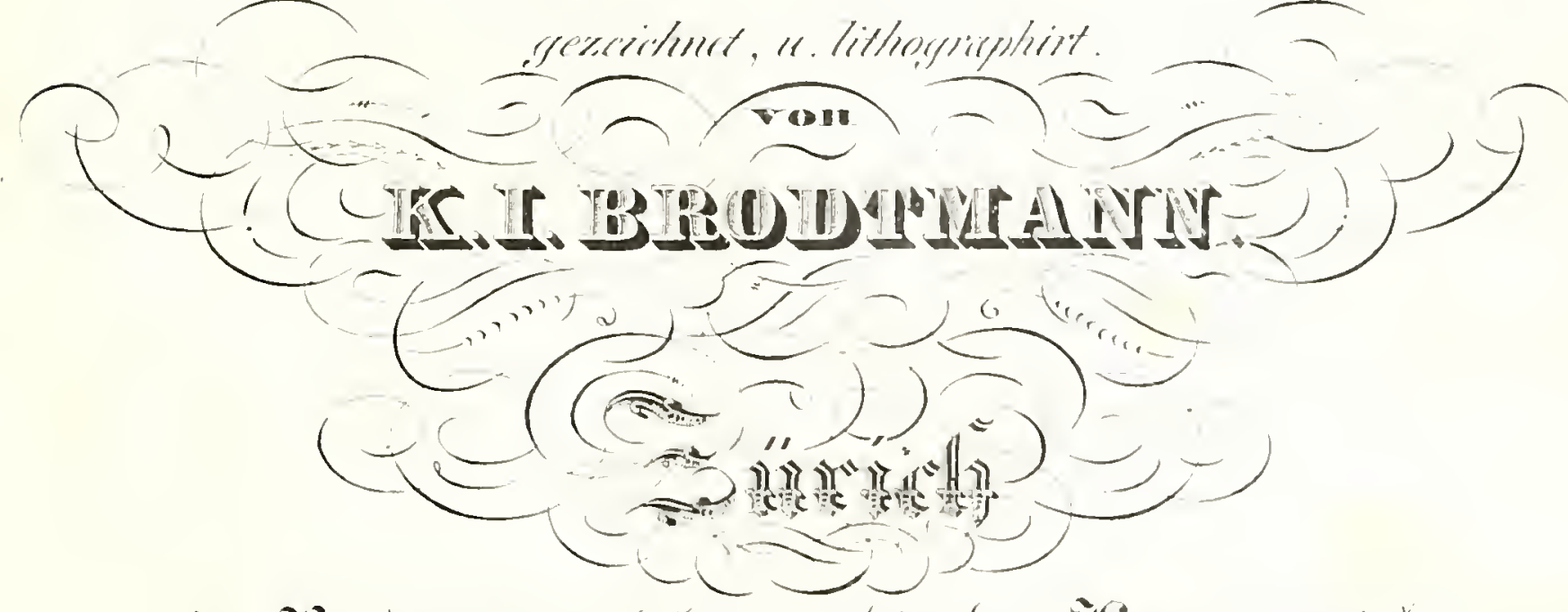

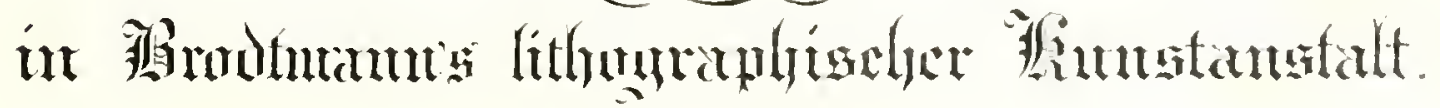



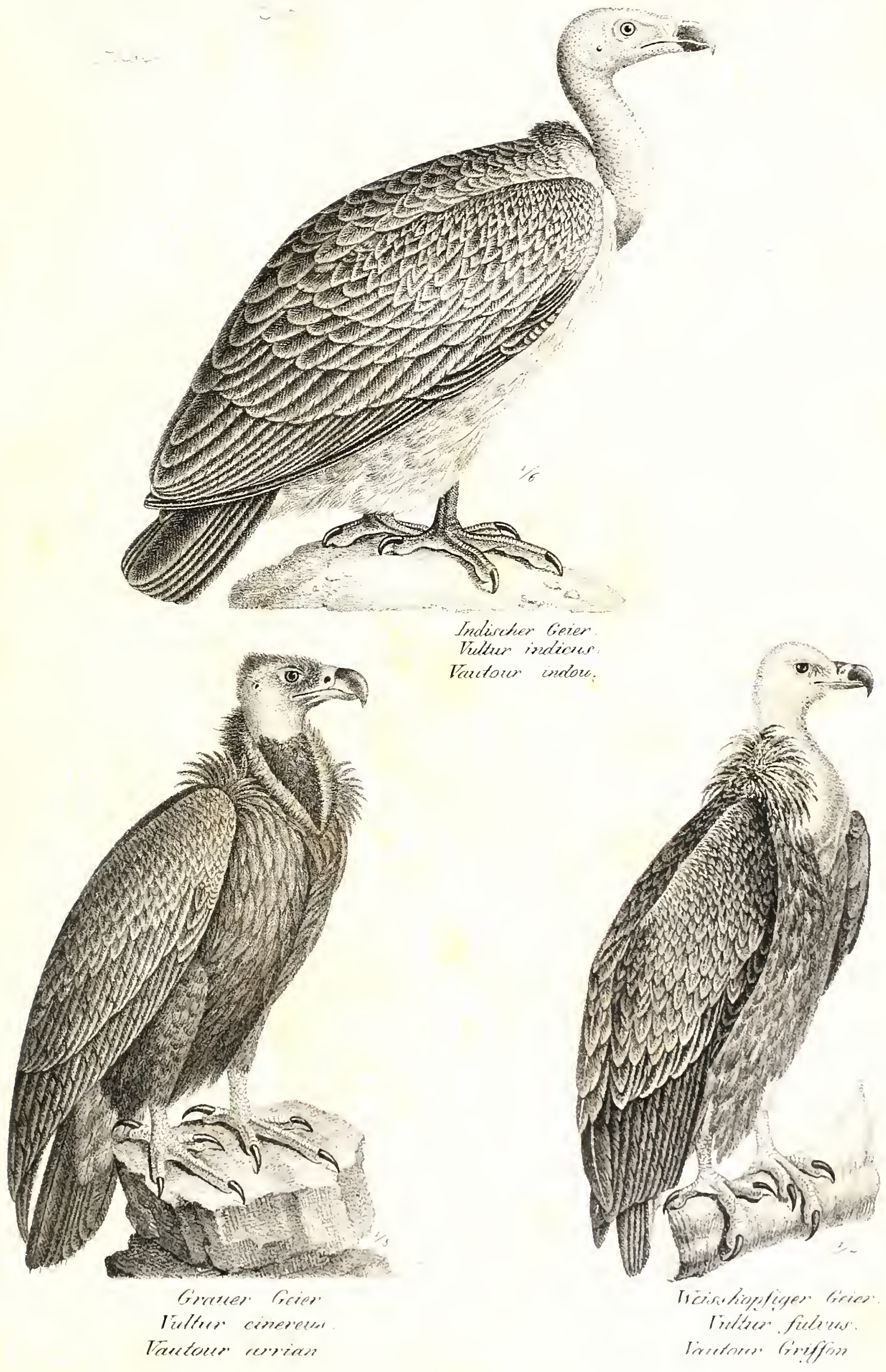


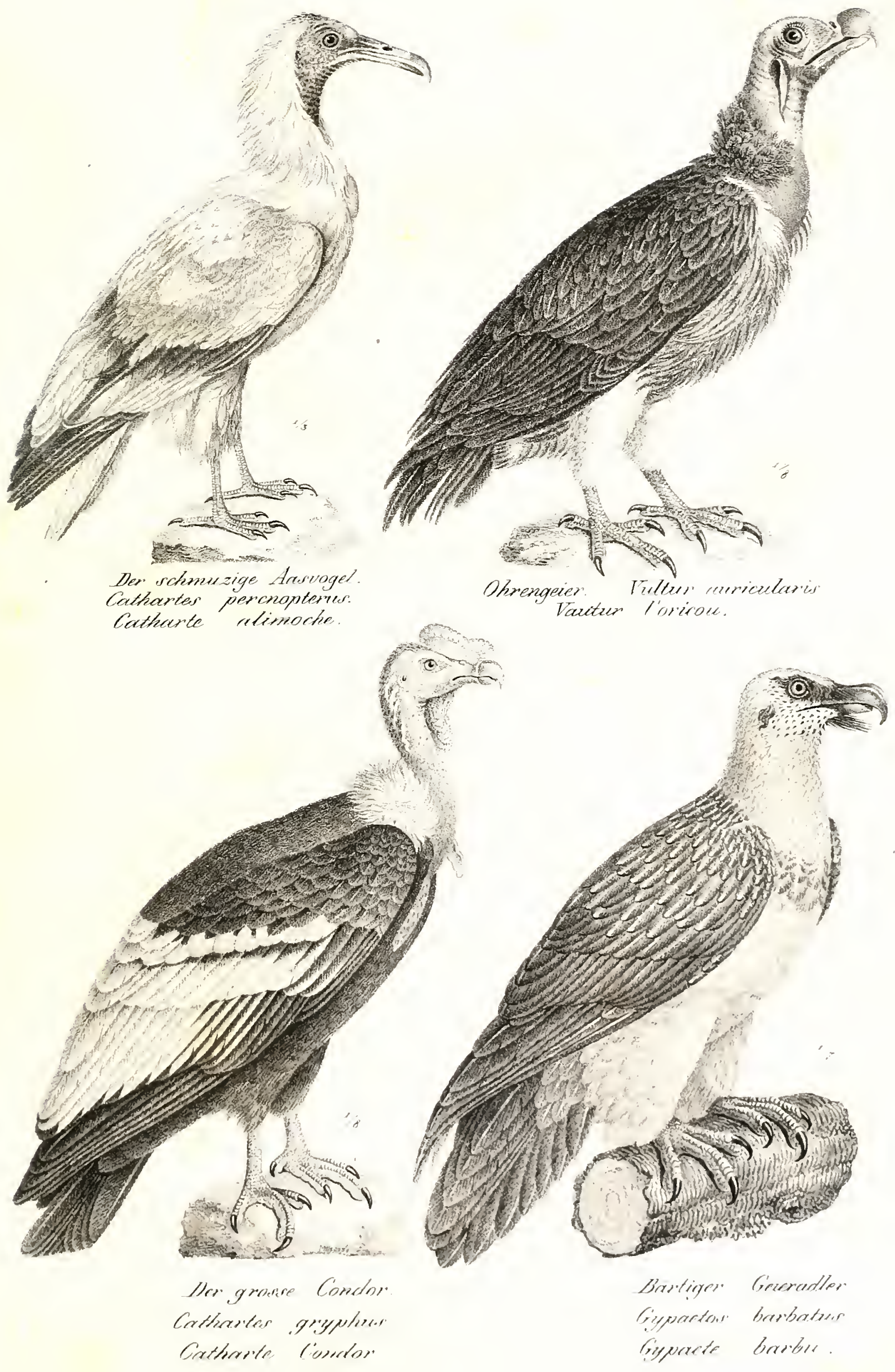

$p=$

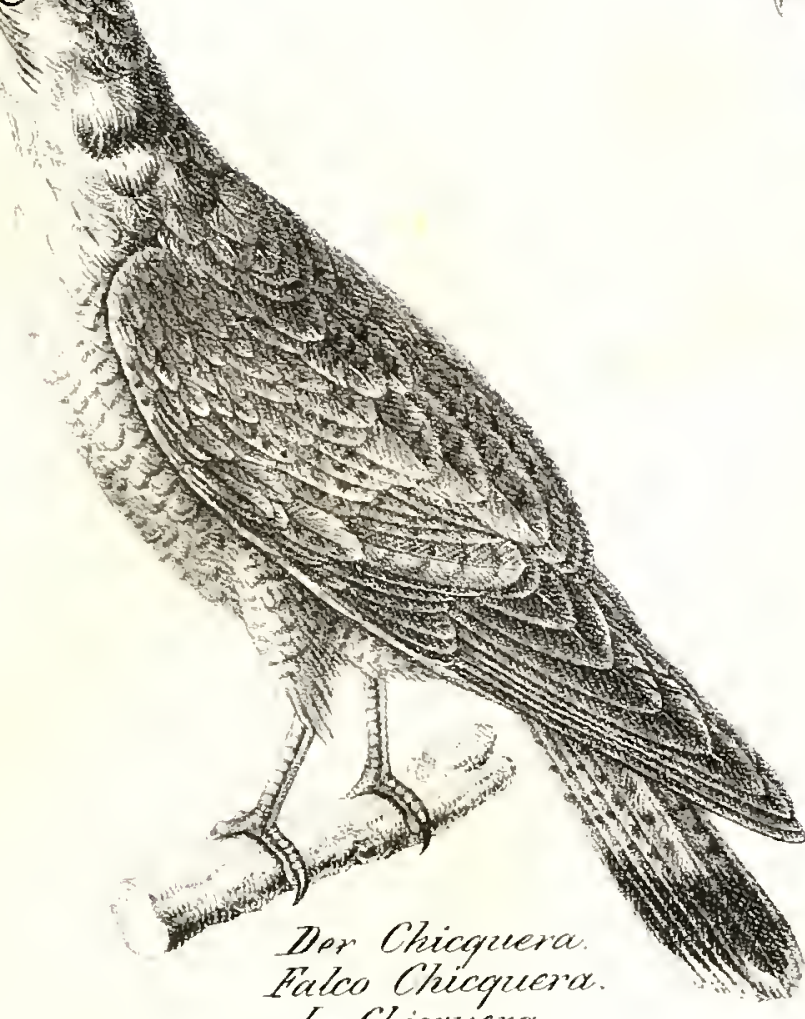
lo Chicquera.

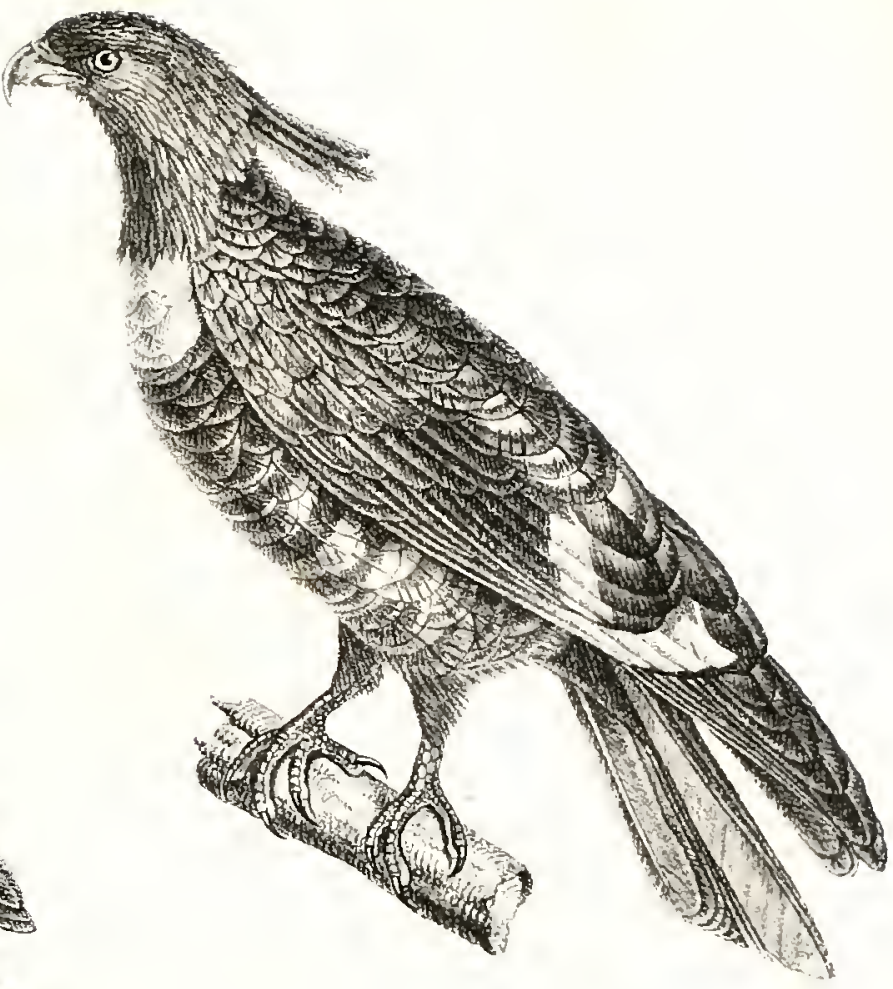

Getumbter Talke

Falco lophotes.

Faseore hespport.

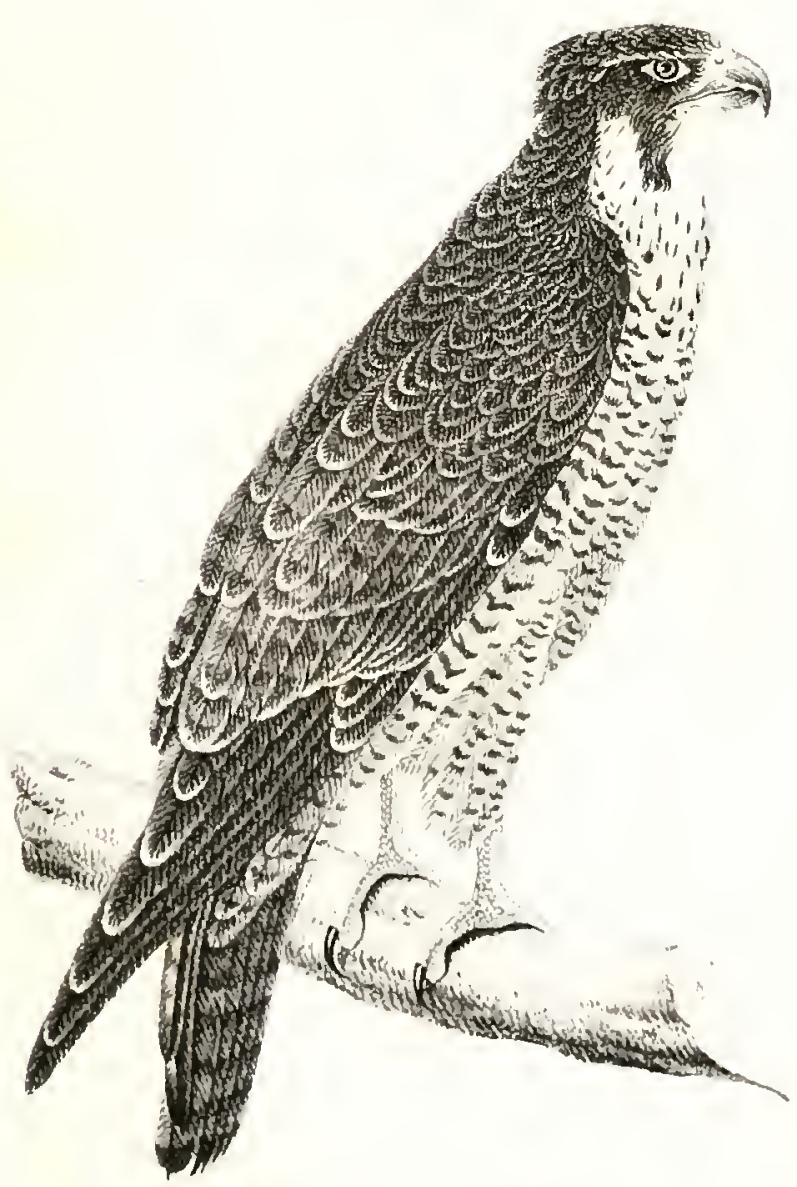

Dep Truberfouthe.

Foulon perequinum

finueson pietorin

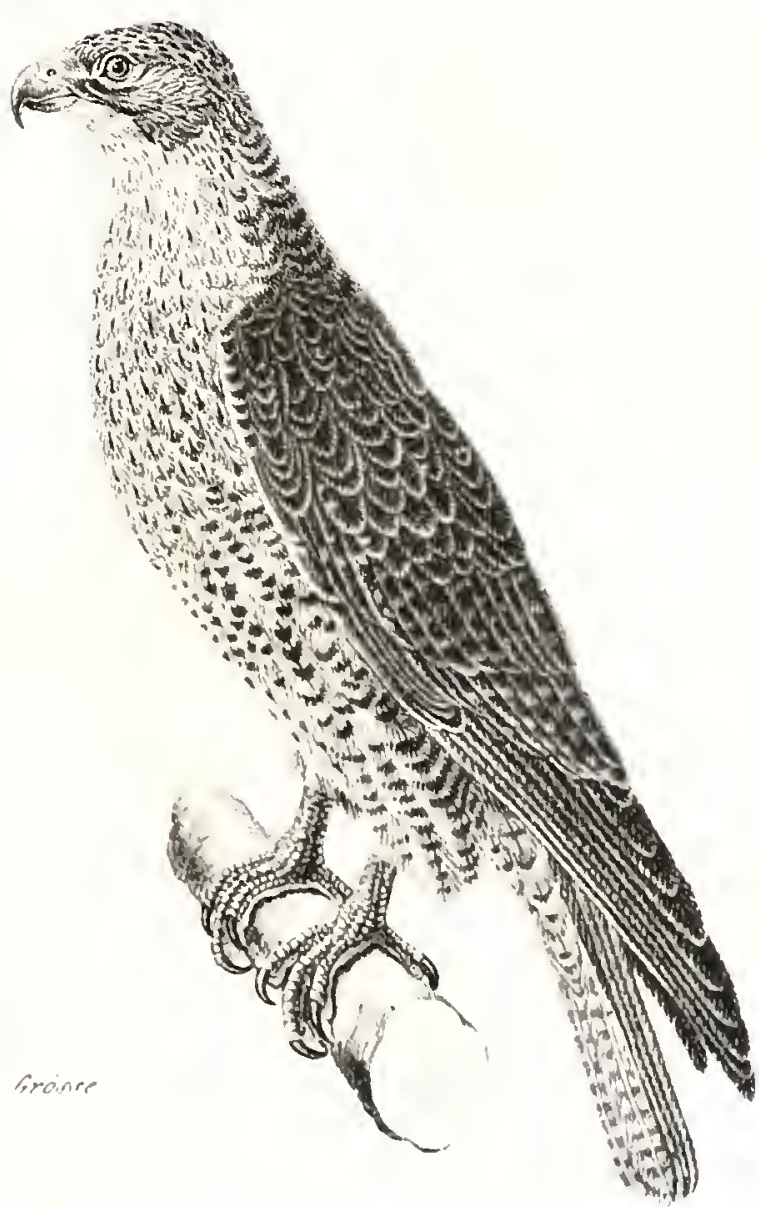

Der Geienfultit

Frateo istrondirner

Freron Gerfout 



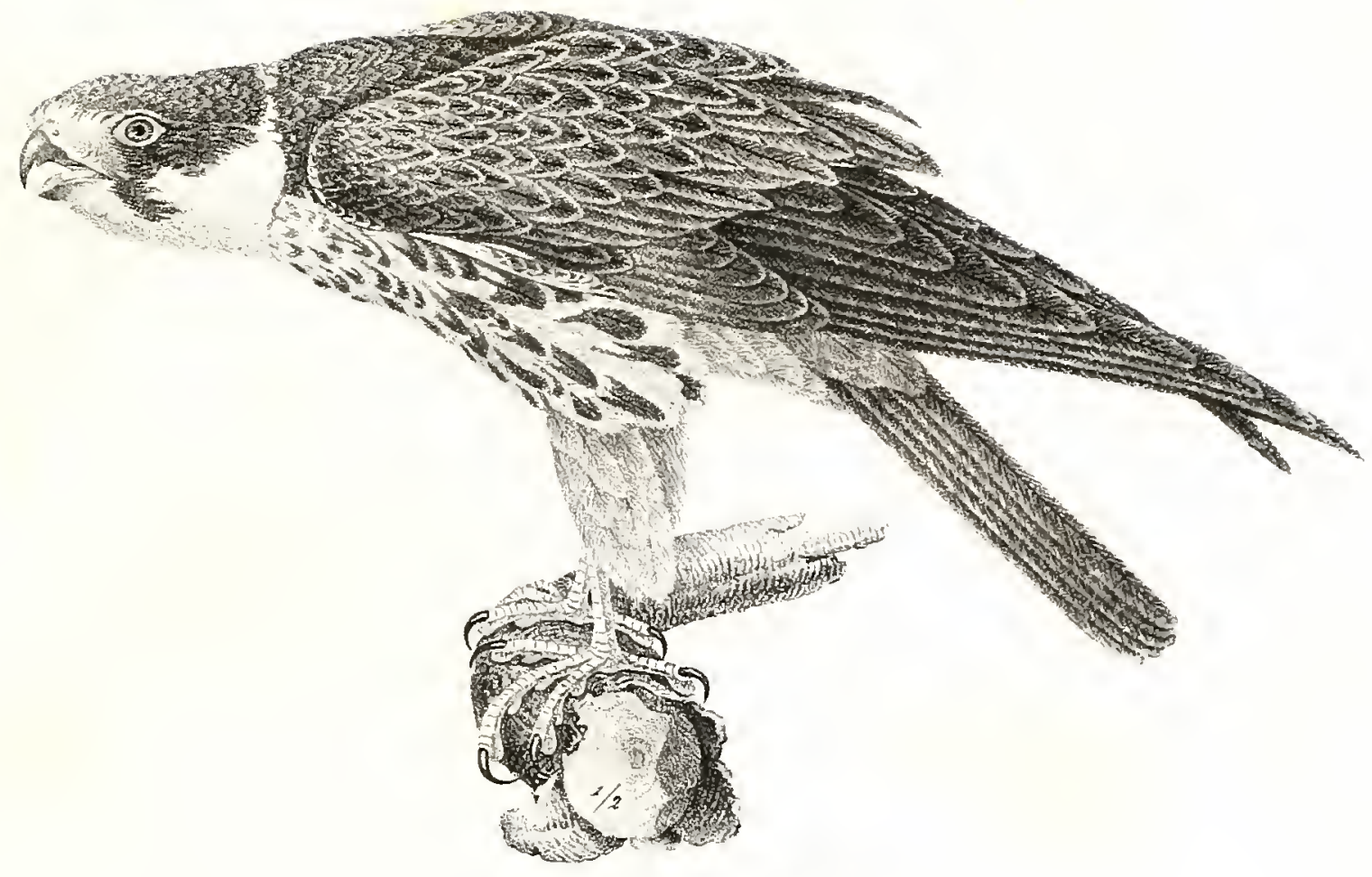

Der Lexchexifalke. Baunufaltie.

Fruleo mubbuteo.

faucan hobercu.

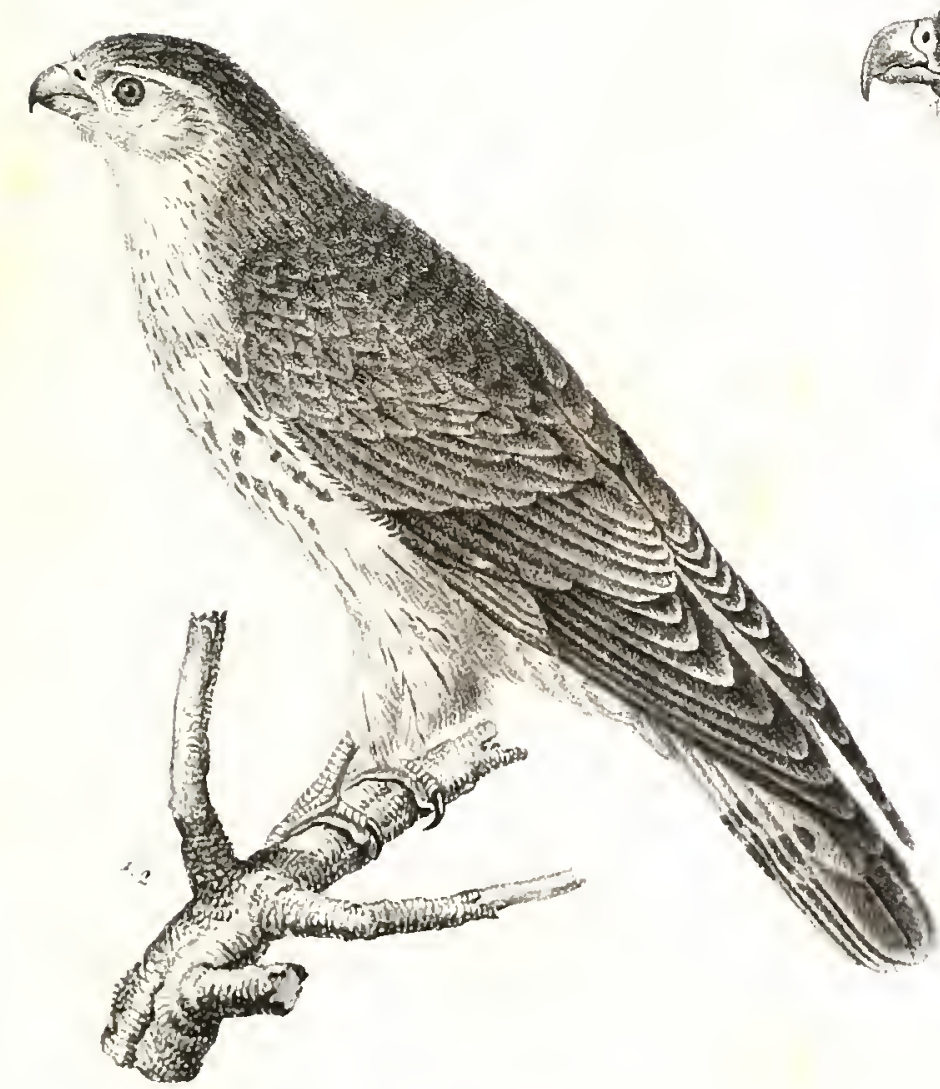

Der Merlisefultir, Plenefirthe.

liateo rescator.

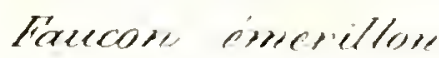
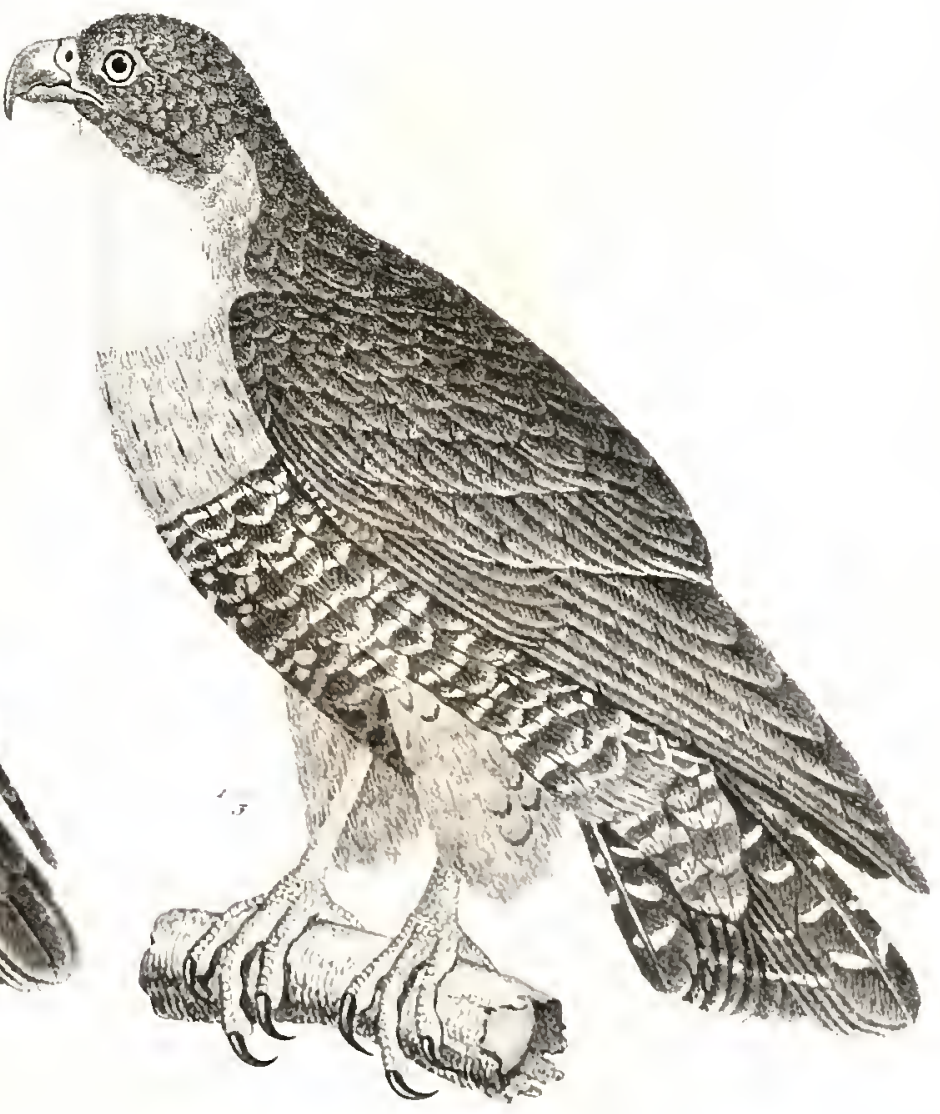

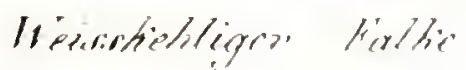

firten alboguetron.

fermene ix genege blexuster 

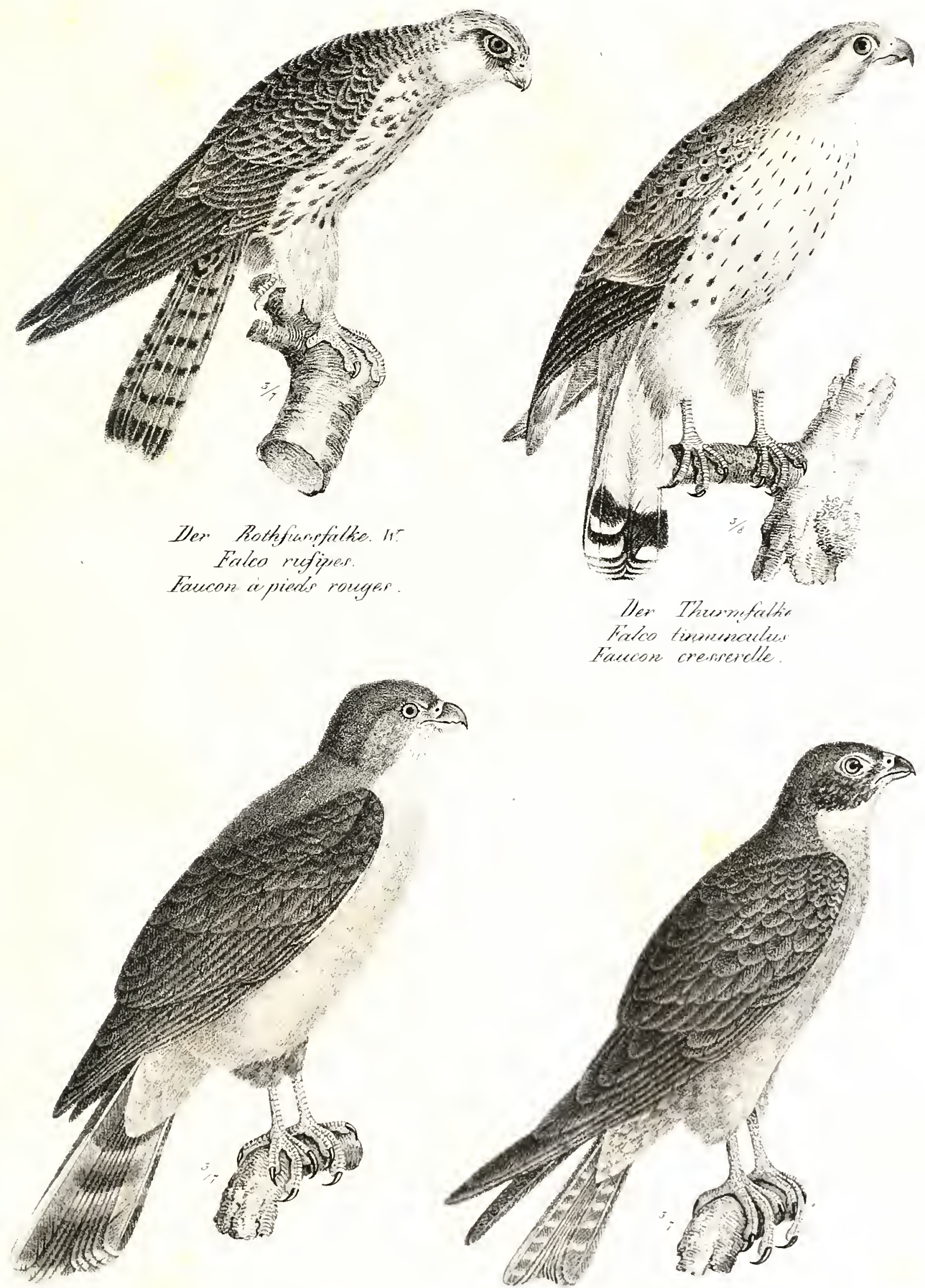
Eneizrhniger Fraltis.
frilon disdor.

Fuecon diston.

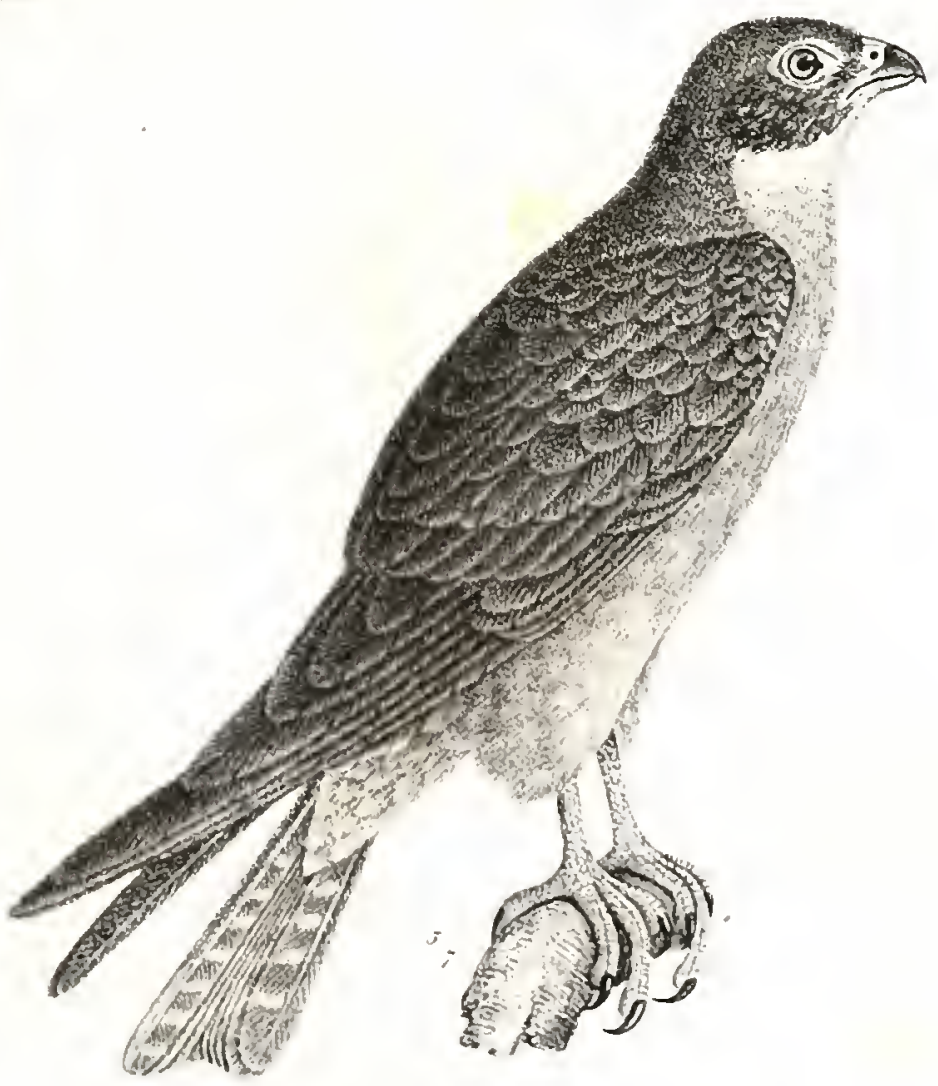

- Arteovandiricher Fathe

Freloso Aldeorasudene

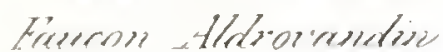




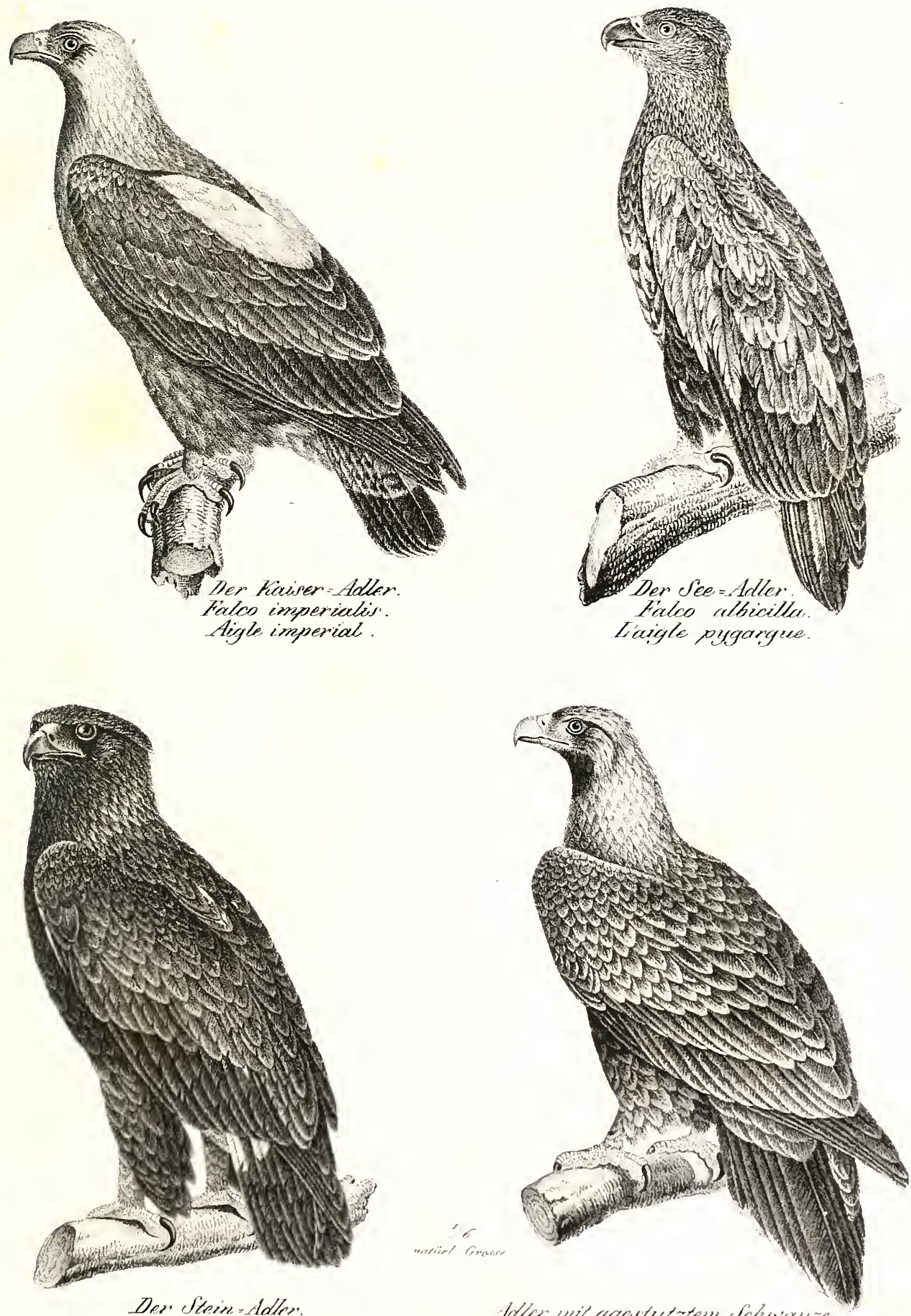

Der Stein-Ades
Falco fulver.
Aigle commun. 


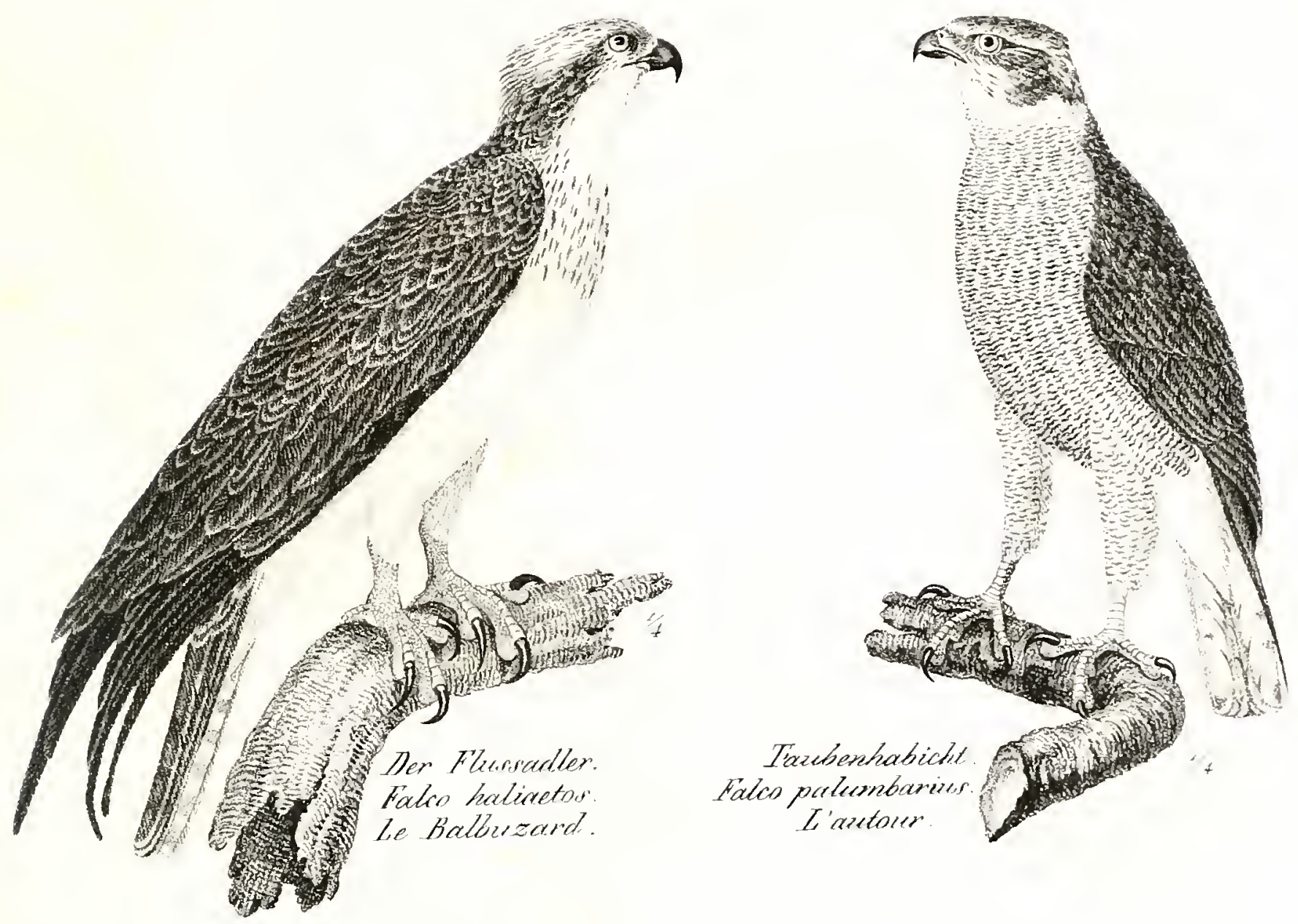

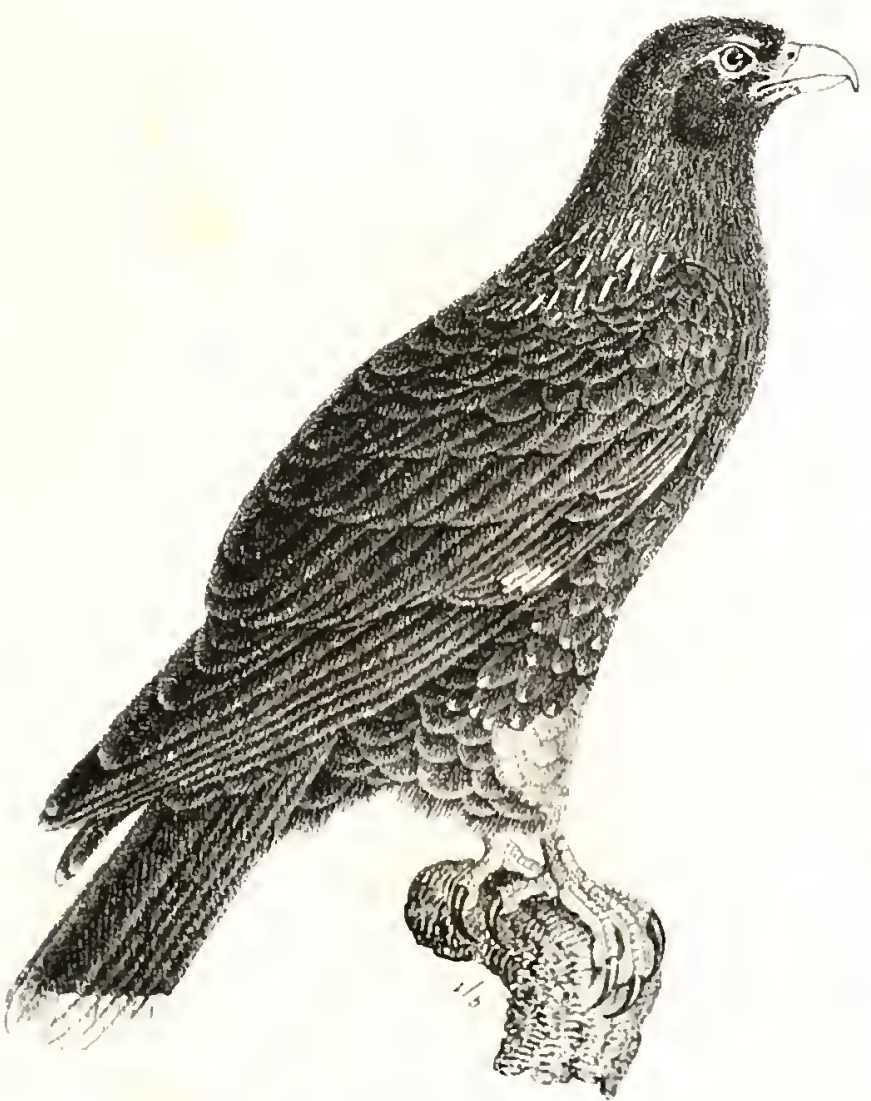

Der Coursureser

Falen novere Iclasutime.

Carresera, fissiber

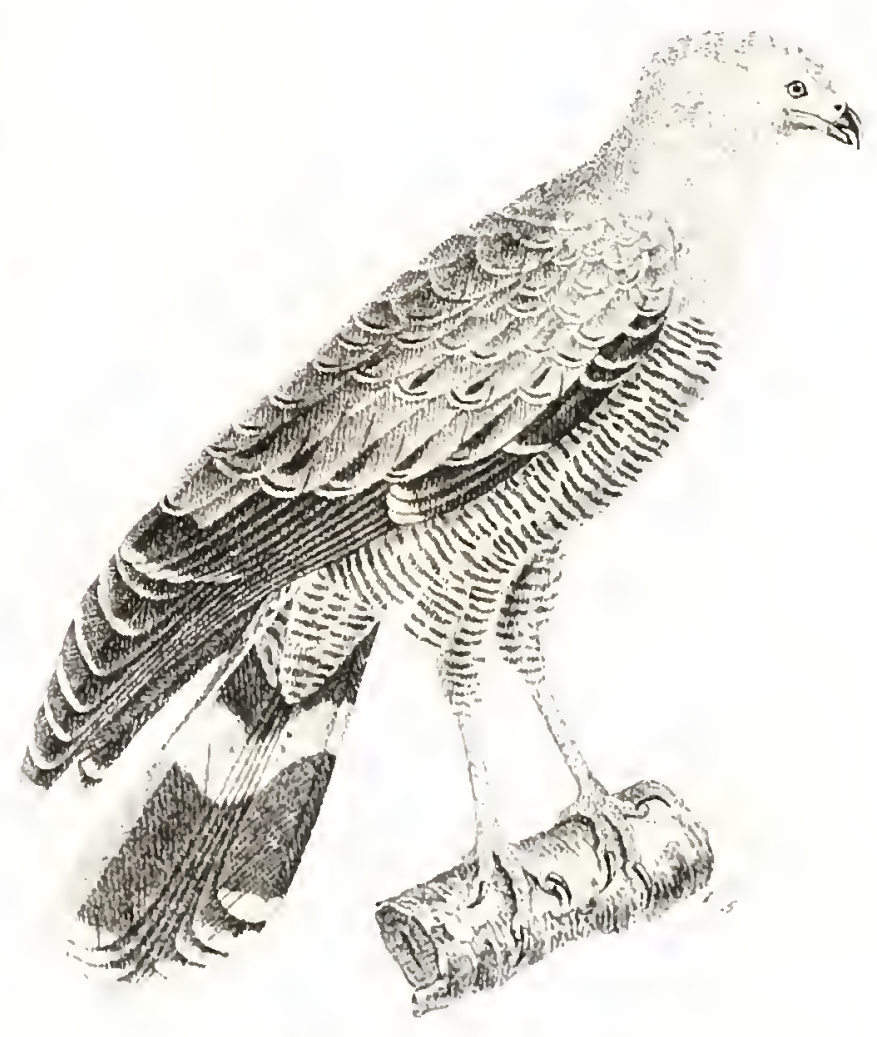

- Vastitherstieges Habicht.

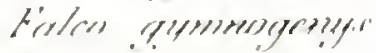

Alesmes gorese meses 



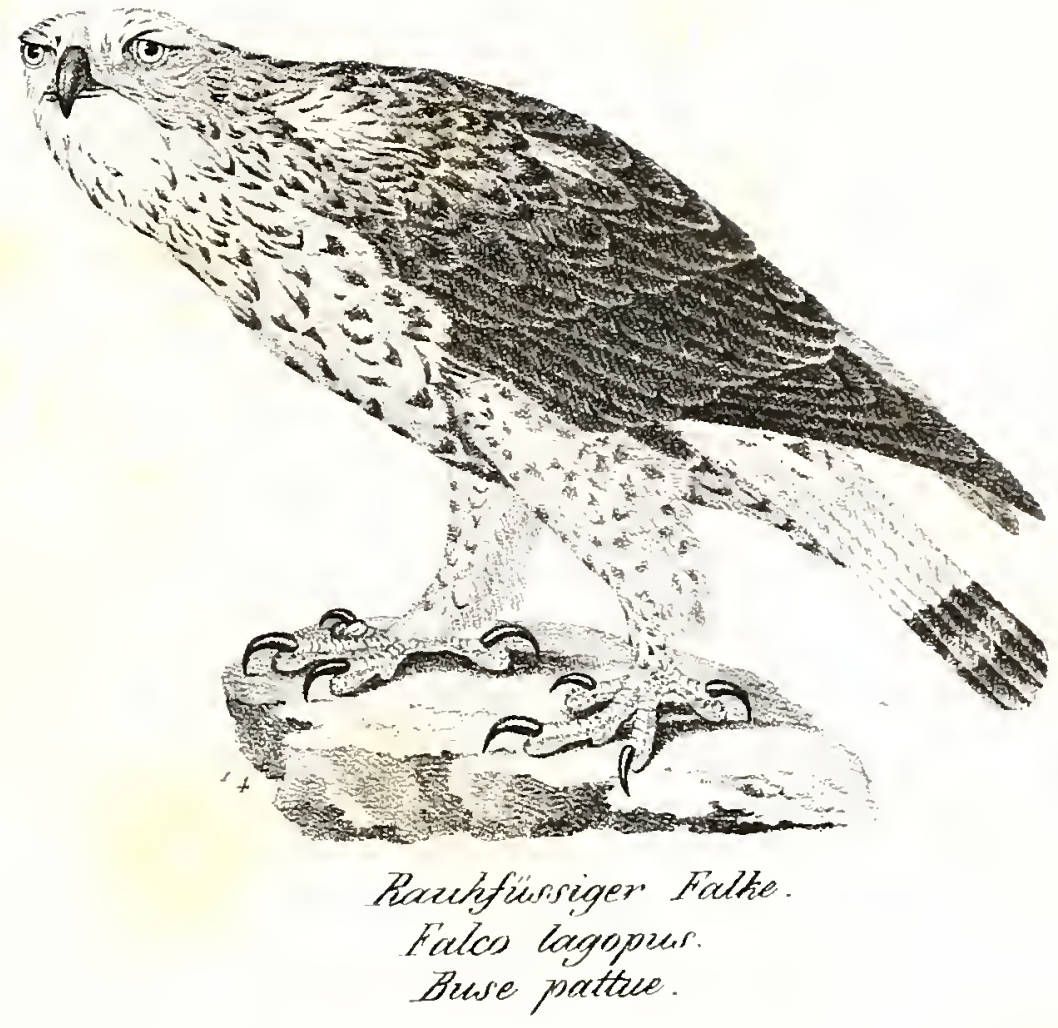

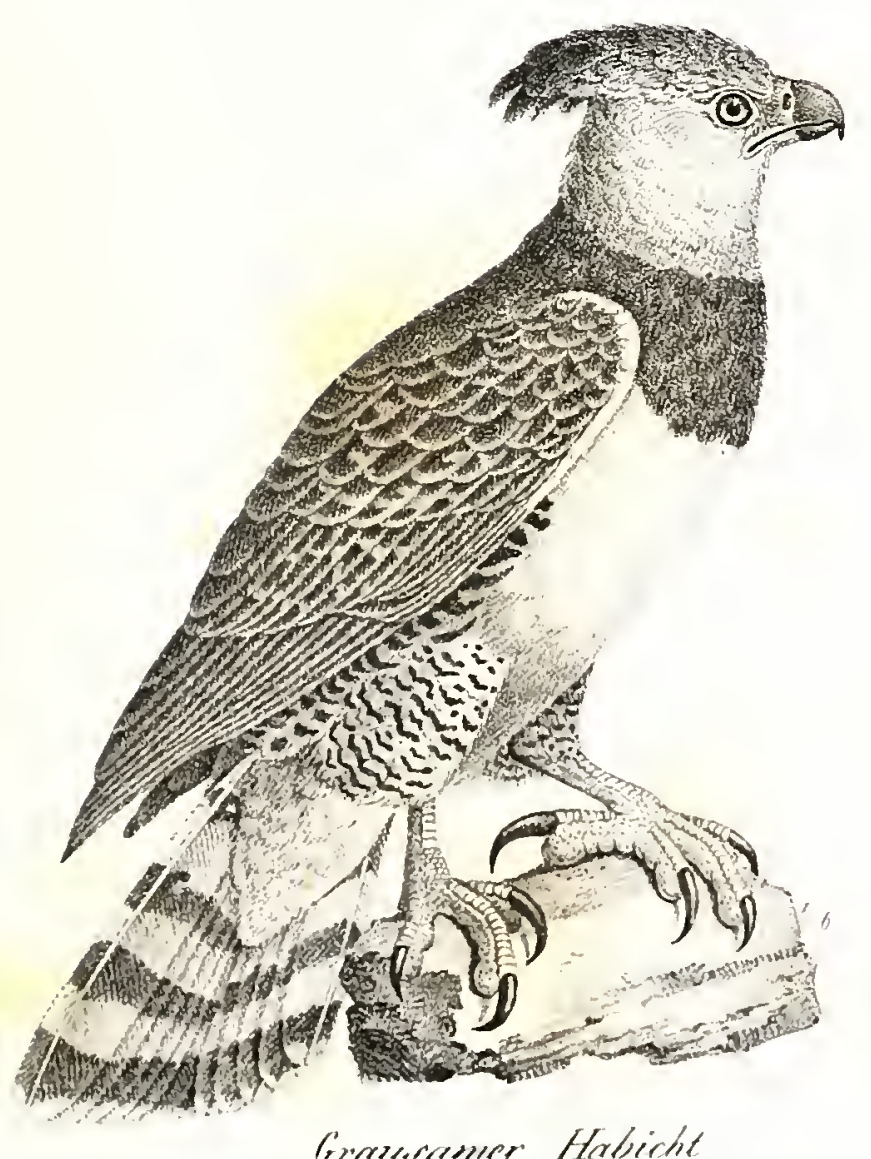

Gremerremer Hobicht

Fales dertuelnr.

Lutour dristouctors.

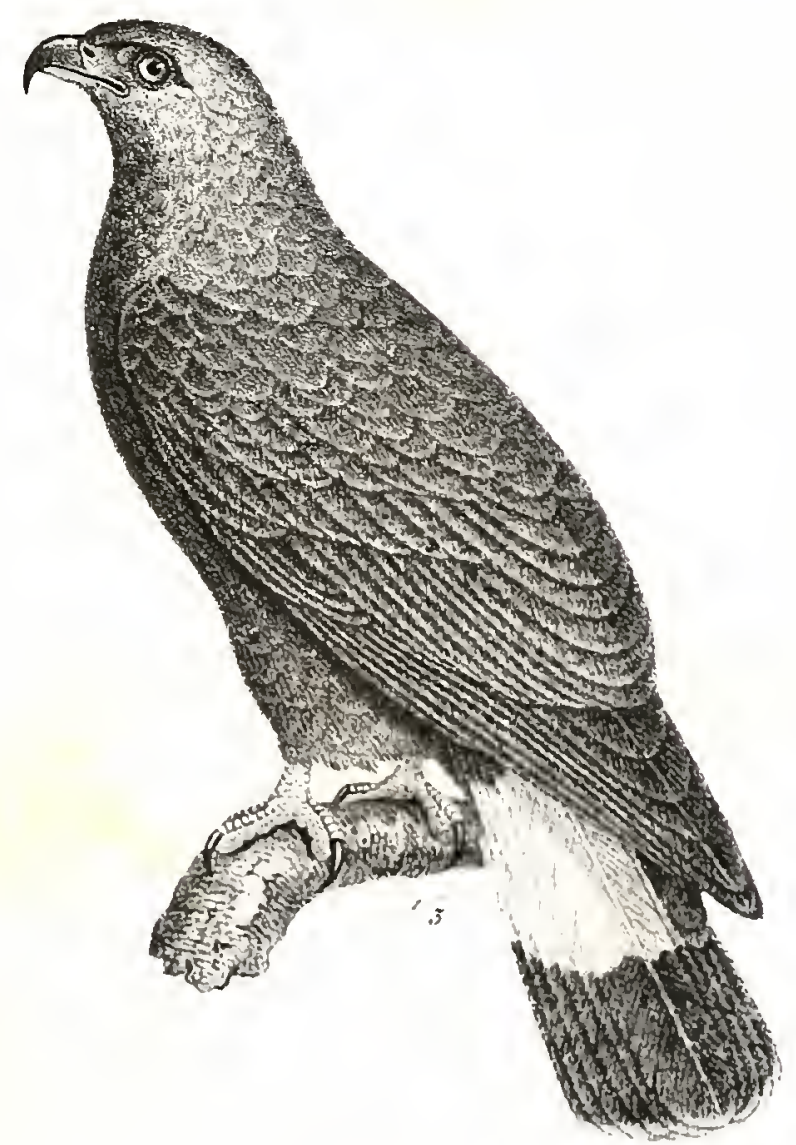

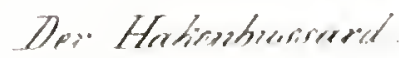

finten uscinatien.

Cumender bee en cror 

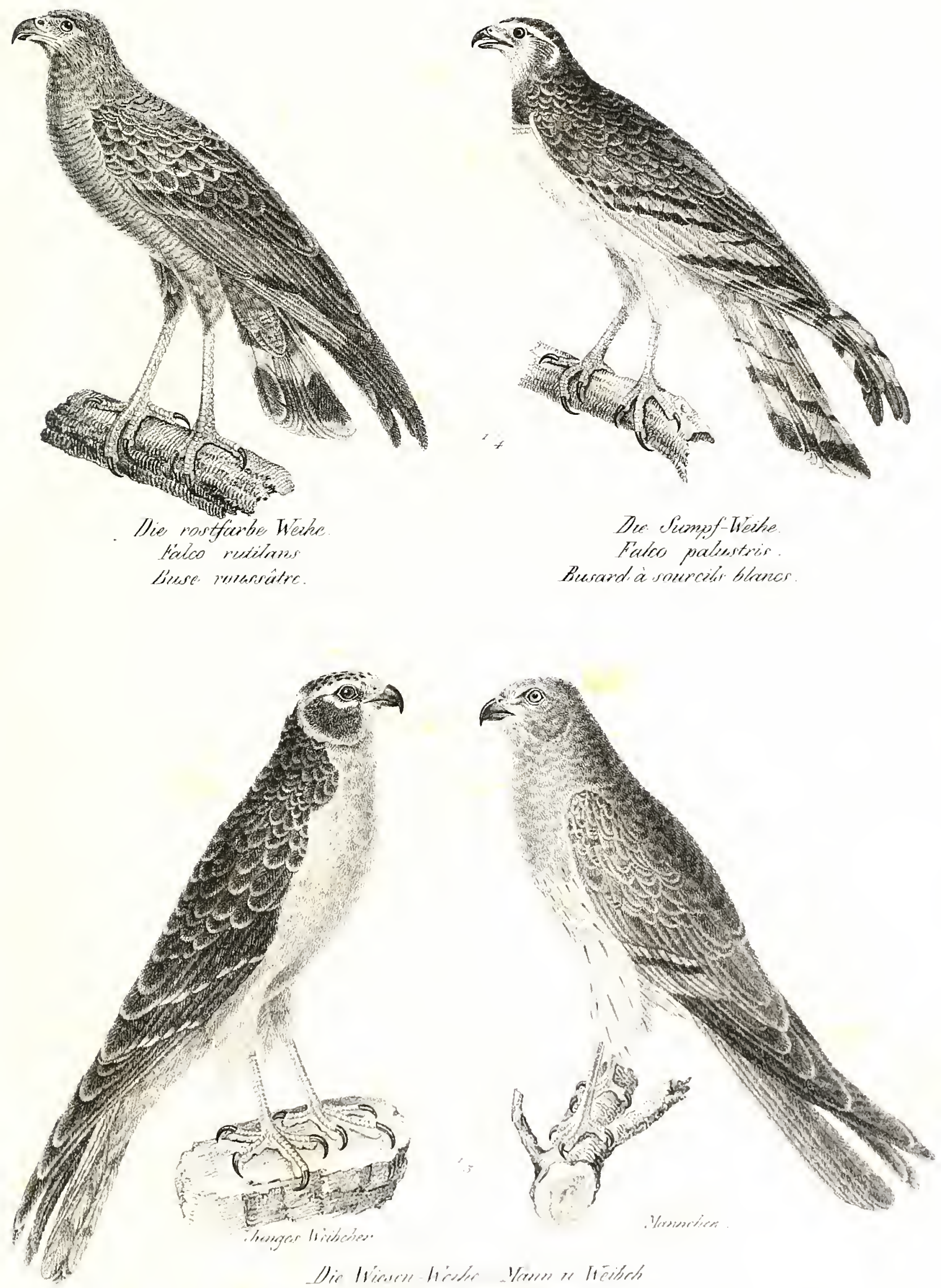

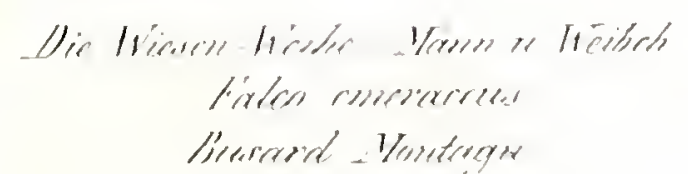




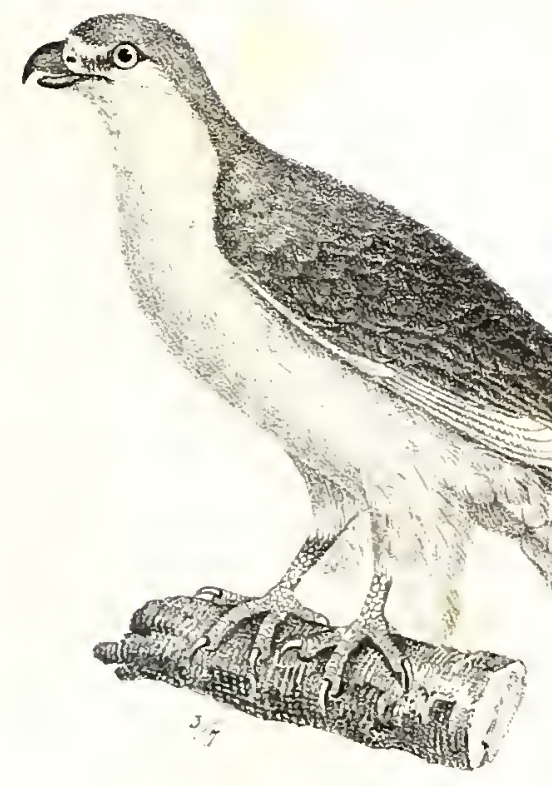

Hiteiner Hilan.

Falco Riscaur

Milex Riocout
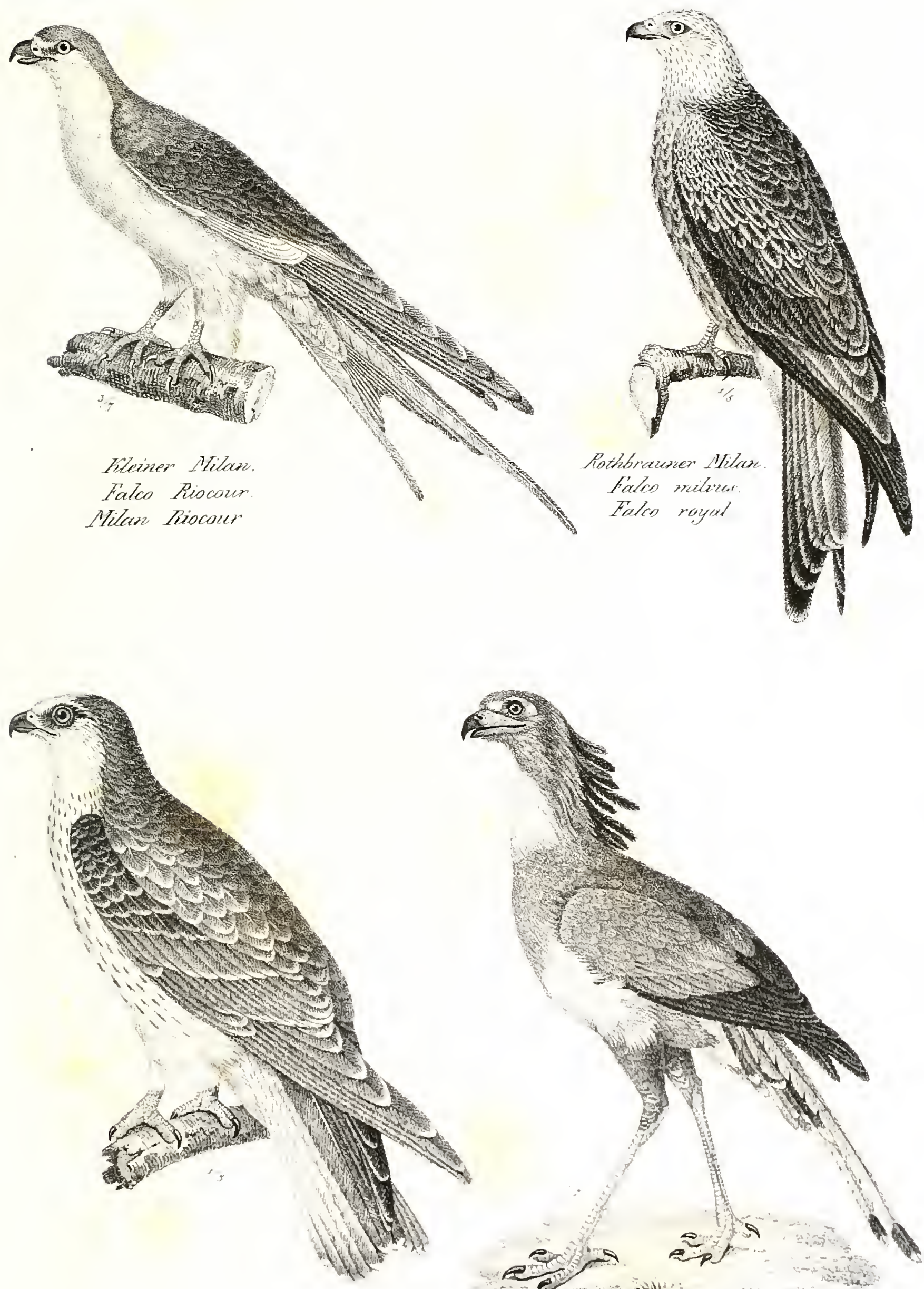

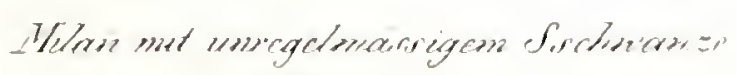
Fatro dispas

Milas is quese irregulise

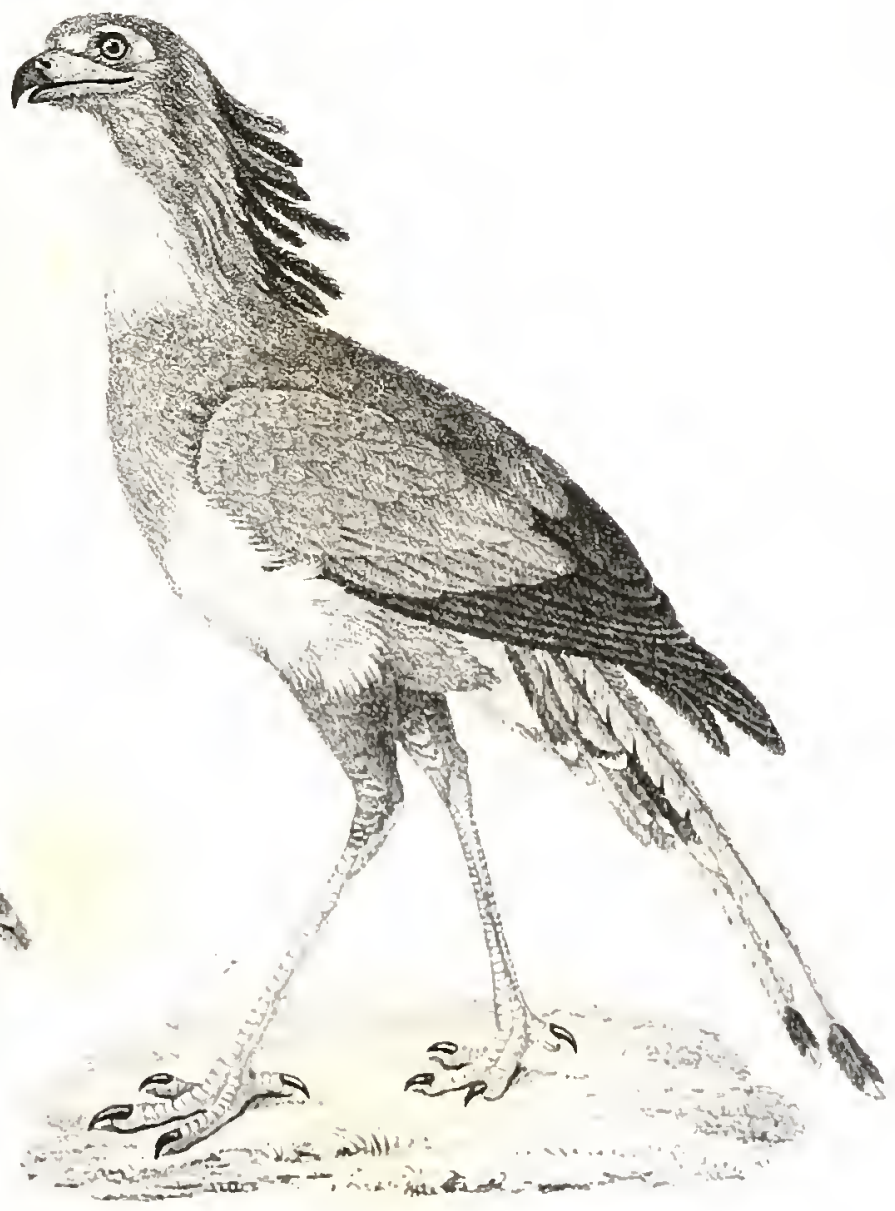

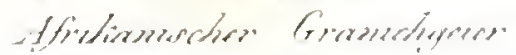

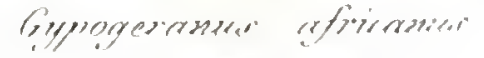

lis Merwager. 

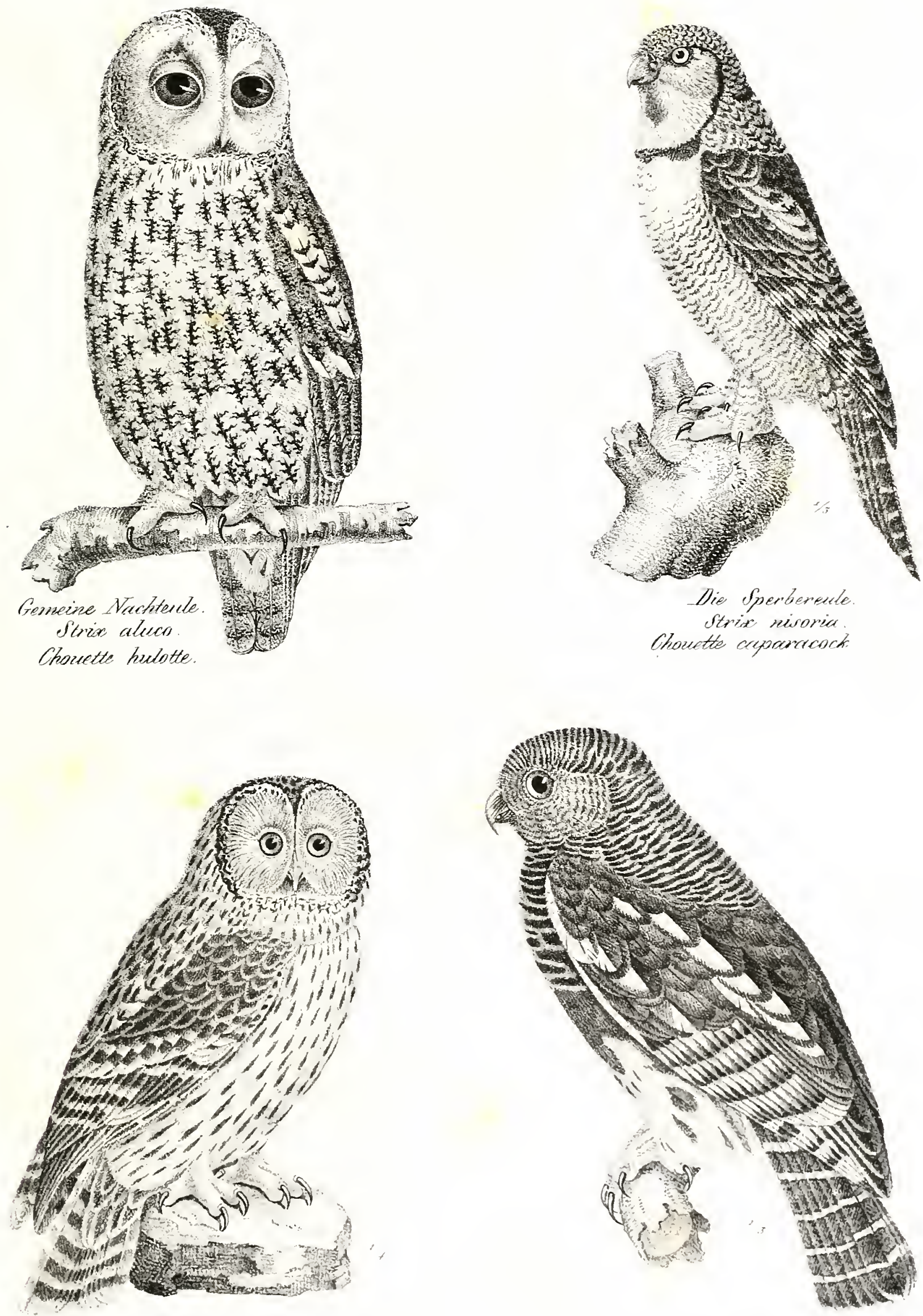

Dir Habichtersule.

Strix userteruris

- Chouette de l Ourrel 

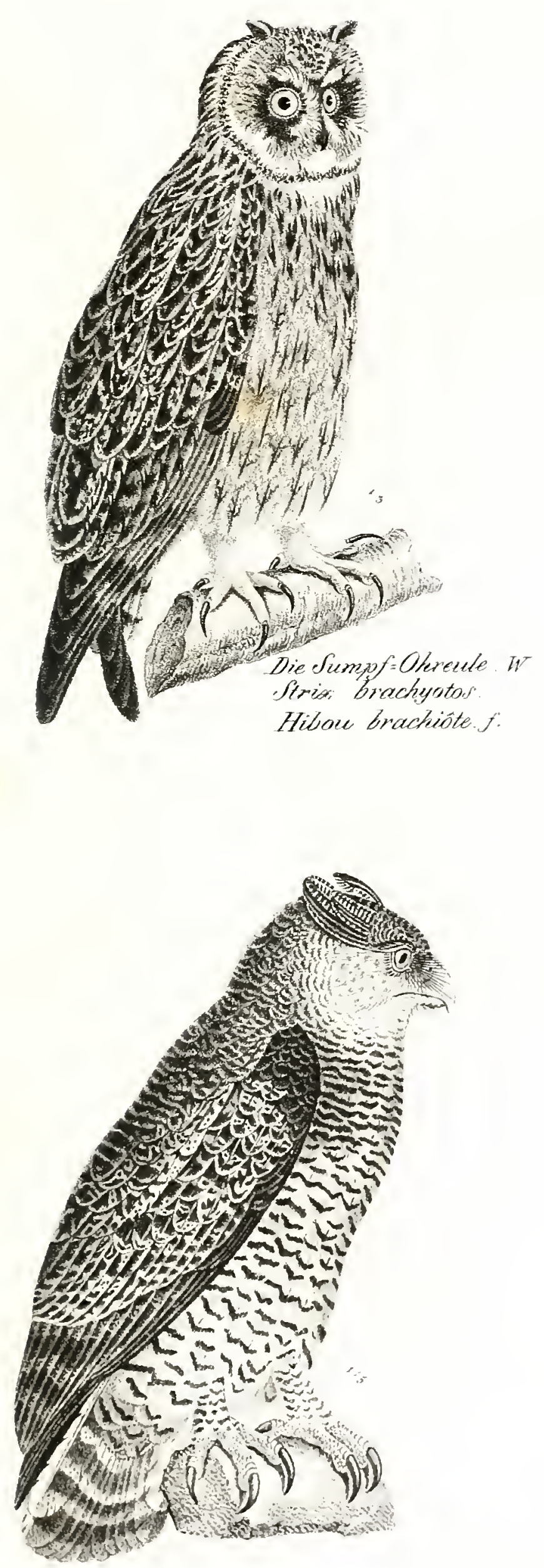

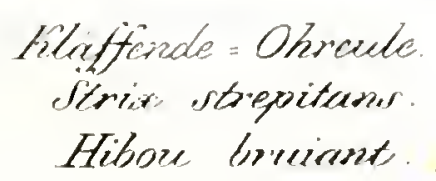
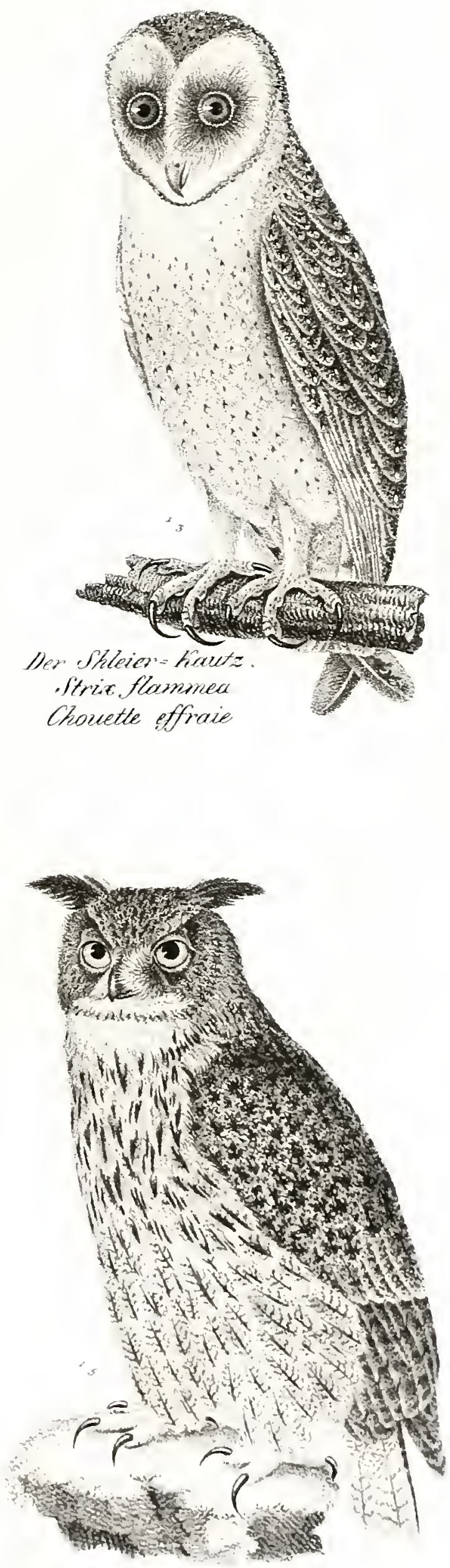

Dir Uhere = Ohecents. stoit bubo

Hibou grend duc 


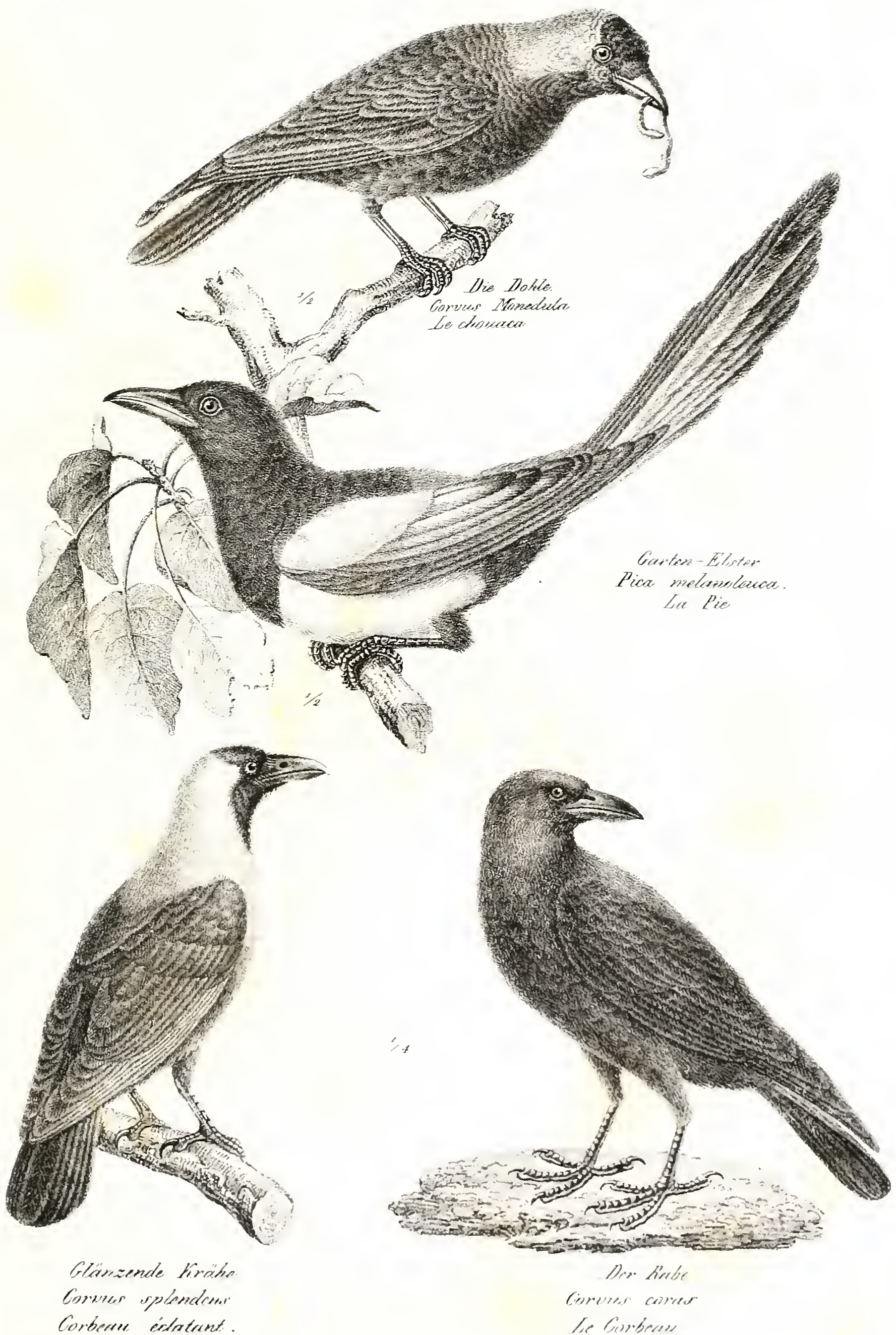



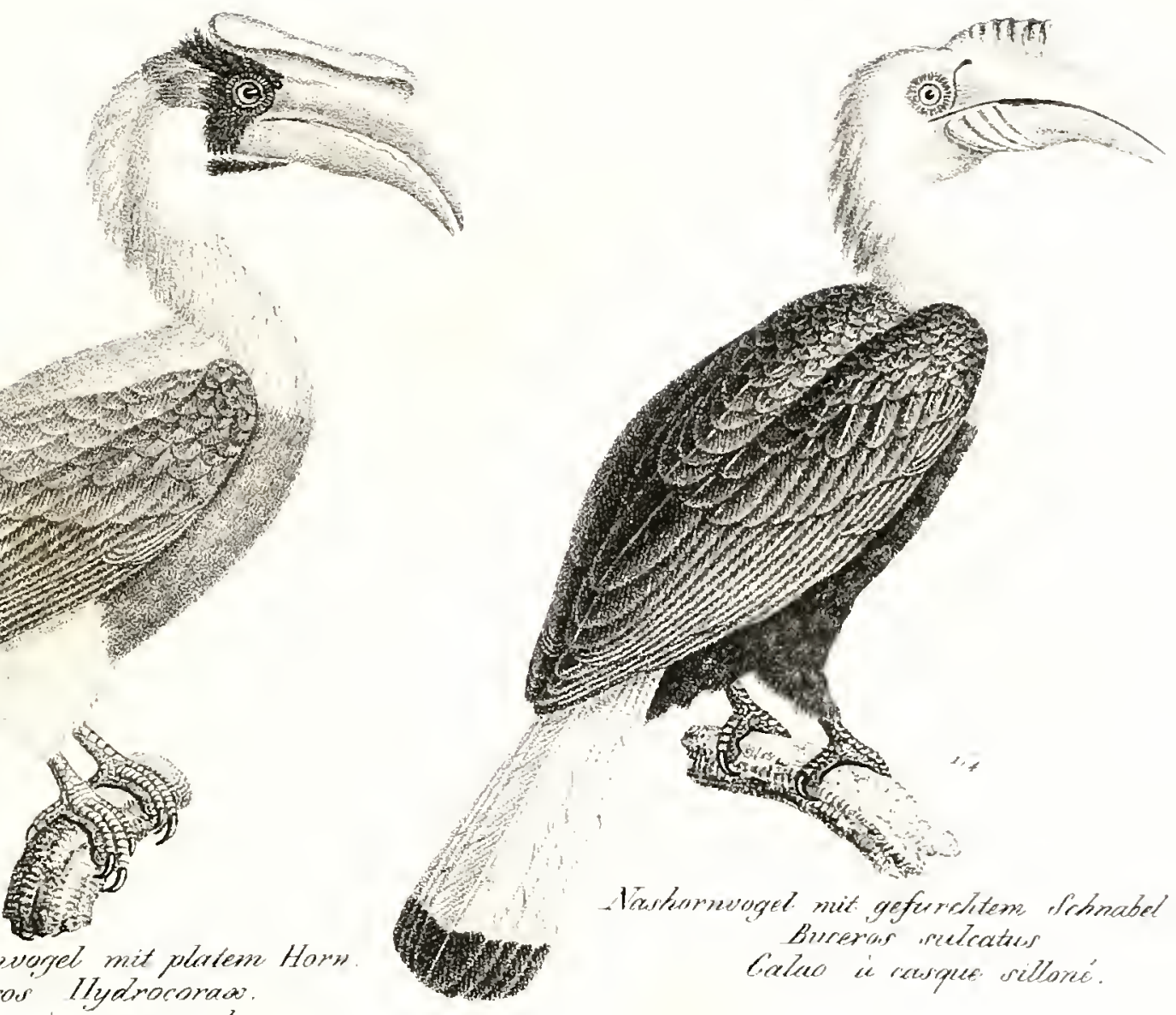

Nashompurgel mit pletem Hown Luereras Thamoconaw. Calan a caspree plat.

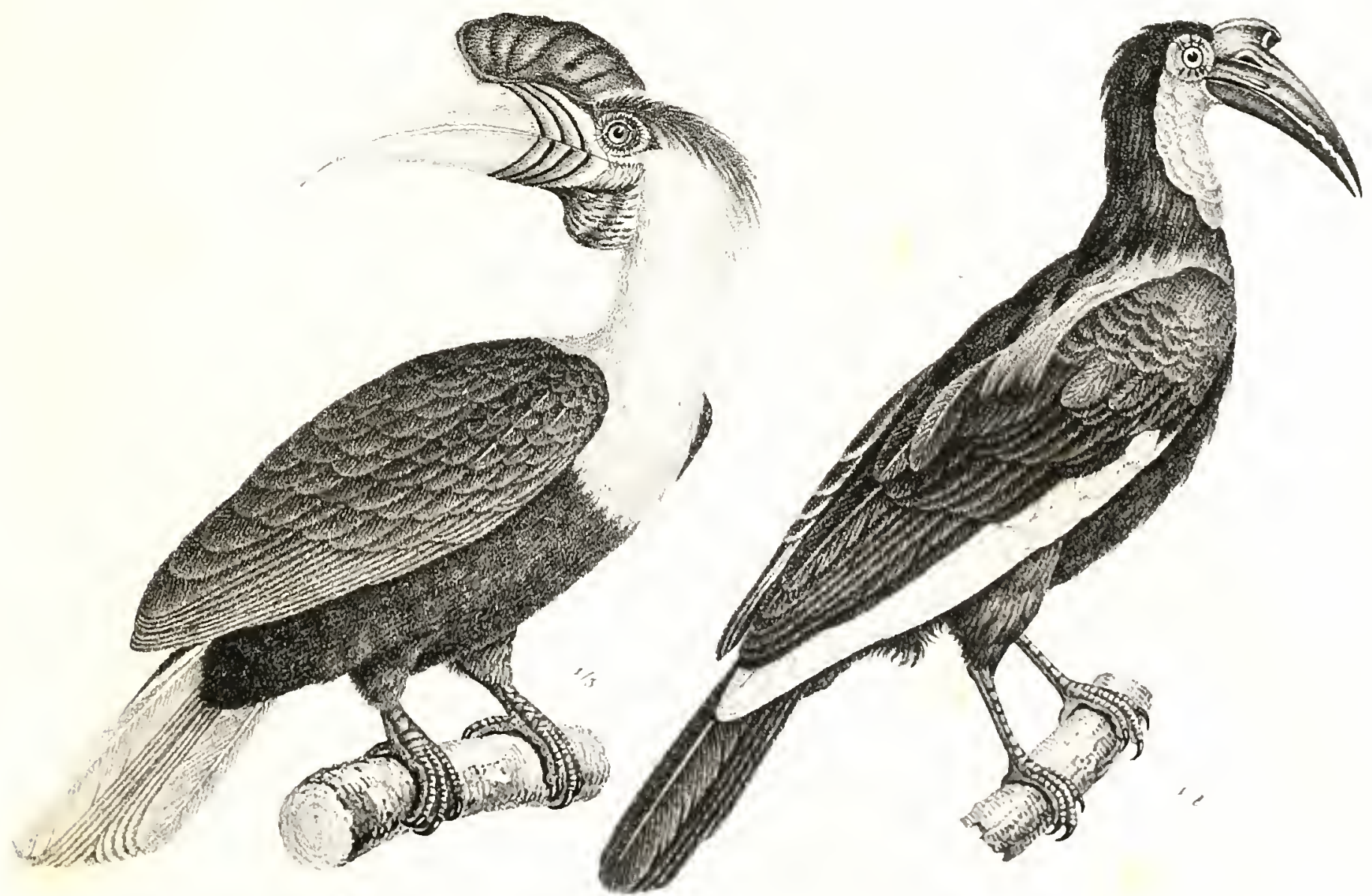

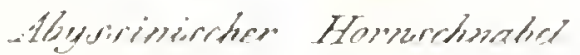

Buecerar abyersineses

eratere crevenecule 

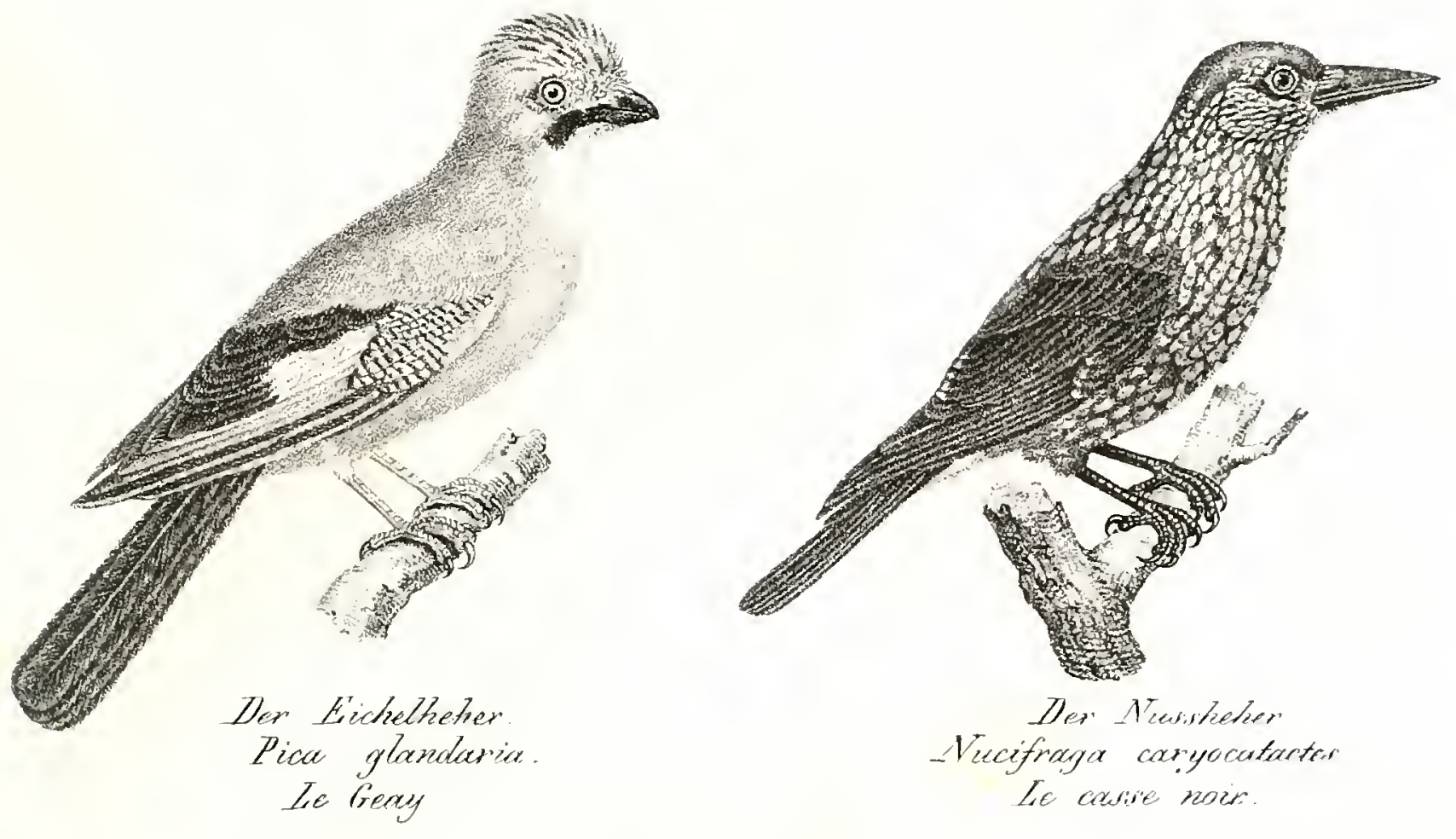

- Vecifraga coryocatarter

the covive nois.

$1 / 3$ der natül larcuise

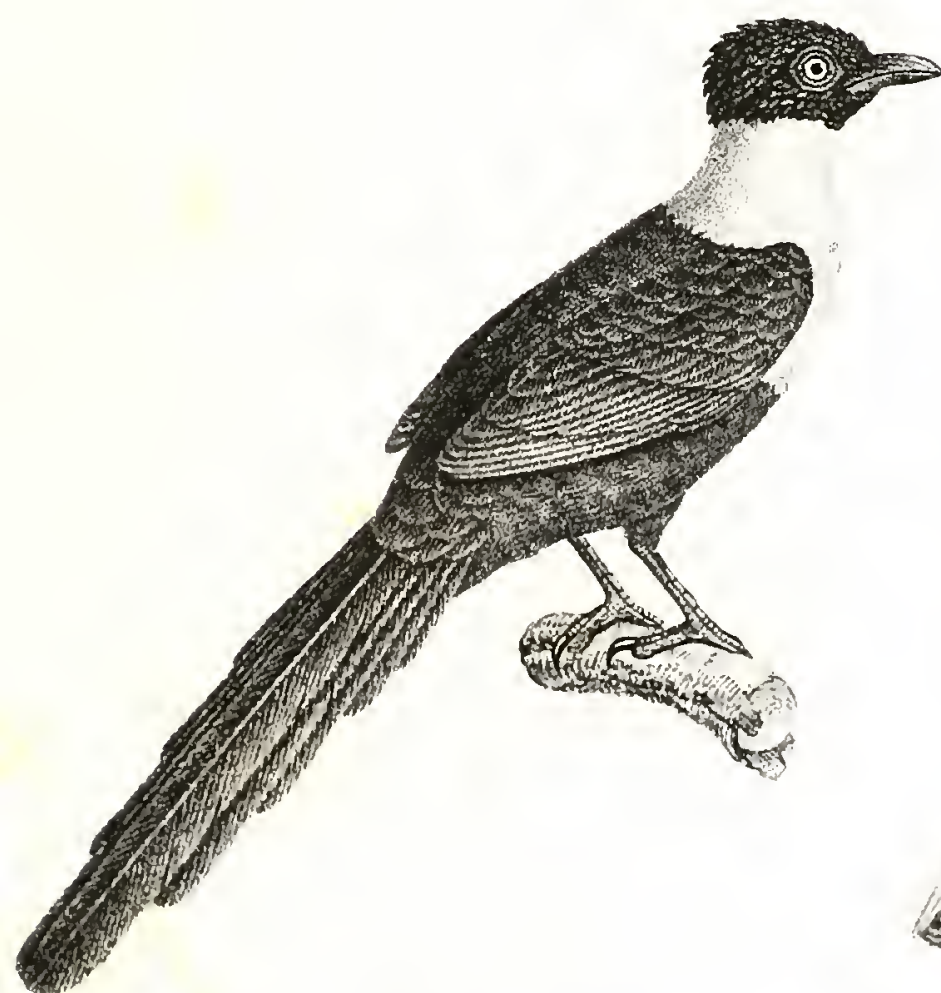

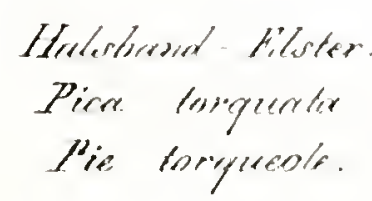

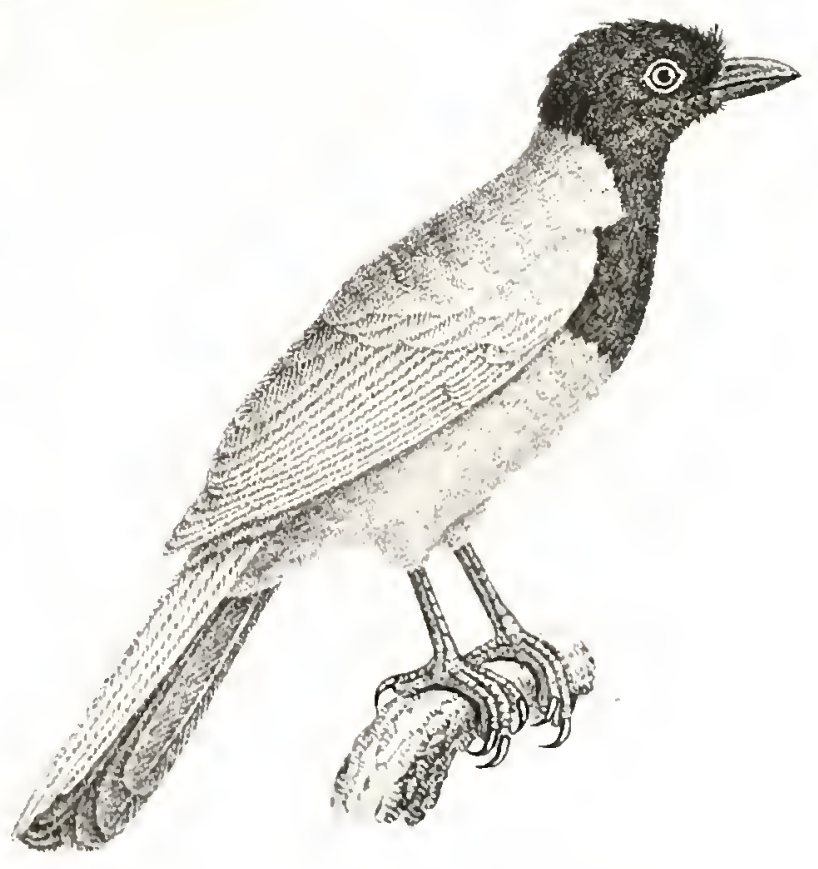

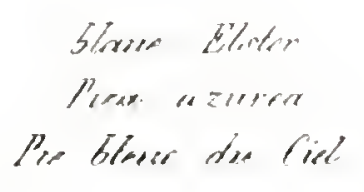





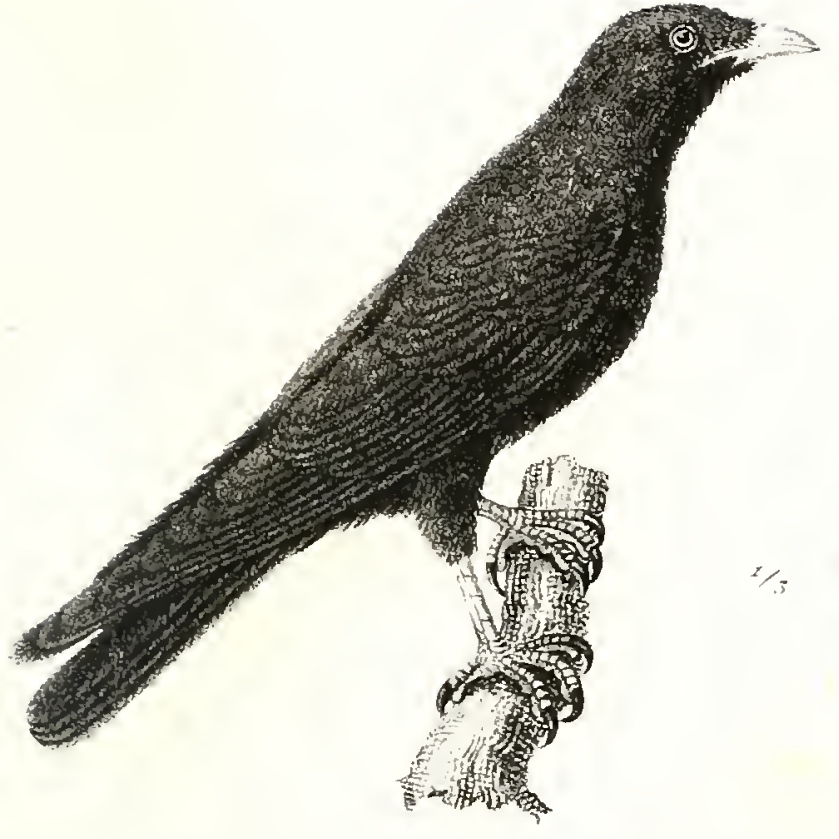

Gelbsrhuceblige Drosseldotule.

Pyrrhocorax pyrrhocorcex. Lie chenquard.
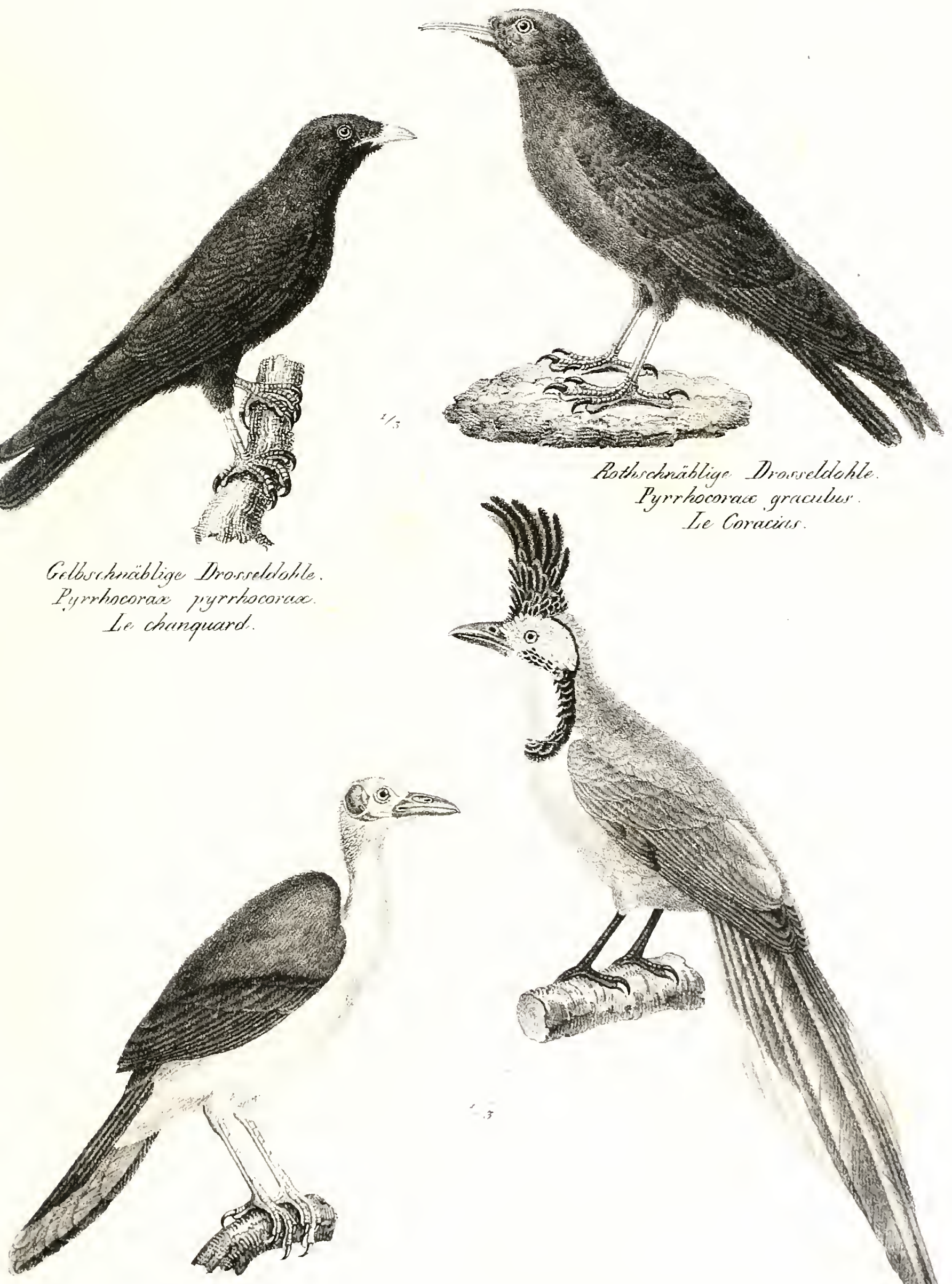

$$
\begin{aligned}
& \text { - Wretithiopfinge Elirter } \\
& \text { Pirn gymensecphalds } \\
& \text { Pie chroule }
\end{aligned}
$$$$
\text { Gederesbie Eilosten }
$$ 


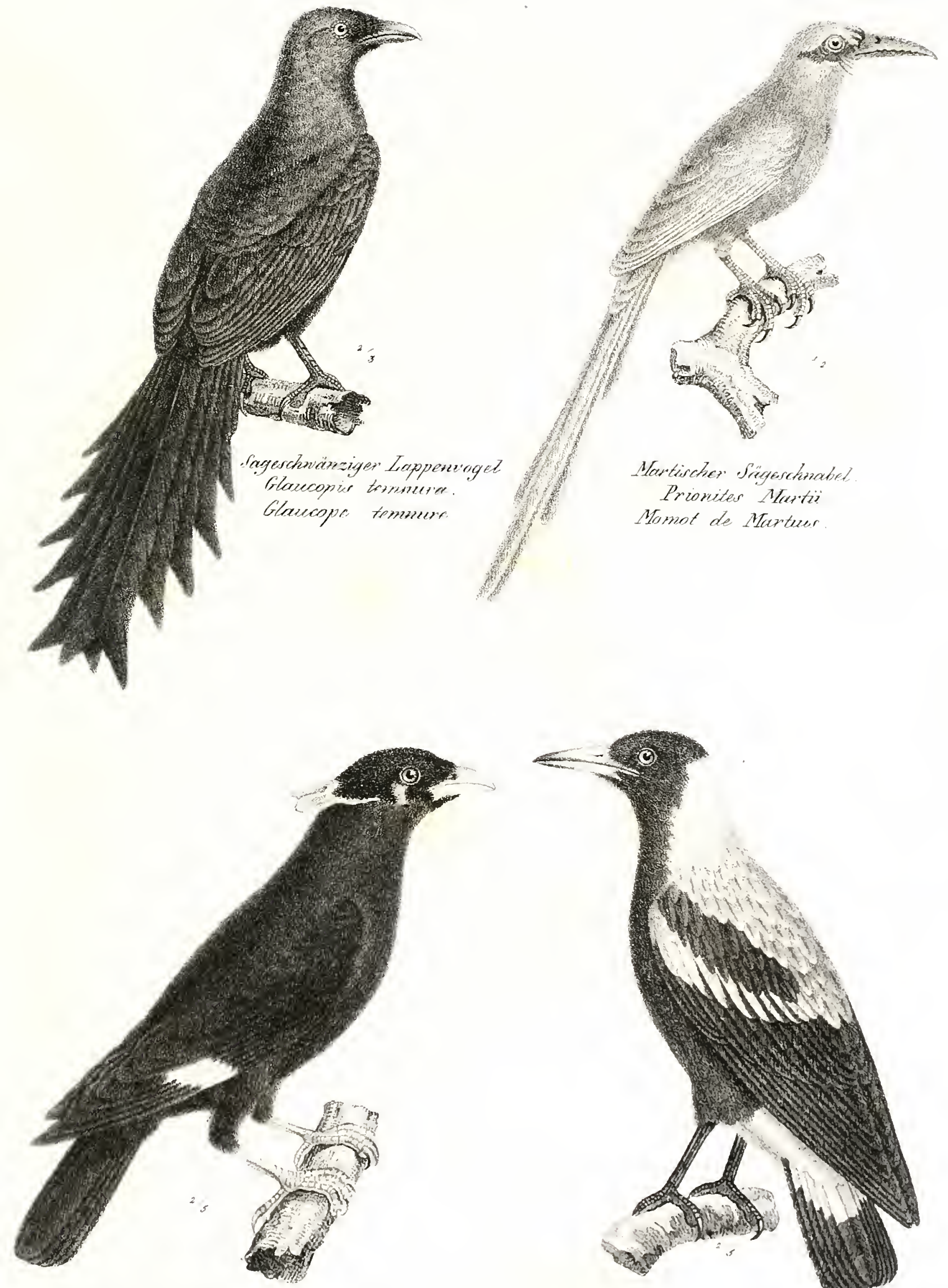

Geschnertaiger Mlen

Giorenta religinses.

I. Meresuto.

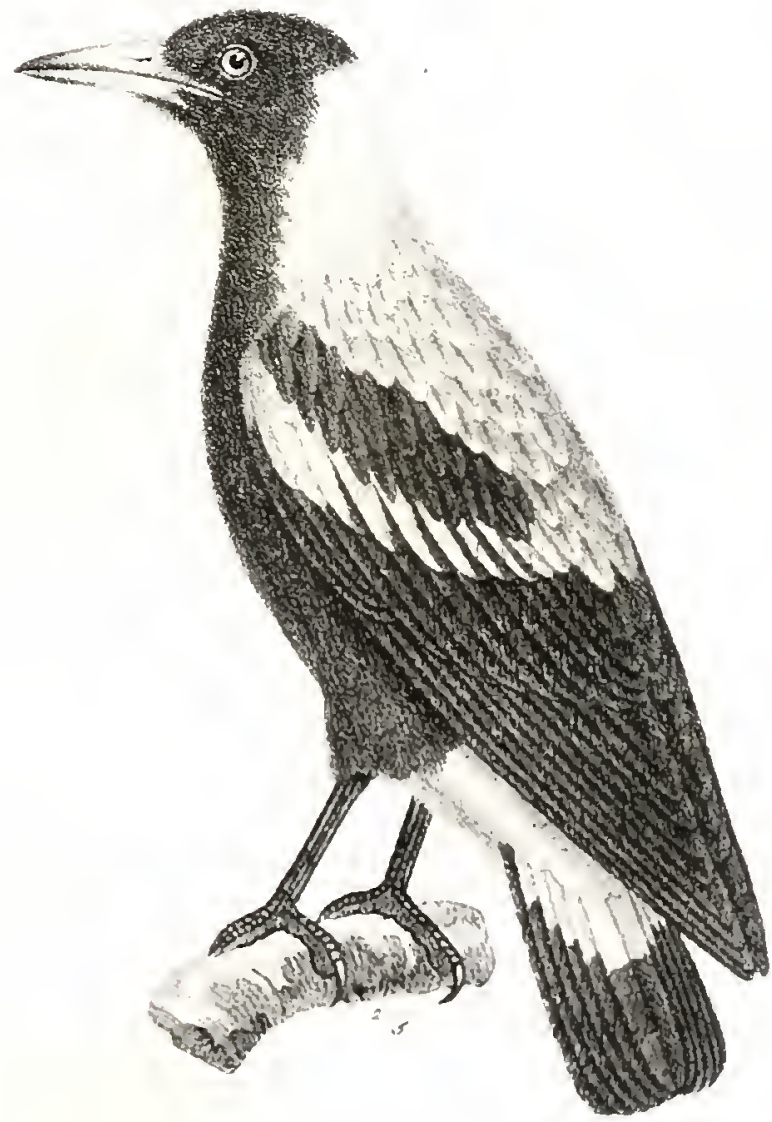

$$
\begin{aligned}
& \text { folvitende itare } \\
& \text { Bariter tebseren }
\end{aligned}
$$

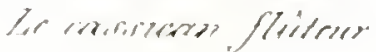





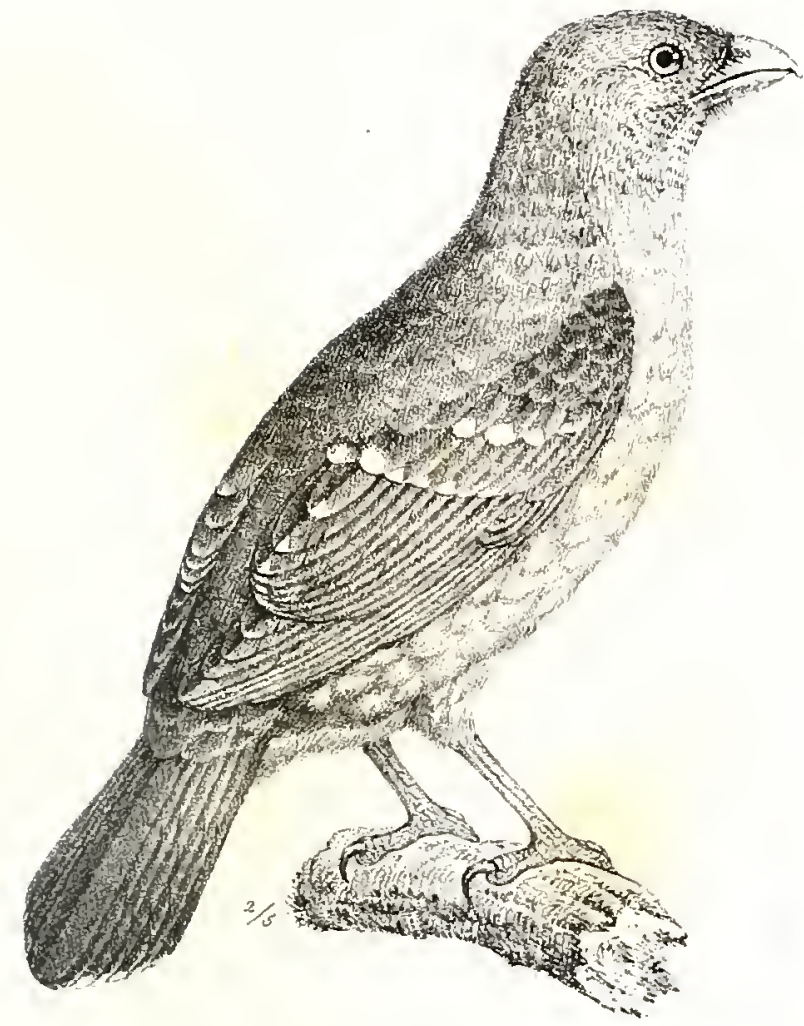

Grïnen Federrotenabel

Fistor virescesins

Piroll ecrdin

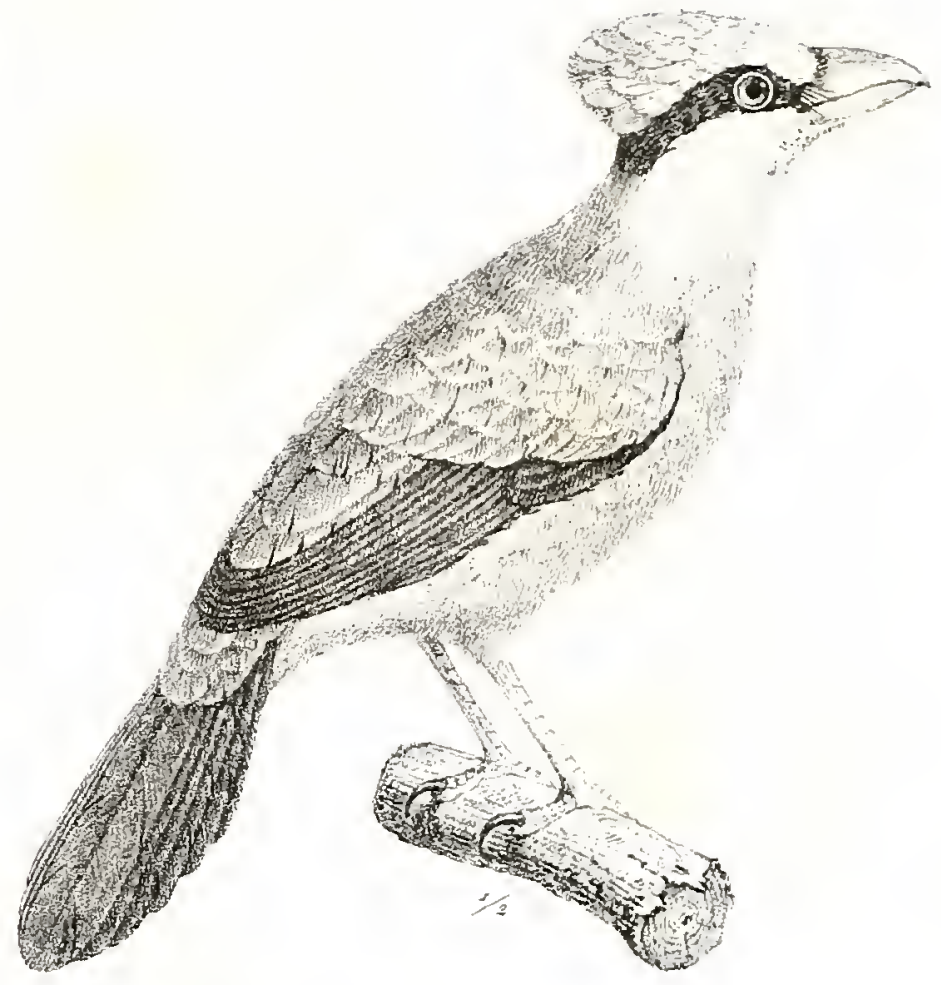

Meergrïnen Federirchunbel. fietta thatosisinse.

Piroll thatarsin

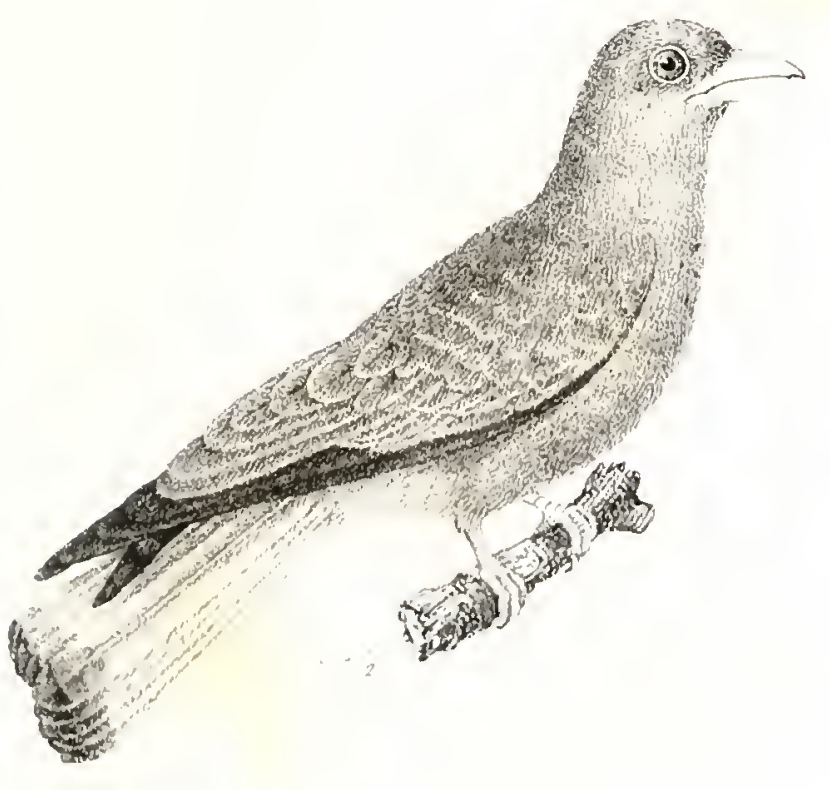

Dee Perpuervedte

Colerien nenterese

Le Piolo violet
(1)

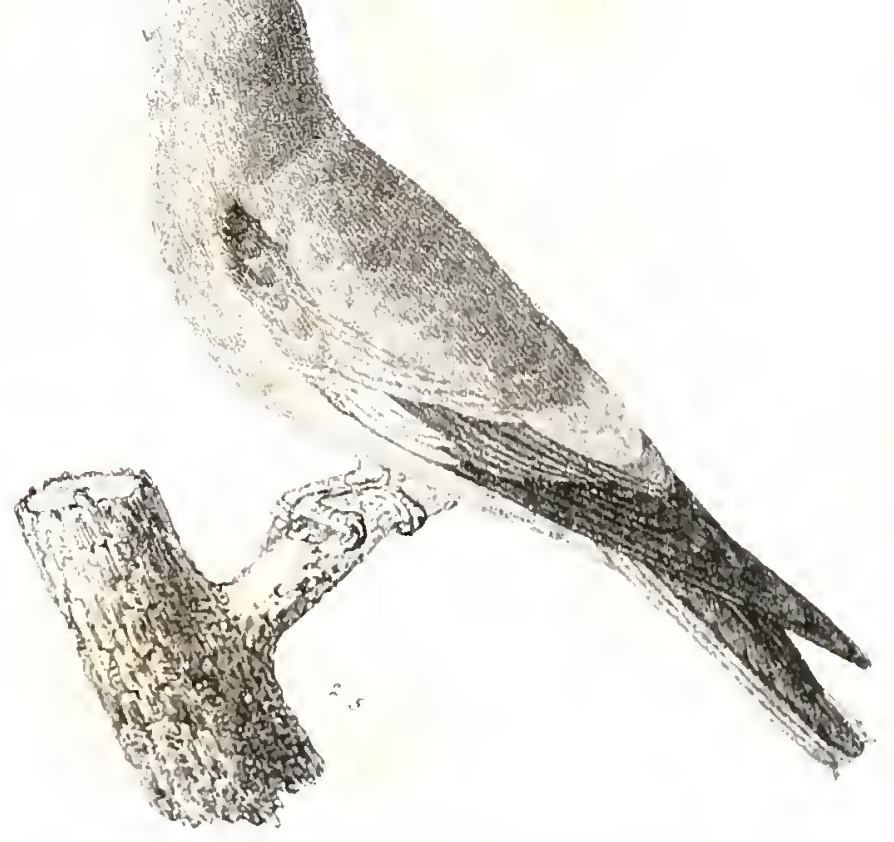

Dir Veredellisciber

Comeresen gereenters

lor fintlies. 

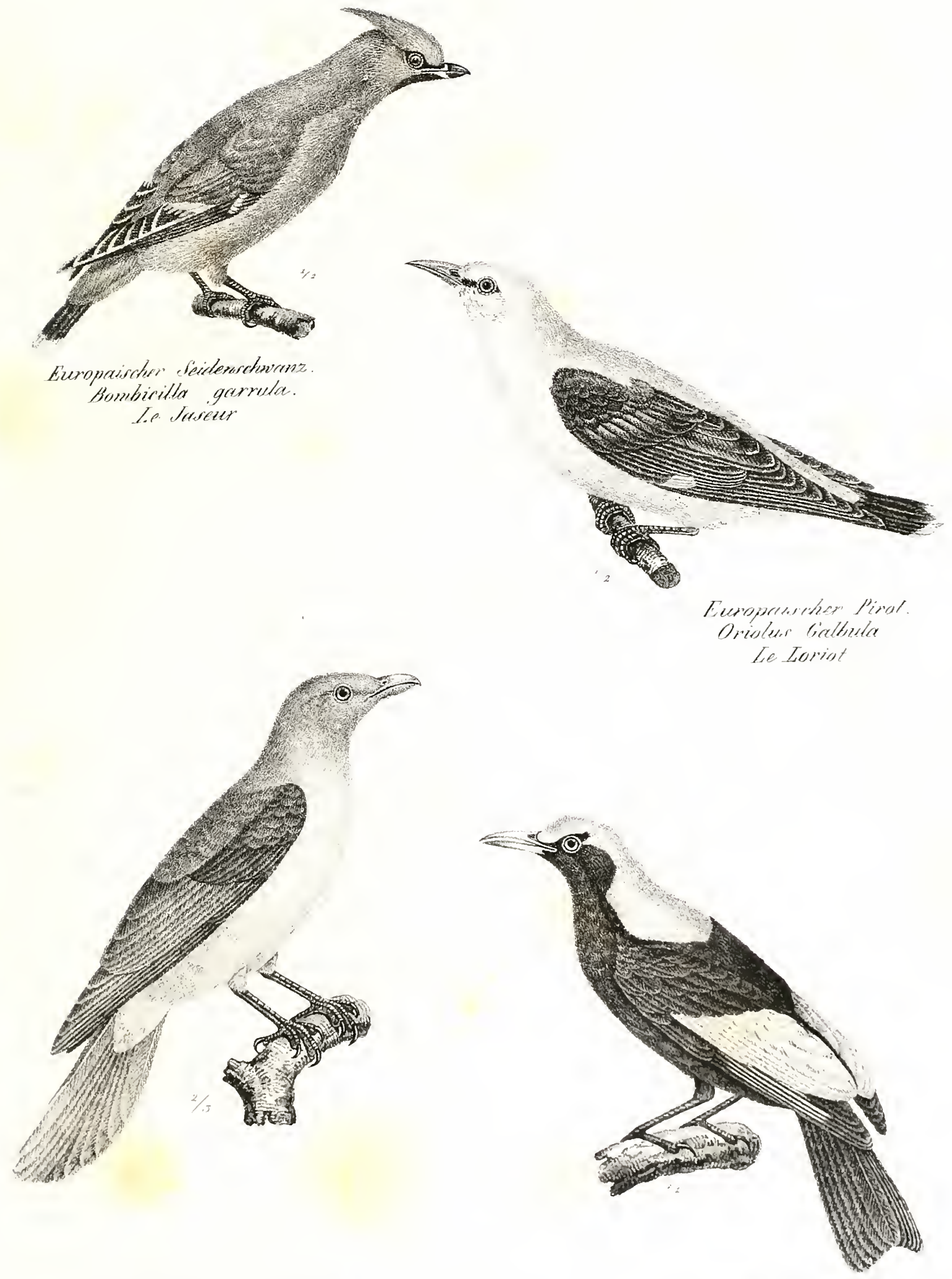

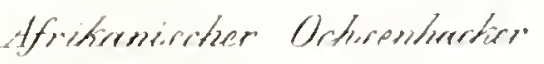
Prepliager erythenrhypetiu Pigue-bocuf bes-comil. 

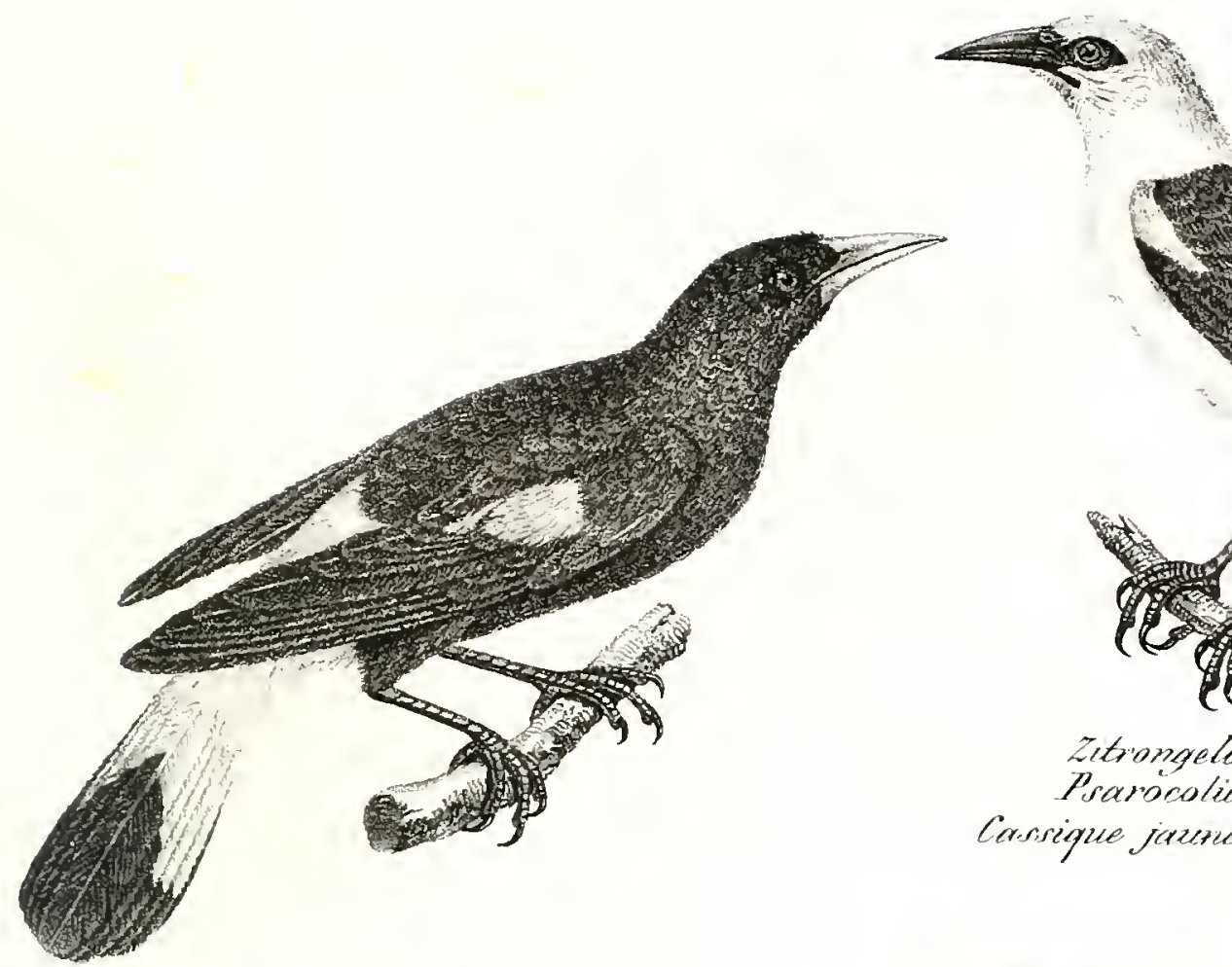

\section{Gelbruchige ftandohle}

Pirarocalius ectoronectus.

Consrigue joume dee Priéril.

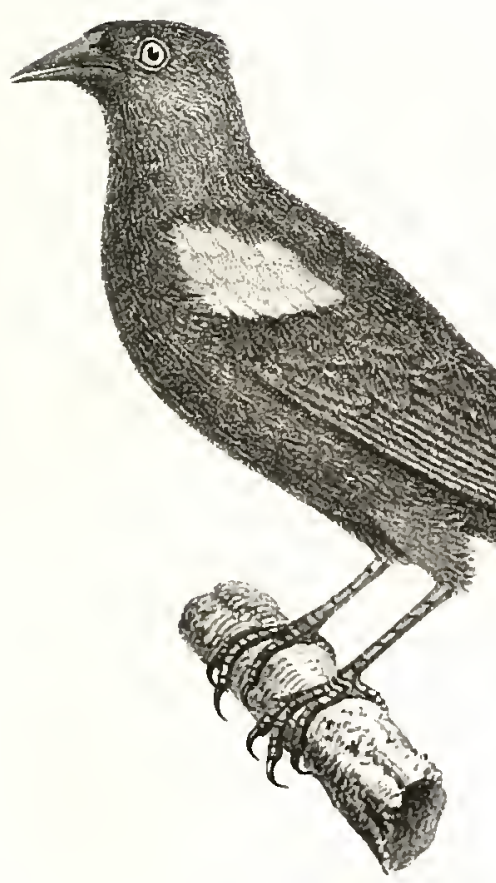

Bothflingline

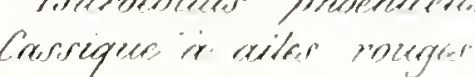

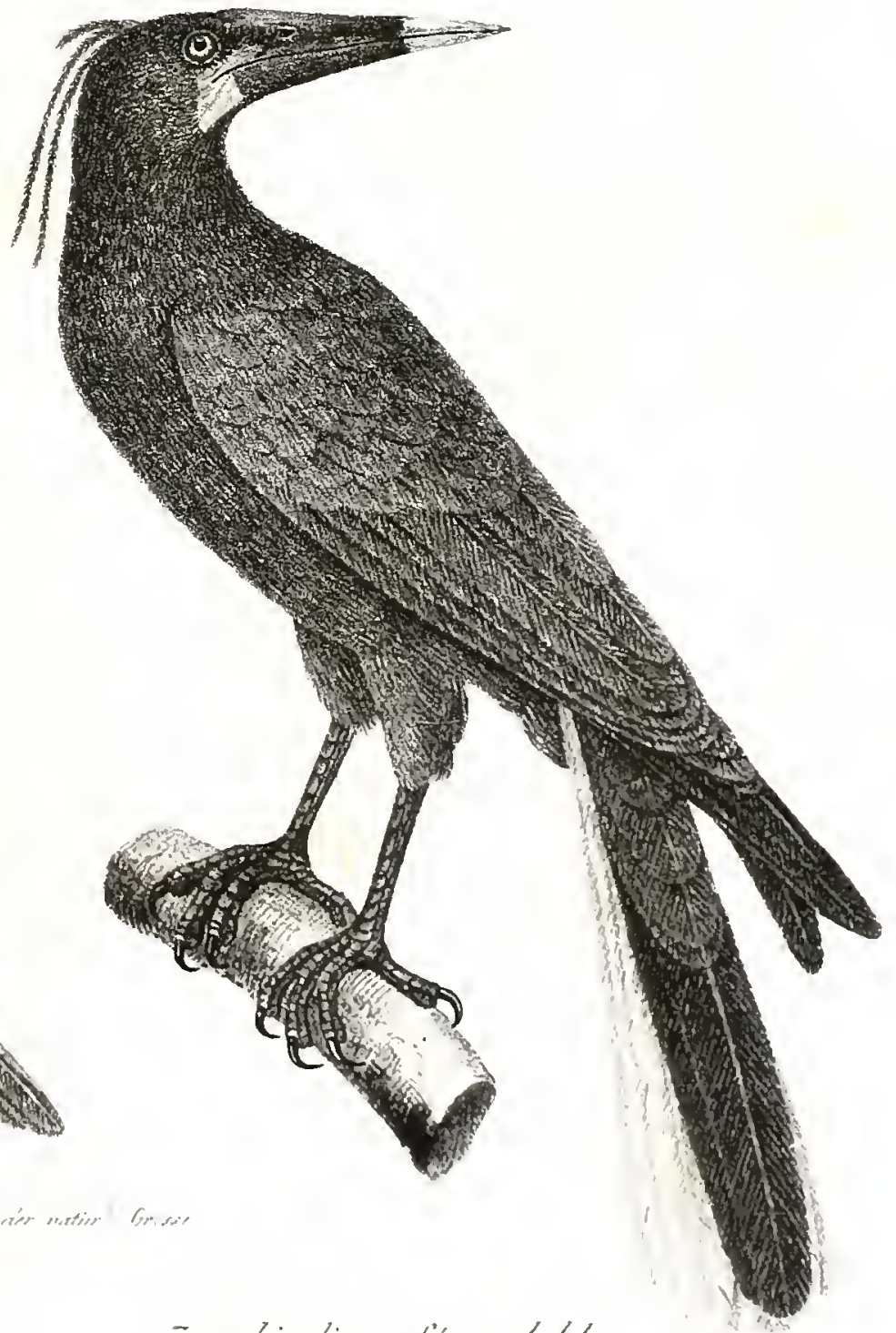

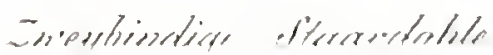

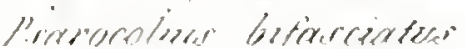

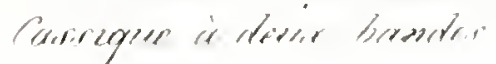



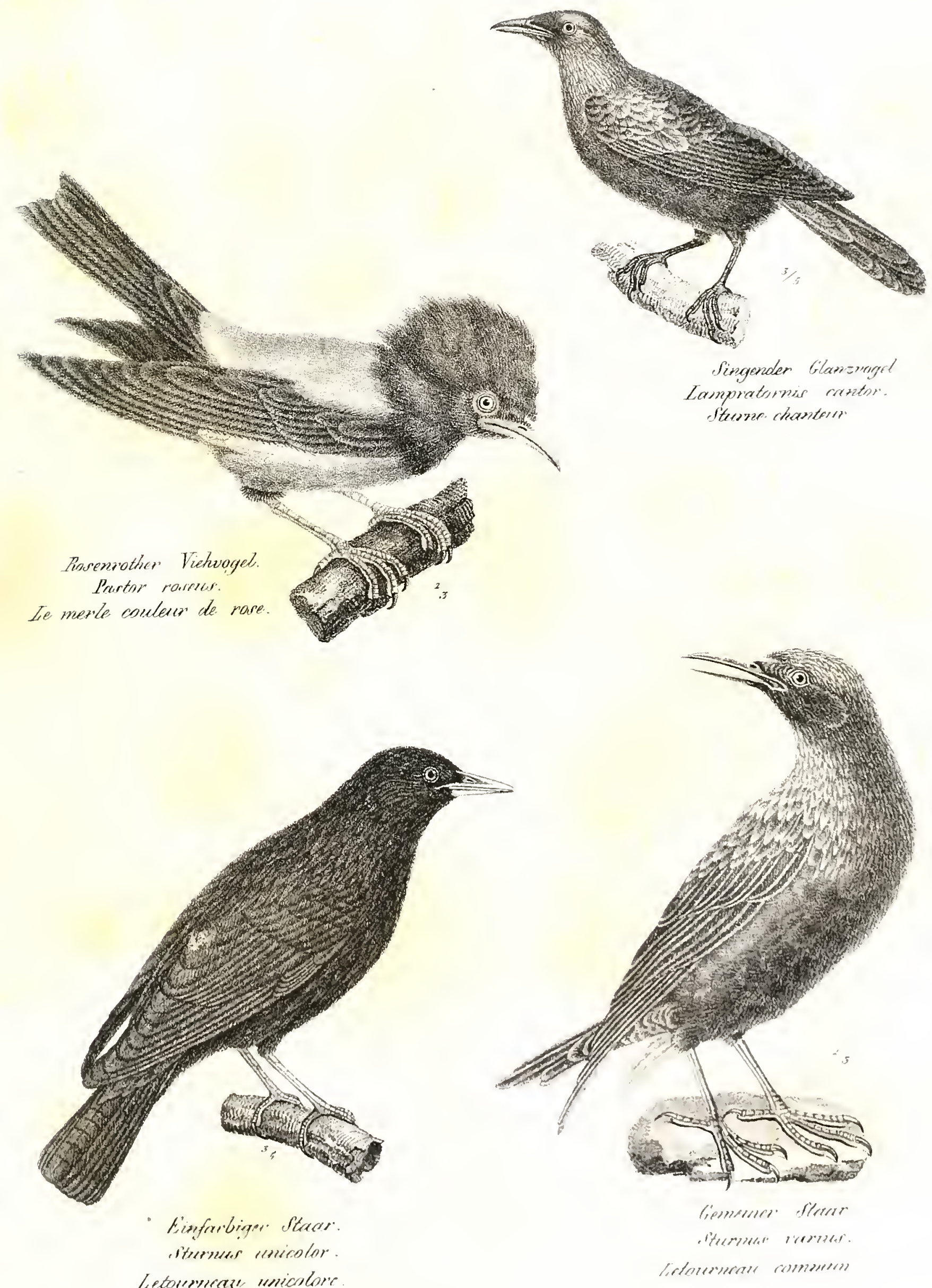

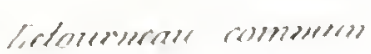



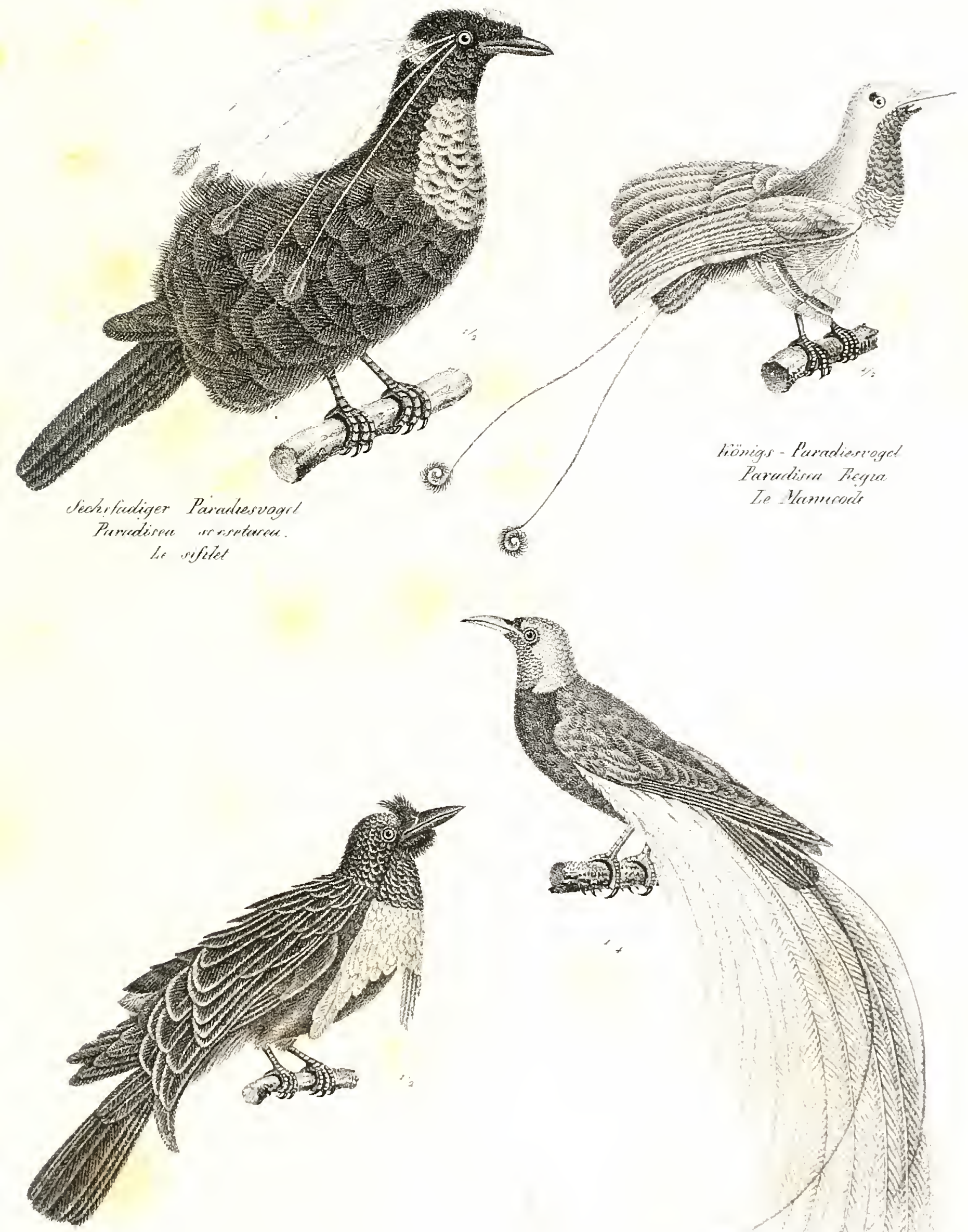

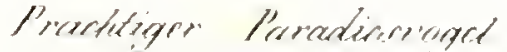
Percediren ersepuerlos

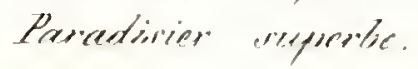

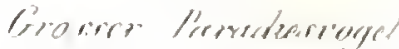

Preserdiseres repenters

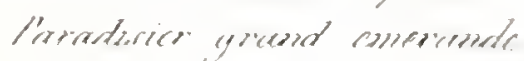



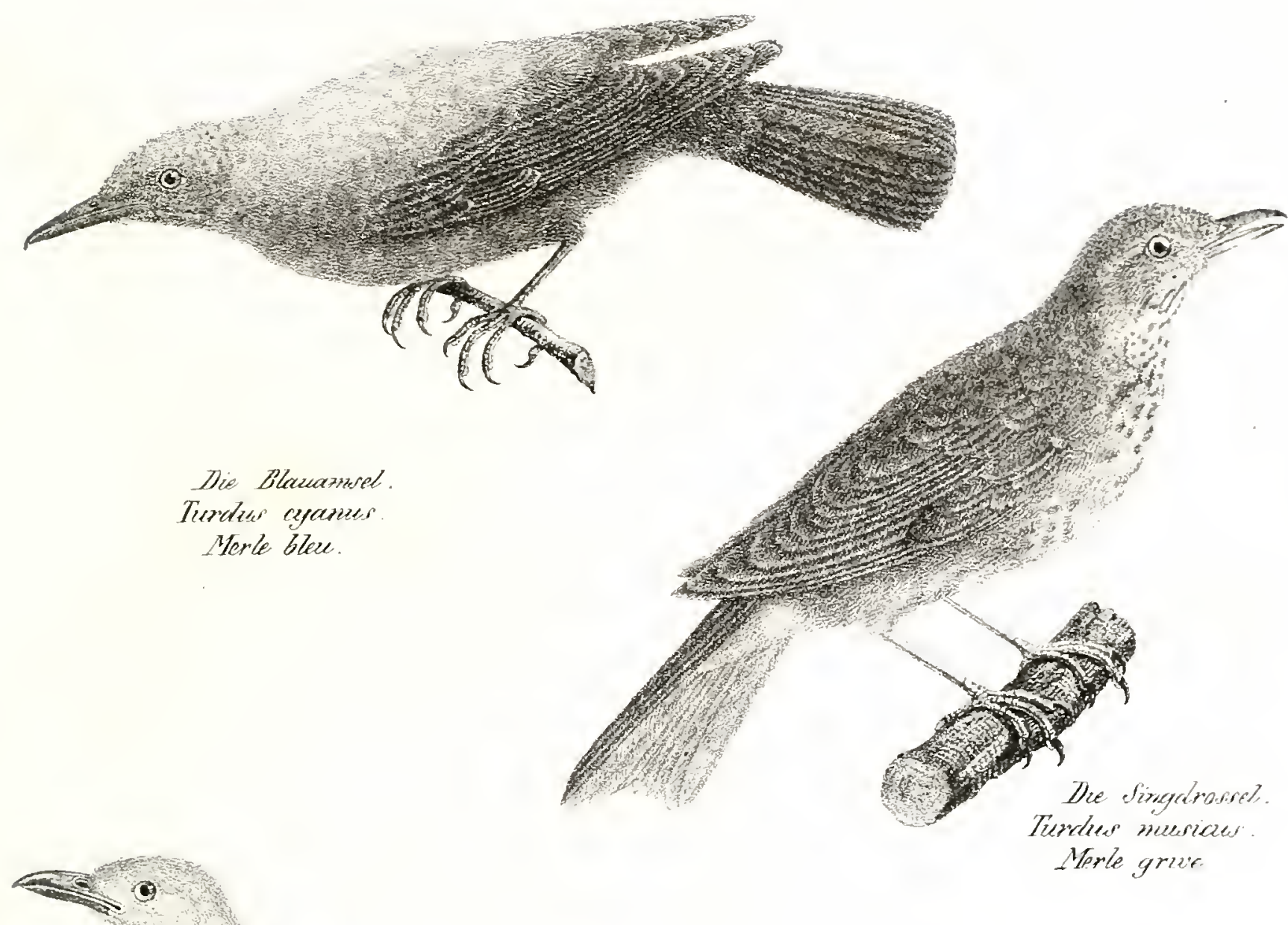

Merle grier.

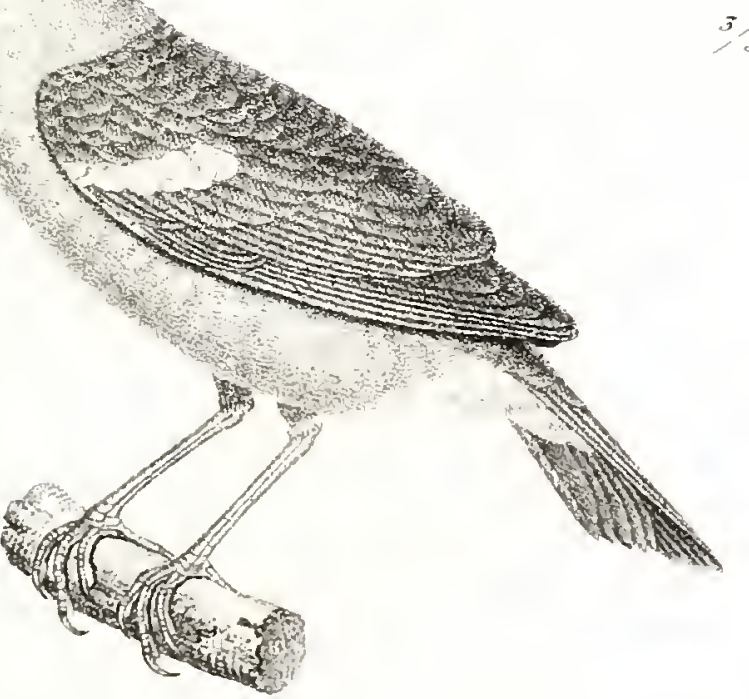

Grtbe Droseret

Tierdear citsimen.

Merle citrin

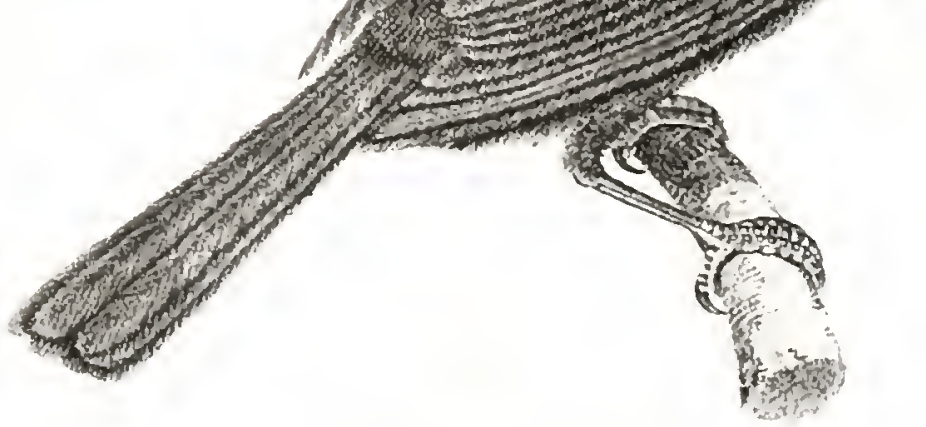

Dine gresereser Almerel

Teredien meredes

besels morer 


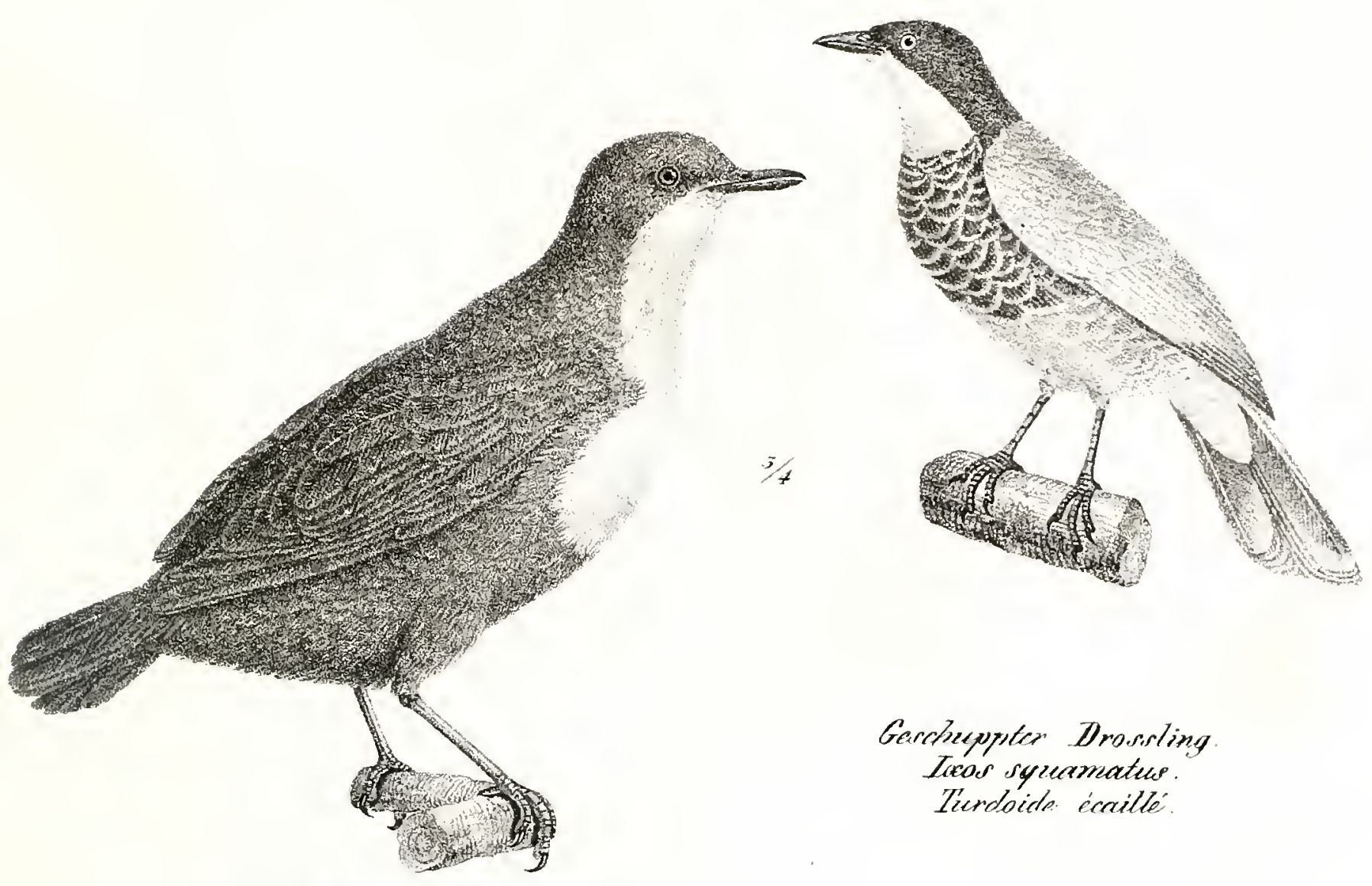

Gemeiner Wasserschnntane

Cincleas aquaticur
Gerchecupter Drosisling
ticas syuamatew.
Tierdoedto écrillé.
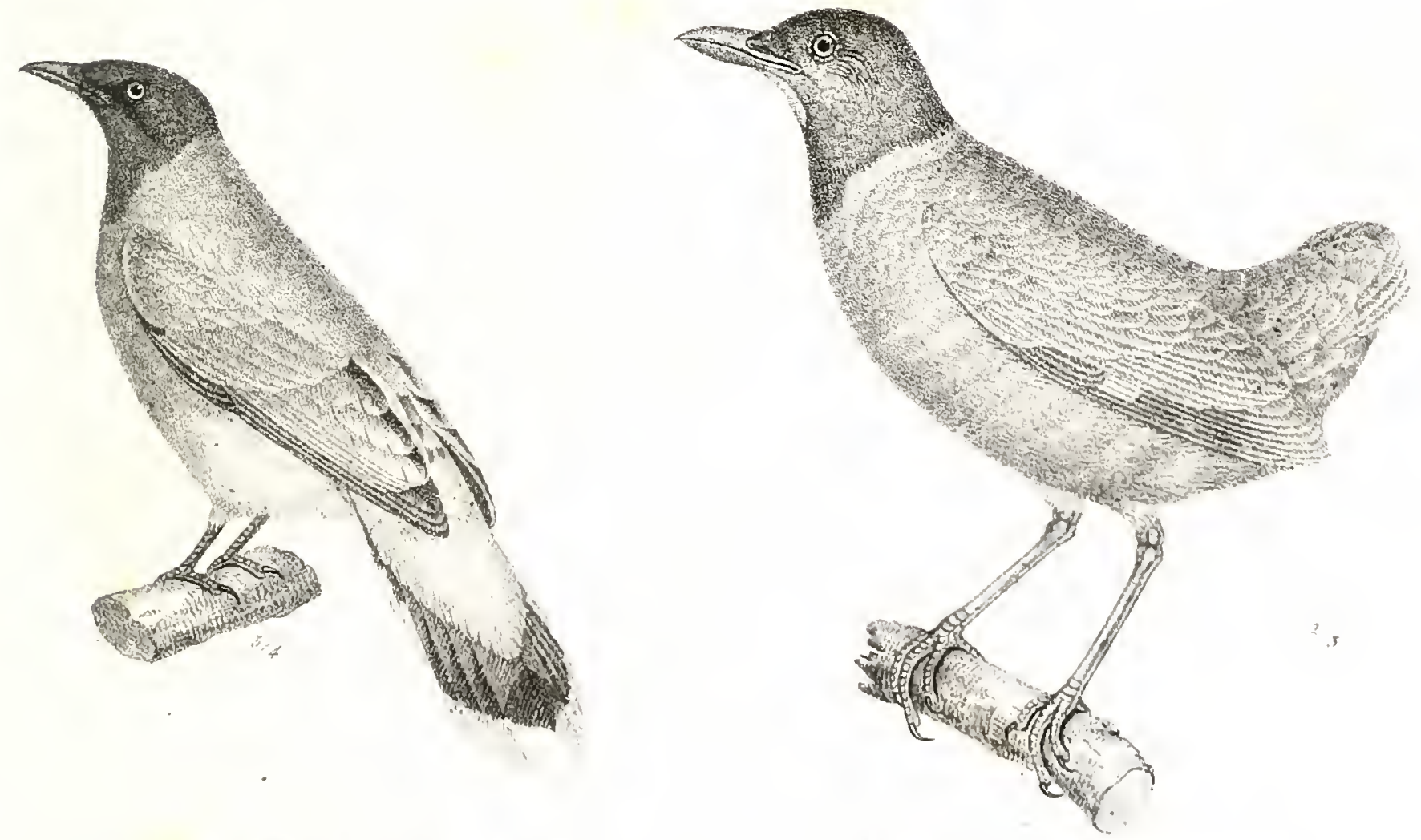

Draseling mit blauglanzenenten hinpt

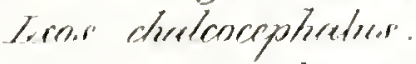

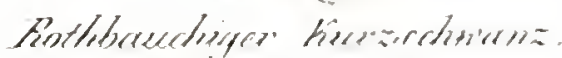

Teerdoiste cup-bronsr Rittre coytheorgrestor

Breeter it uestre ronge 


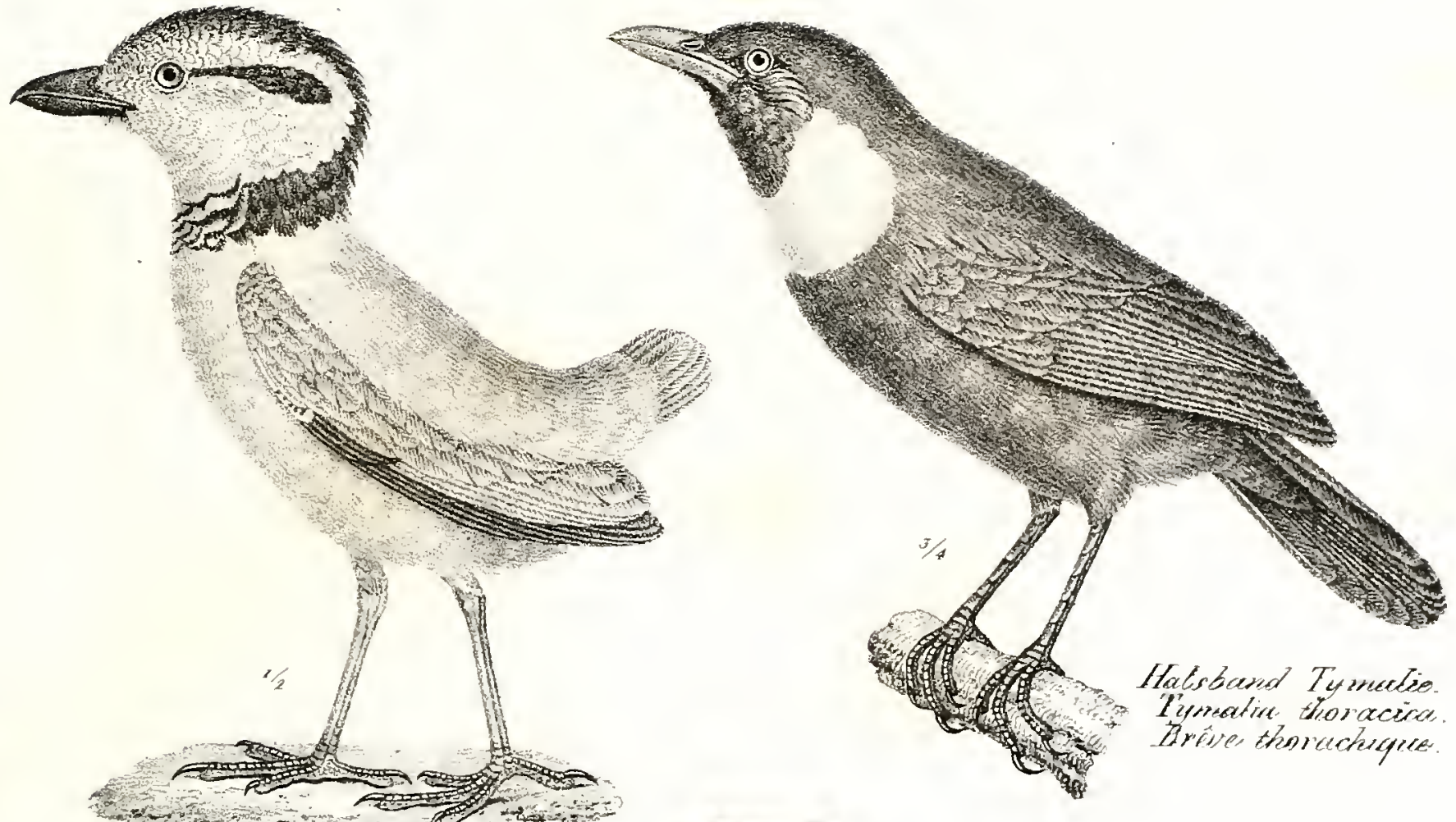

$$
\begin{gathered}
\text { Grosien hurzochesicus } \\
\text { Pitte gigos. } \\
\text { Brése géart. }
\end{gathered}
$$

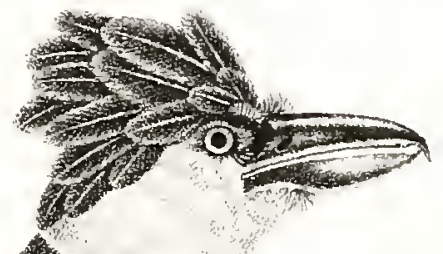

I $5-27$

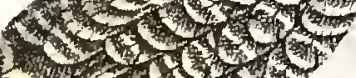

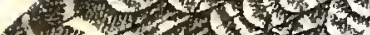

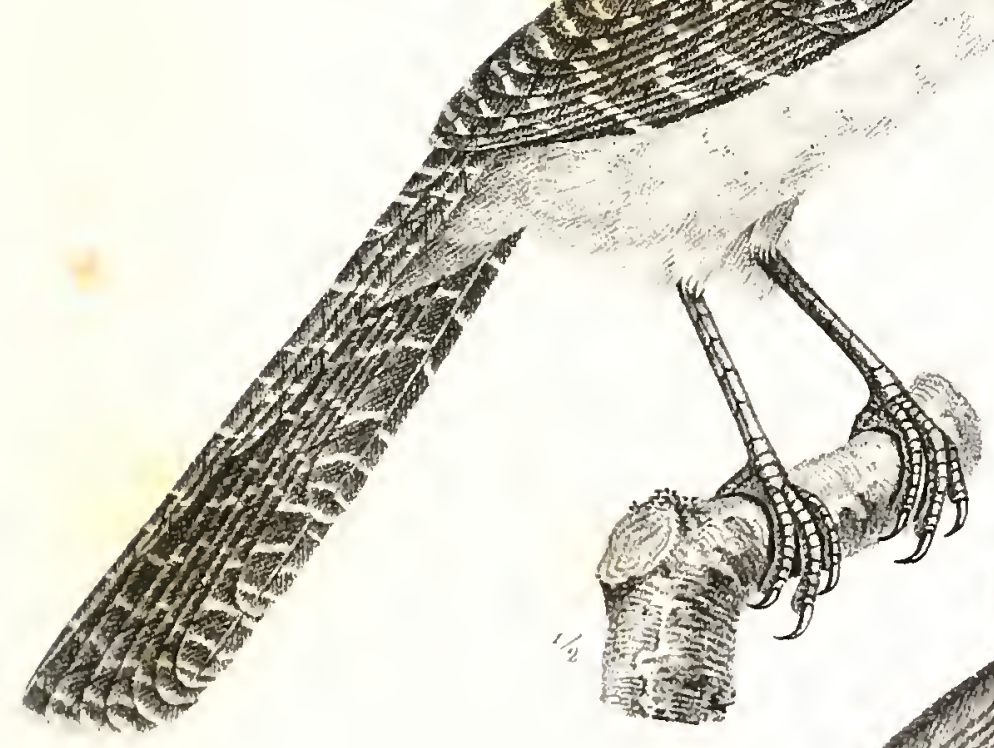

$$
\begin{aligned}
& \text { Geastreiftes Vrunges. }
\end{aligned}
$$

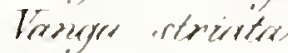

$$
\begin{aligned}
& \text { Tresuger merege }
\end{aligned}
$$

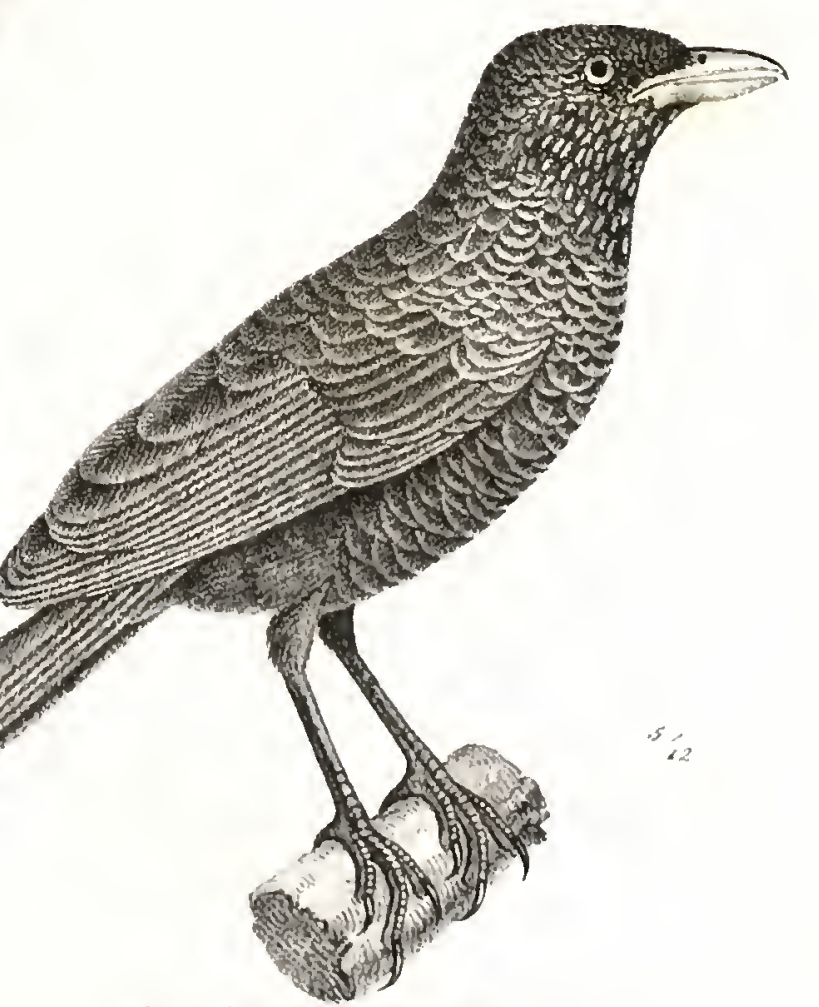

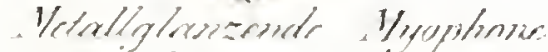

deopremener metritlerses

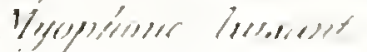




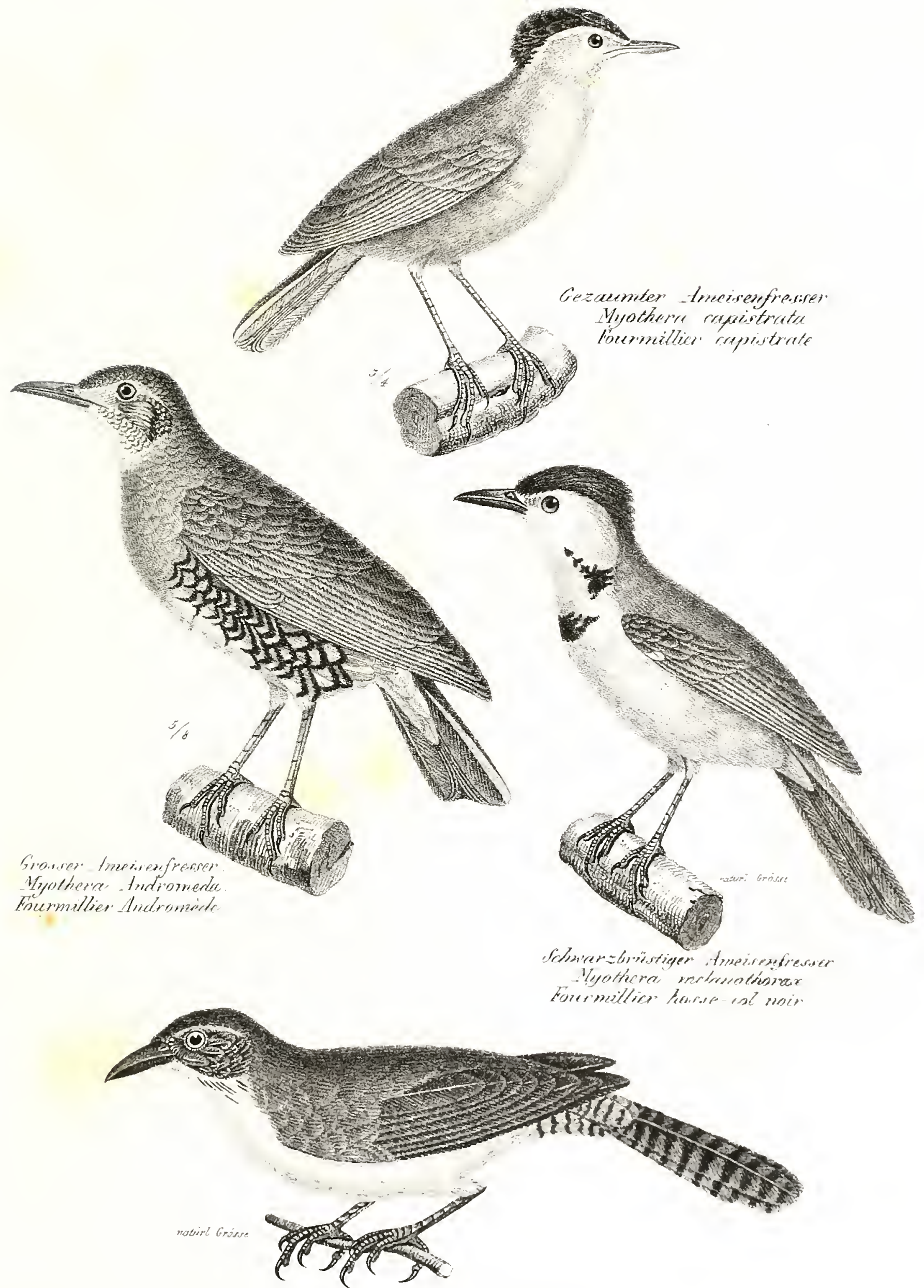

Anecisenfresions lineresea

Mugritlewa conirega.

Lis Couresse 

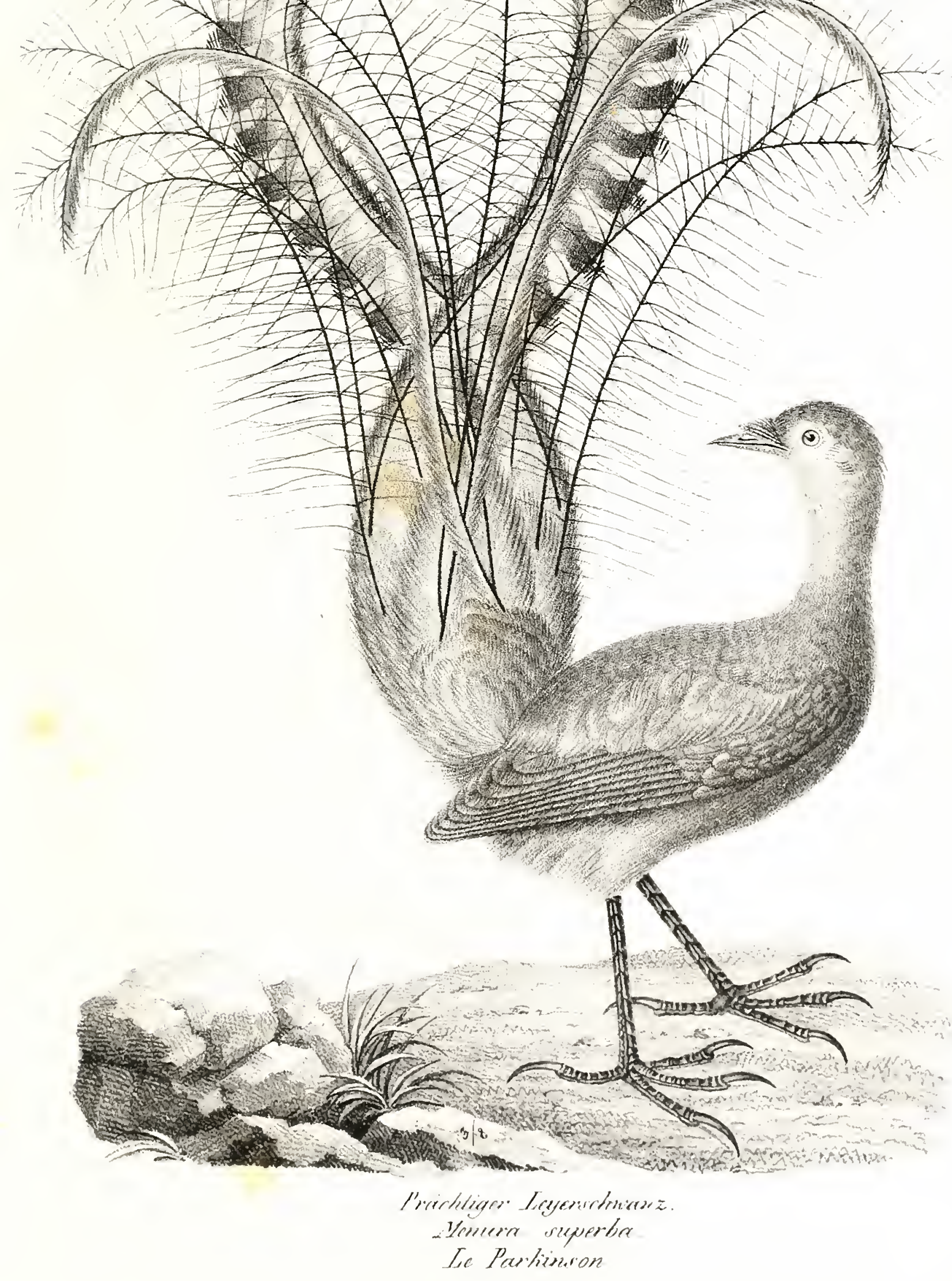


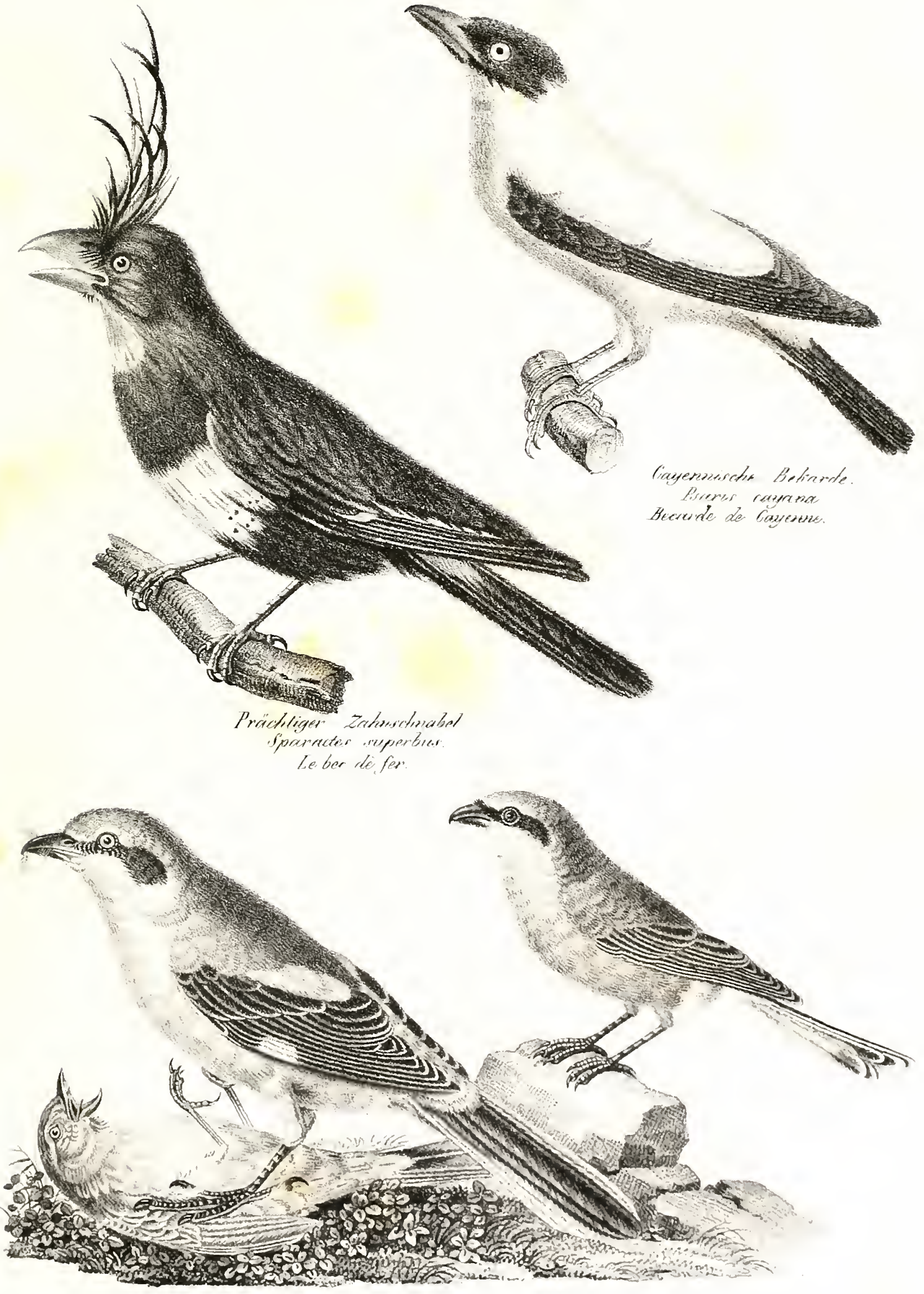

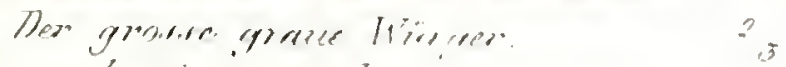

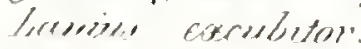

$$
\begin{aligned}
& \text { lese-greiseles griere. } \\
& \text { Dre. Anewereretions. }
\end{aligned}
$$

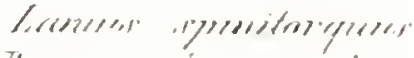

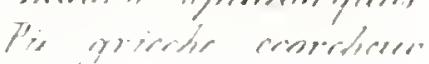



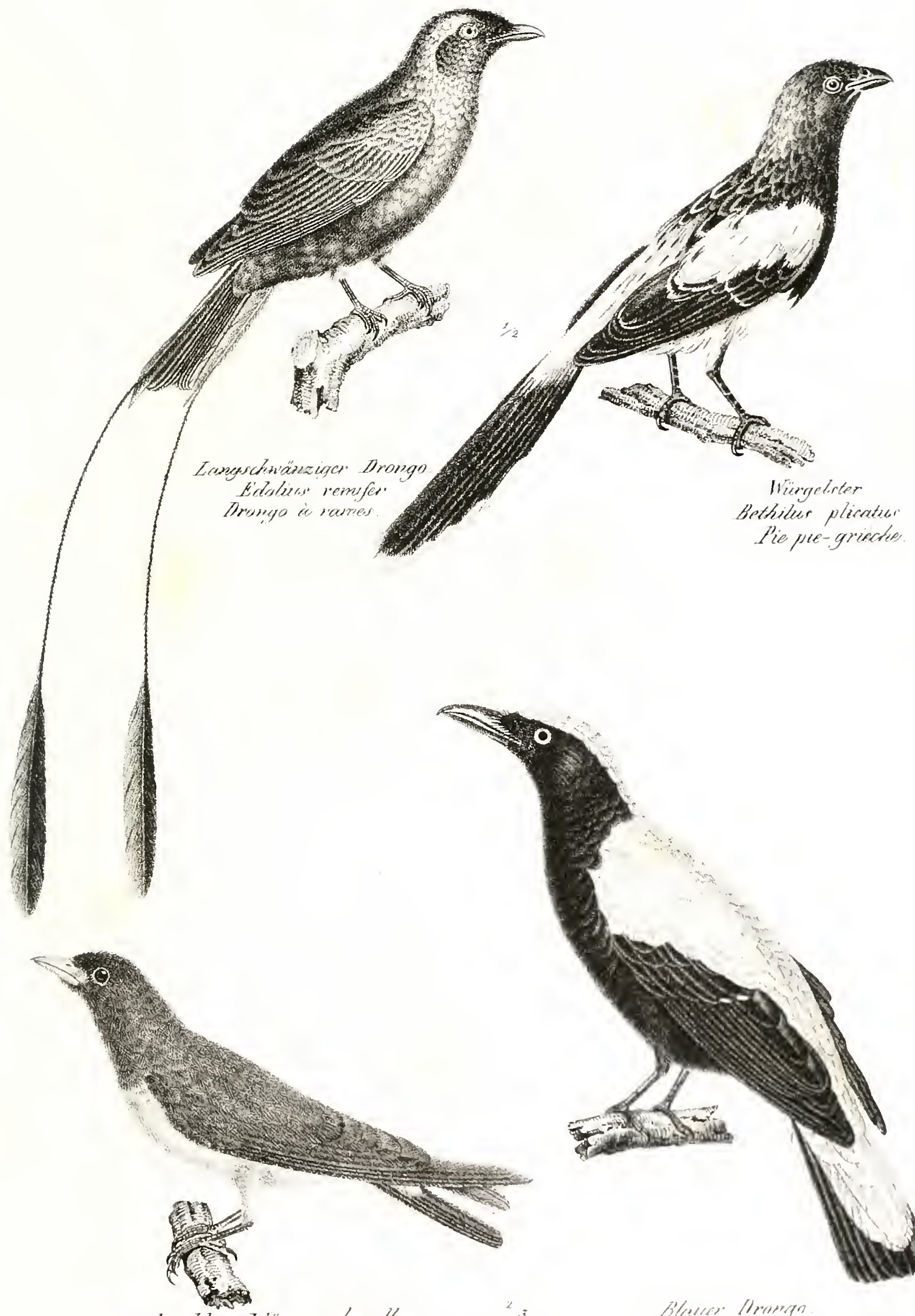

Weisesshmablege Whirgesirelinvalle

Ocypoterver leussmynecher. Lexngrayes in bec blesed.

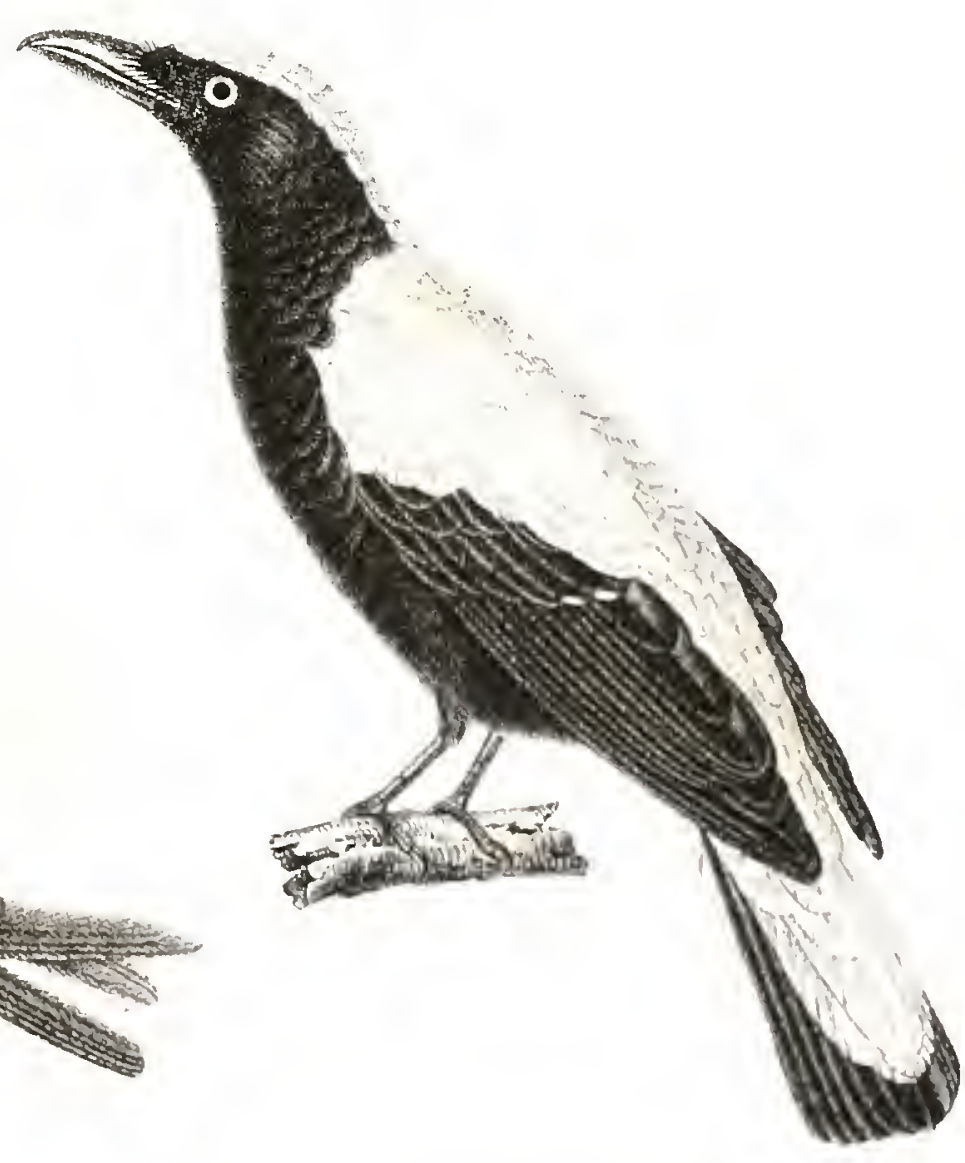

Blonese Mronerson

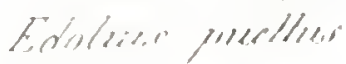

Drounero usere. 


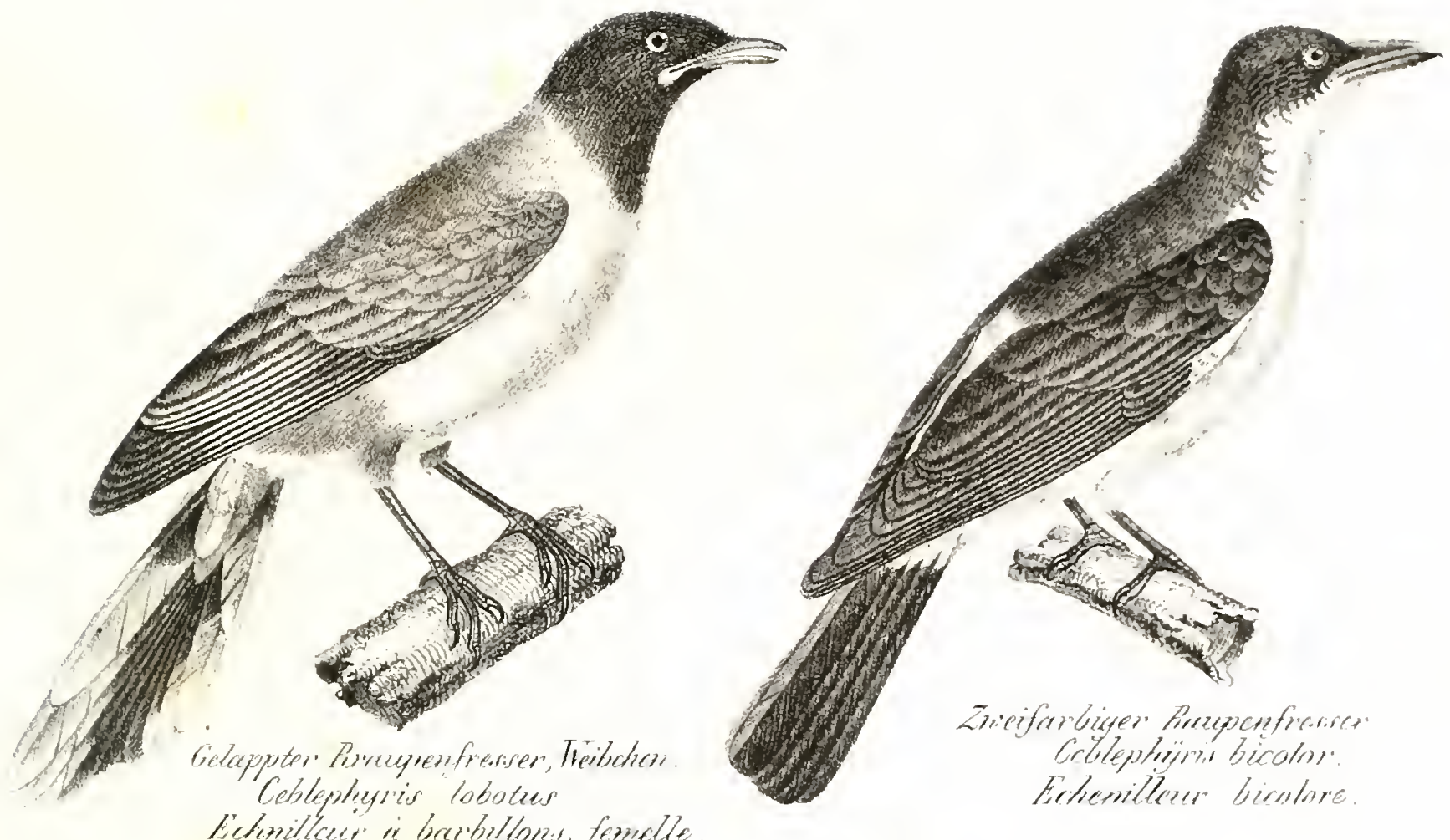
fetumilcer is barbithon. femelle

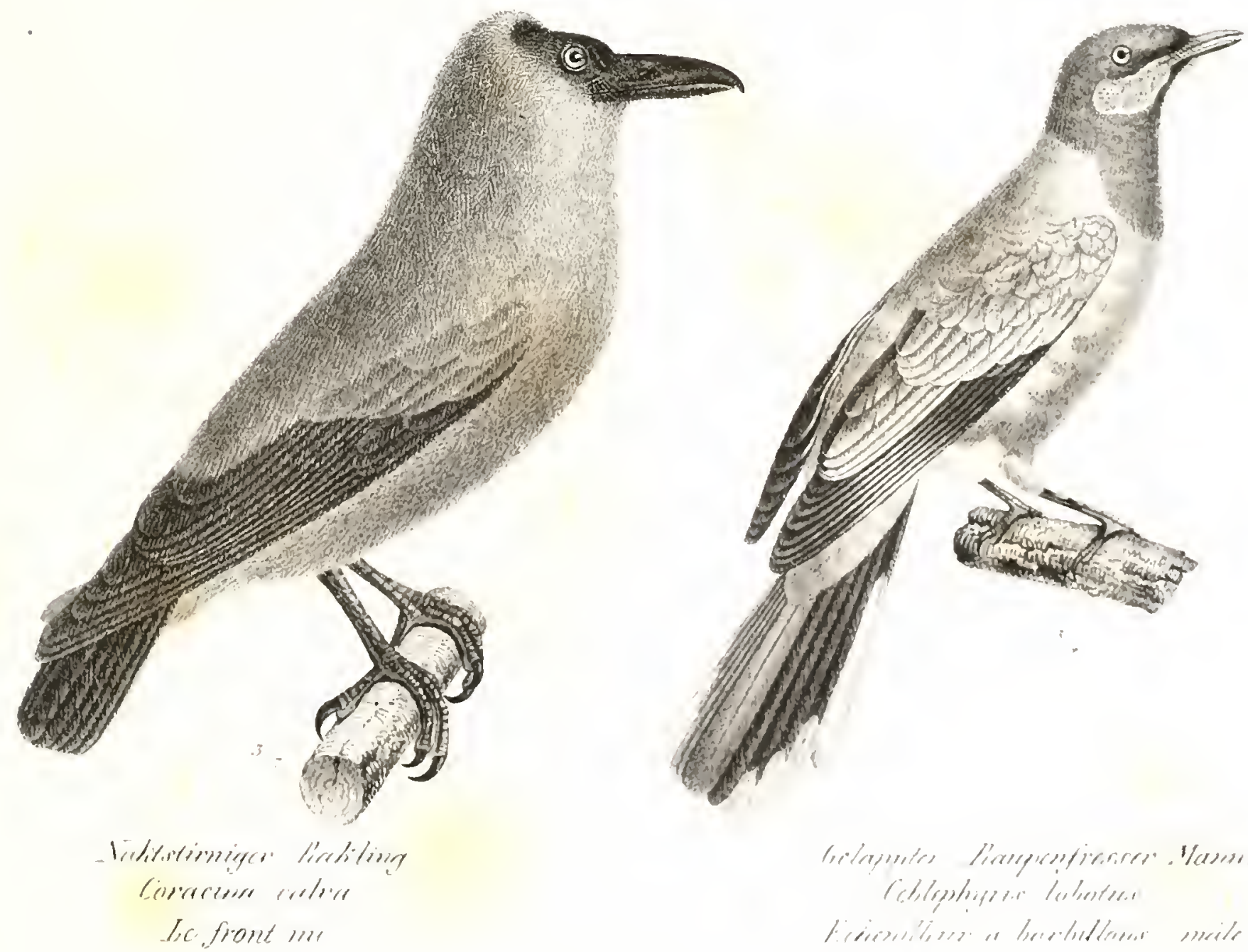



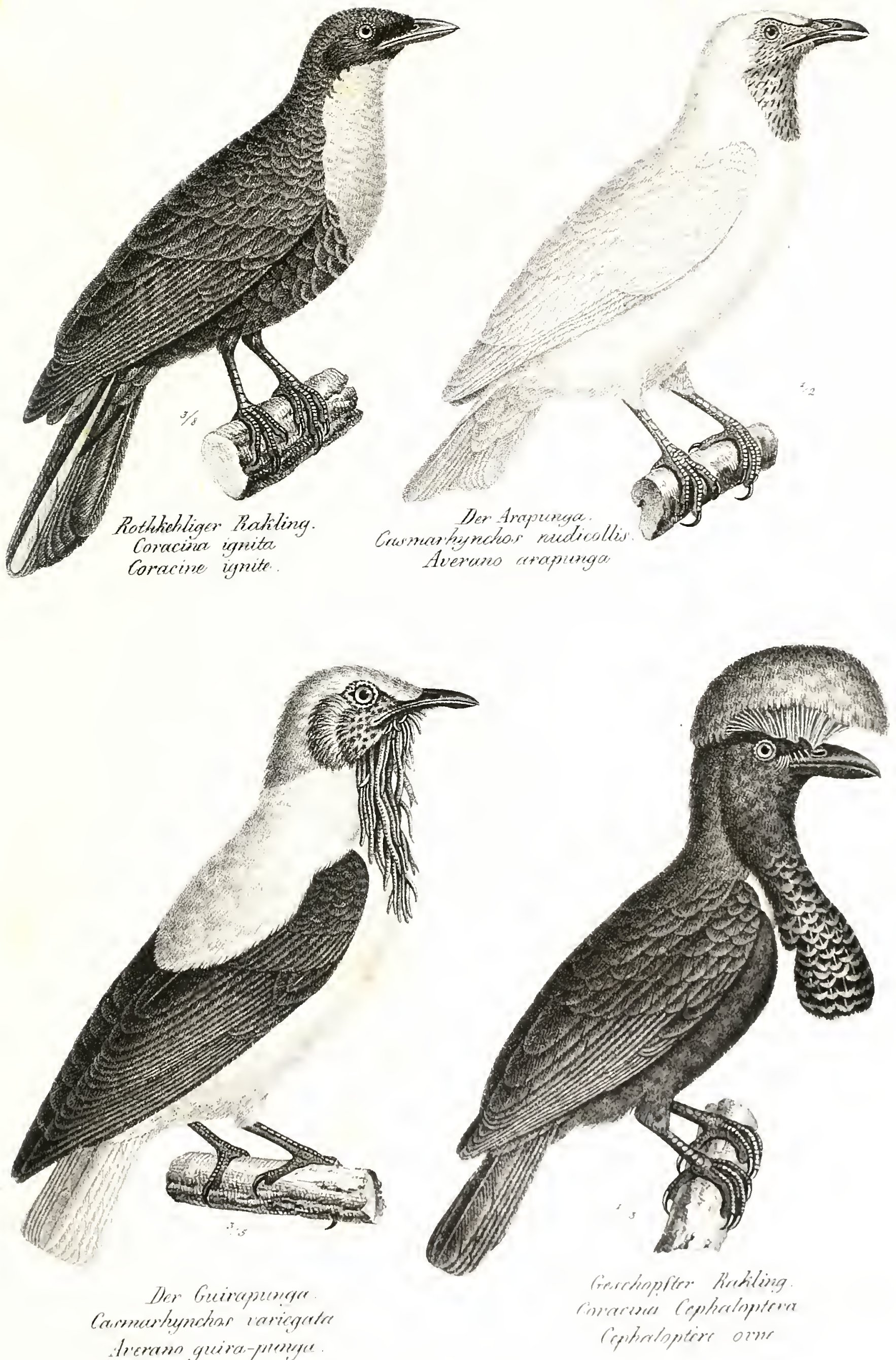


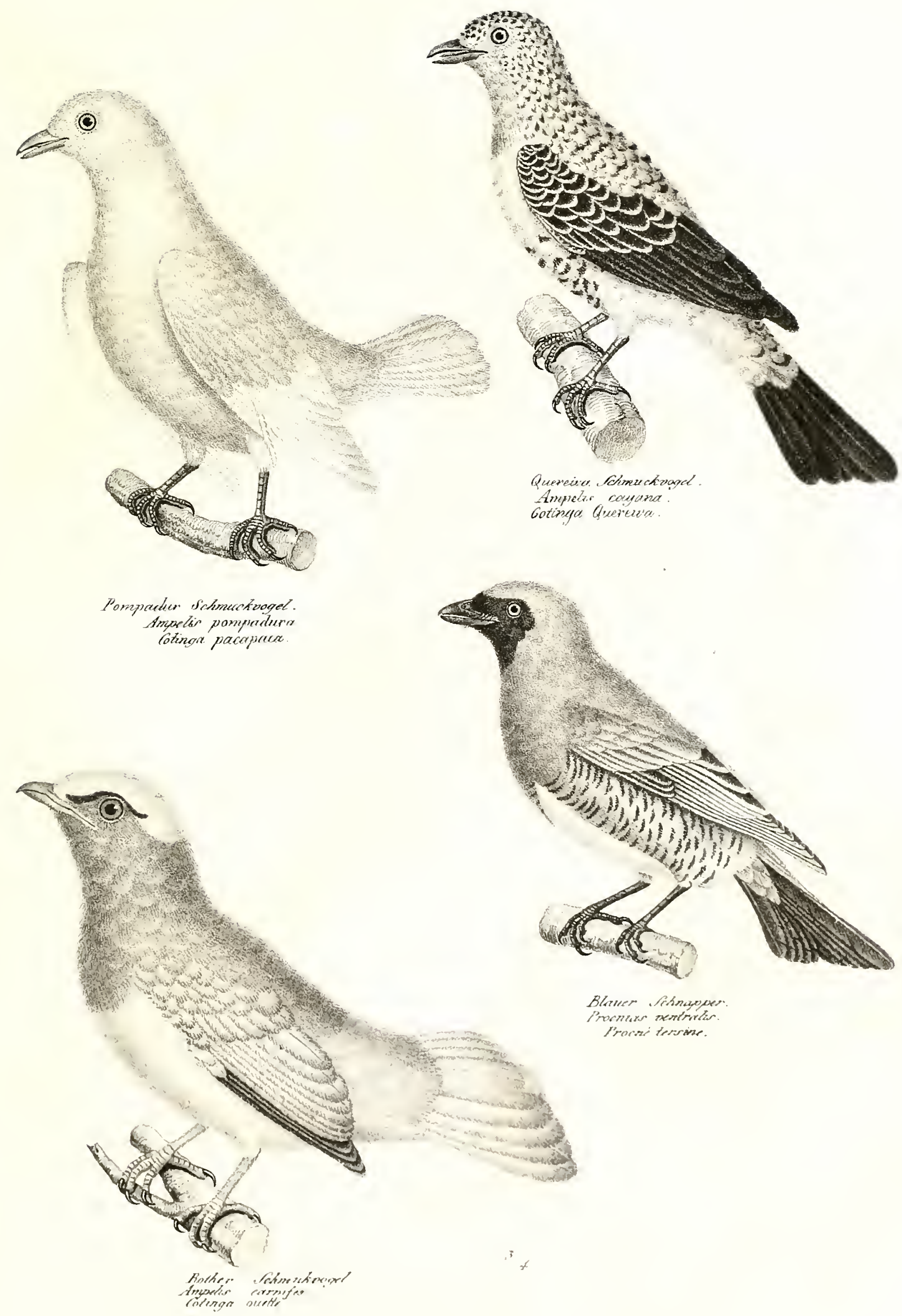


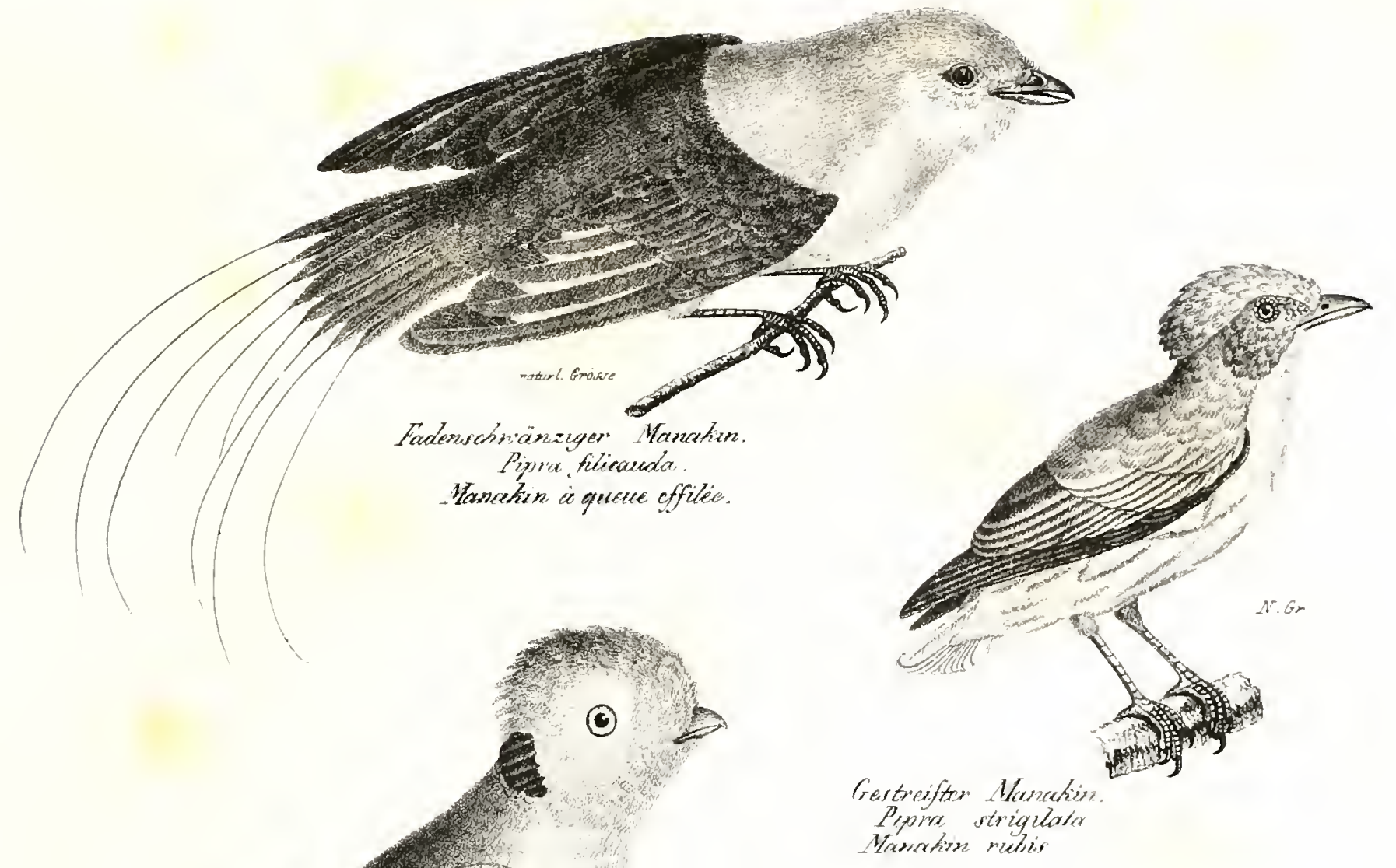

Grüner Felsenchation.

Prupicalo virveder. Rinepicales verdisr.

Gestreifter Acuncerist

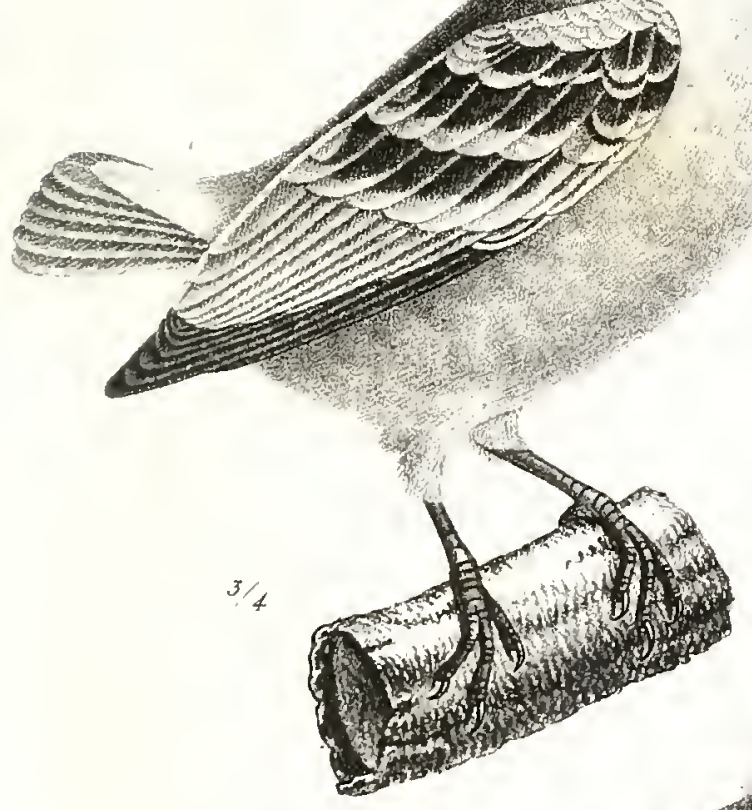

-
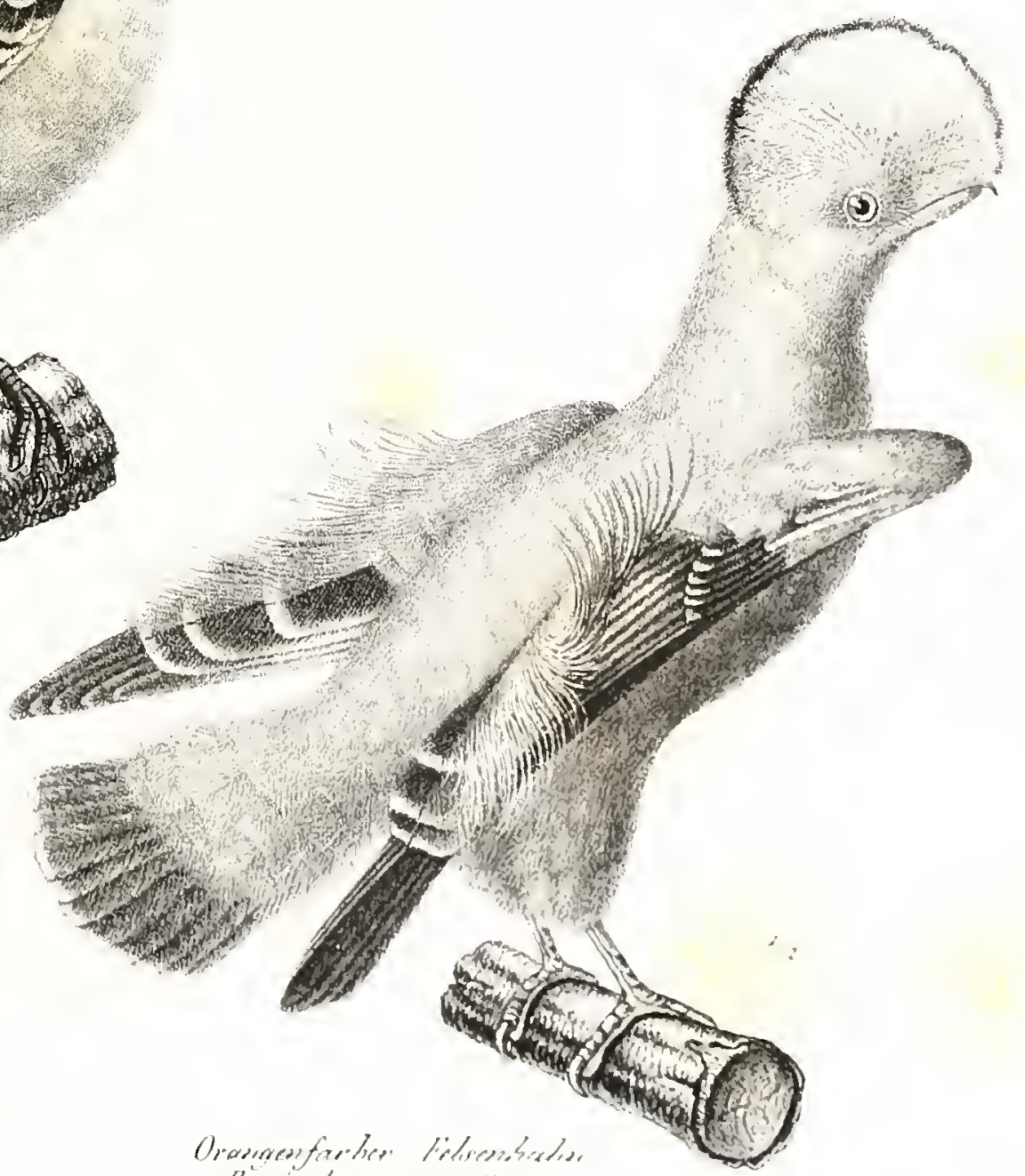

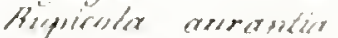

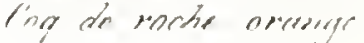




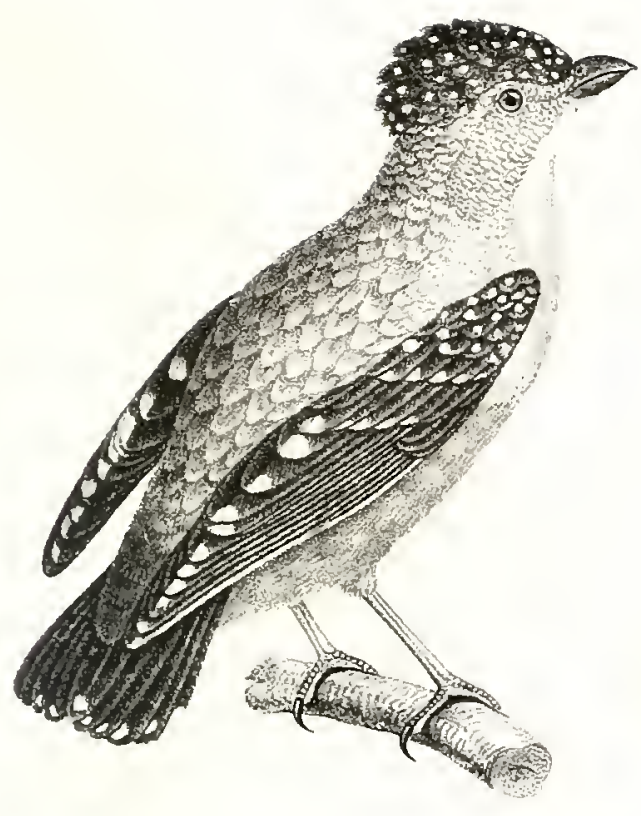

Punfitinter Panthorvogel

Pardicloters presectatiens

Pardiclobe pountelle

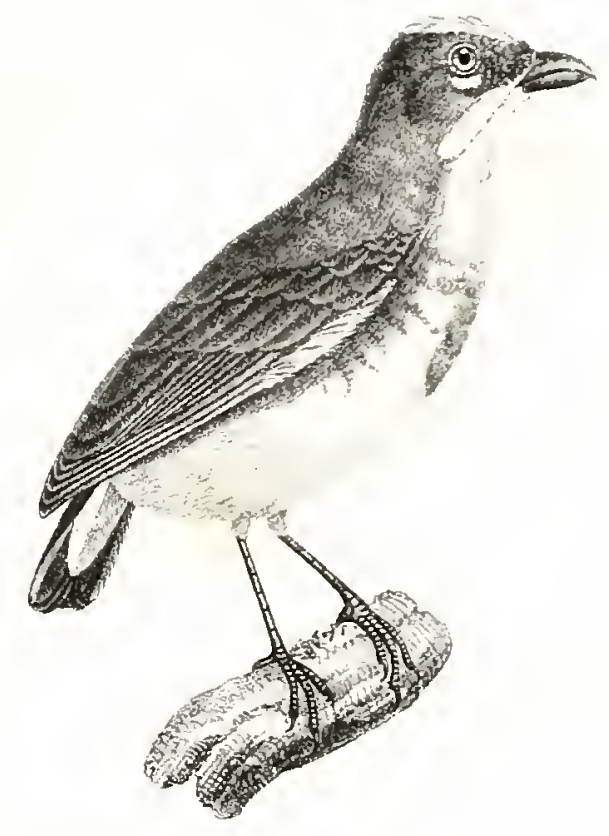

Termundetwe Pantherpogel.

Promblozien perranariar

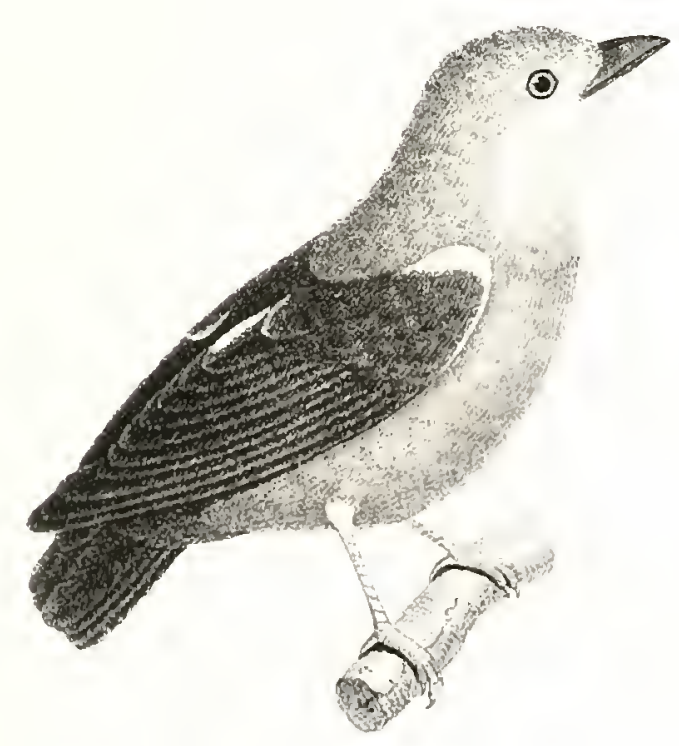

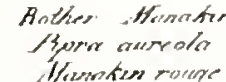

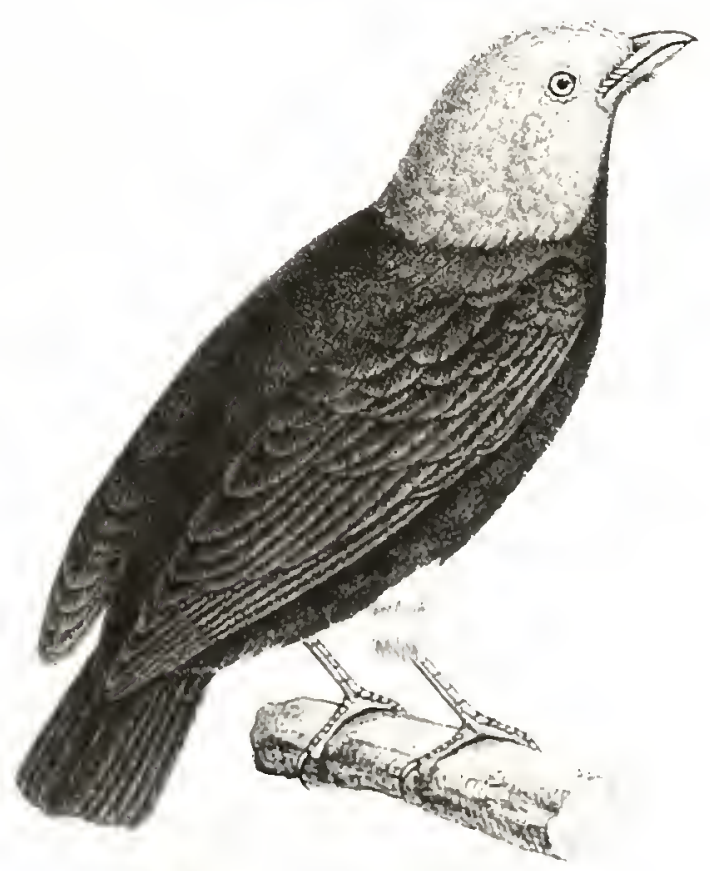

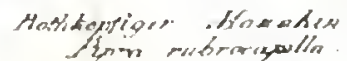

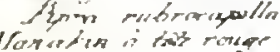



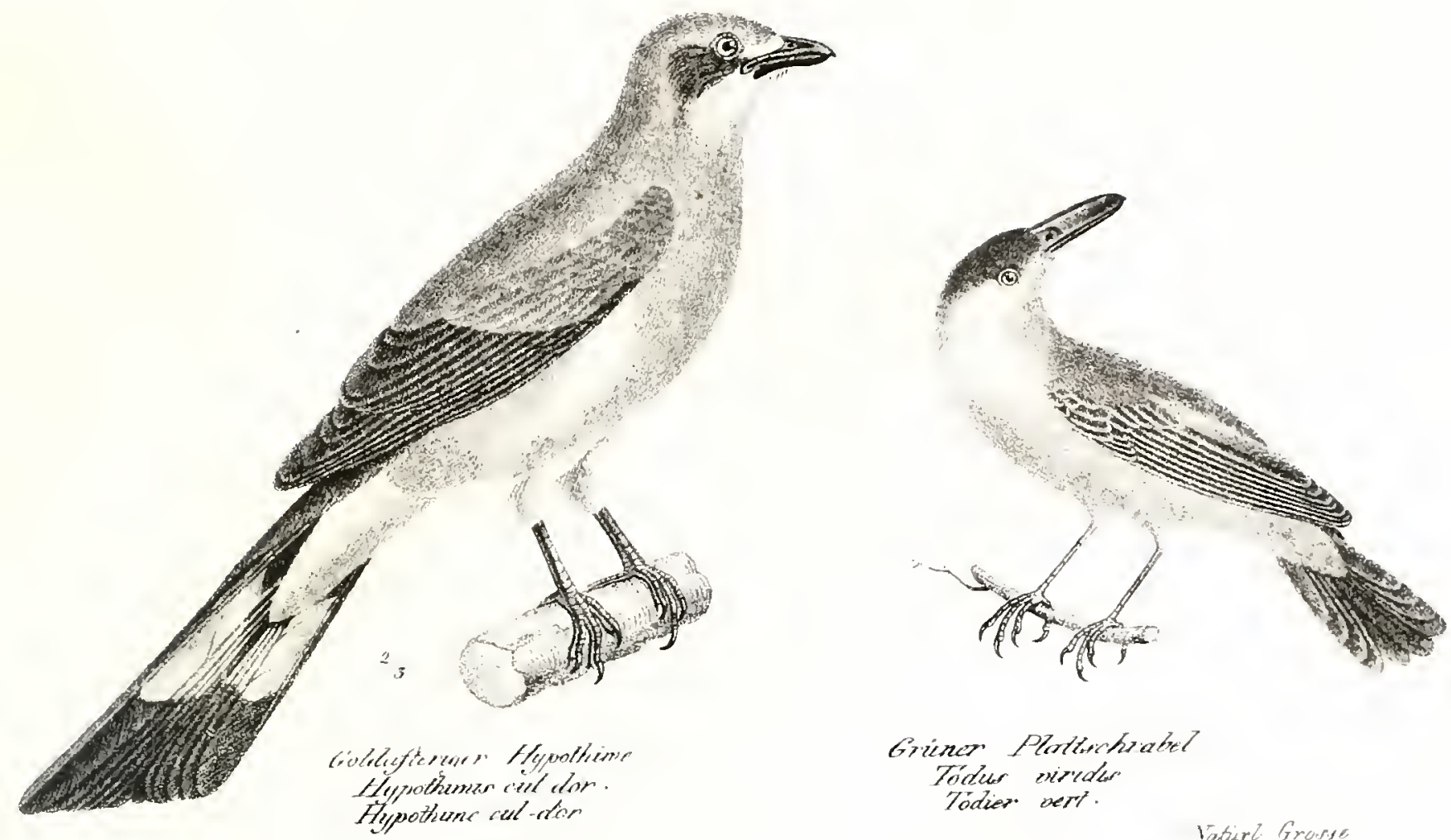
Grinor Plenturhiabel
Tódur evireder

Vationt Groses
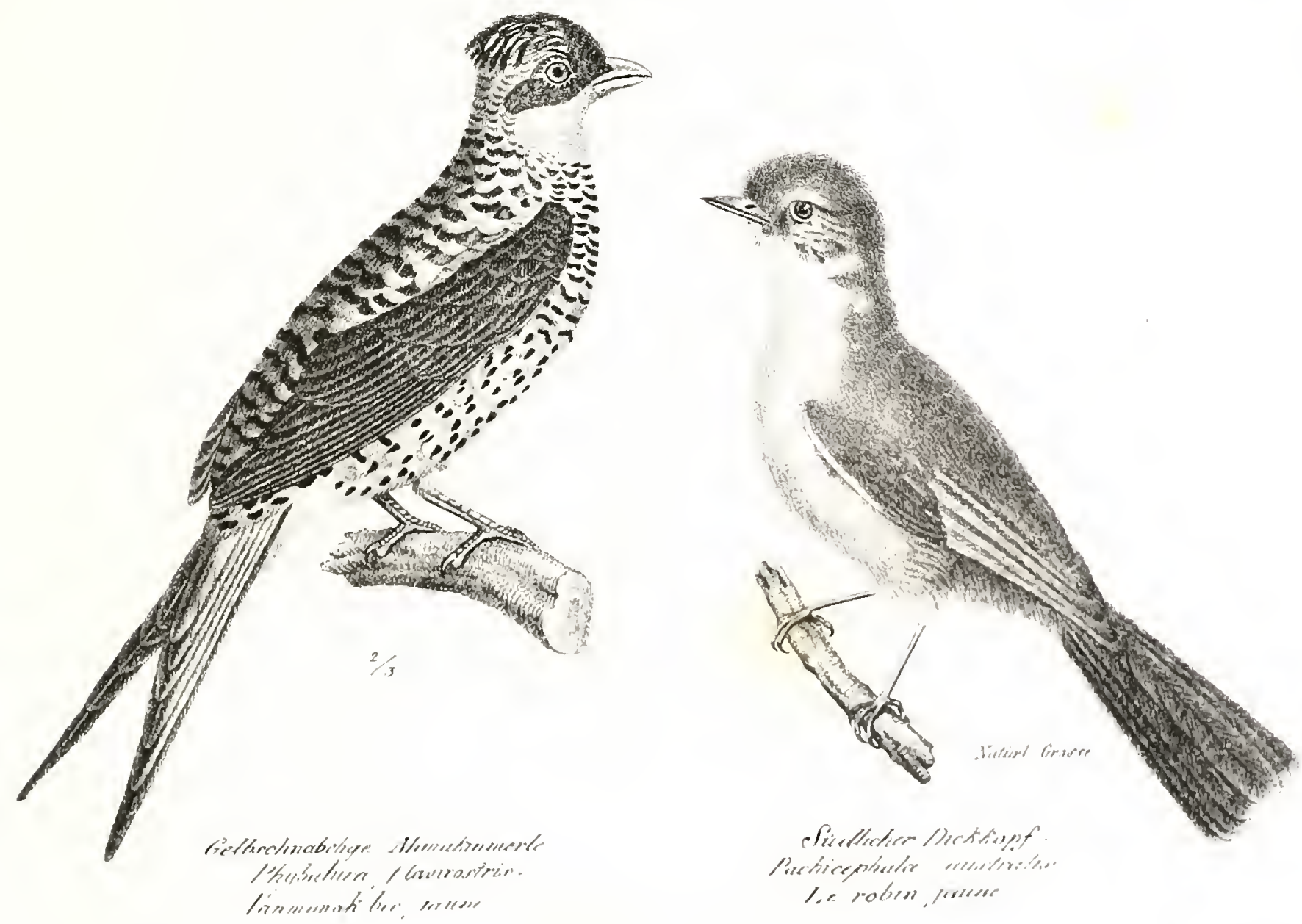

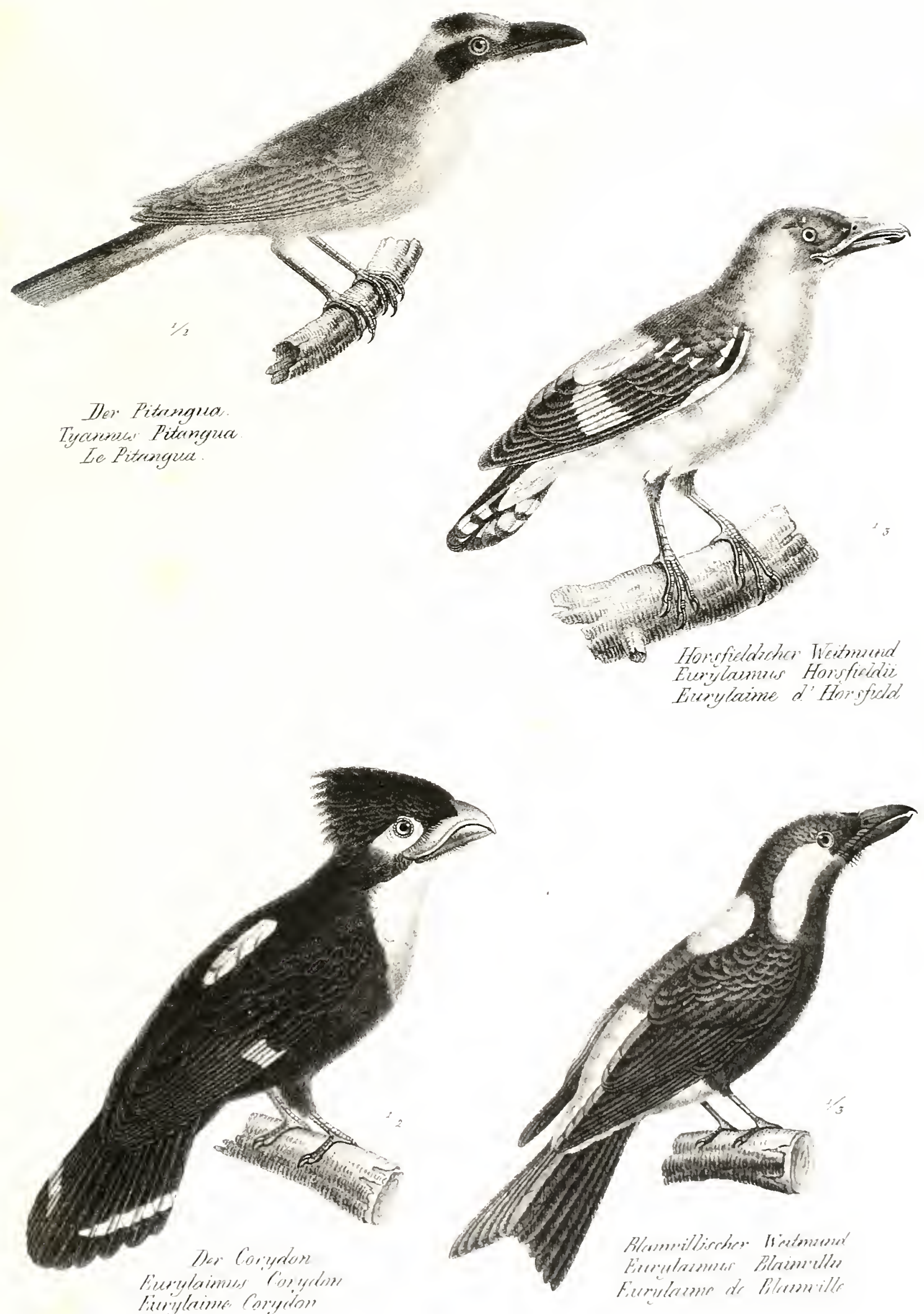
36.

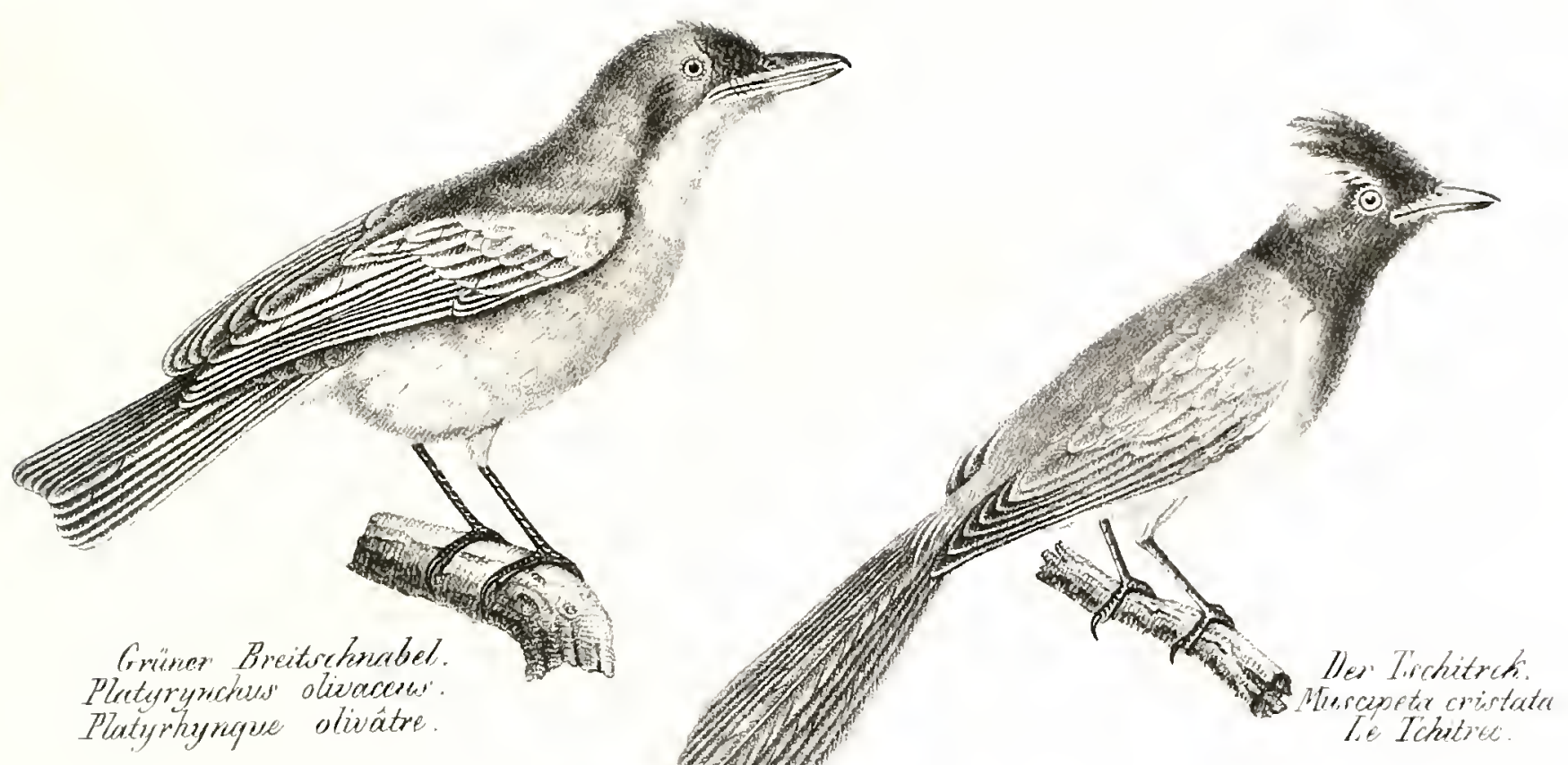

Platisinanguse olivatre.

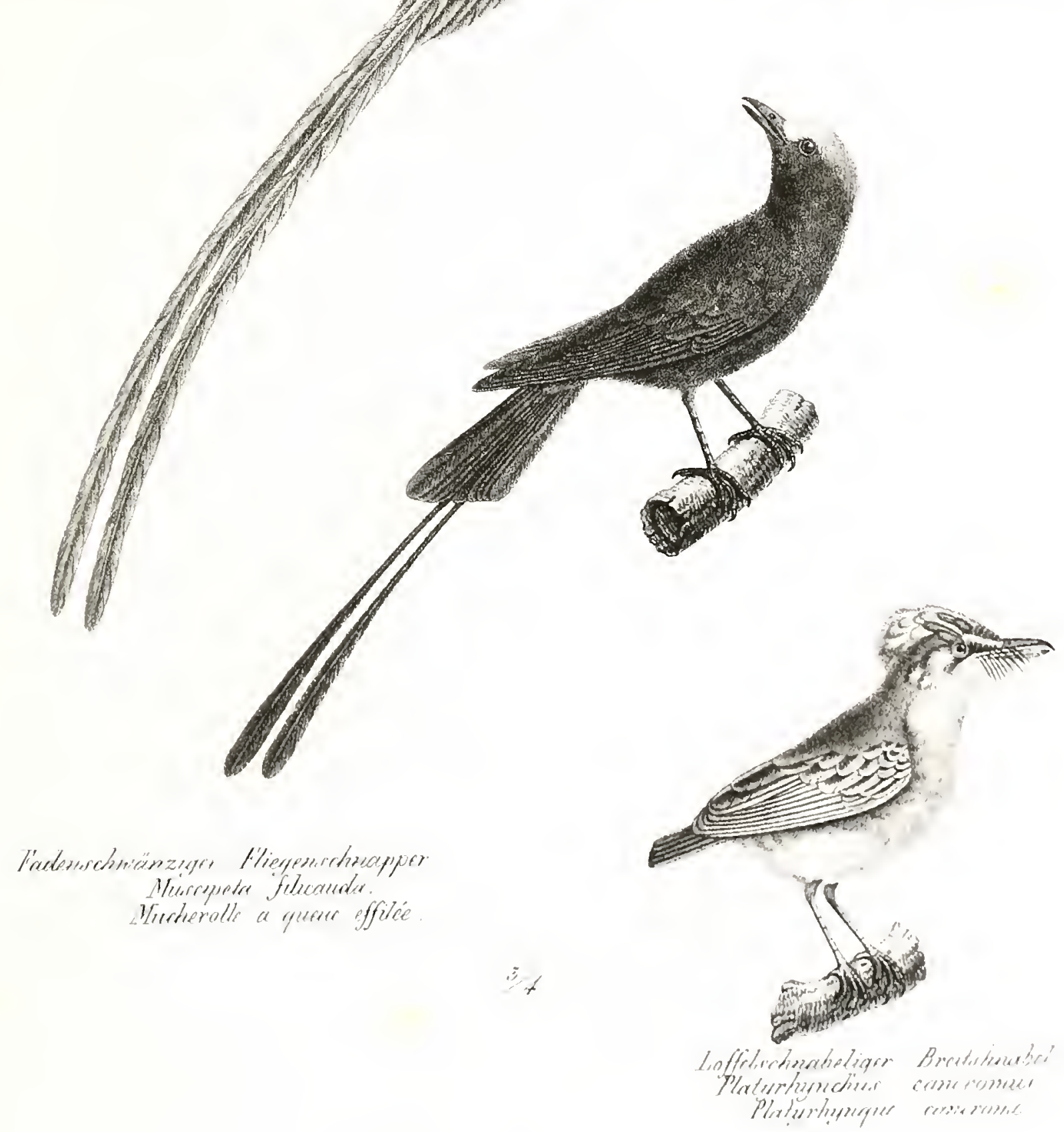




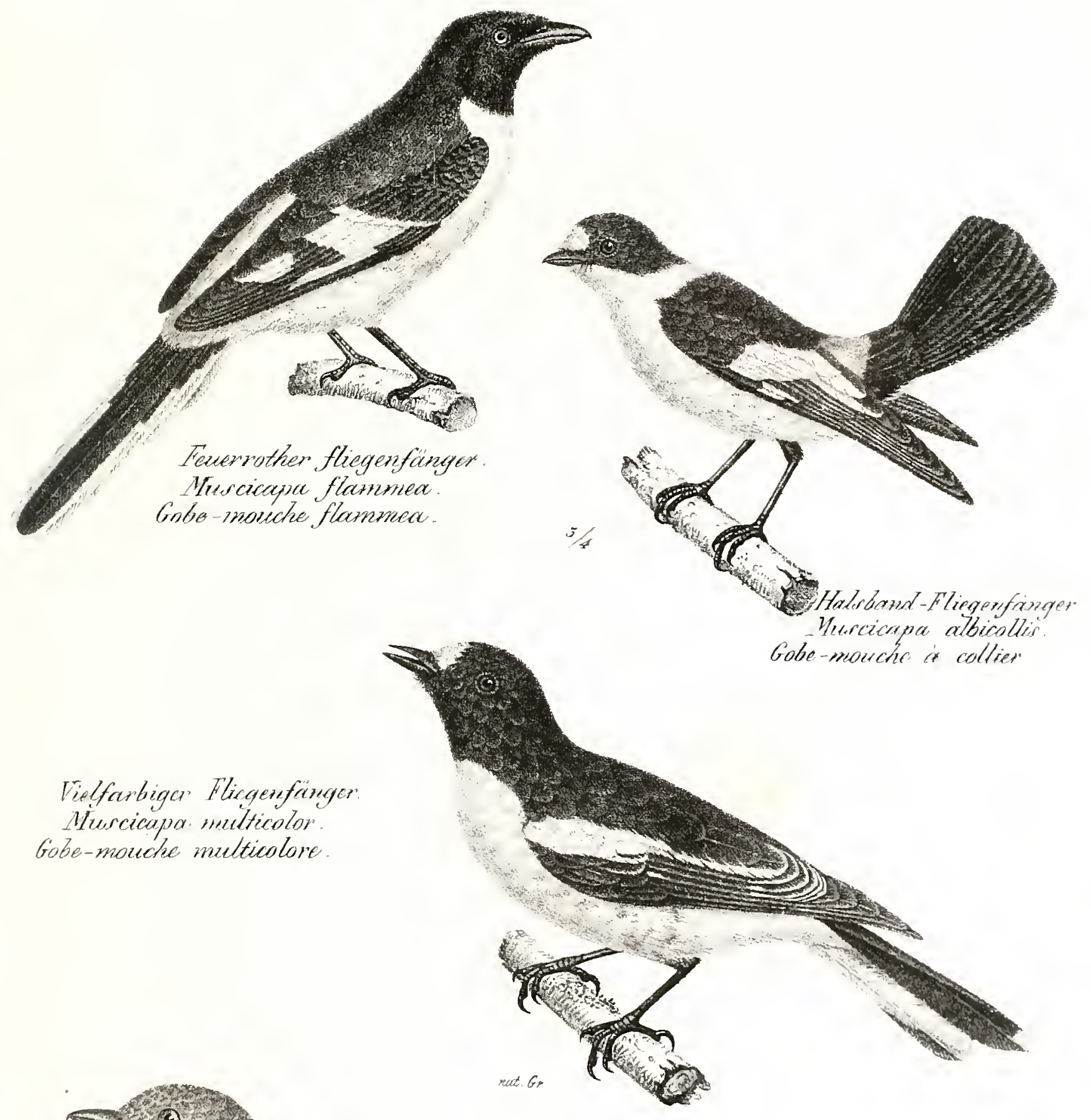

Vielfarbiger Flicanenfoungor Murcicapa multicolor. Gabe-mouche multicalore

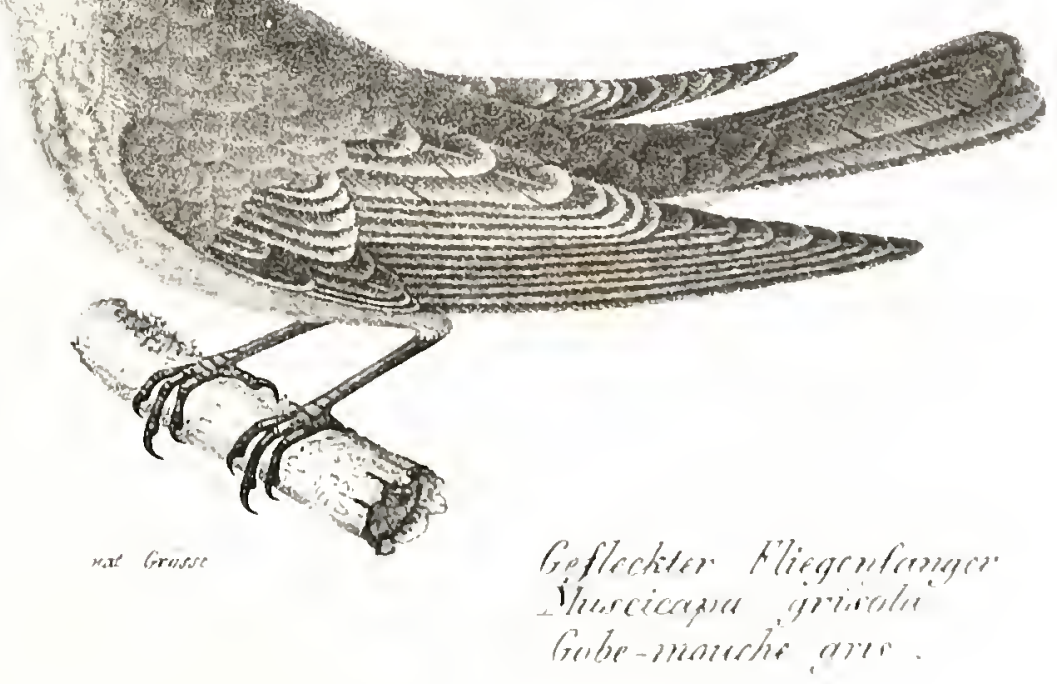





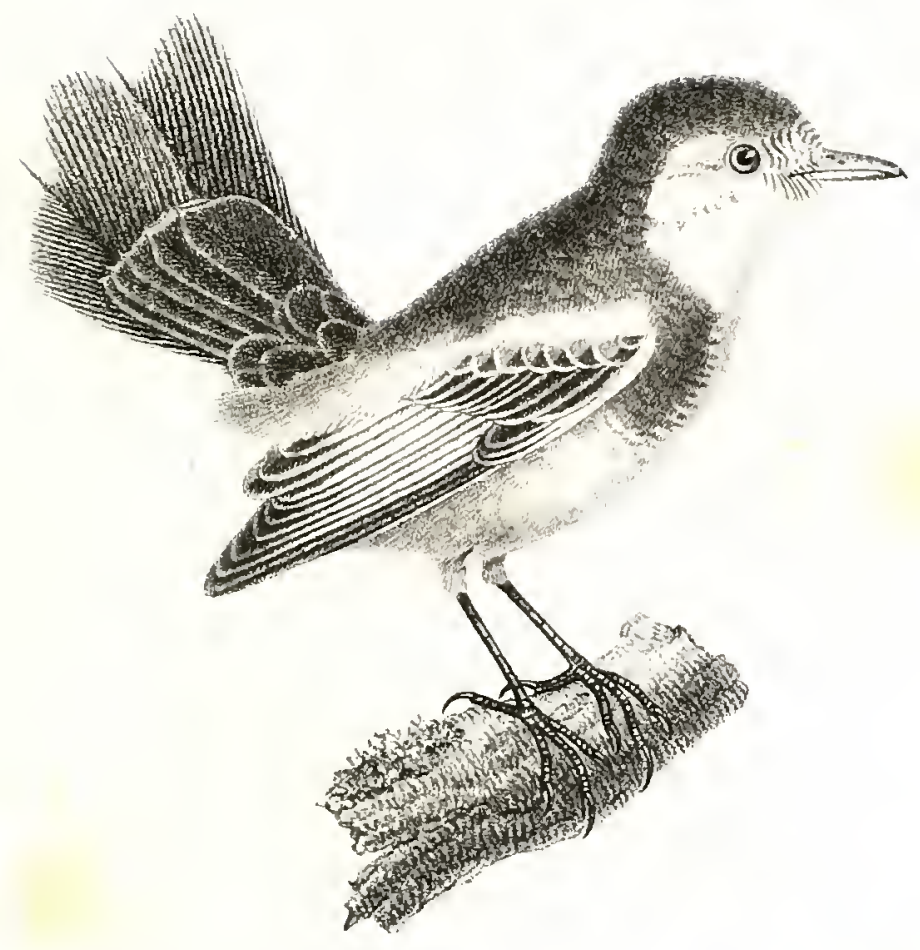

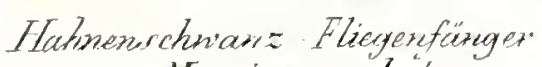
- 1ruscicapu alector Gobe-maunhe petit cog
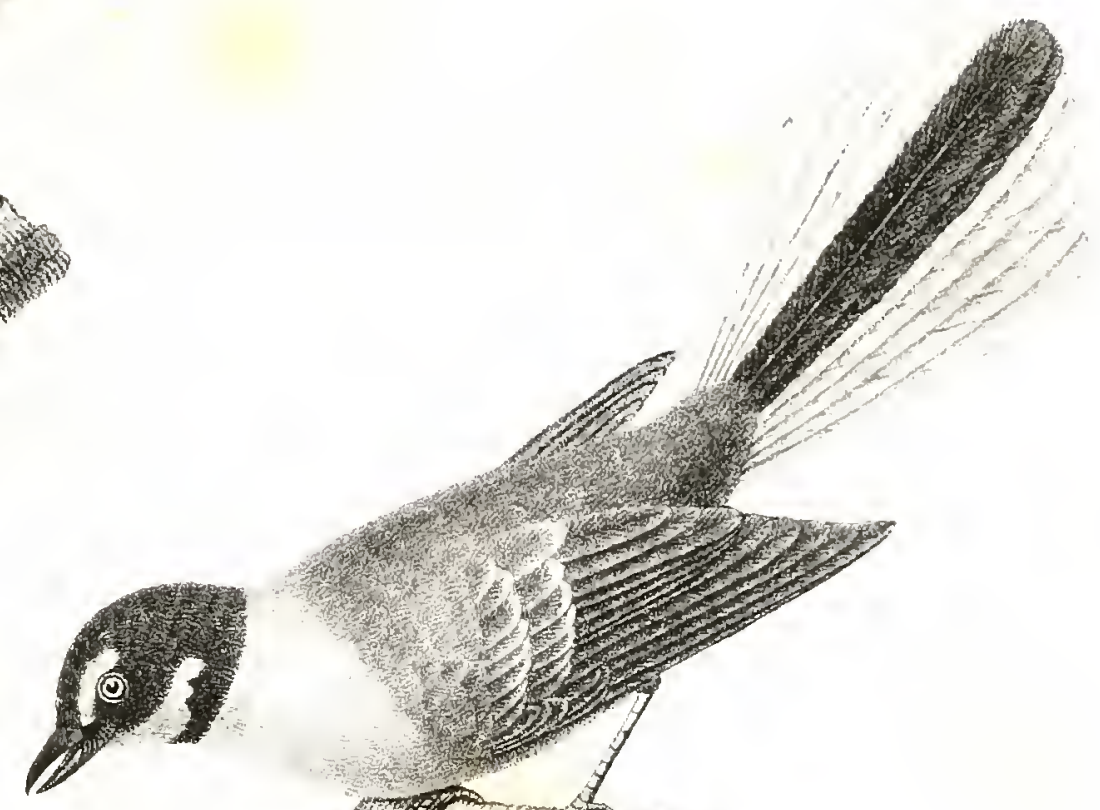

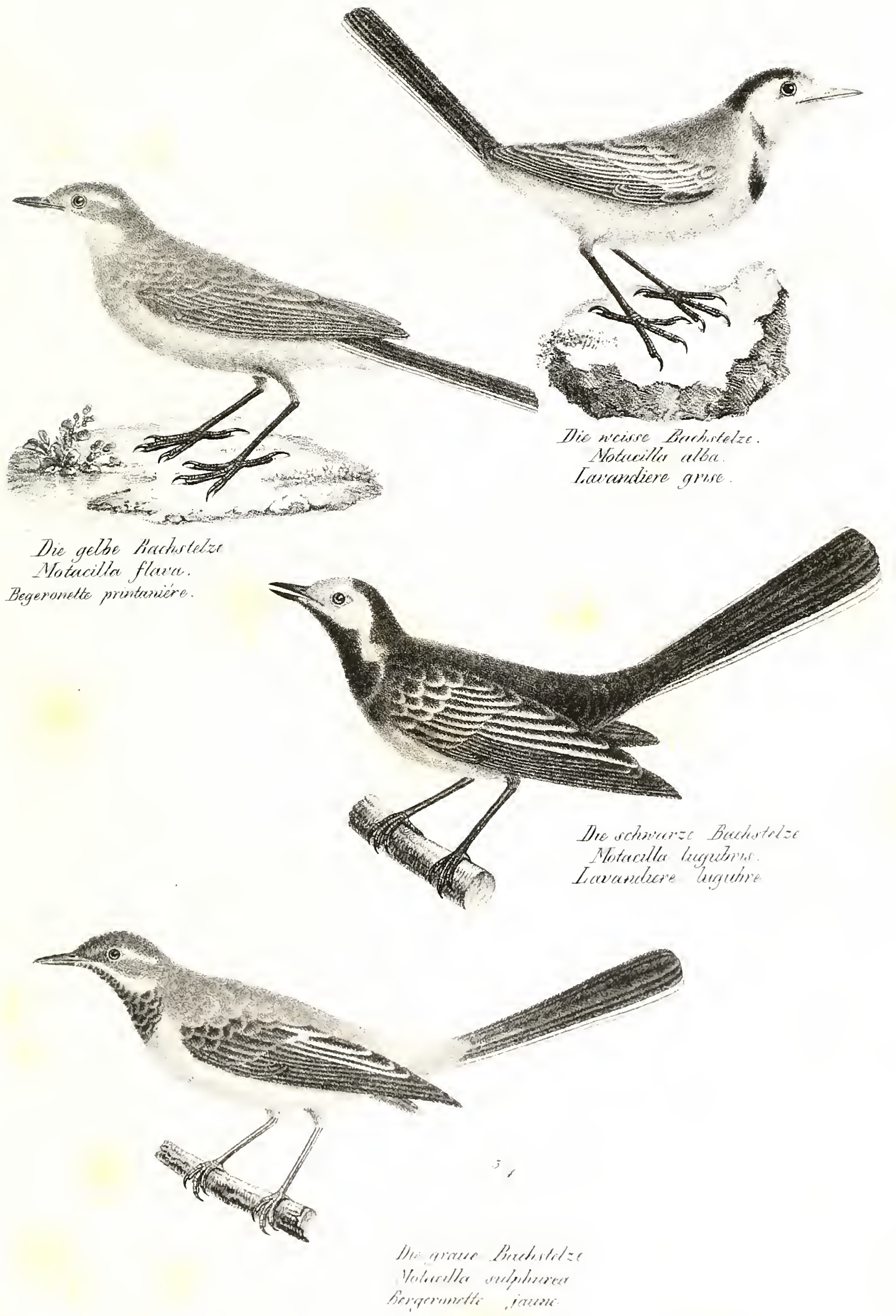

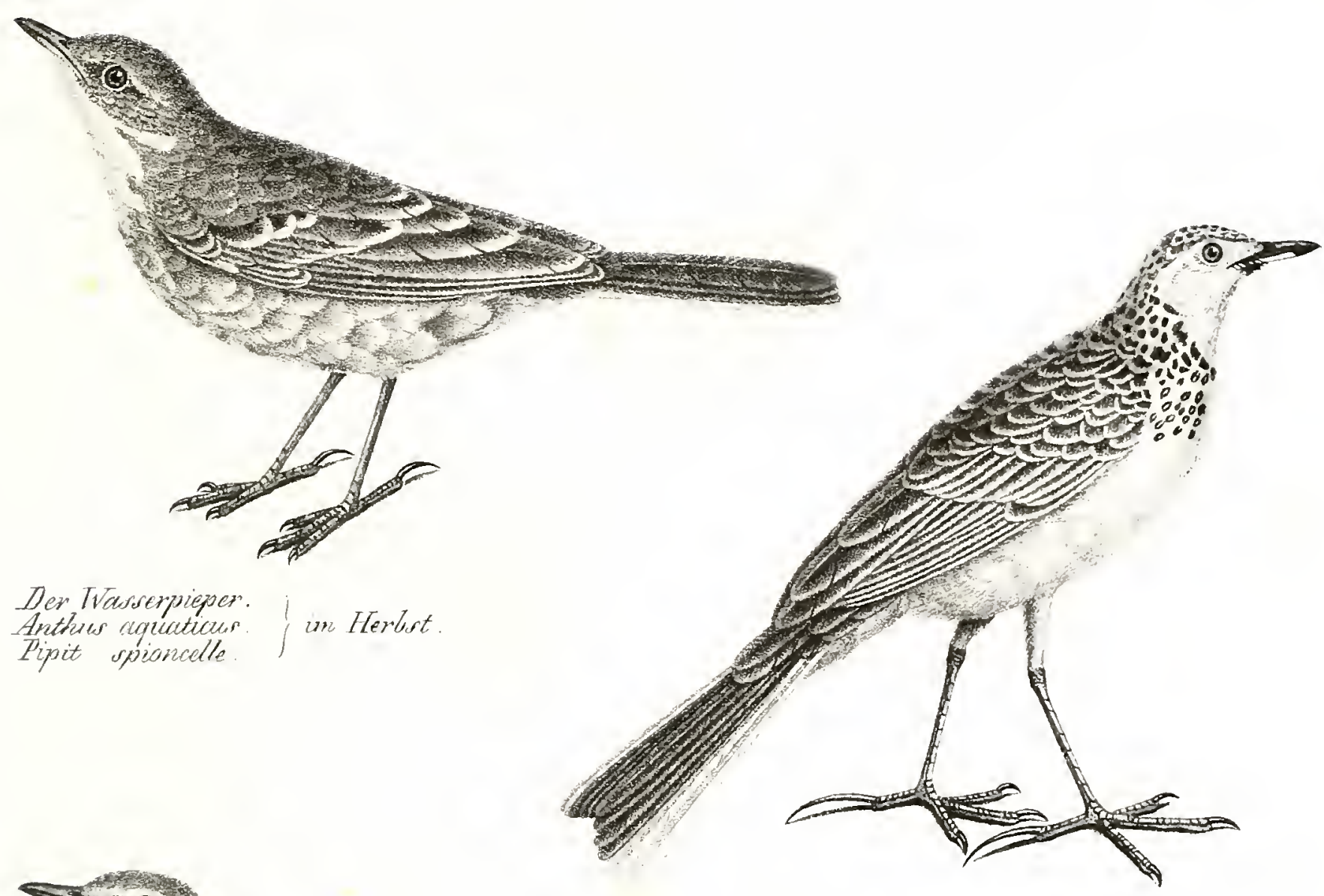

Der Taseserpiepsen. Anthas nquaticus.

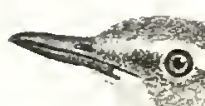

Der Richardartio Pirpes:

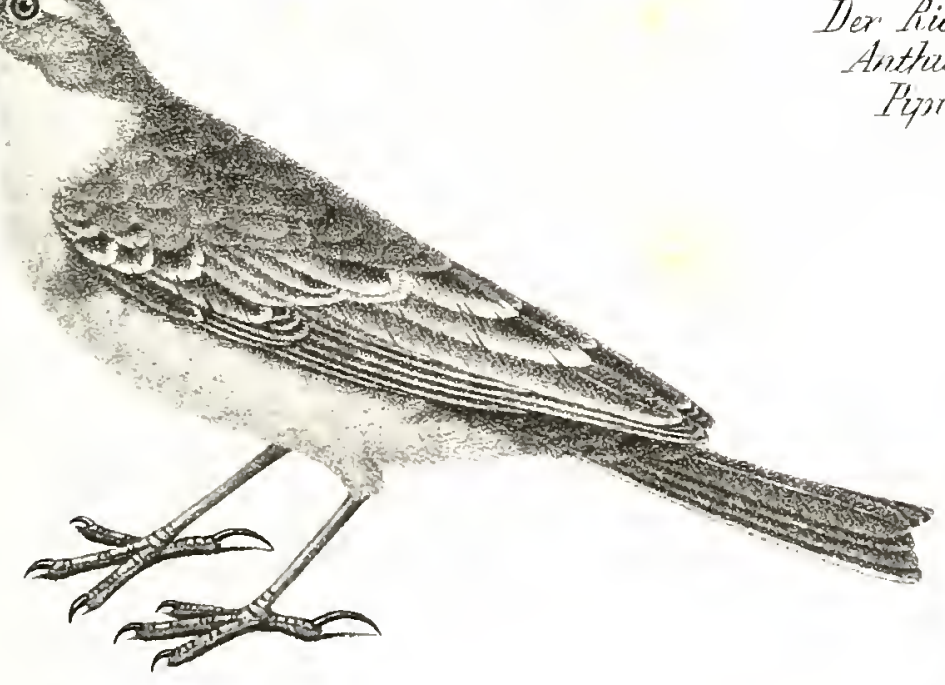

Fyprt Pichuerd.

Der thasesespricpes.

Anthes requetiones

Pipjit

spmonelle

im linethinen

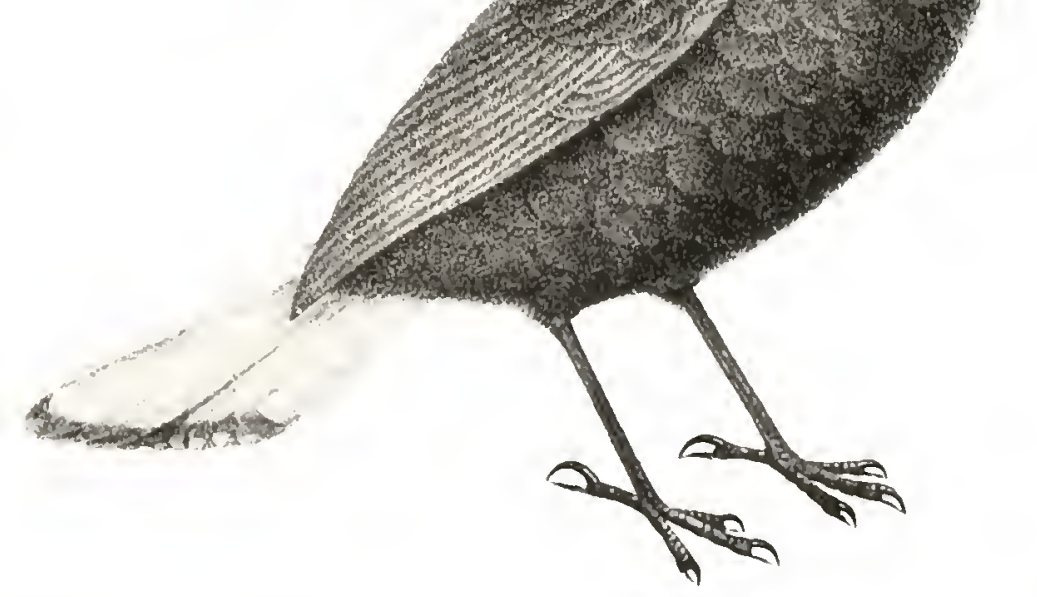

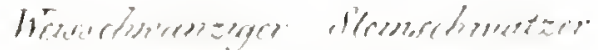

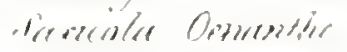

$$
\begin{aligned}
& \text { l.e cal blerem }
\end{aligned}
$$



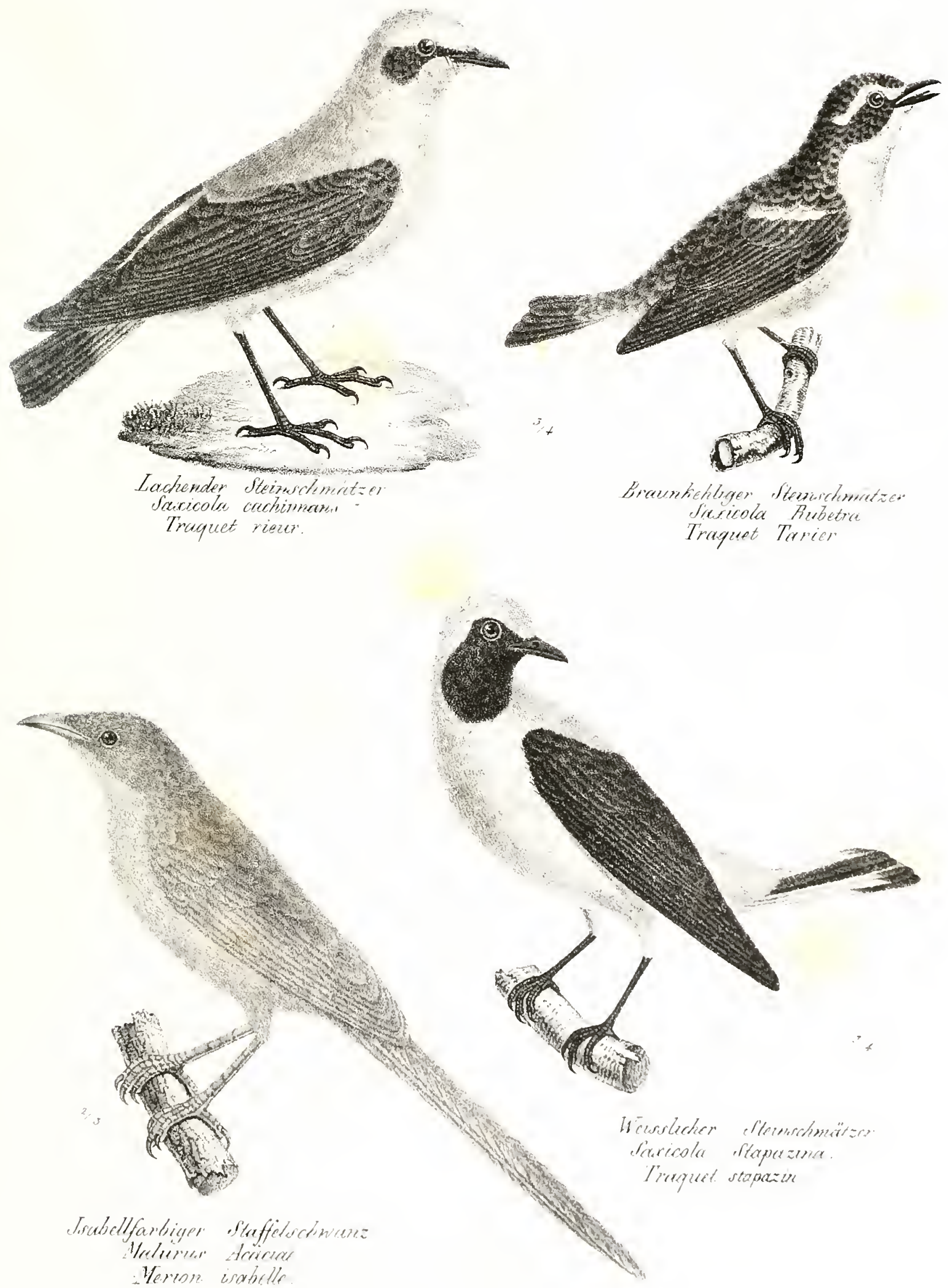


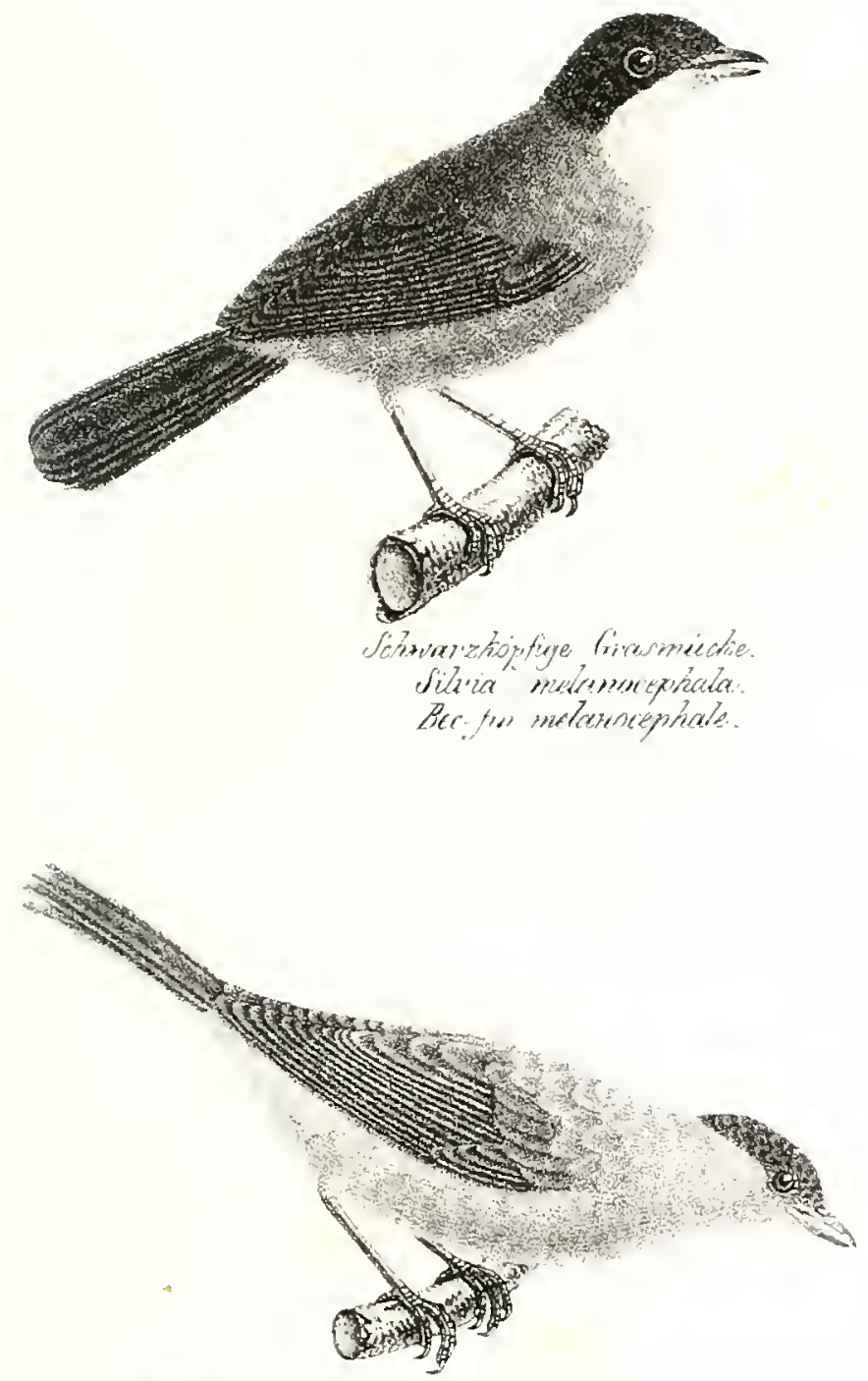

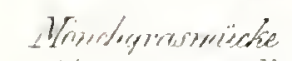
ilene uticiapula Bece fin a colotito min.

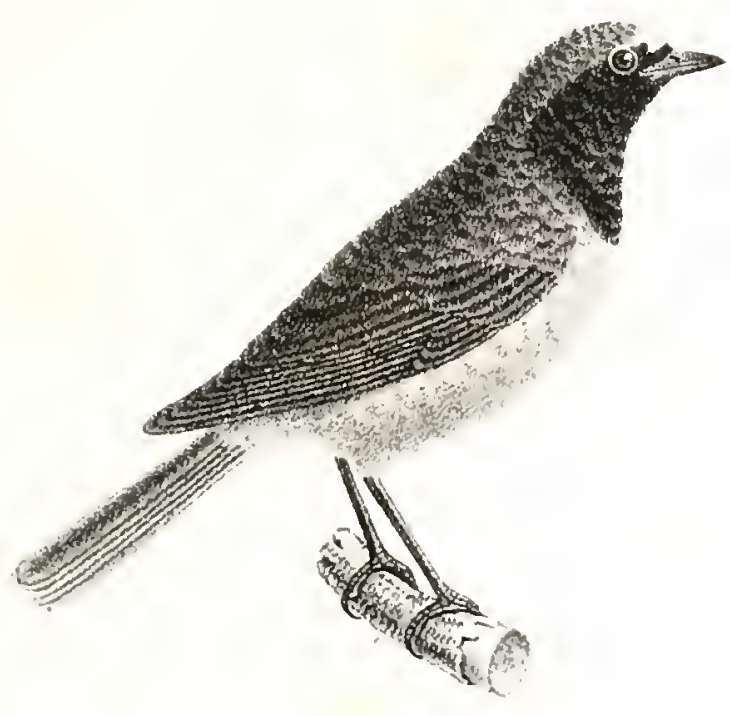

frimtan-fintherna.

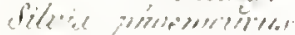

Des fin its merrastles.

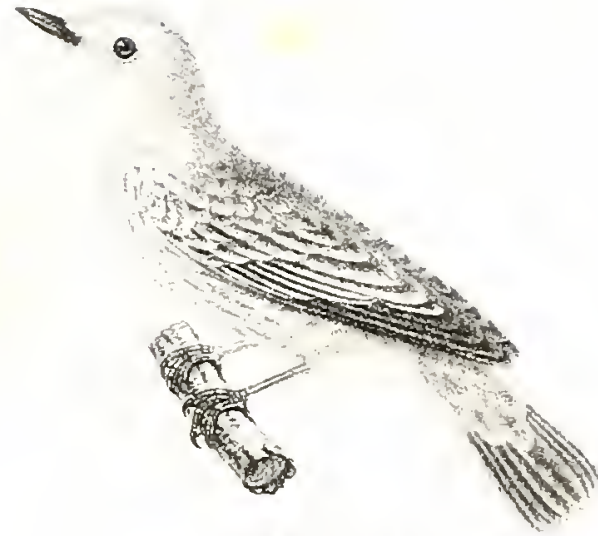

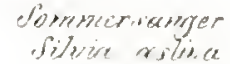
Bes-firs riteing

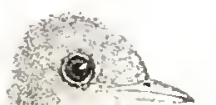

Gavterlacilereque Sulece mypntiris.

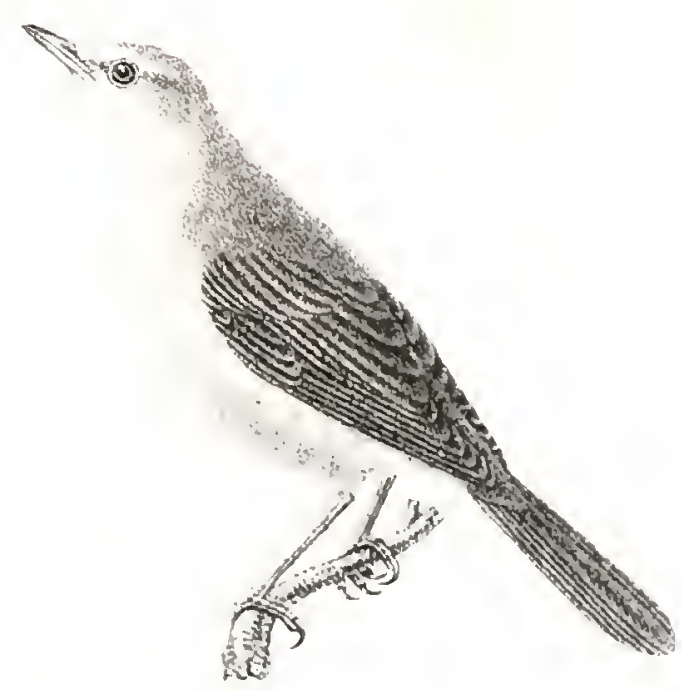




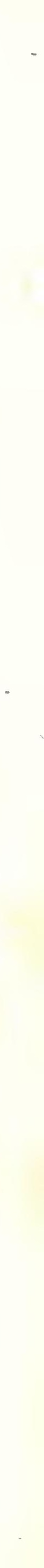



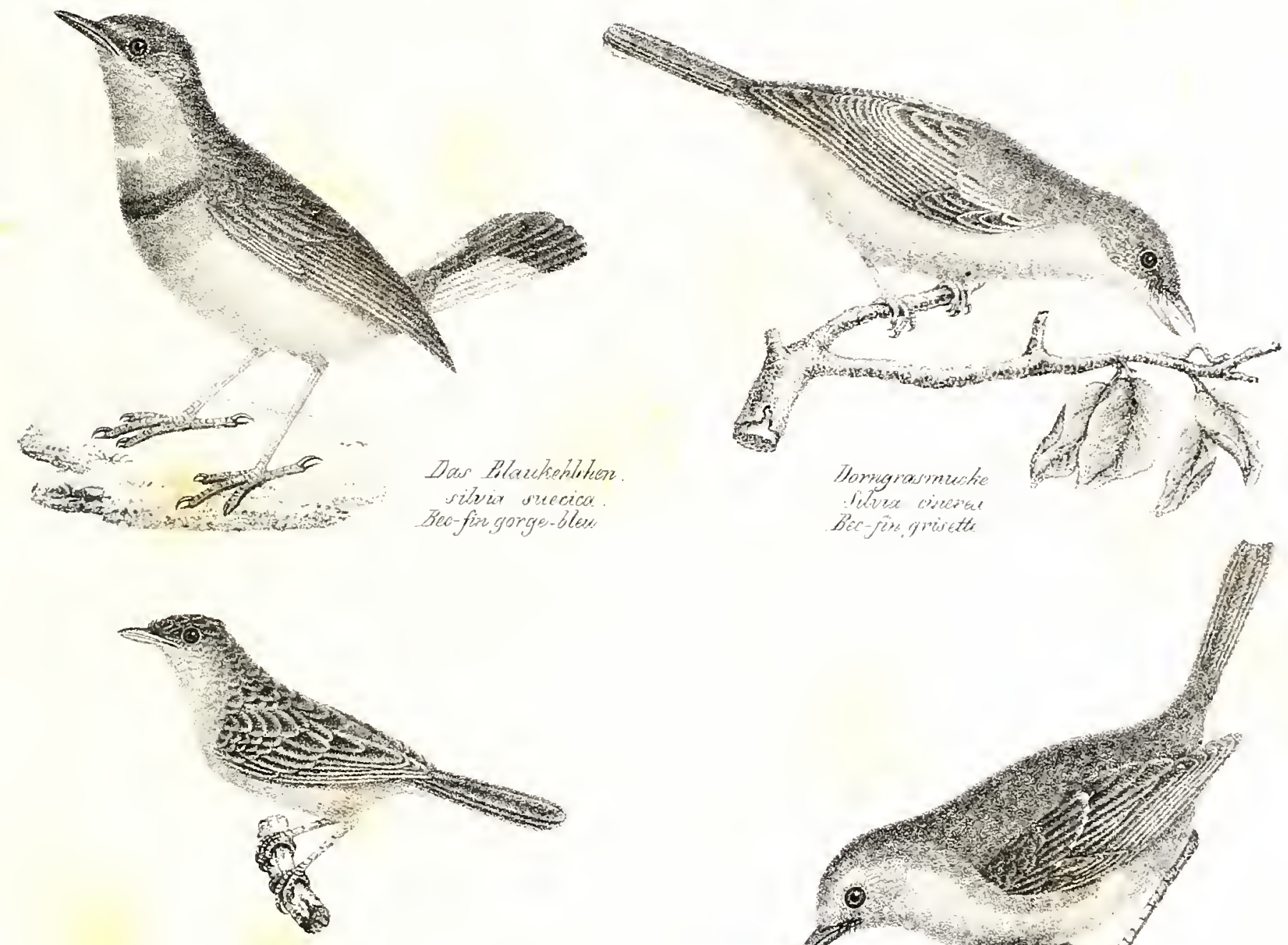

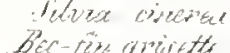

Cistensianerer.

Sitrix. cisticulas.

Bec-fin cisticole.

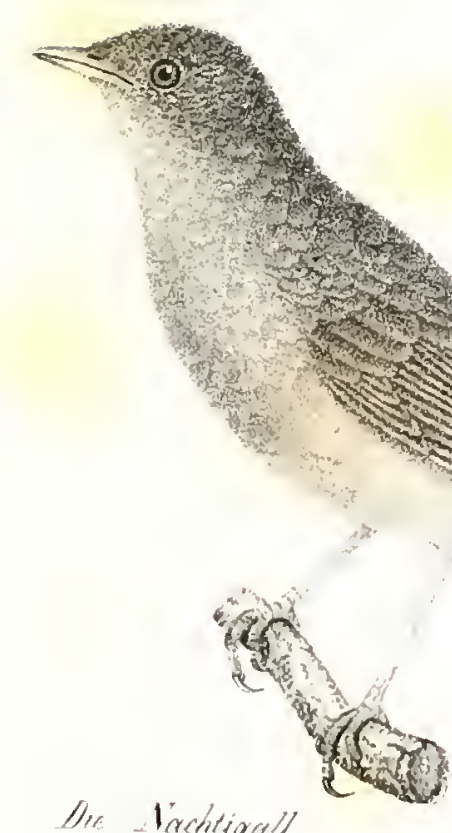

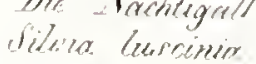

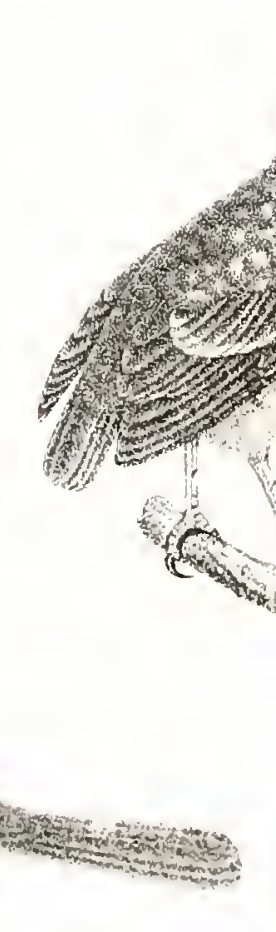

te Riosirignal.
Not

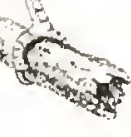

Roththchichen, - ferreger

Silewe viberister.

Ber fin rouga-gurigo

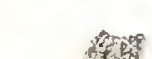



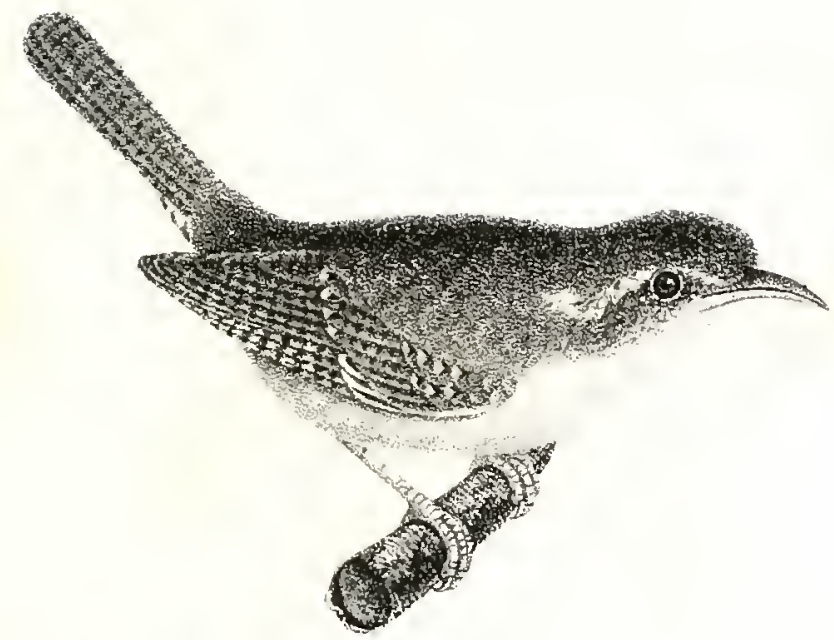

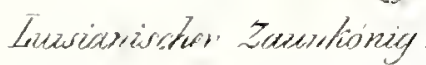
Trogloditas Tudavicianus. Truglodite de la Louisinue

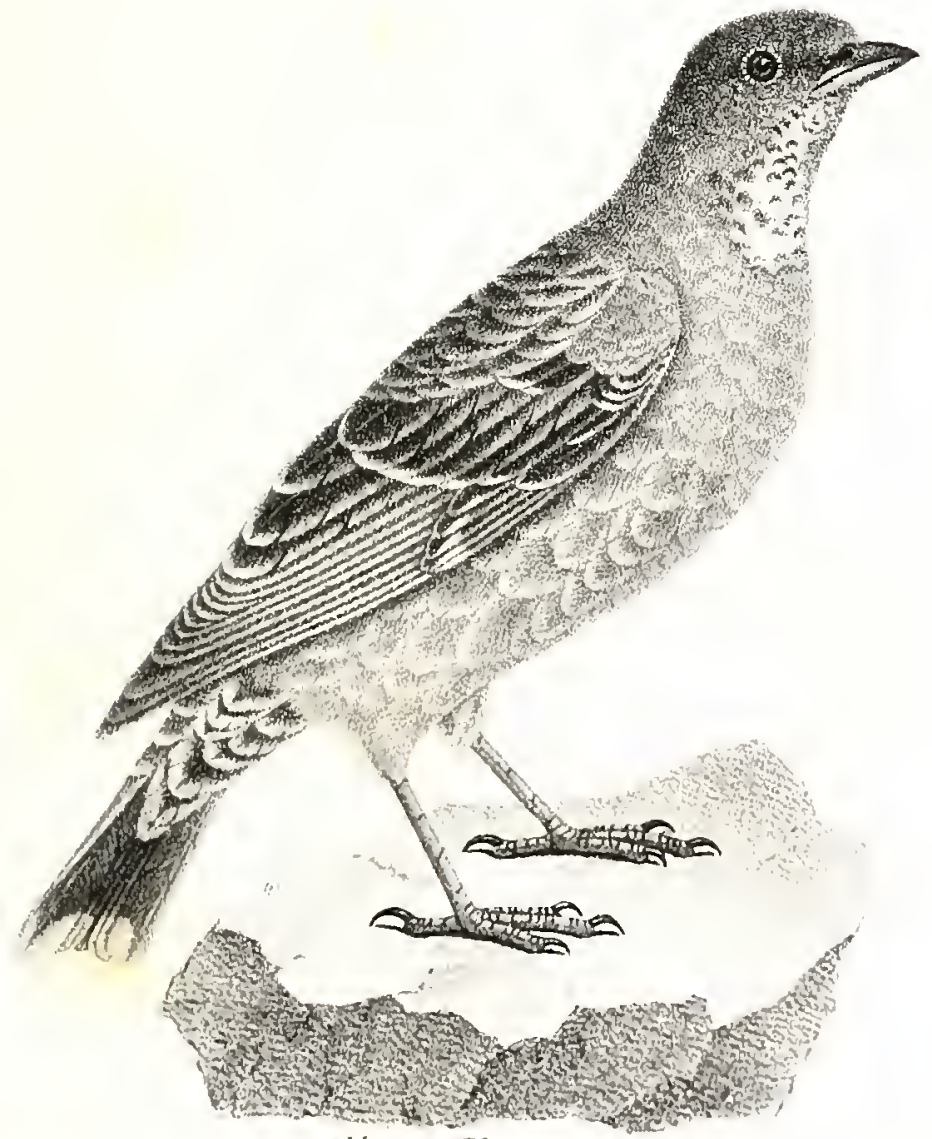

Alpon- Mlicenogol

he leygot der ellower

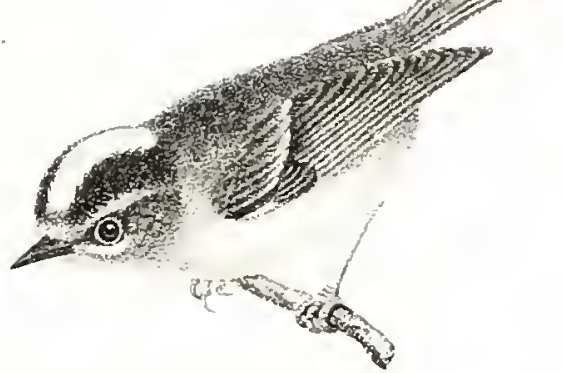

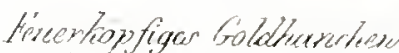

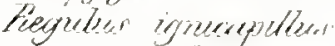

Biorteles tripule brendorme
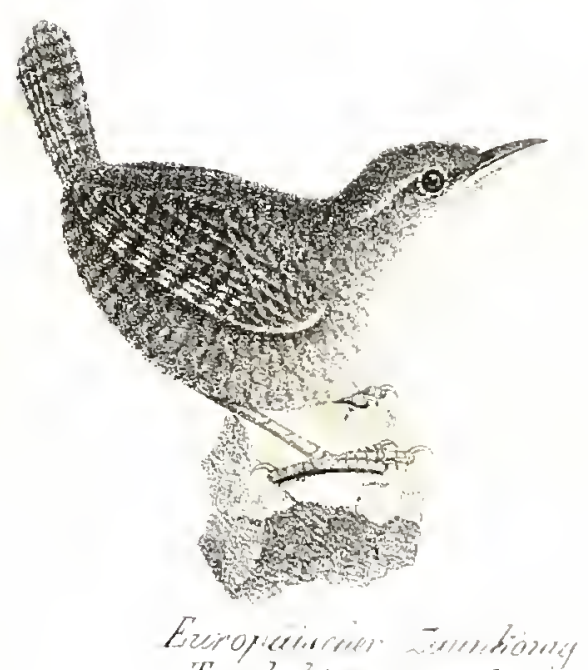

Troglocletes parrwhas. tie Troglodyts

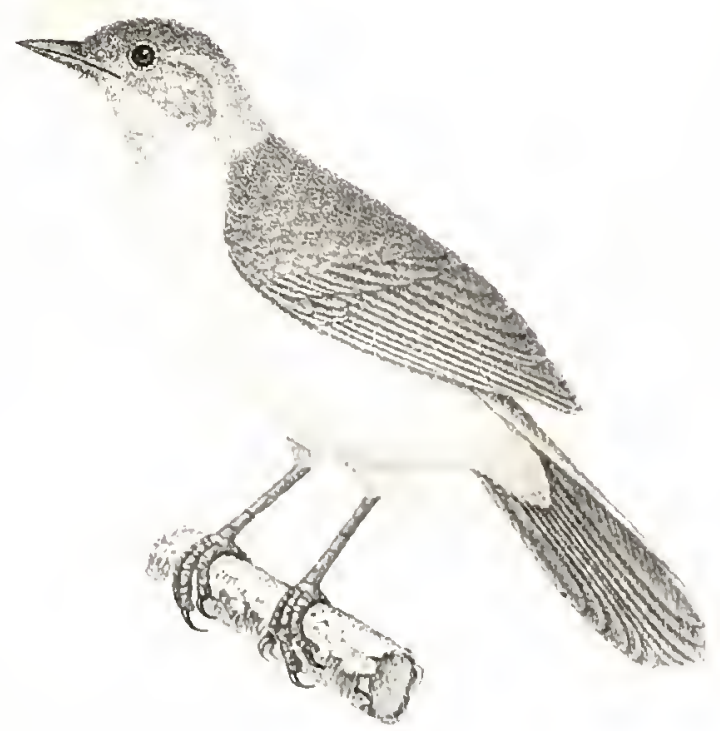

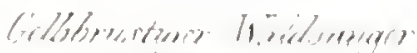

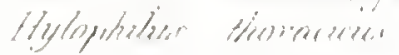

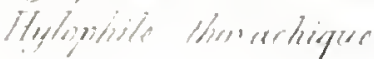





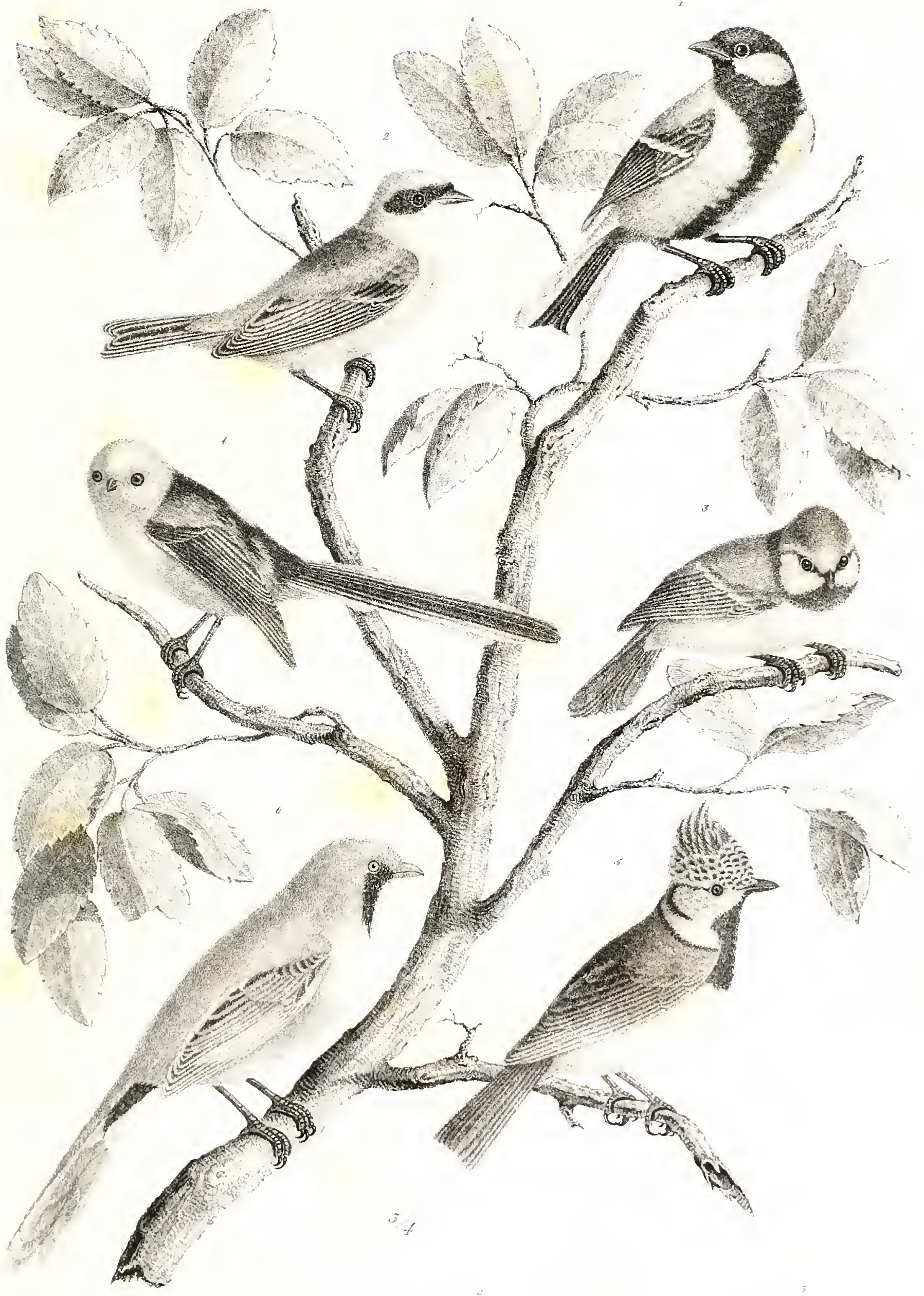

\begin{tabular}{|c|c|}
\hline 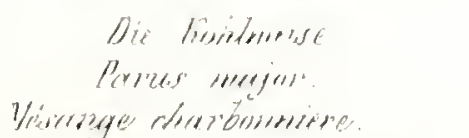 & 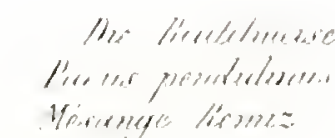 \\
\hline 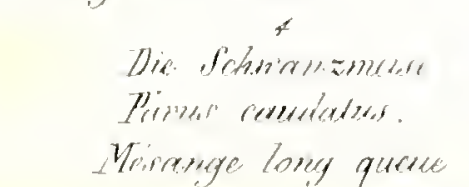 & 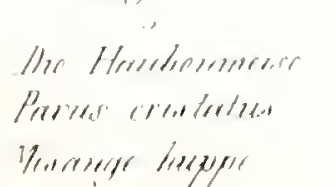 \\
\hline
\end{tabular}



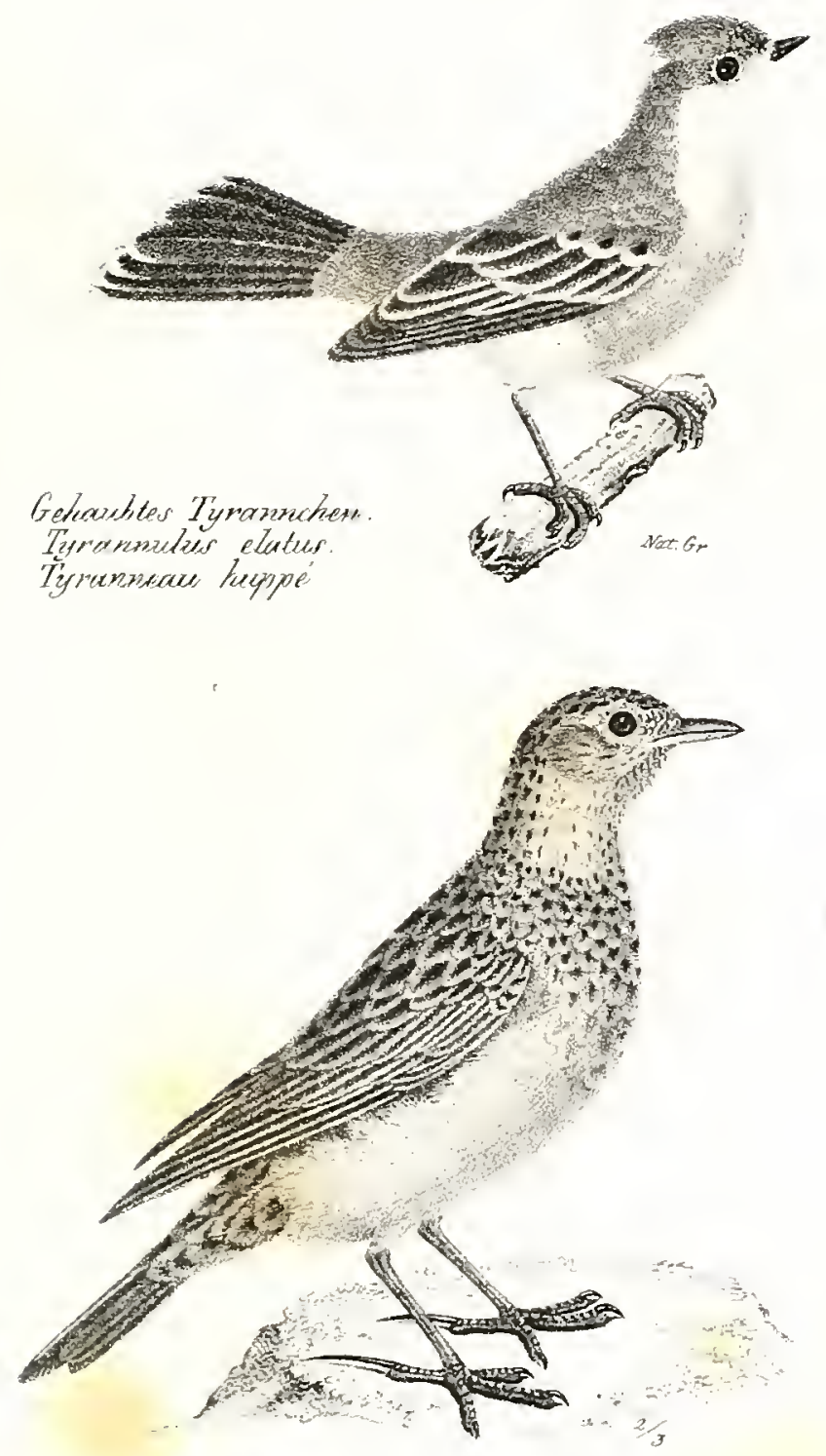

Fidllerctio

Atousced serverses

\section{Alouette ovelinsirn}
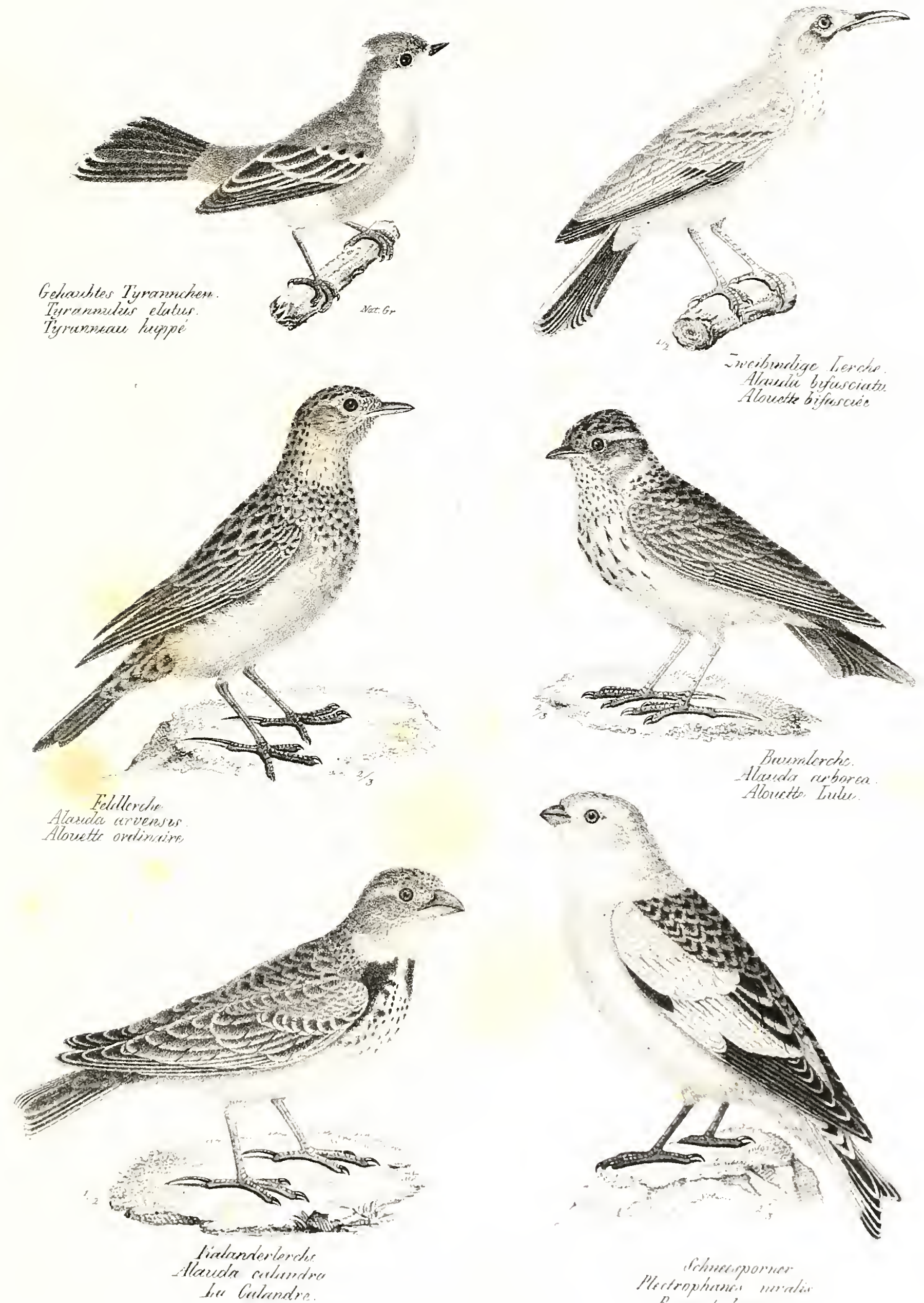

$\Leftrightarrow 0$.
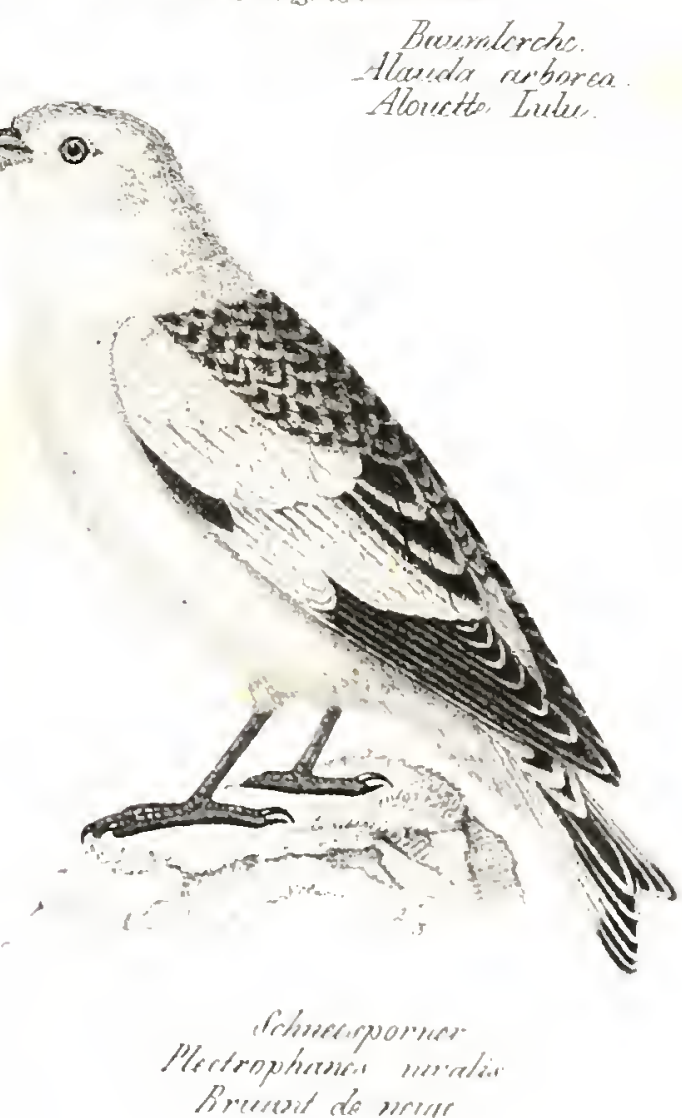

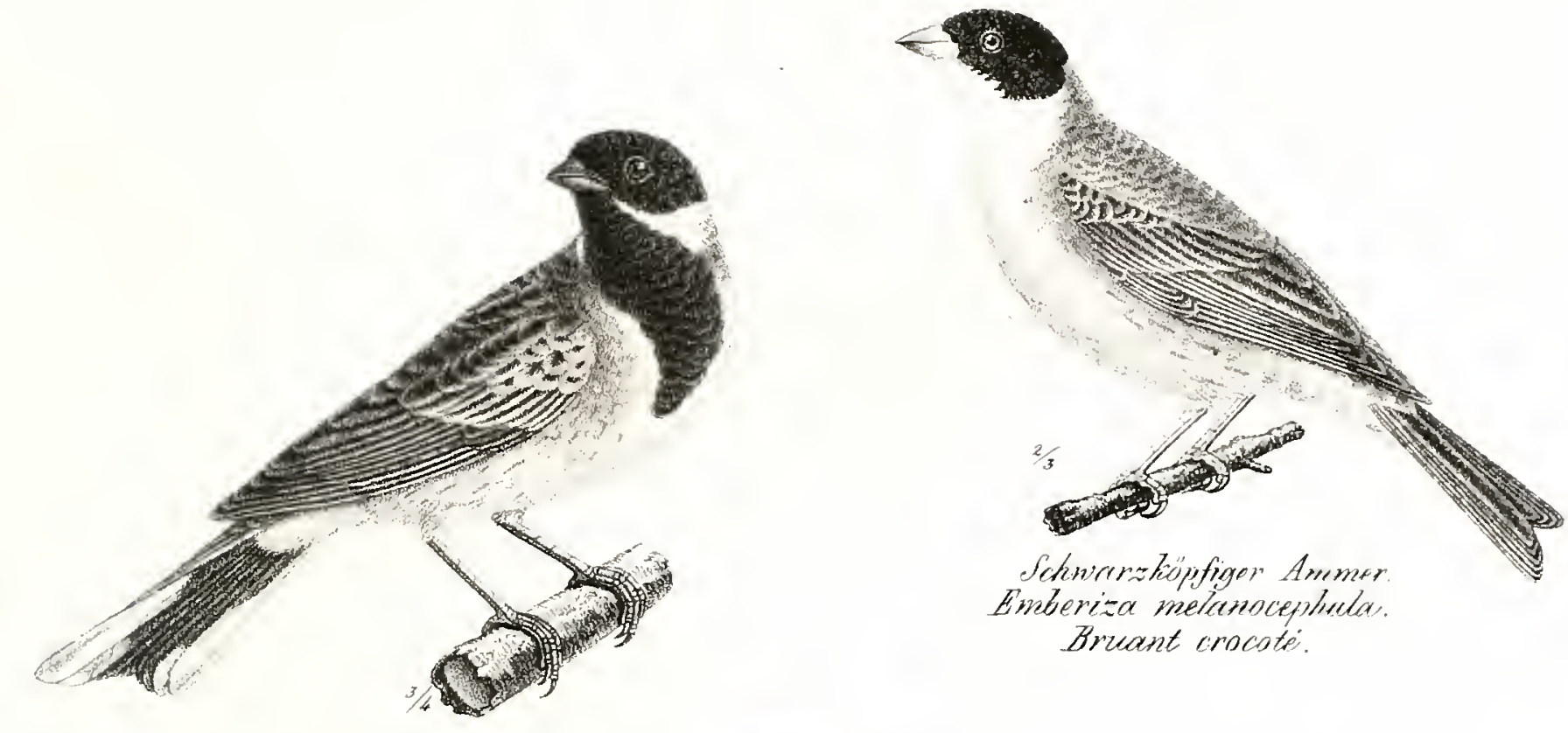
Aimberiza melicnocepliula. Brwant erocots.

Piohr-Ammer.

Emberize Schoeniches.

Brusent de raseanow.

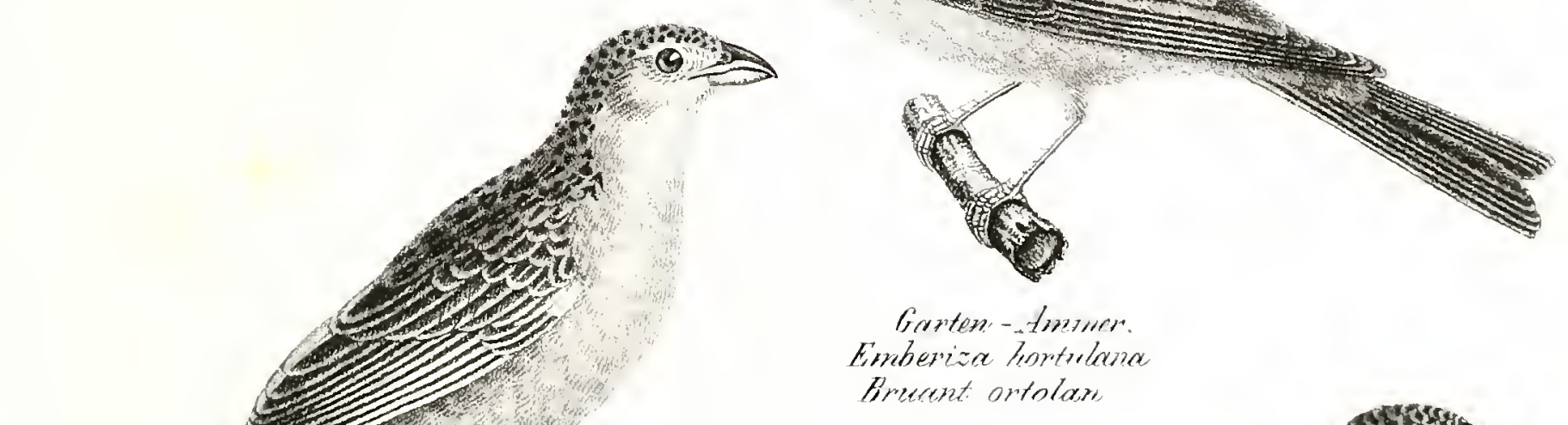

Granten:-tmanes.

Emberize hortulene

Bimerent ortolar.
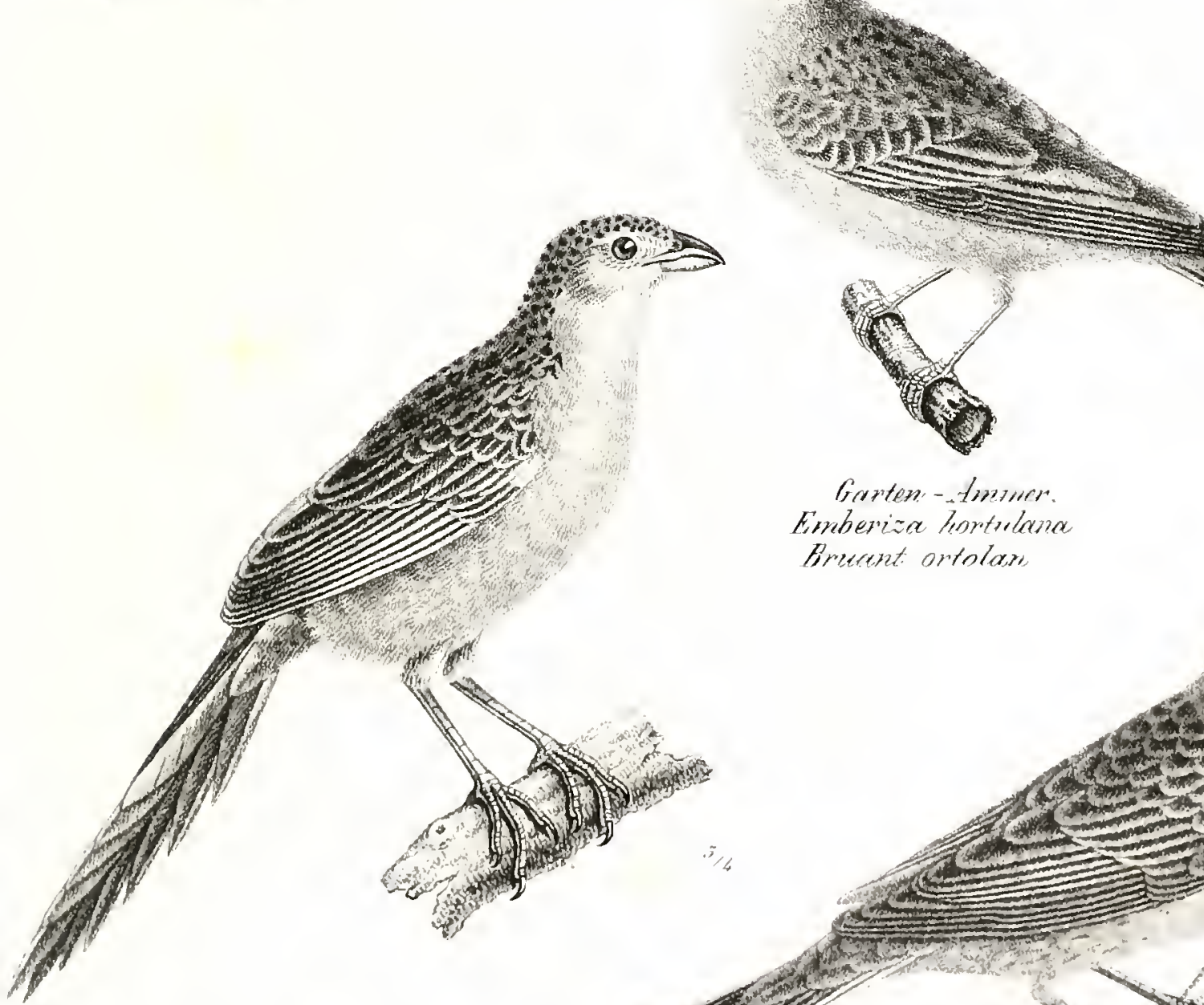

Gerandetos Amonerlinas.

Emborizaides minrginalus. Rimberizoids langibando.

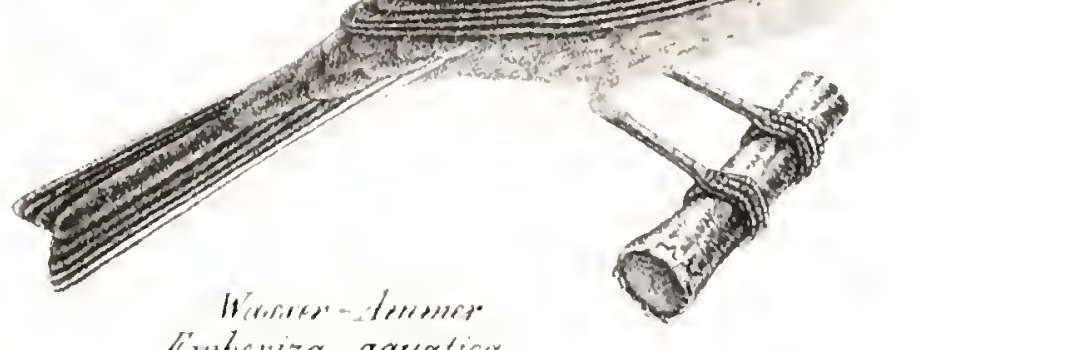

Cimberiza aquatica

Brueant de morrent 



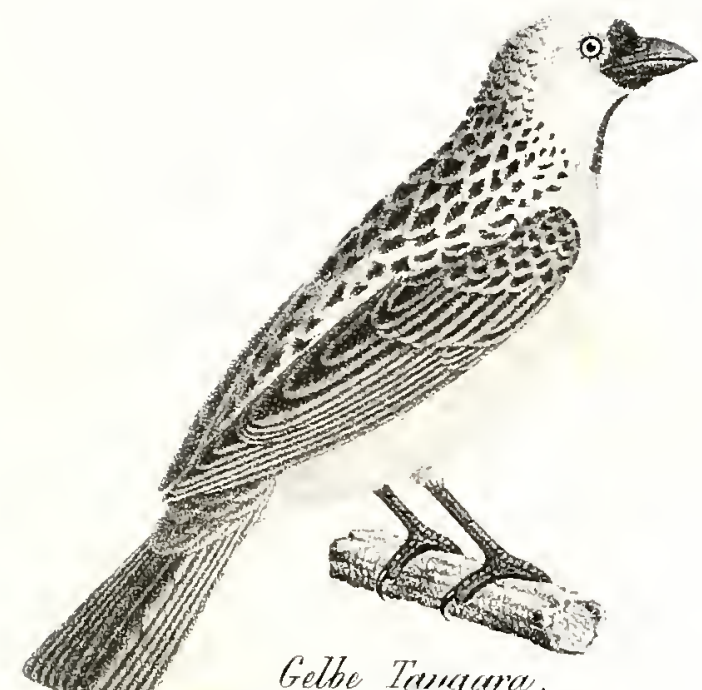

Gelbe Tenugerra.

Tenagrne citrinelles

Tangara citrin.

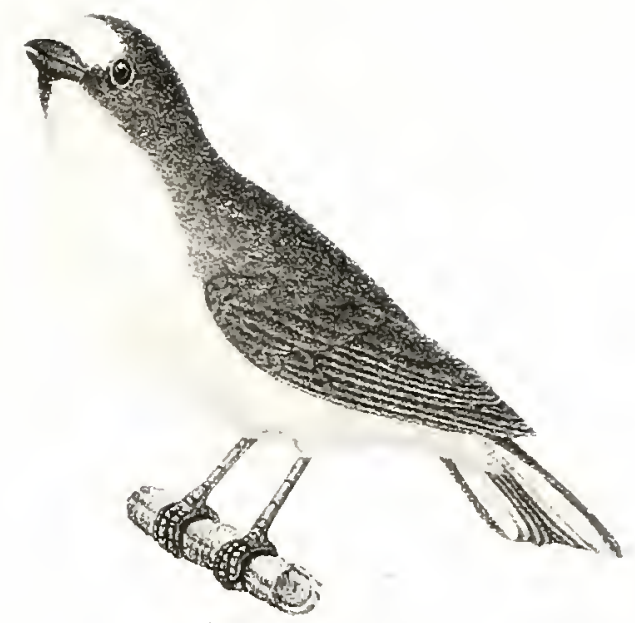

vulette Tanagera.

Tasagra vinlacen.

Exphone tcite
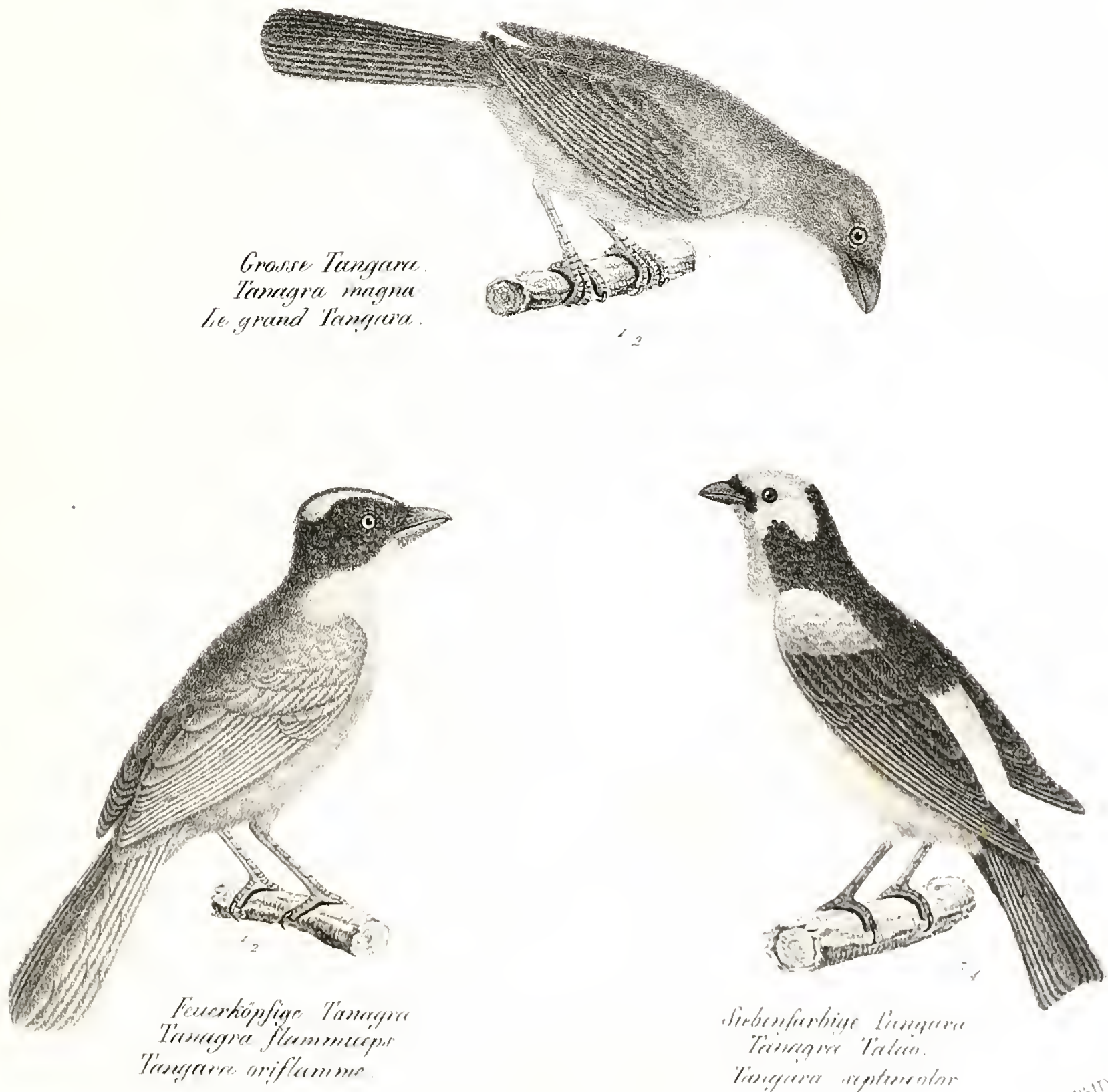

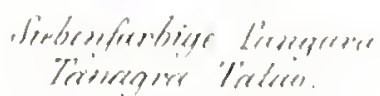

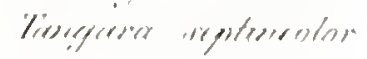



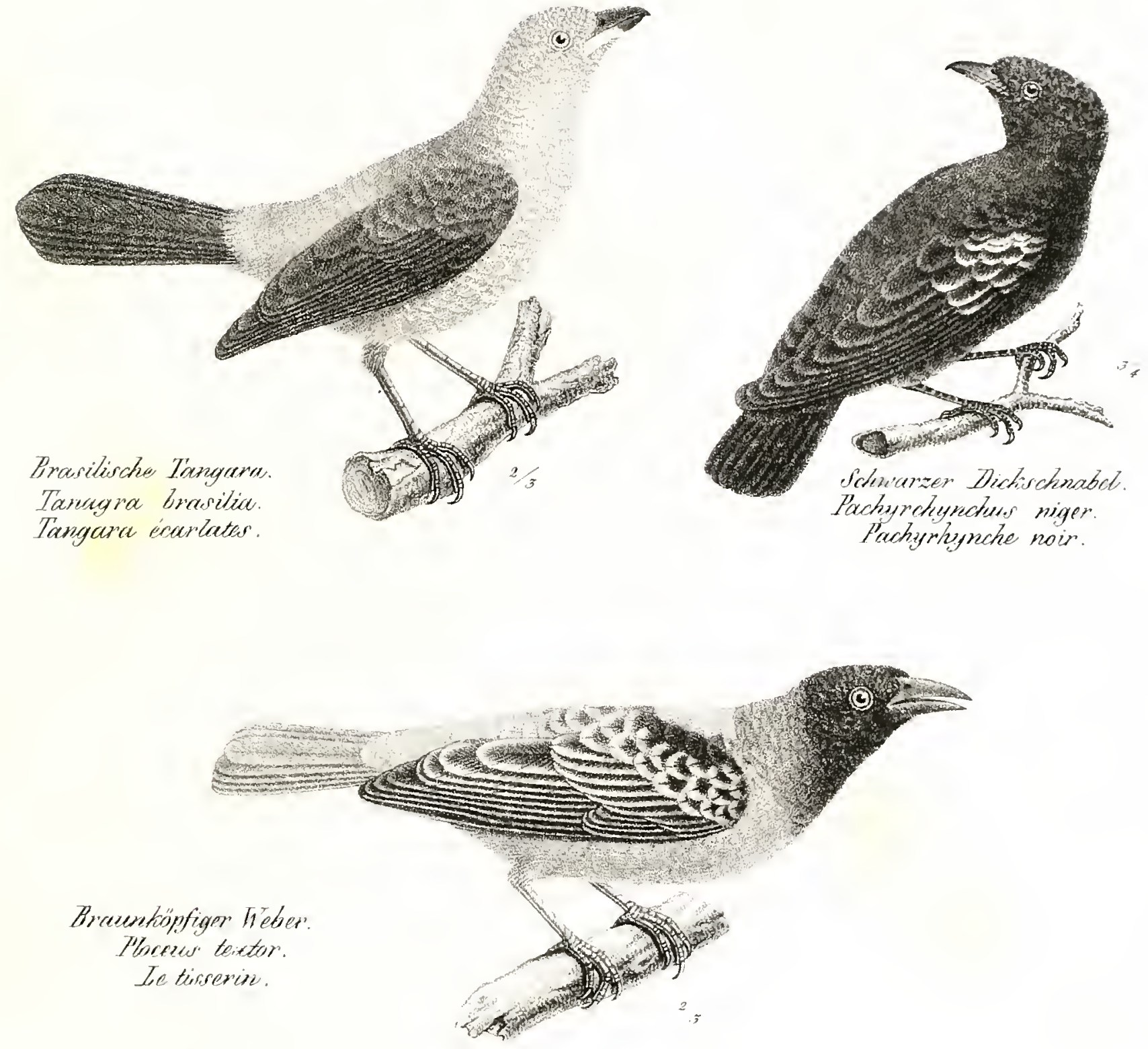

Brecuntionfiger tieber. Plorrew textor. lie tirserian.
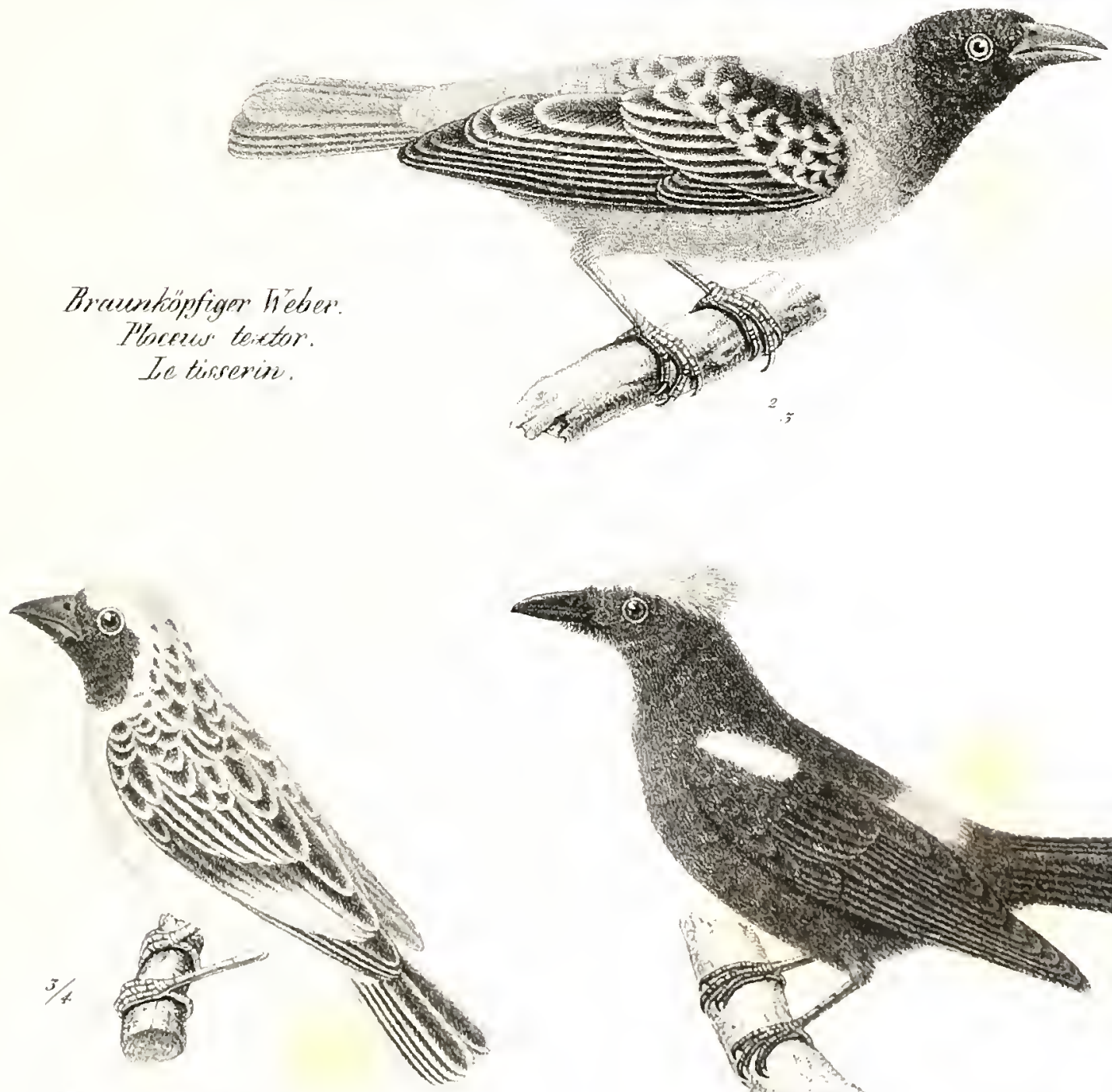

Der Tutinsentioseric. Plocenes philesprisueno

Tucruam - ormentei

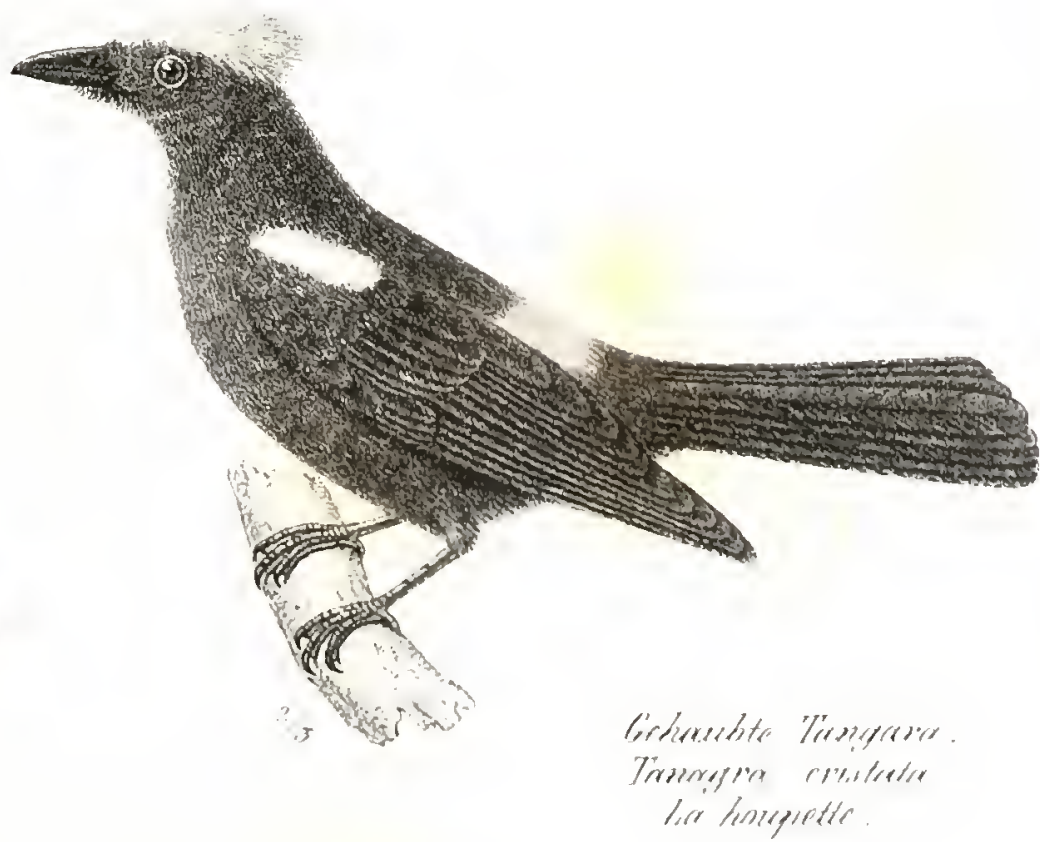


Der Pivischthermbeisser
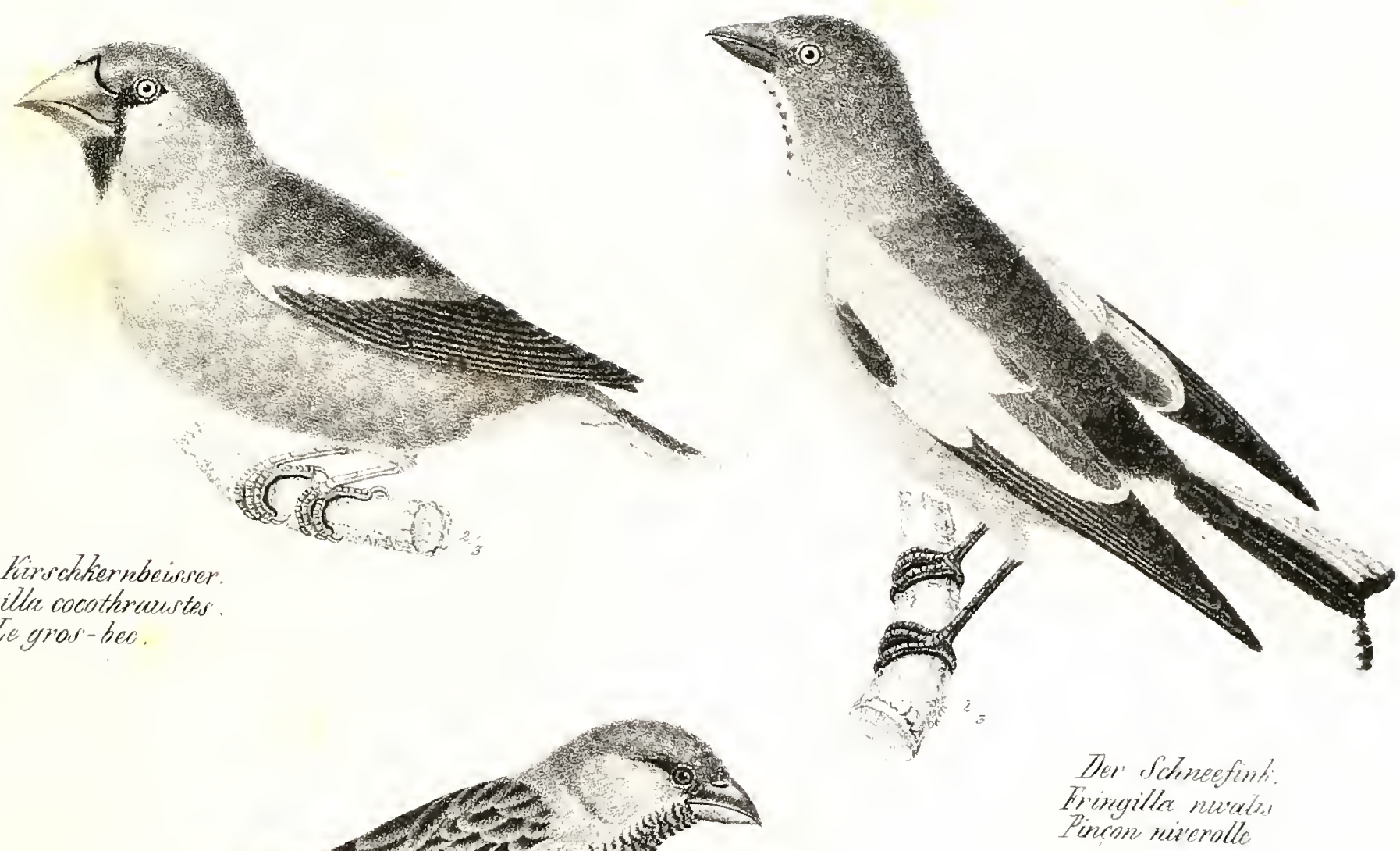

Der Howessperling

Fringilla dorrestica.

Ze maisean.
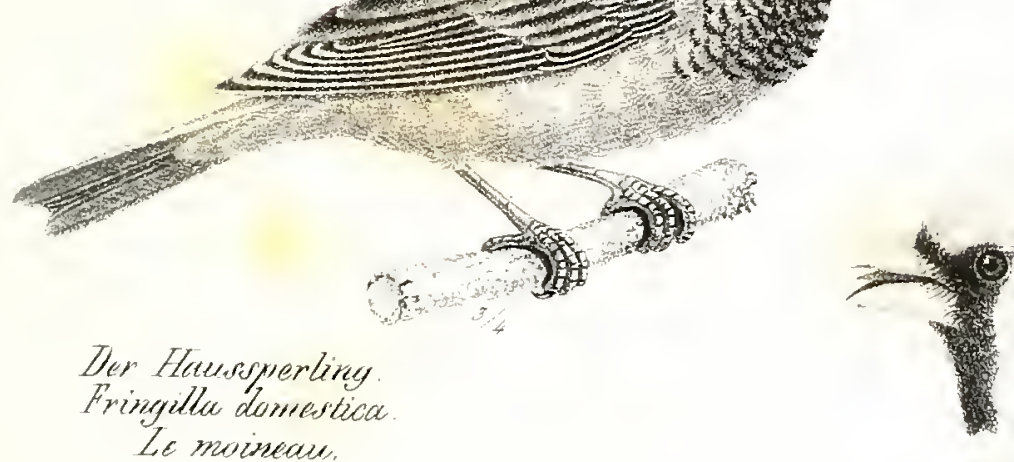

$122^{2}-20$, w

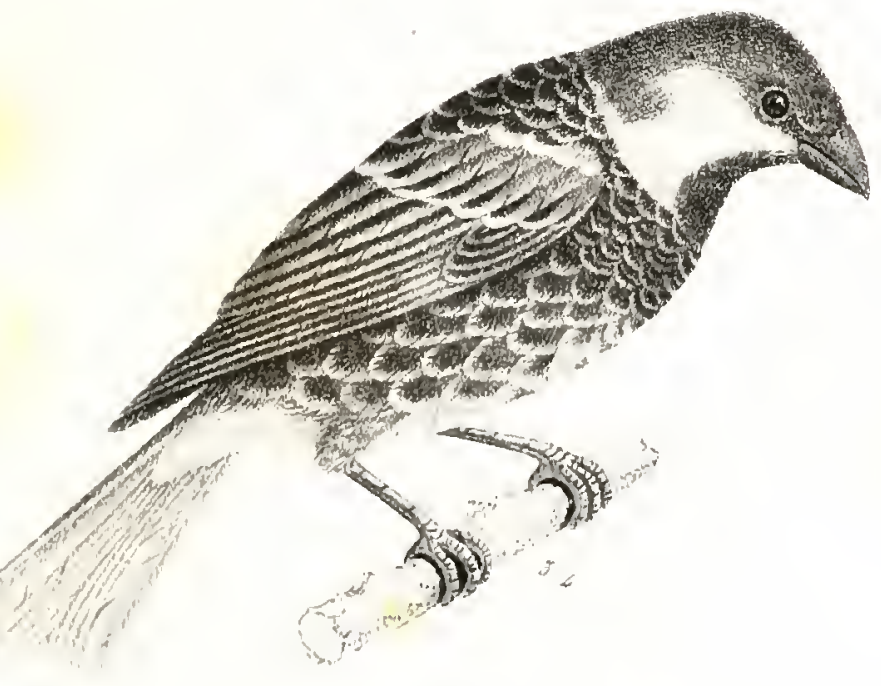
Der apecencioctios dyentisus

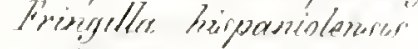
¿Yomencue éspragual.




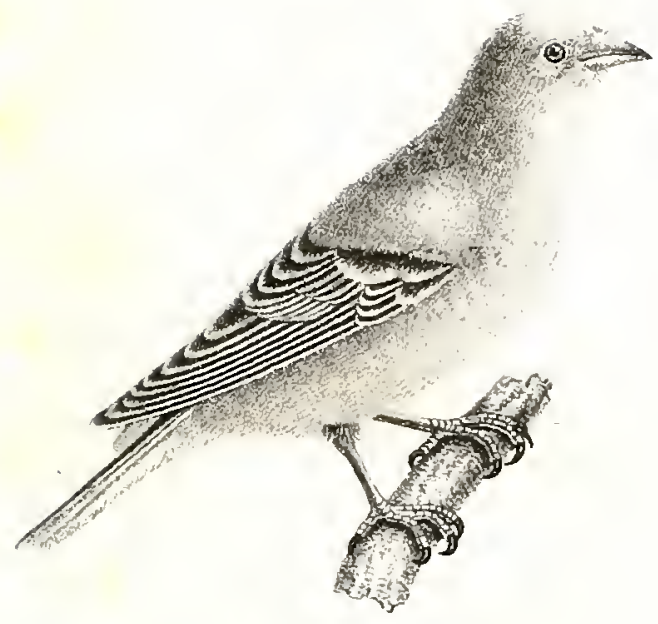
Der Eitronzeirig. Le verturom.

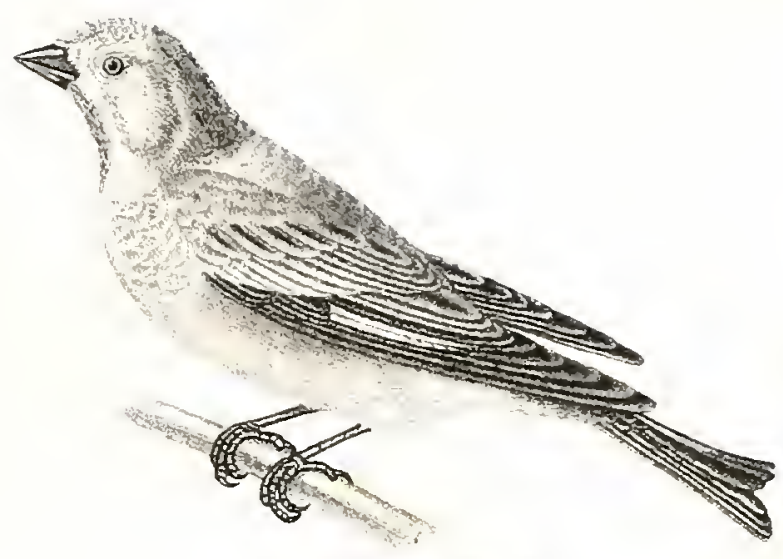

Her Bilethanfliong Fringille connabina tia grend Linate.
Der Bergfinte

Frimgilla montefringilla.

Gros-bec d'Ardenzes

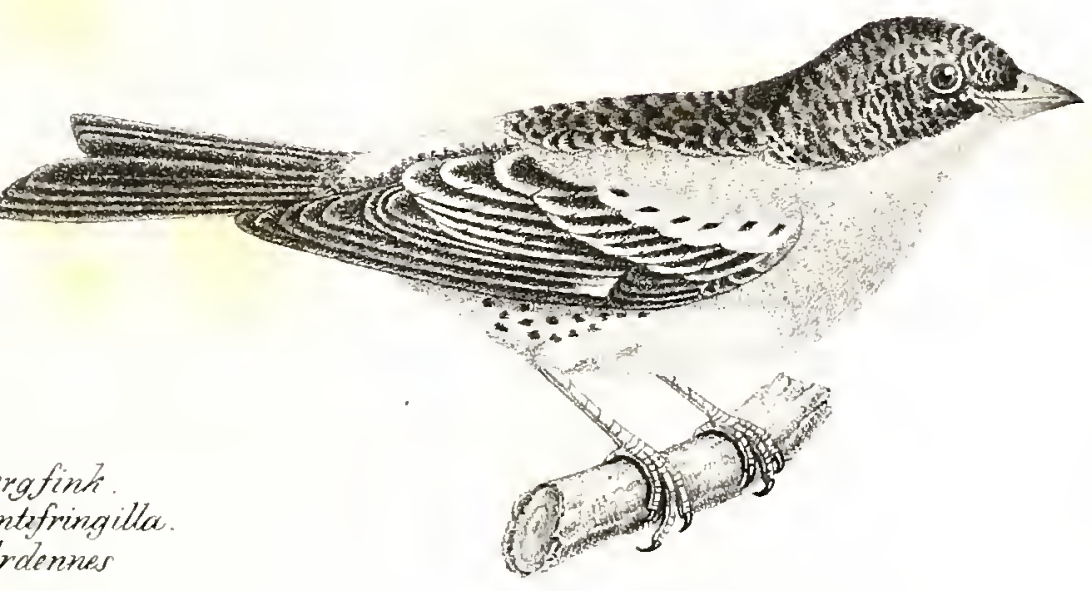

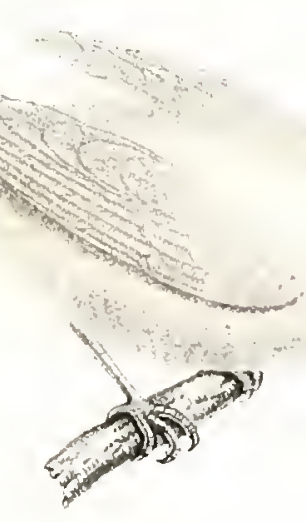

Der Gunarienzedirig

Frisgilla cumaris - feriz de casinrier. (c)

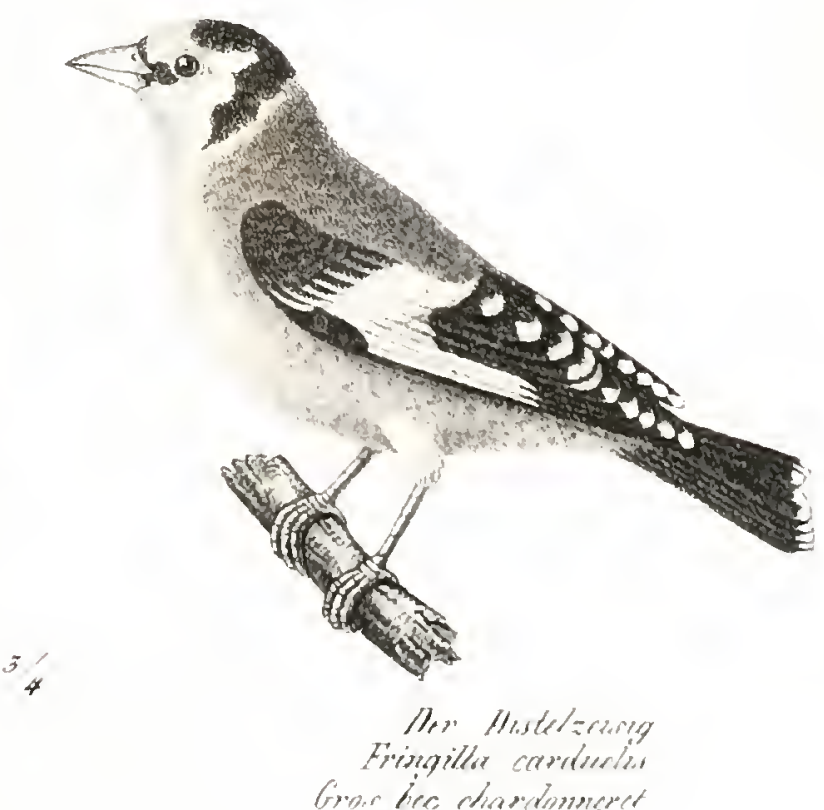




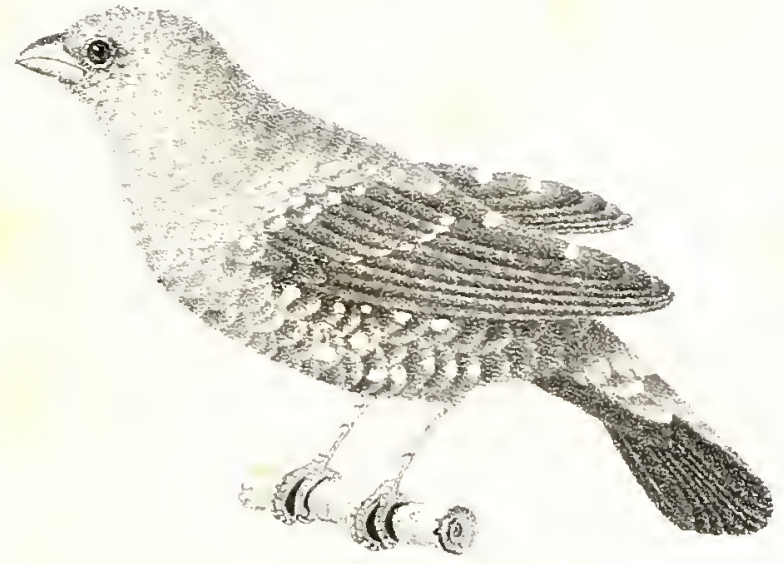

Geterpfter. Berigedi:

Finingille strecuidern.

Bengoli piqueté
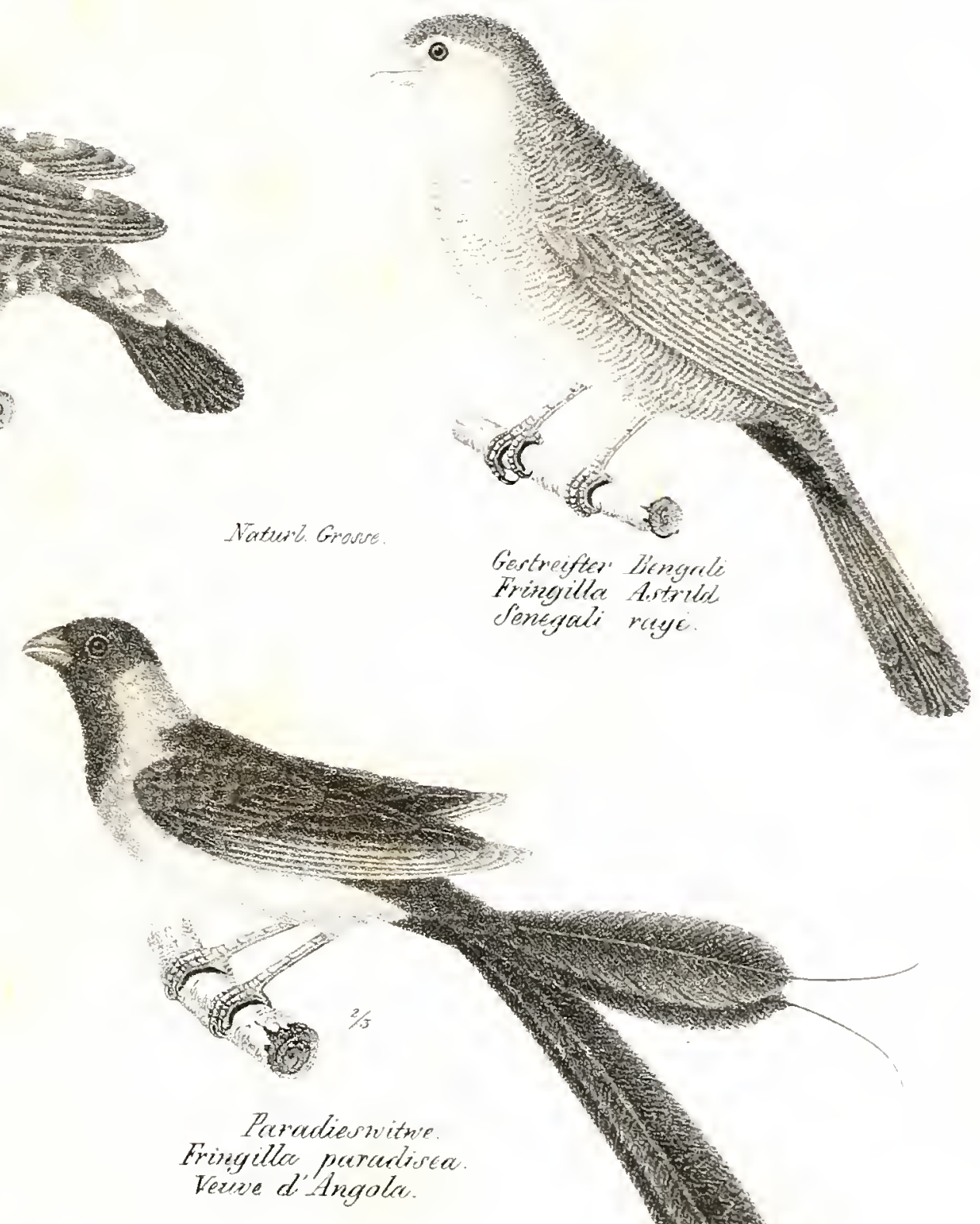

Dex Gemeine Gimpel.

Fringillse pyrrtiule.
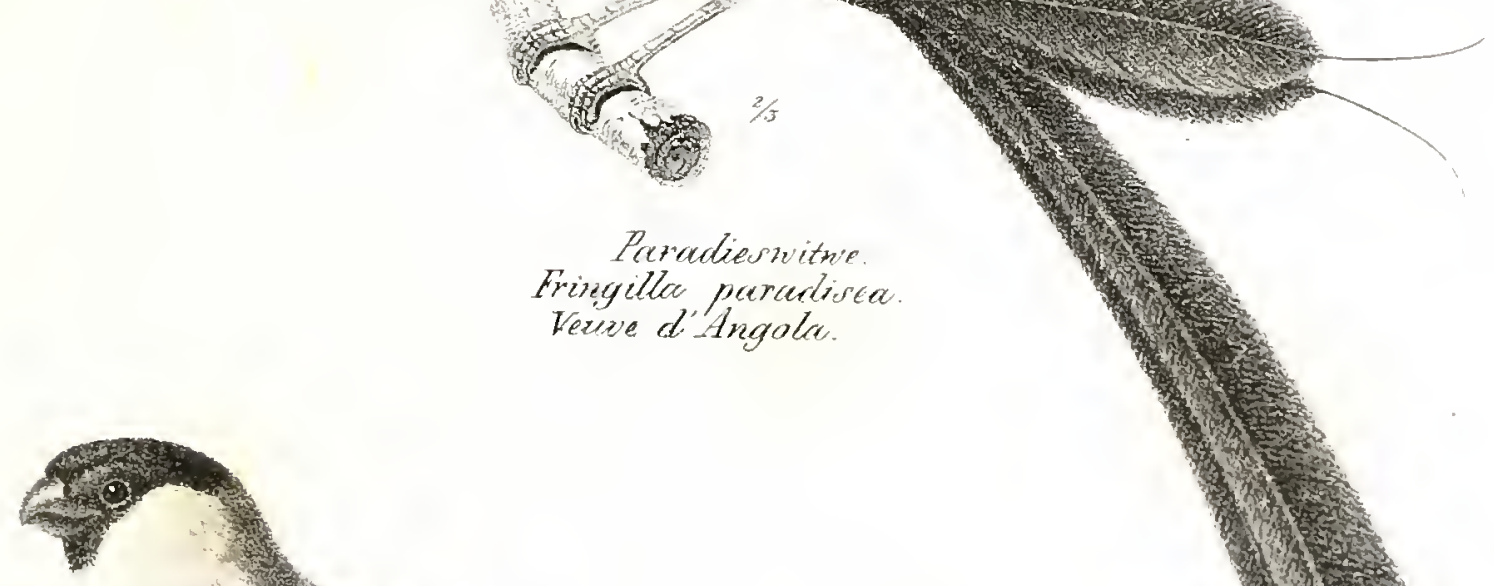

Le Dousermul commes
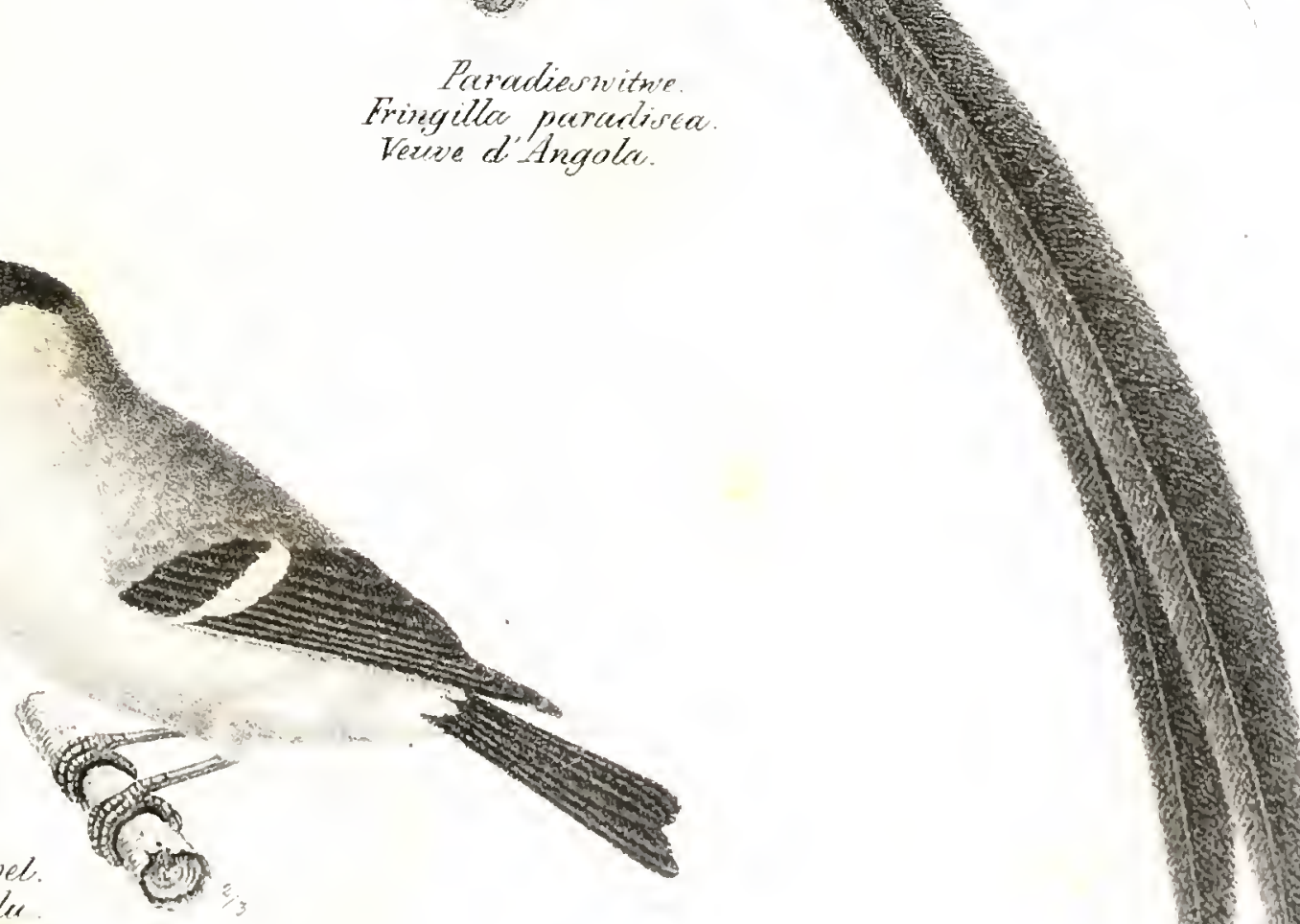


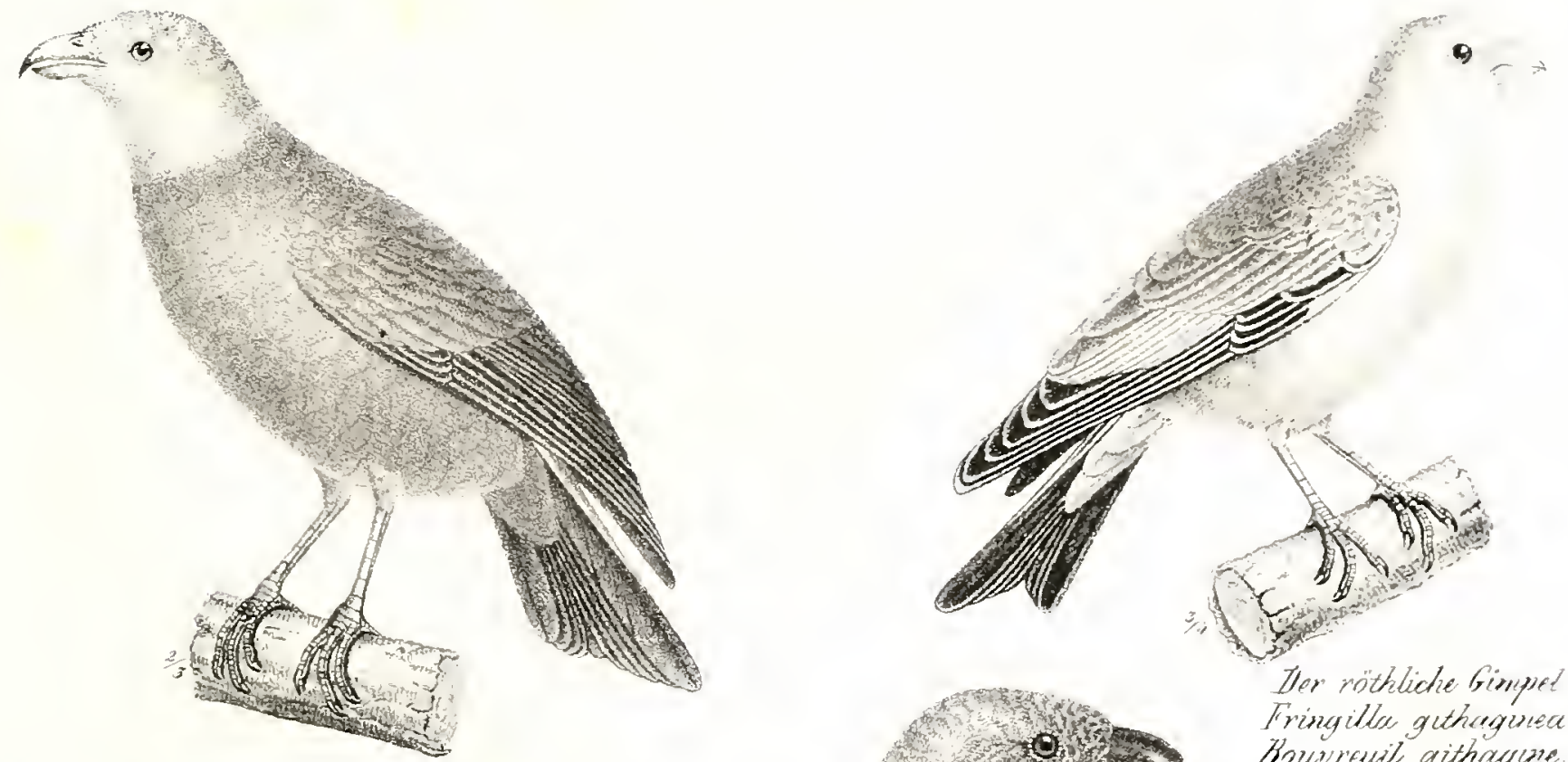

Gelbkiörufiger Paproysay-Schrabed Puittirastra ictercoseptrala Puttivastre icterncephale.

$+0$ 2. M

Fringille githrower. Bowvrevil, githagine
Gemeiner Firelsichorbel

Laren curviroitice Le bew crowe
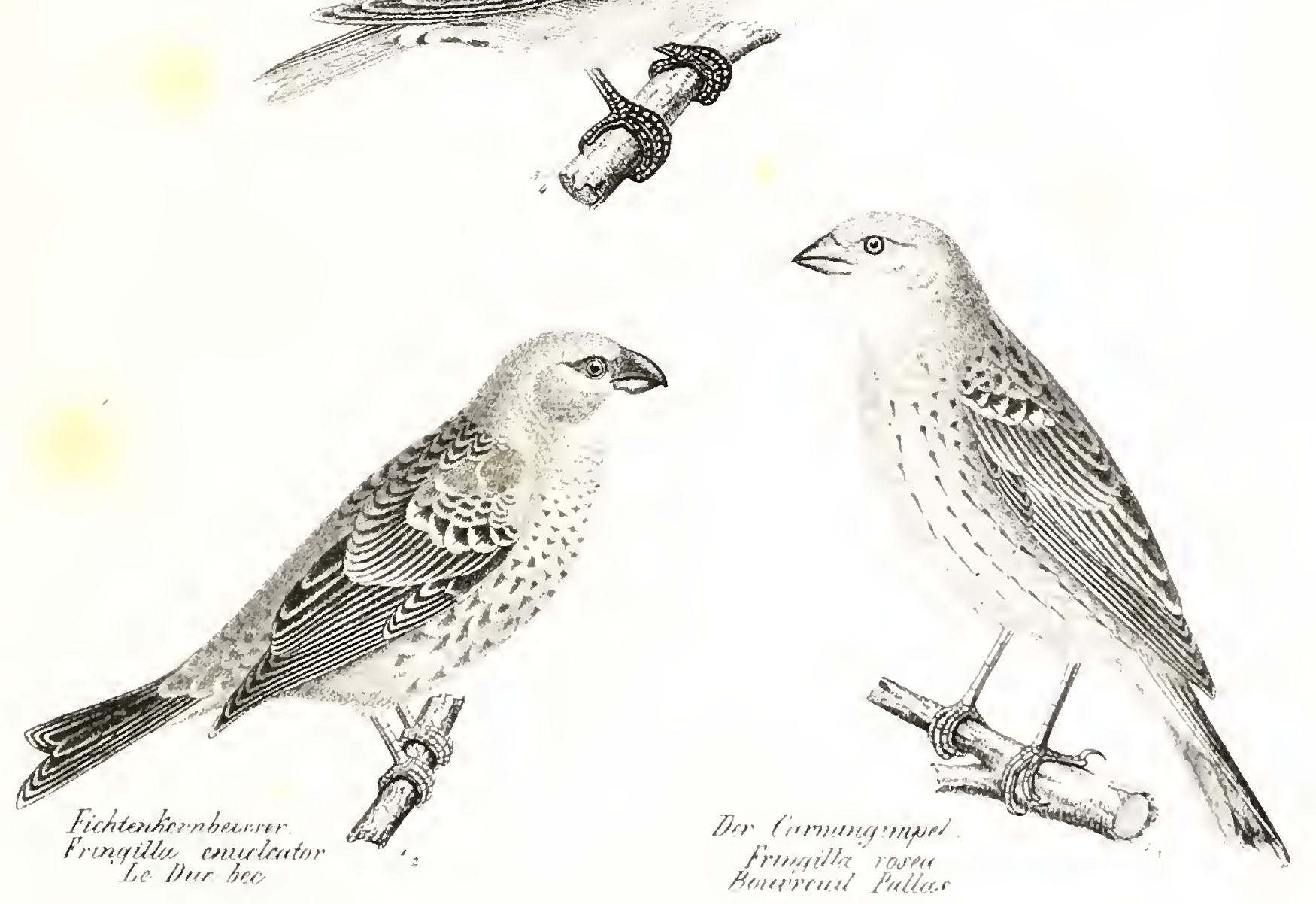



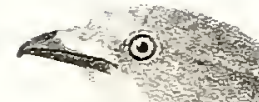

1.

(c)

$-1+2$

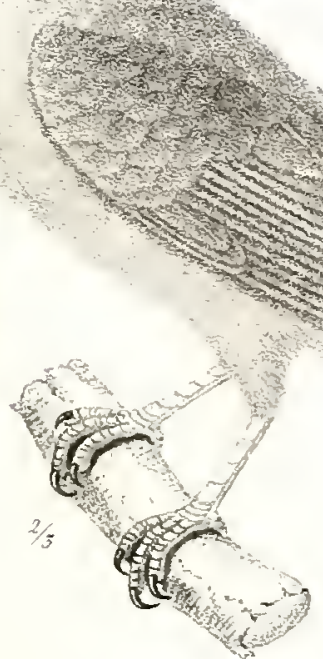

Dur indusche Regelschuatiel

Colius indictss.

Le colisis
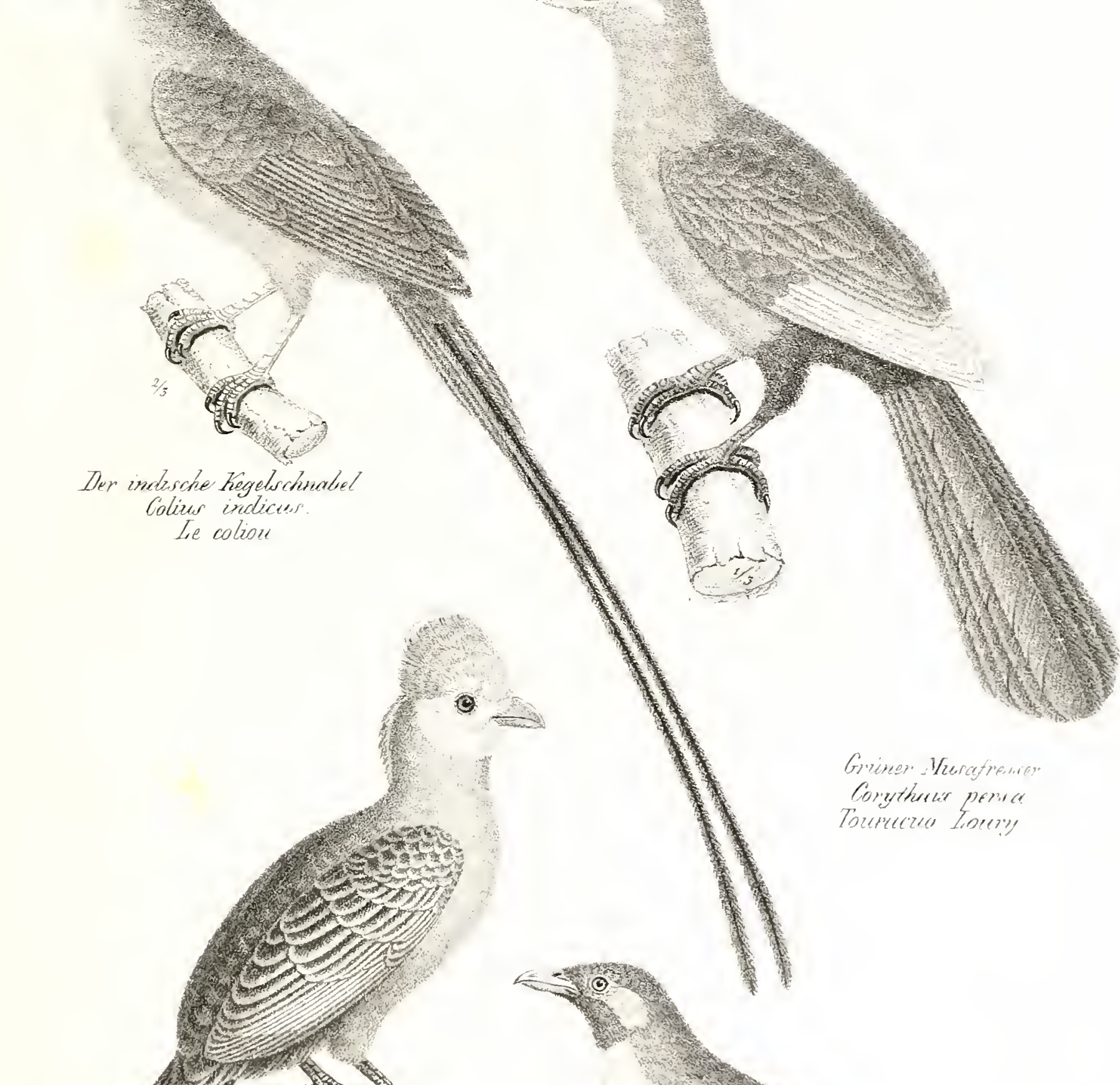


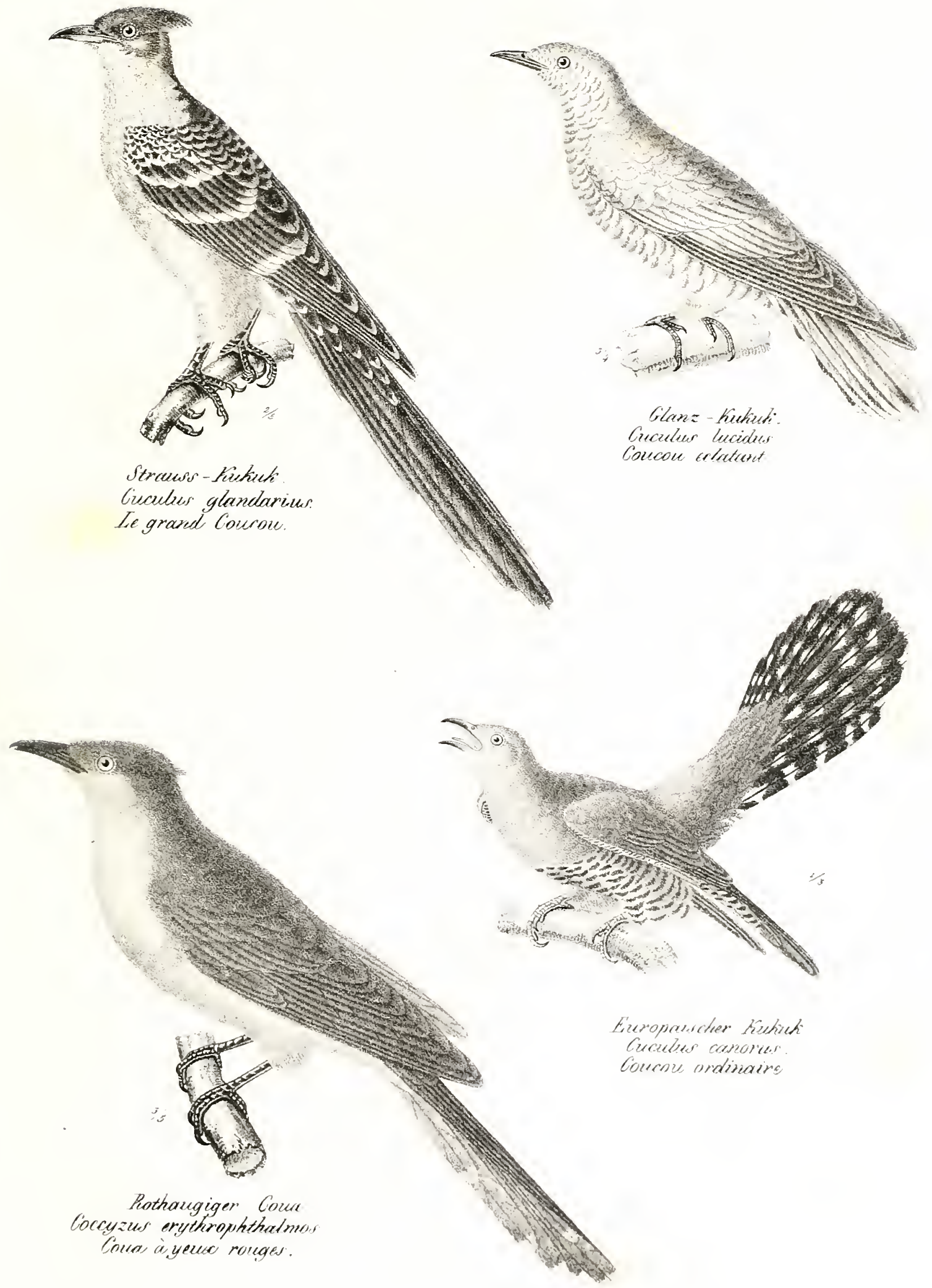

Fascase-Sporn-Bühuh

Centropues phexsianues.

Concal Faisan.
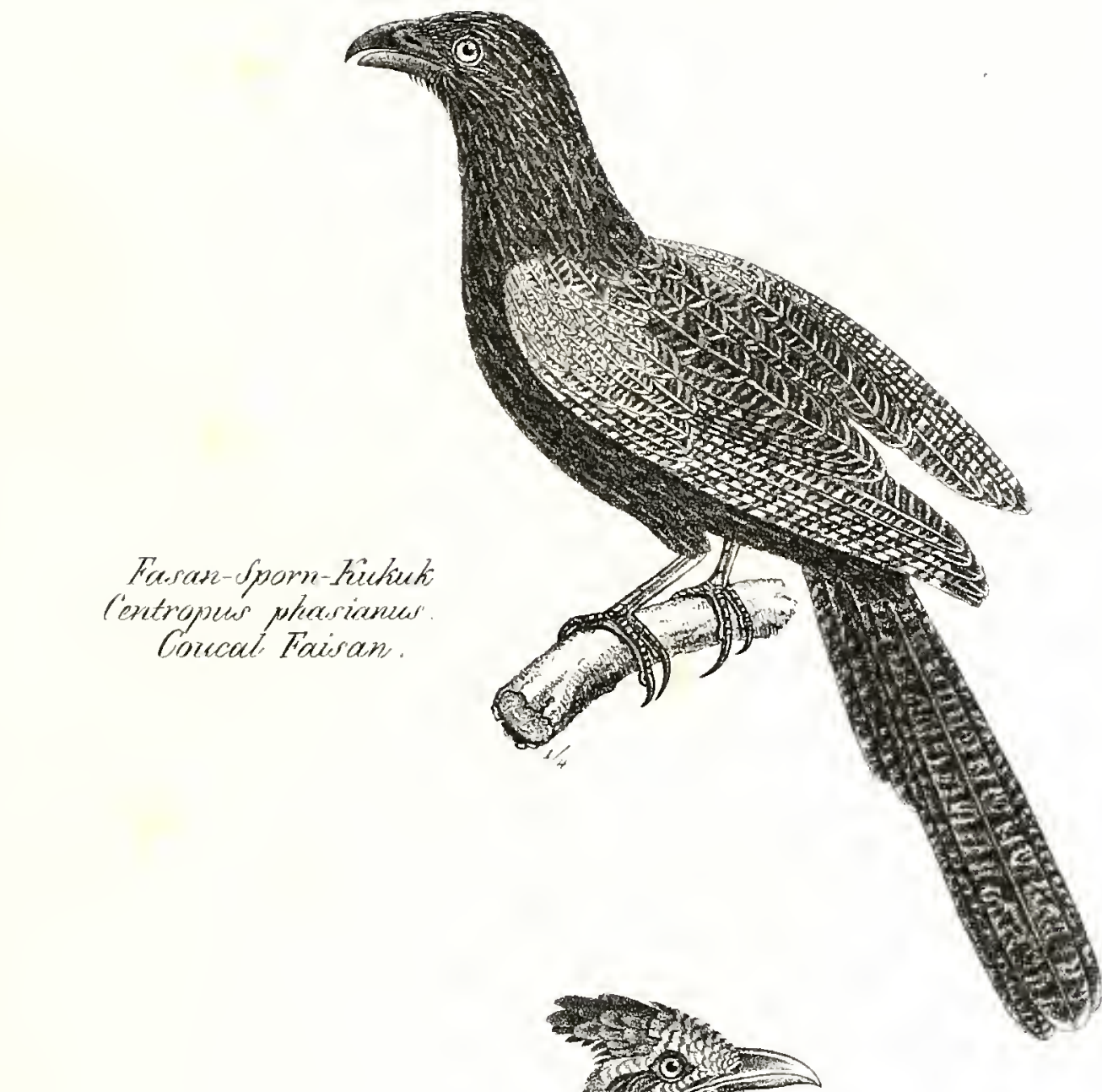

in

Hete?
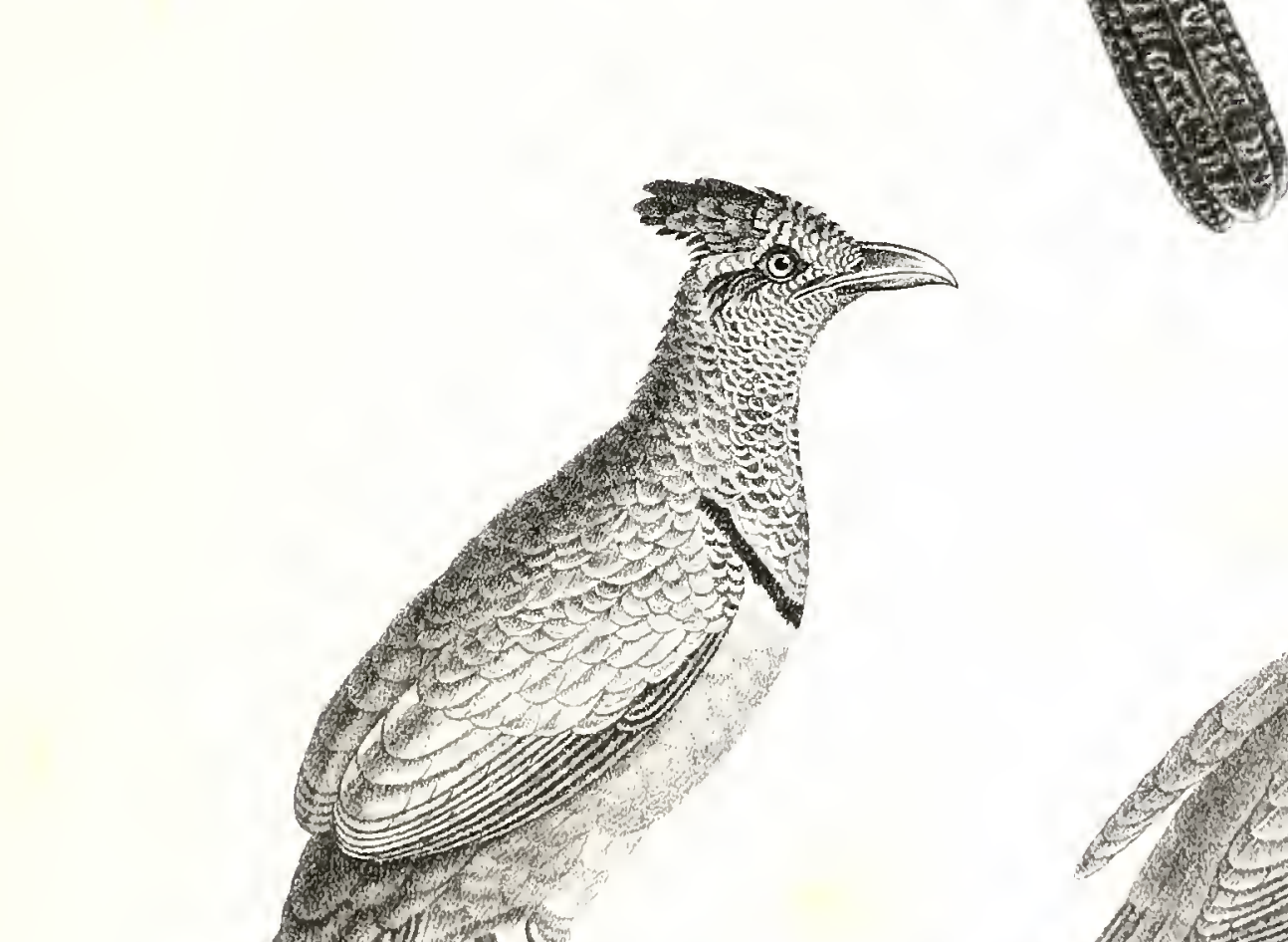


$$
\text { . }
$$


Neuthallandischer Rinnemschwatsed. sasthrops novae Hollandiau Soythrops presager.

(c)
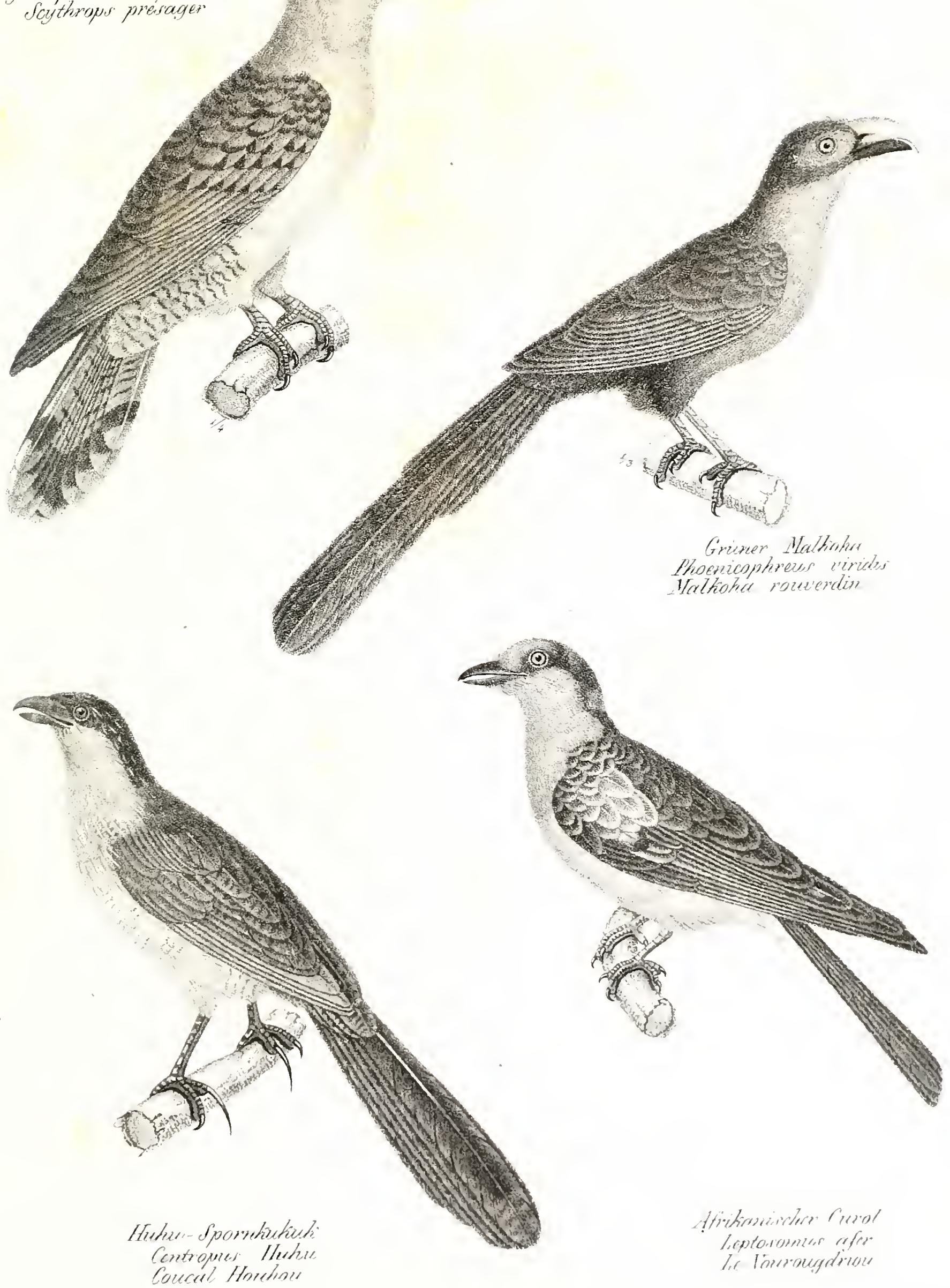


$$
\text { , }
$$




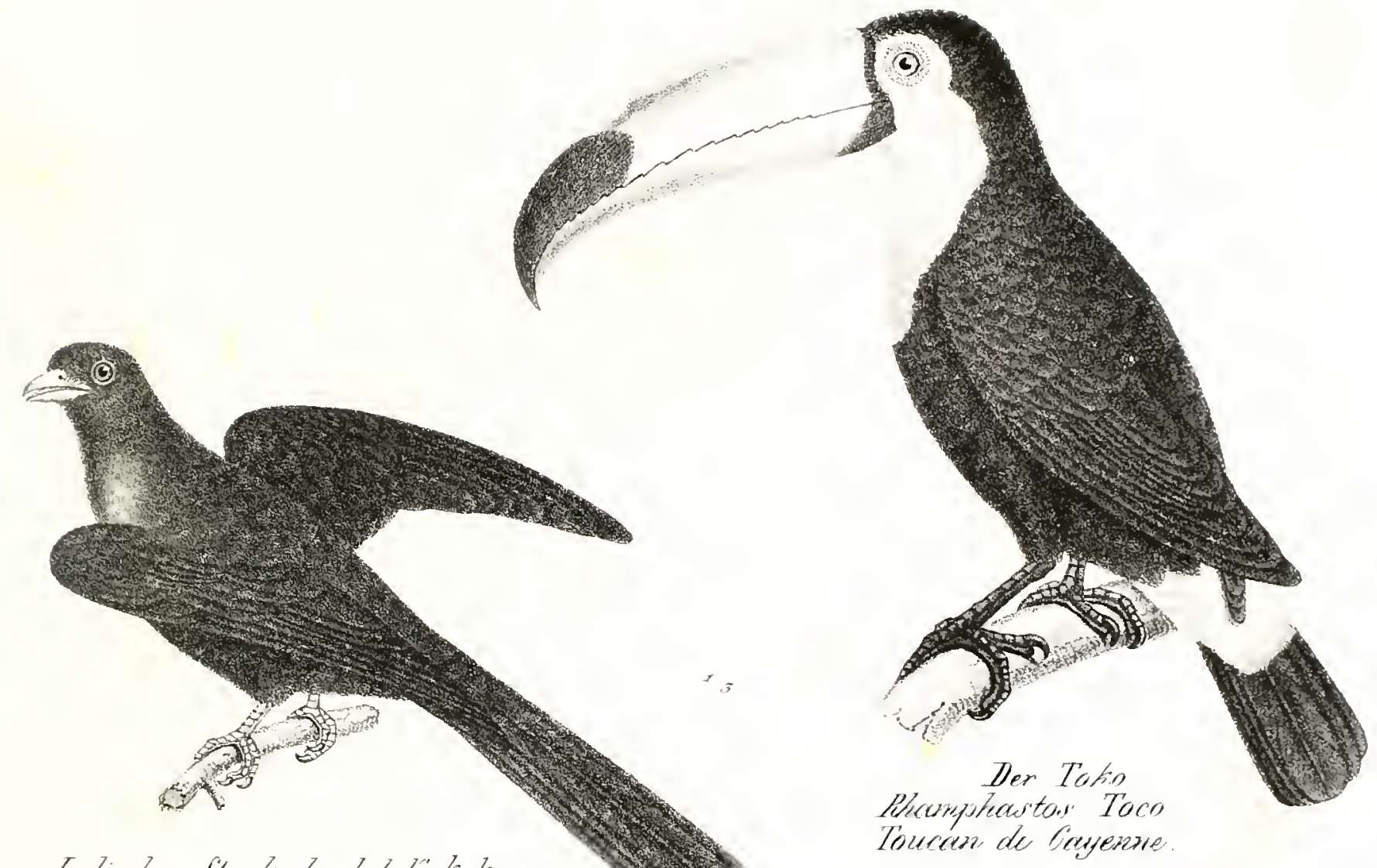

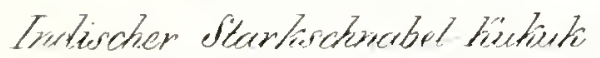

Endapentis orientritio.

Coucou des Inderi.

(2)

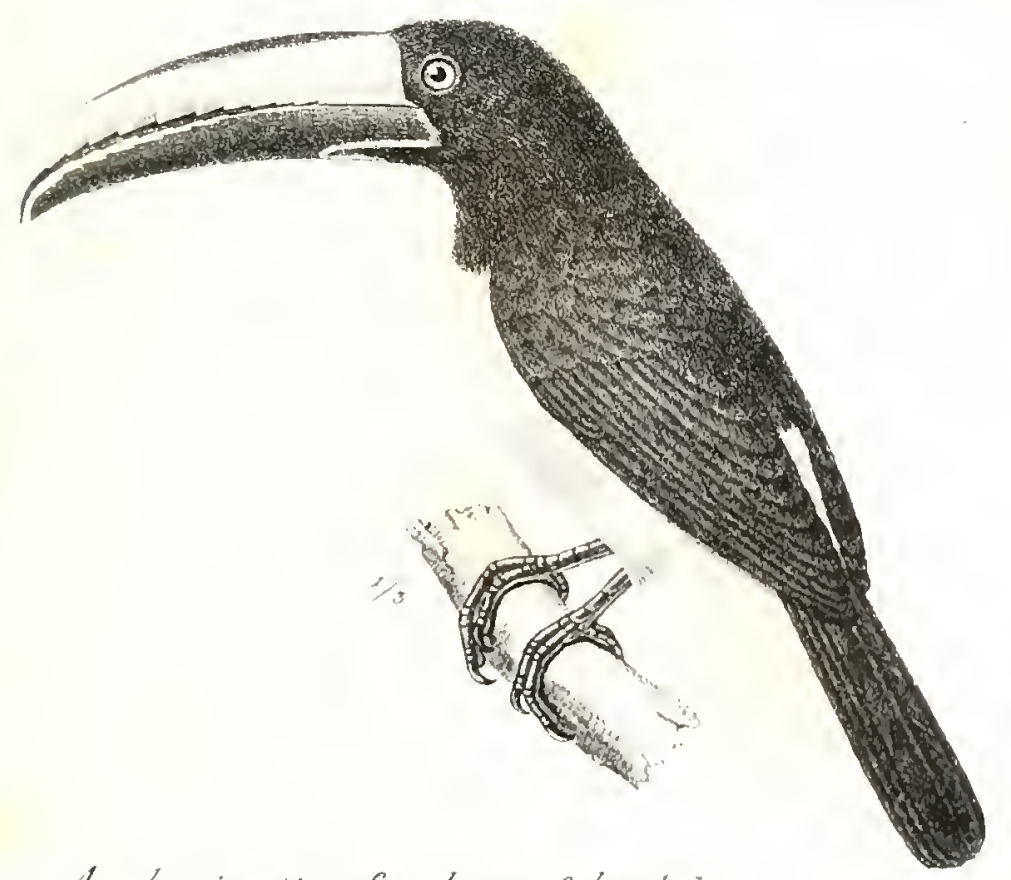

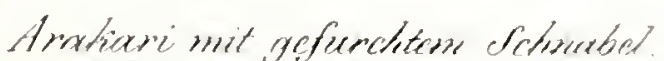

Ptoraglavione. creloxatus

Arecari iclorné

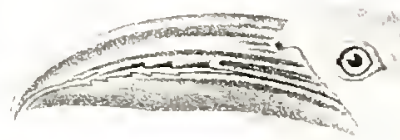

Phermpleaston' Taco

Toucuse de Geusense. 


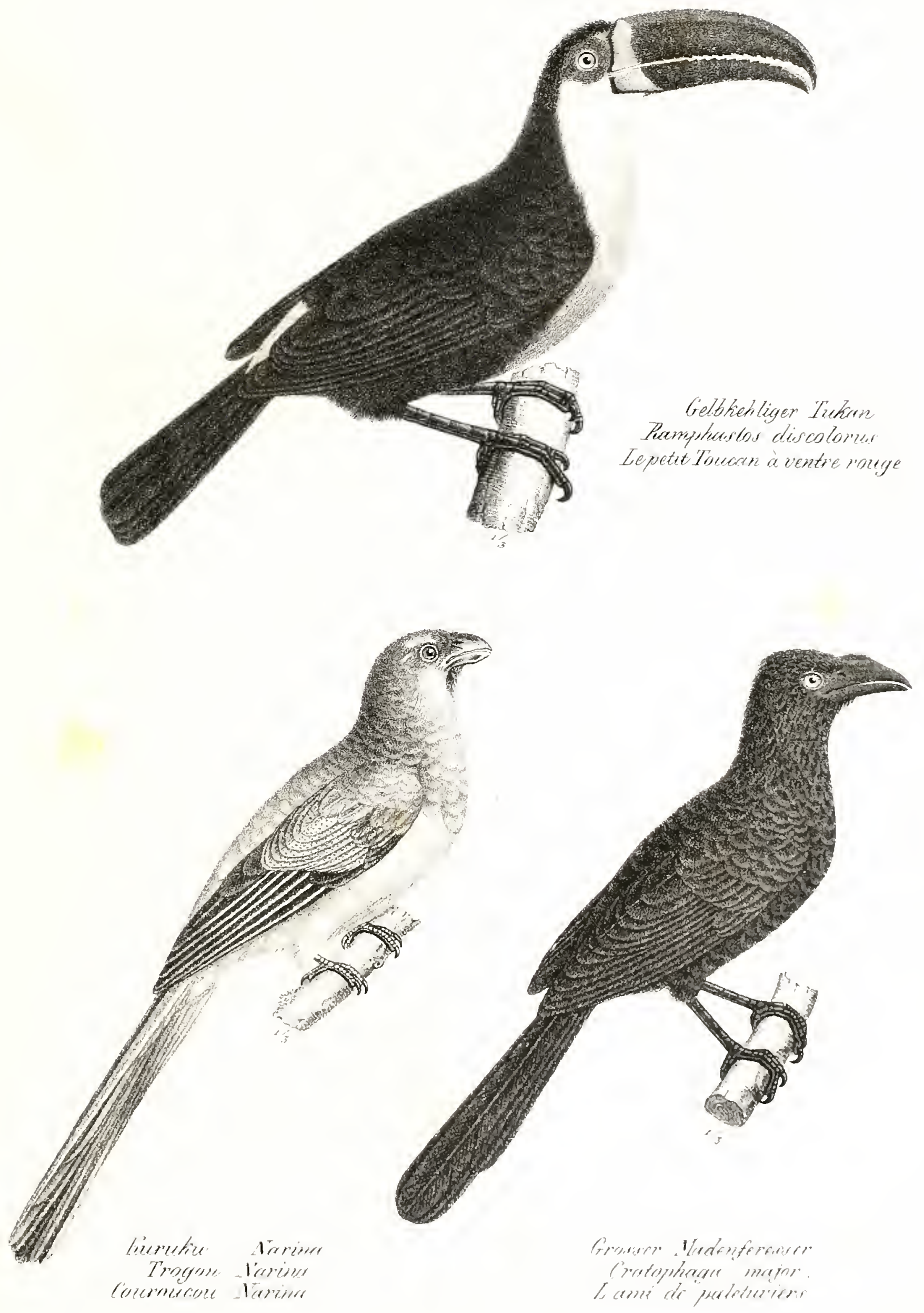


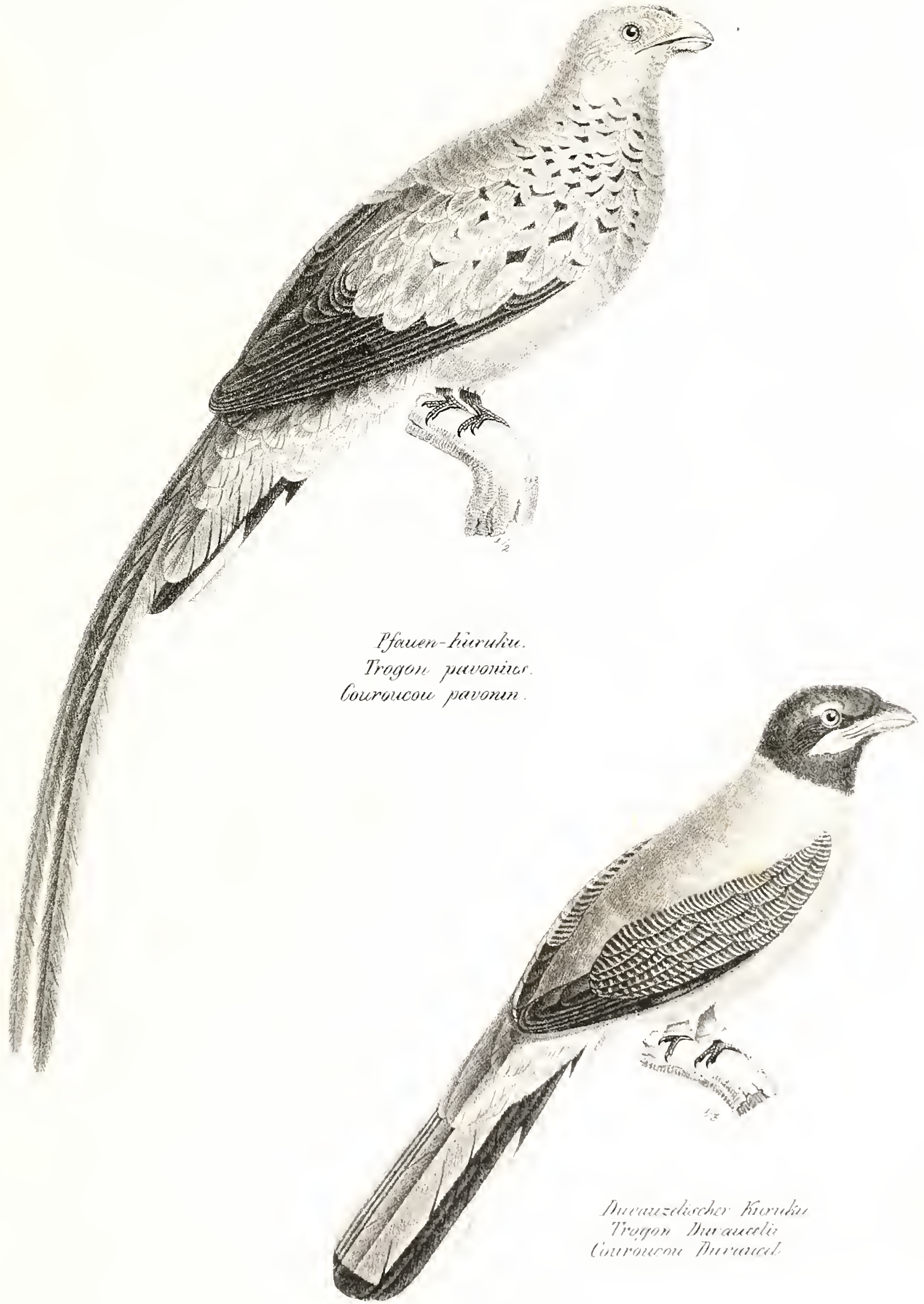




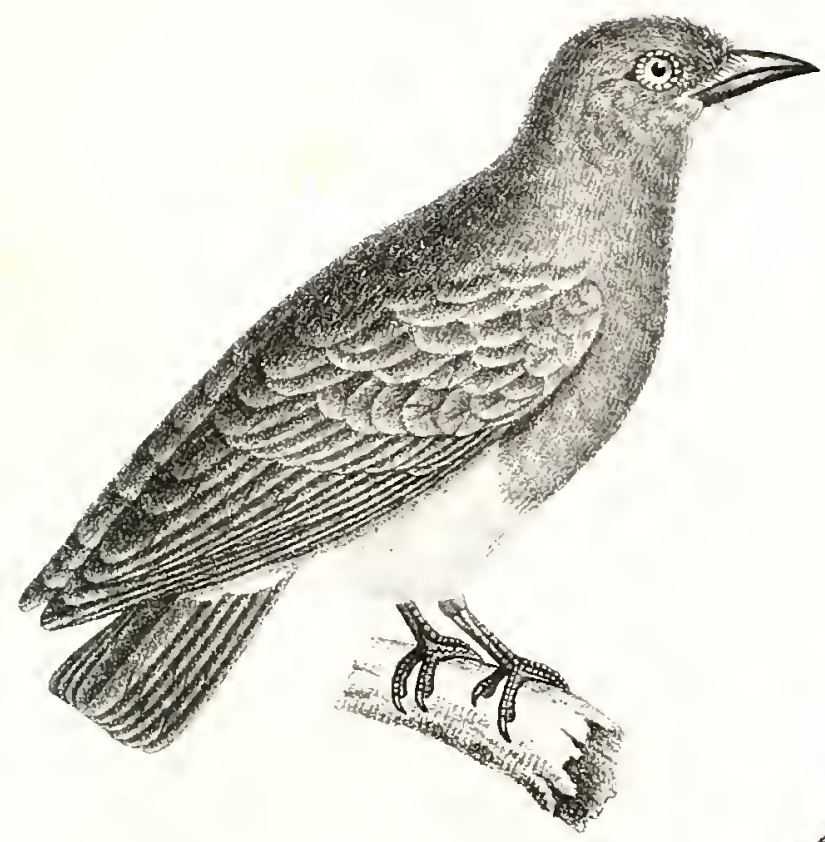

Duritiles. Bart-huhuh Monasa tenebrosa Barburm temébreu.
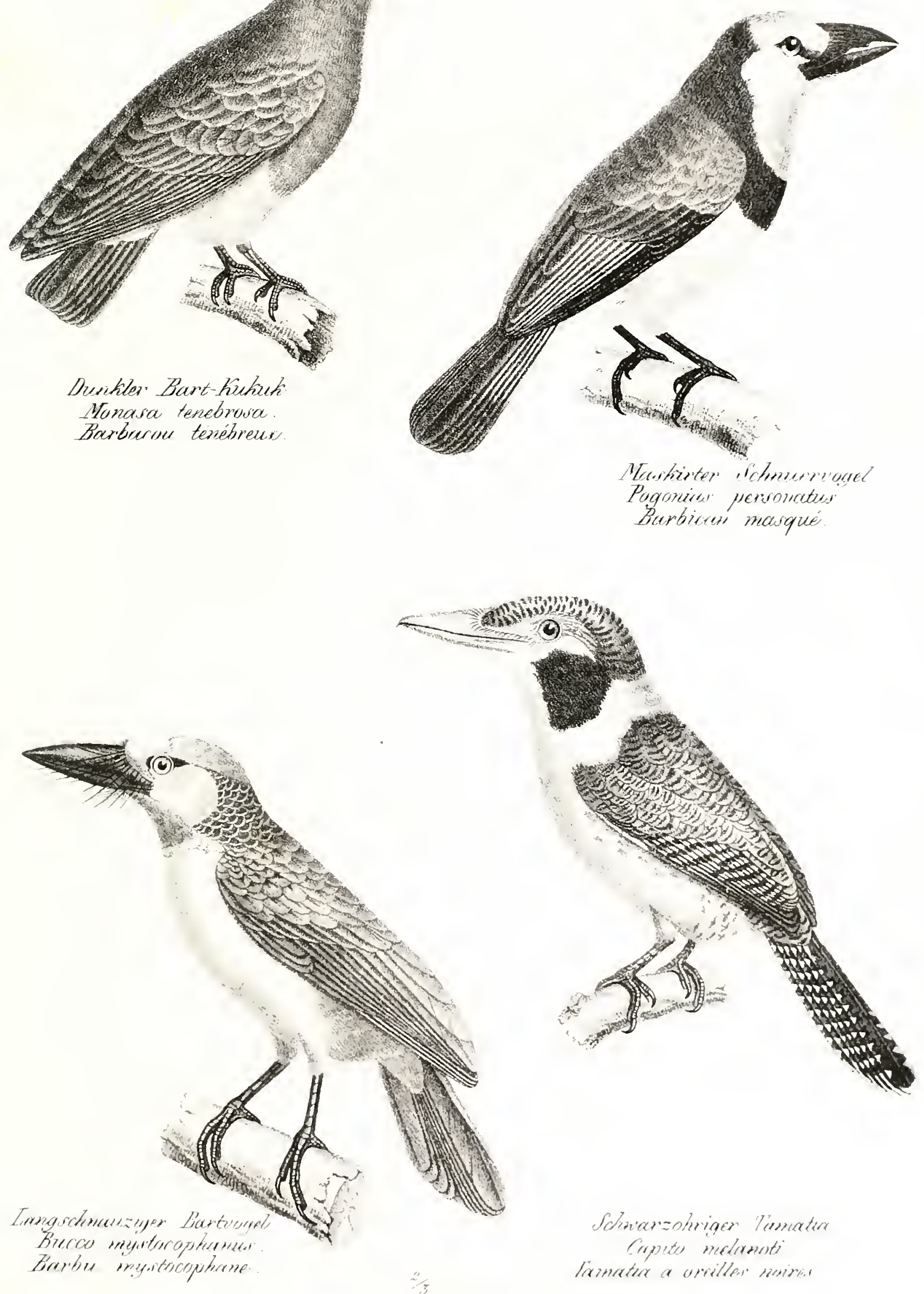


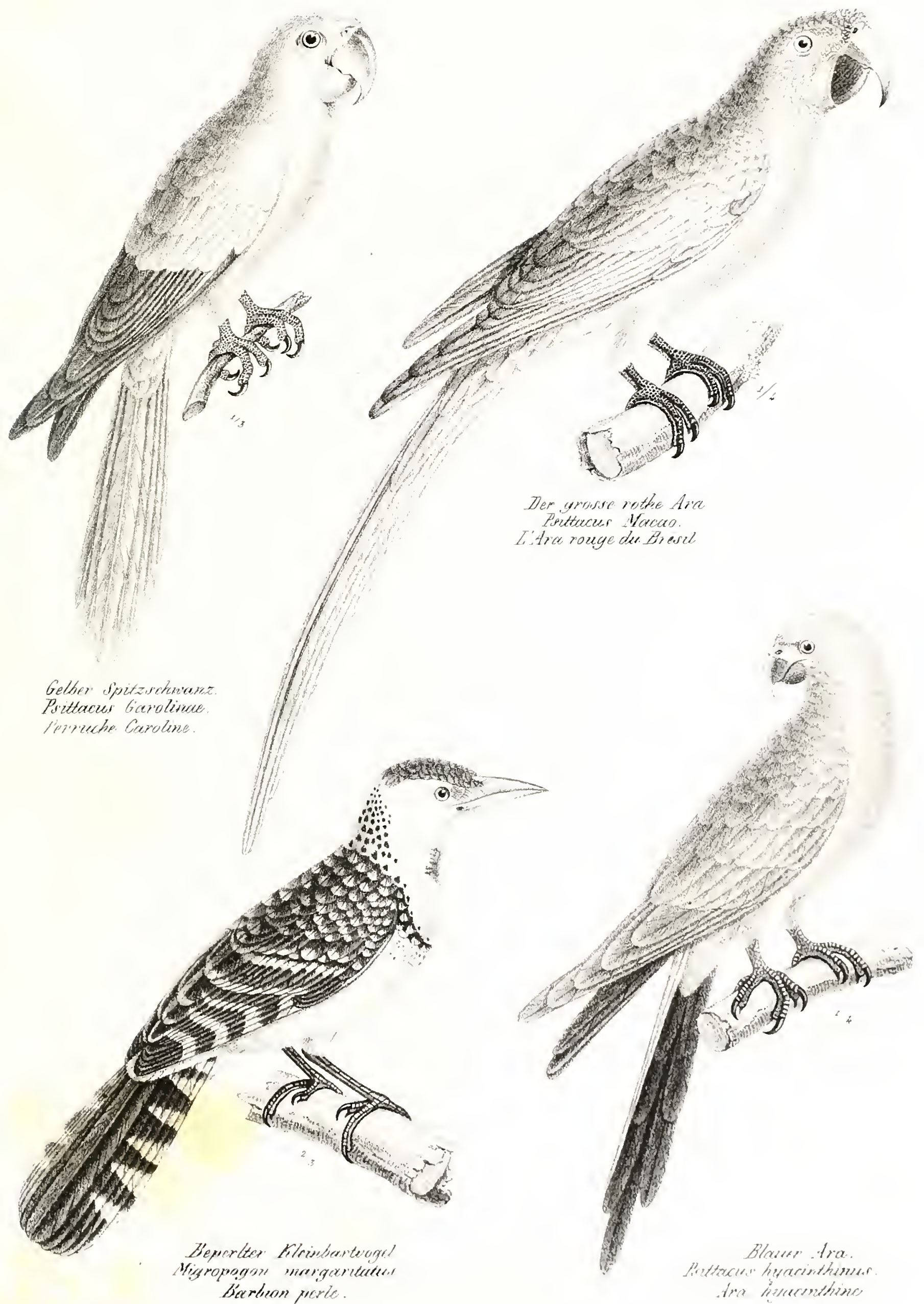




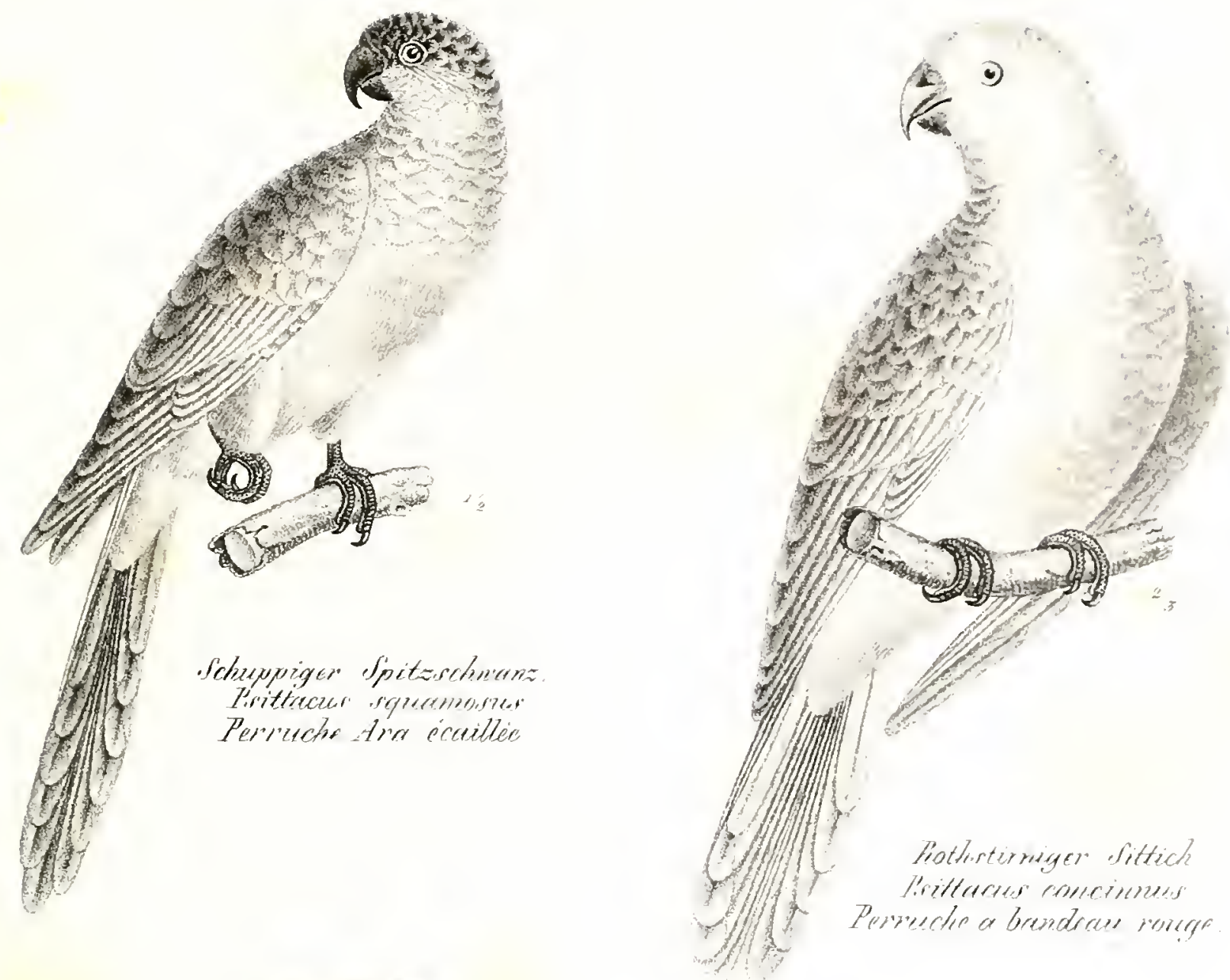

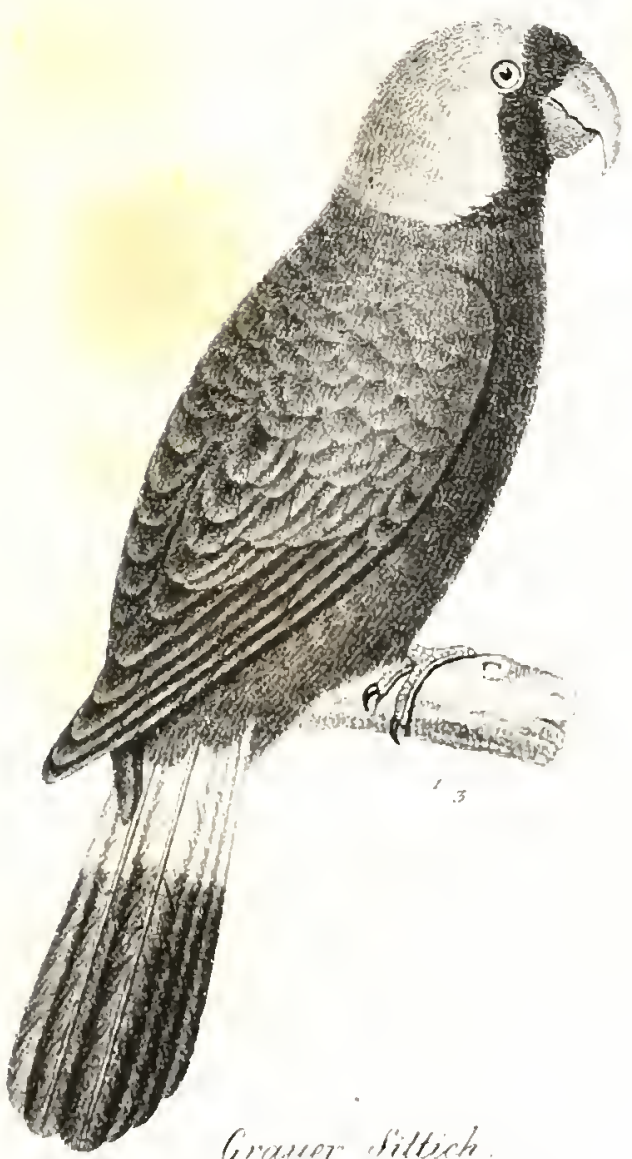

Pirelleseses surercasidmes Perrogenet meserarise

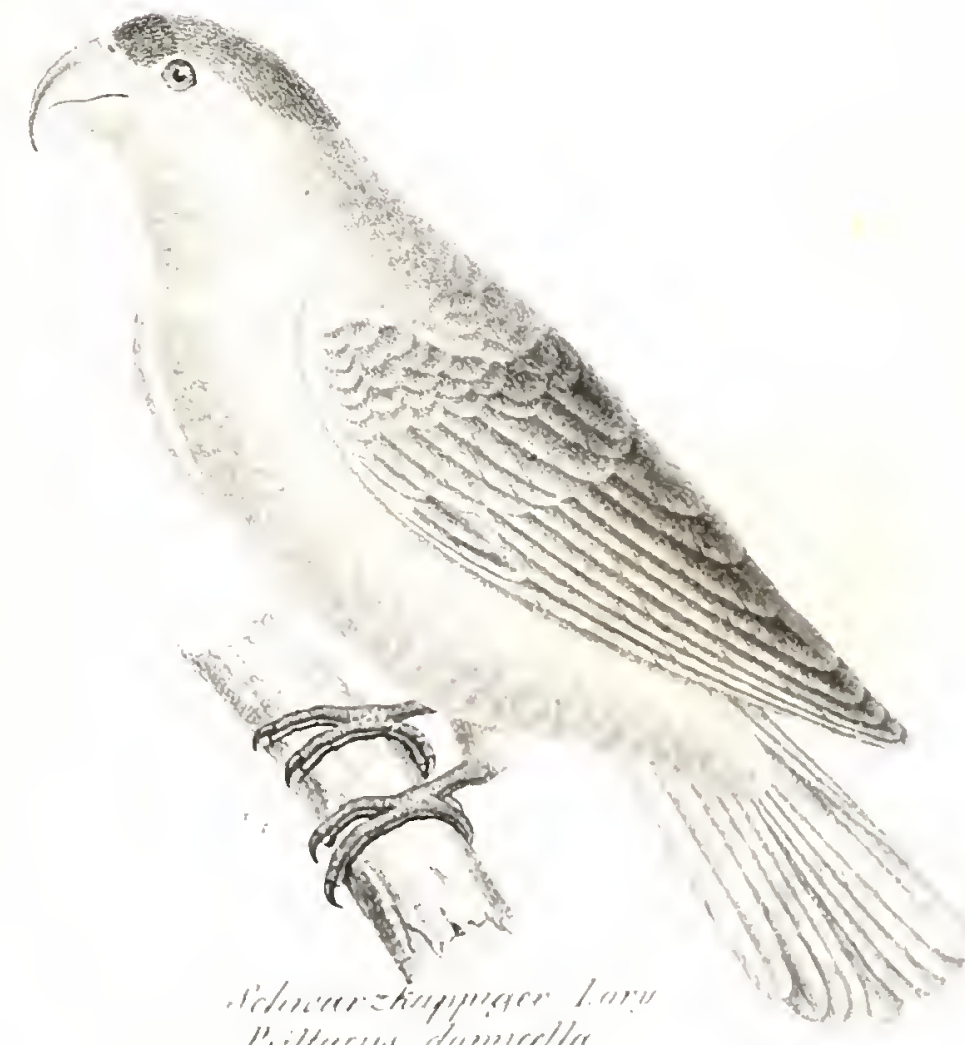

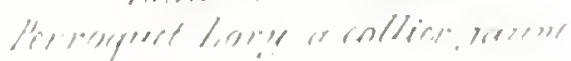


. 


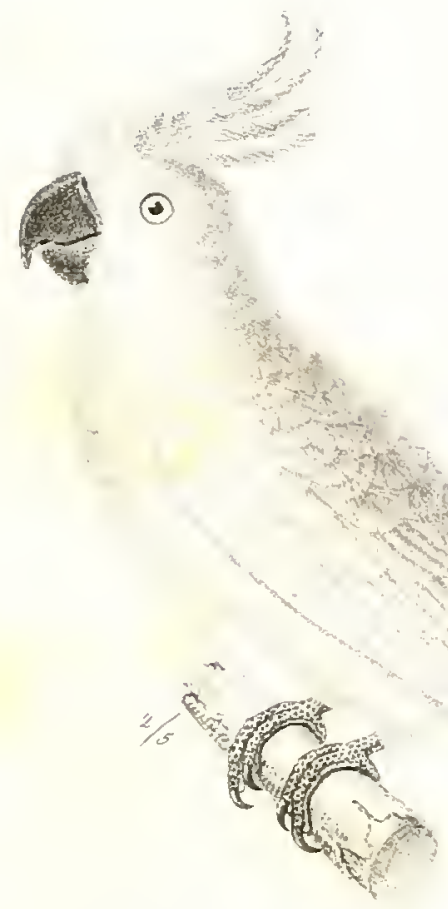

baskudu mits stwwefelyelbern Fedenbus ch. Psittrecus sulpoteurenes. fiatialoes à hoppse joutse

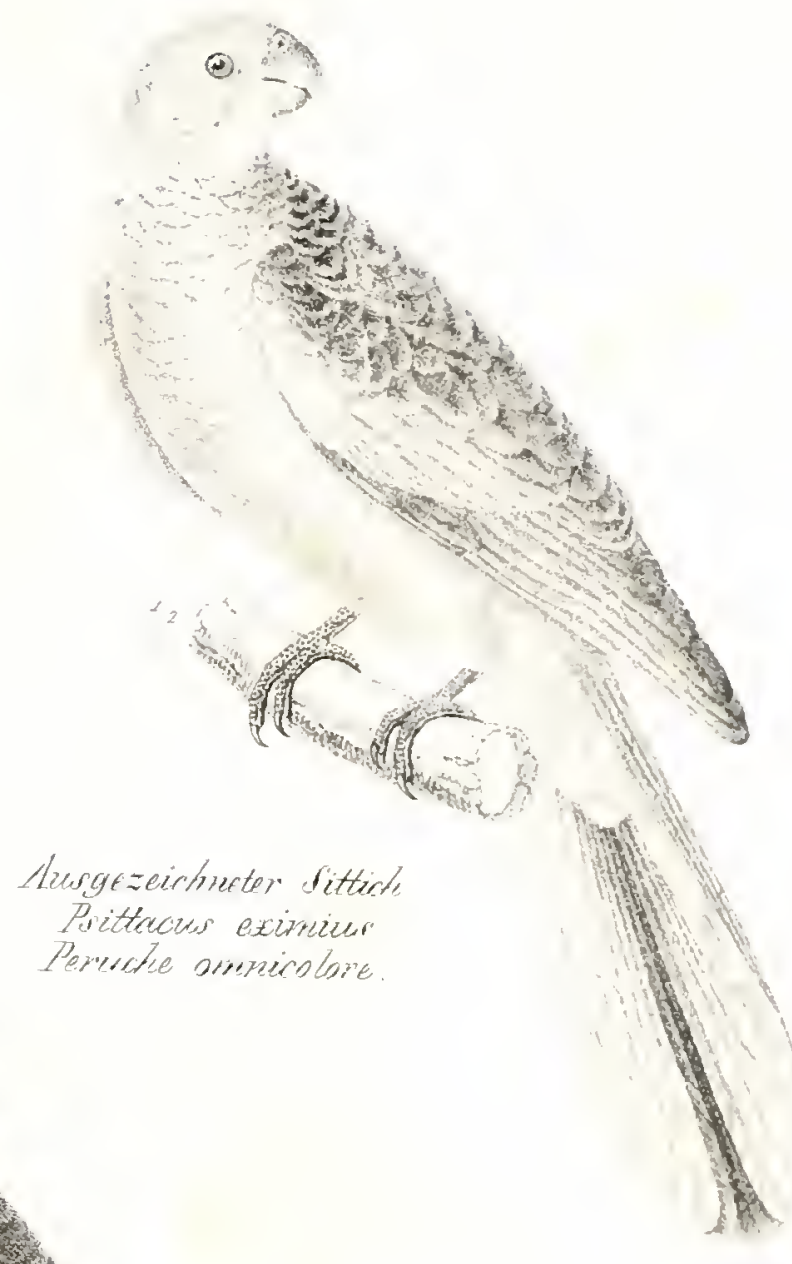

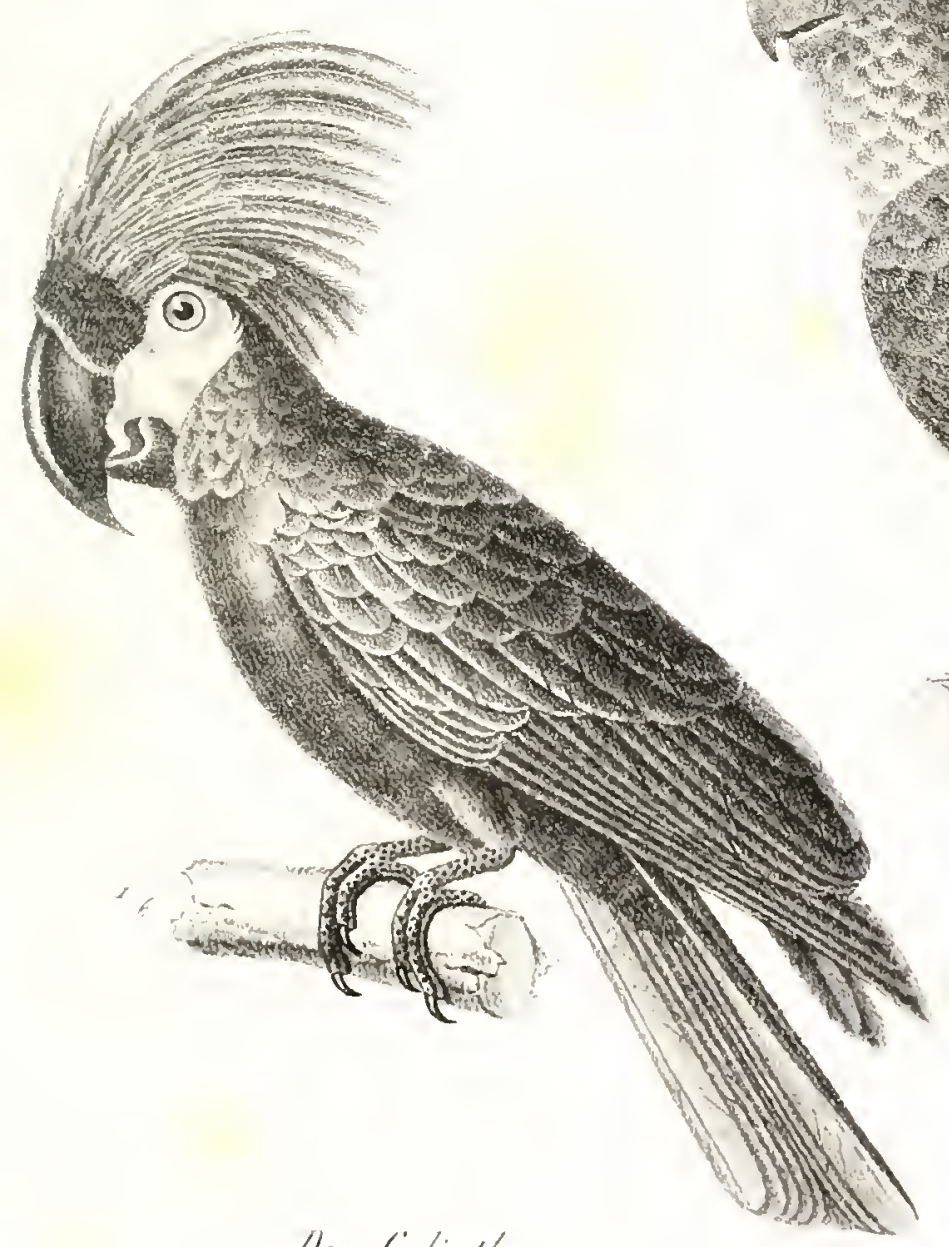

Der Gioliced,

Pinttarine Golirsth

- traegres a Irompe

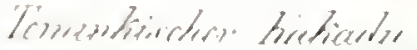

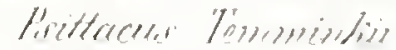

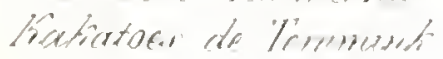




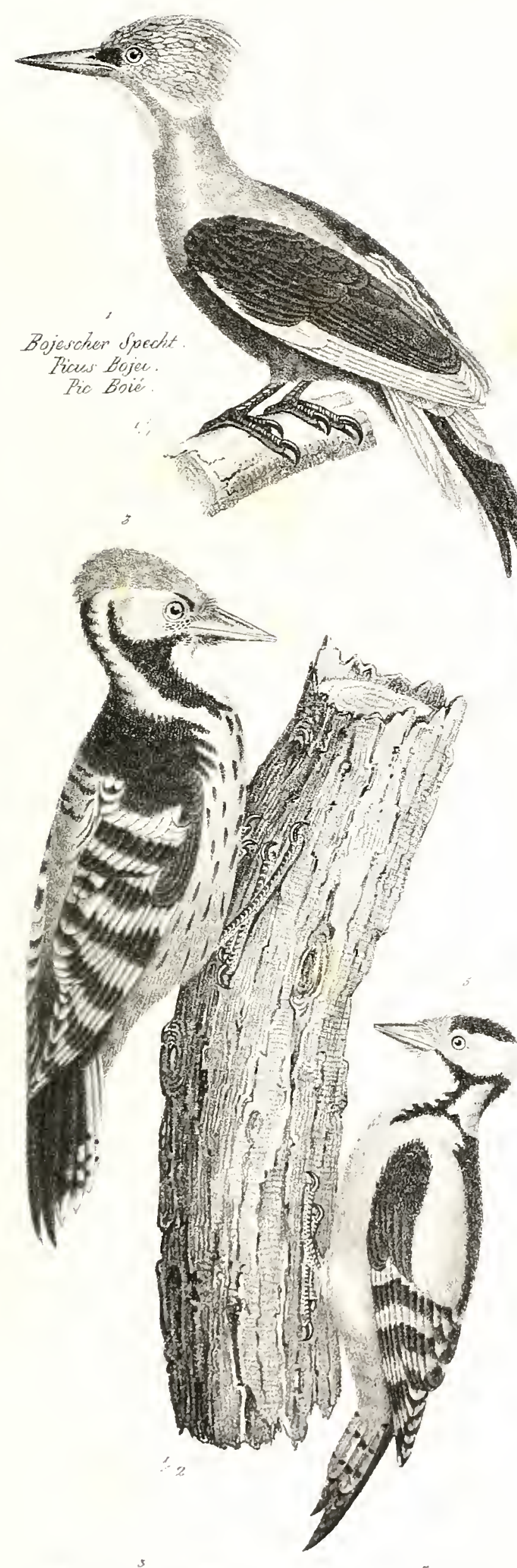

Wrosisruetigger Apucedit

Pecies lenesomeres.

Pic lecesonets
Cimeanspecte

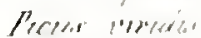

Le. lis neet
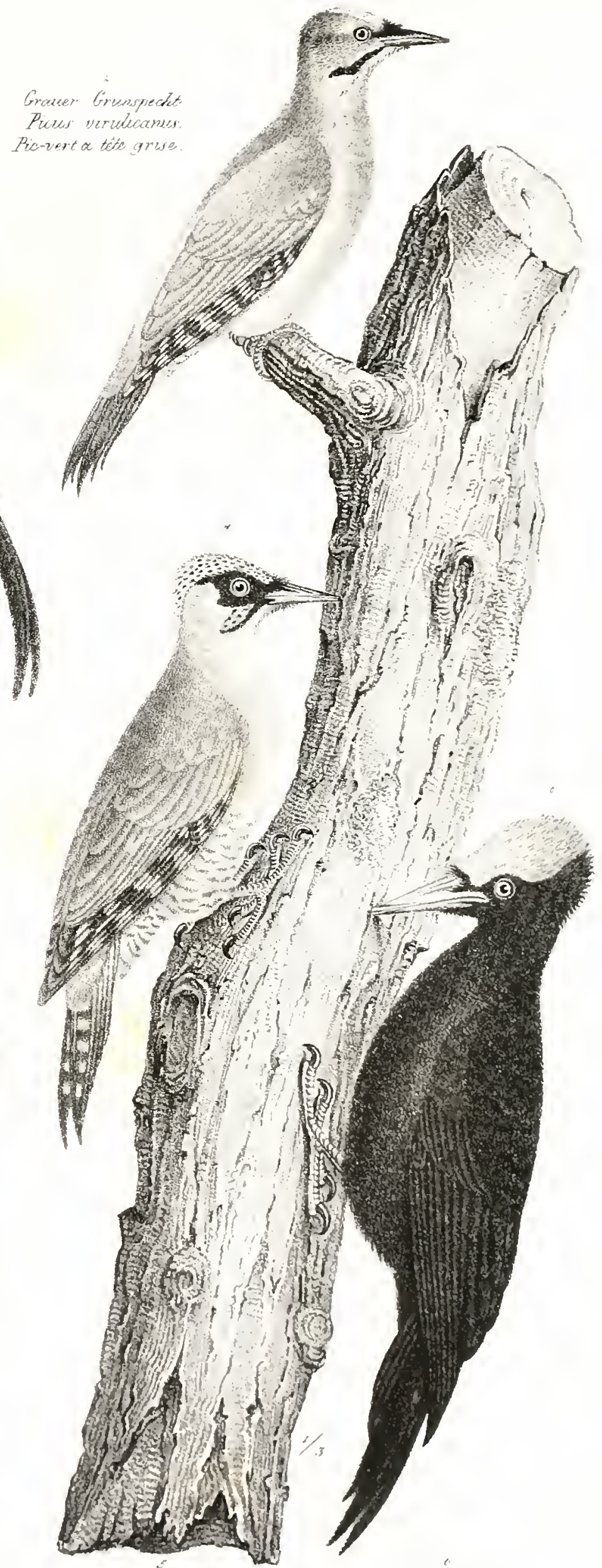

Groviser Riconaspecont

Prexa naymo.

Pre-Cpererie

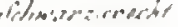

If P P W 
Langsetenvounziger Tathamar. Galbula paradisca. Soxcomar a longue quexes.
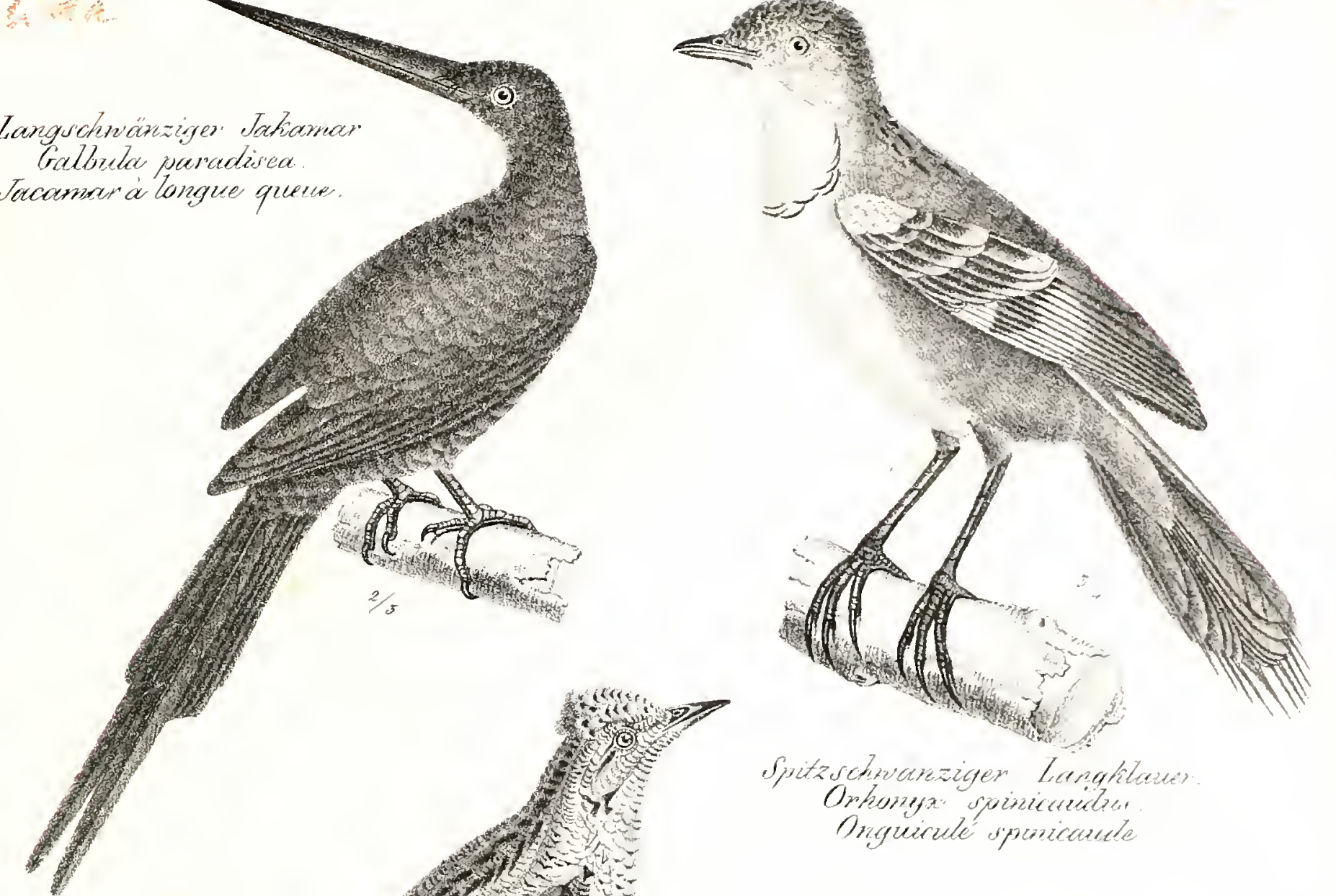

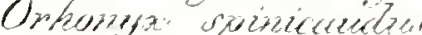

Onguirulé sponicusete

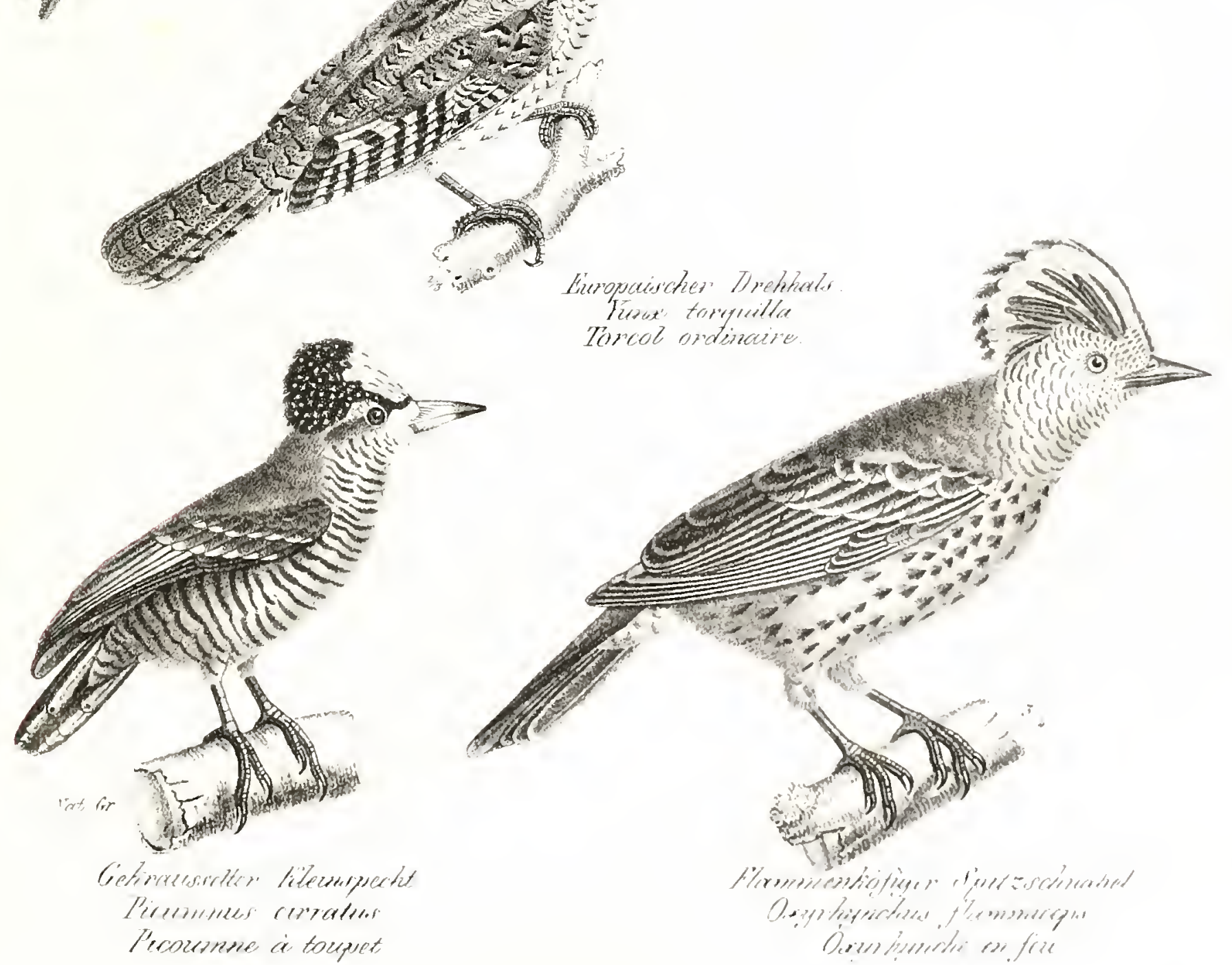




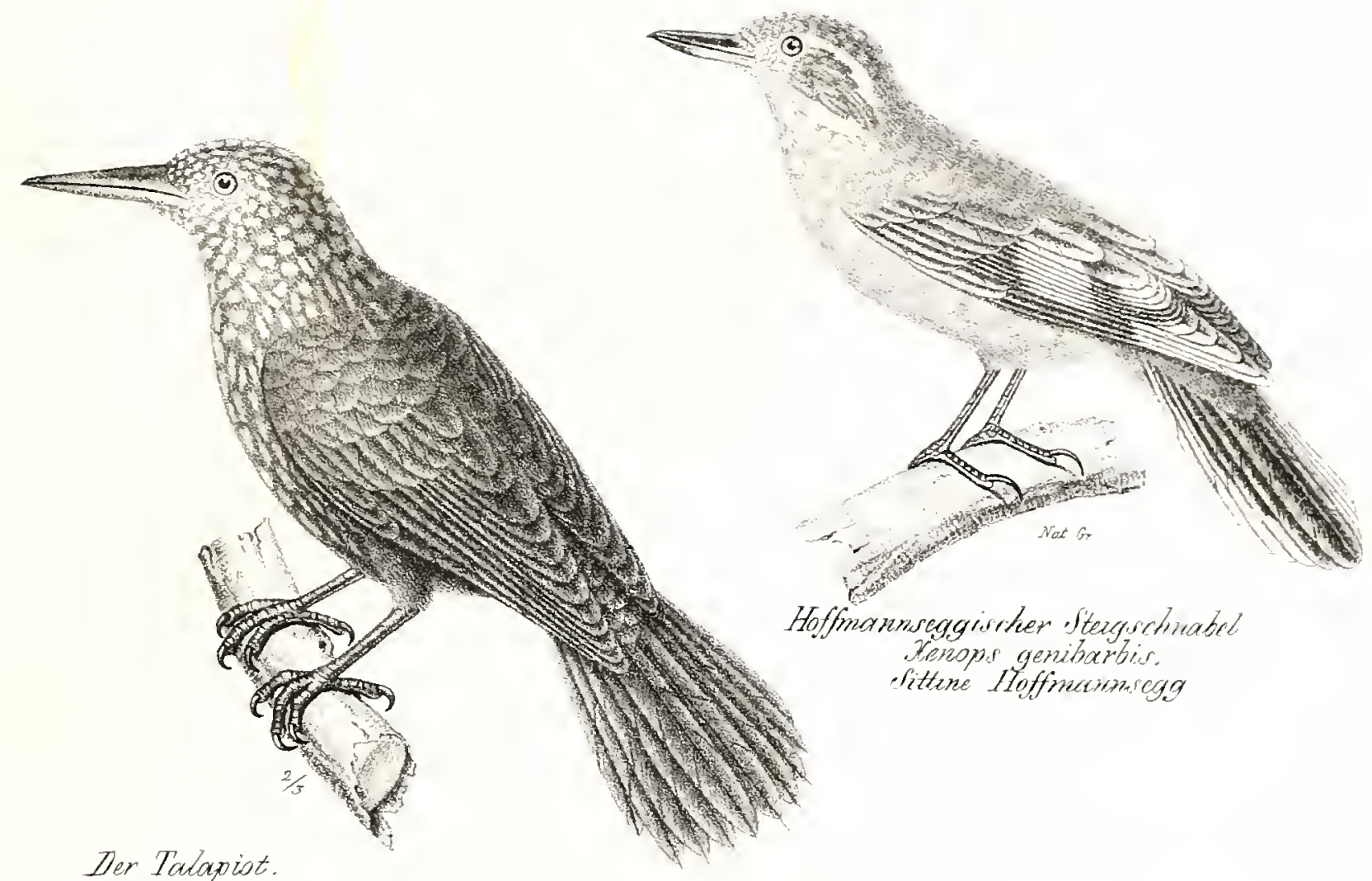

Denetrocaleptes picas.

Picusule Talapint

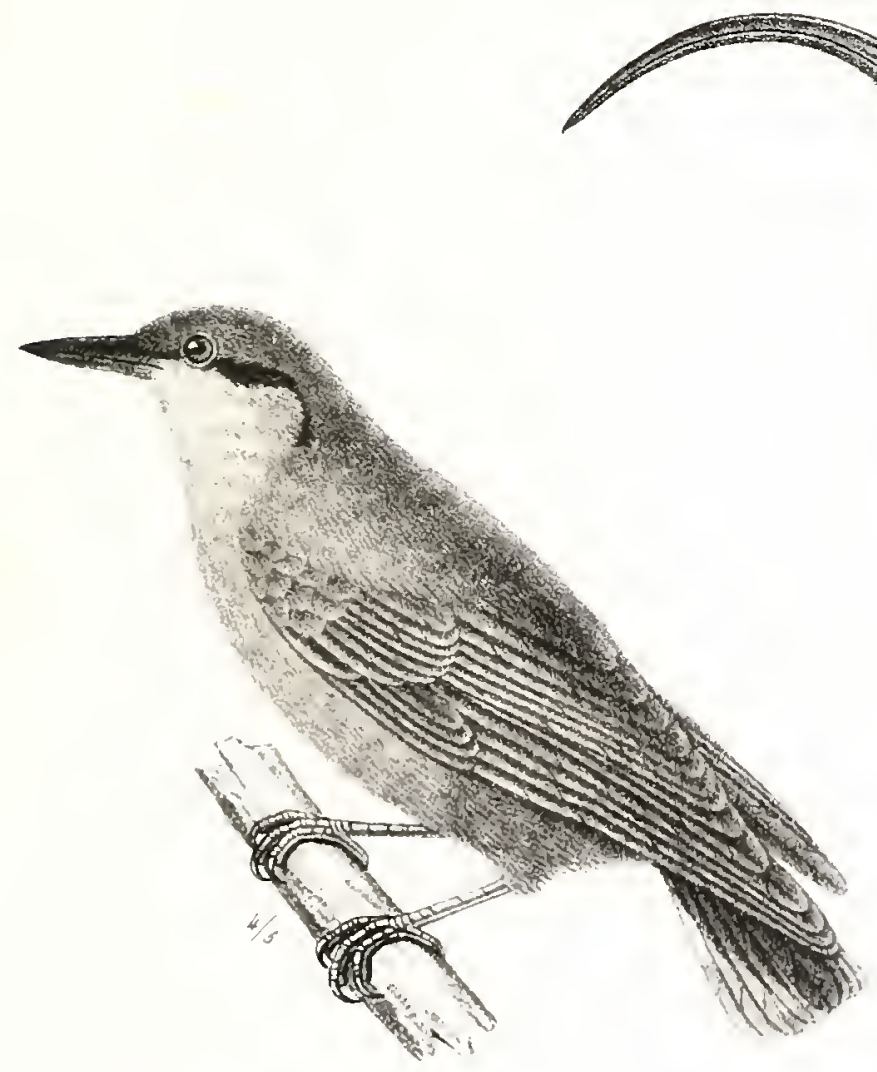

Eiverapaitische elpechtimstise

dille semopusere

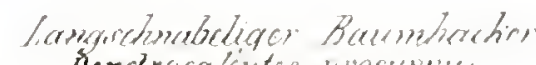

situlle toretecpoit.

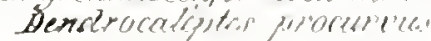

Grimpicer promsiopin

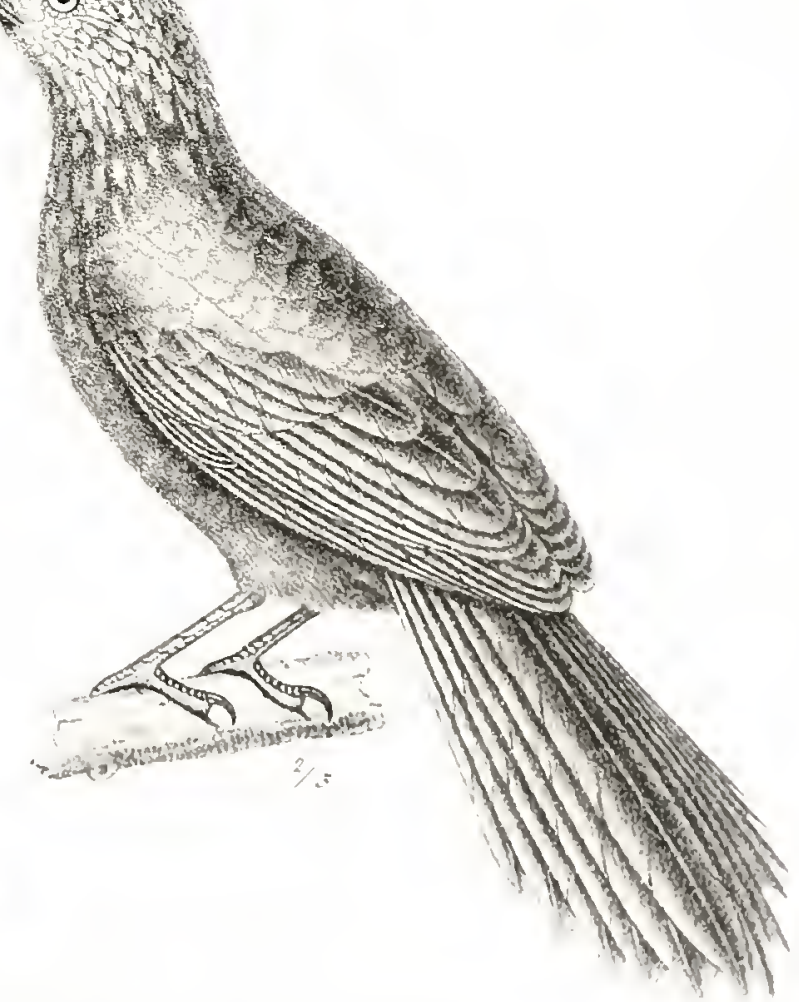




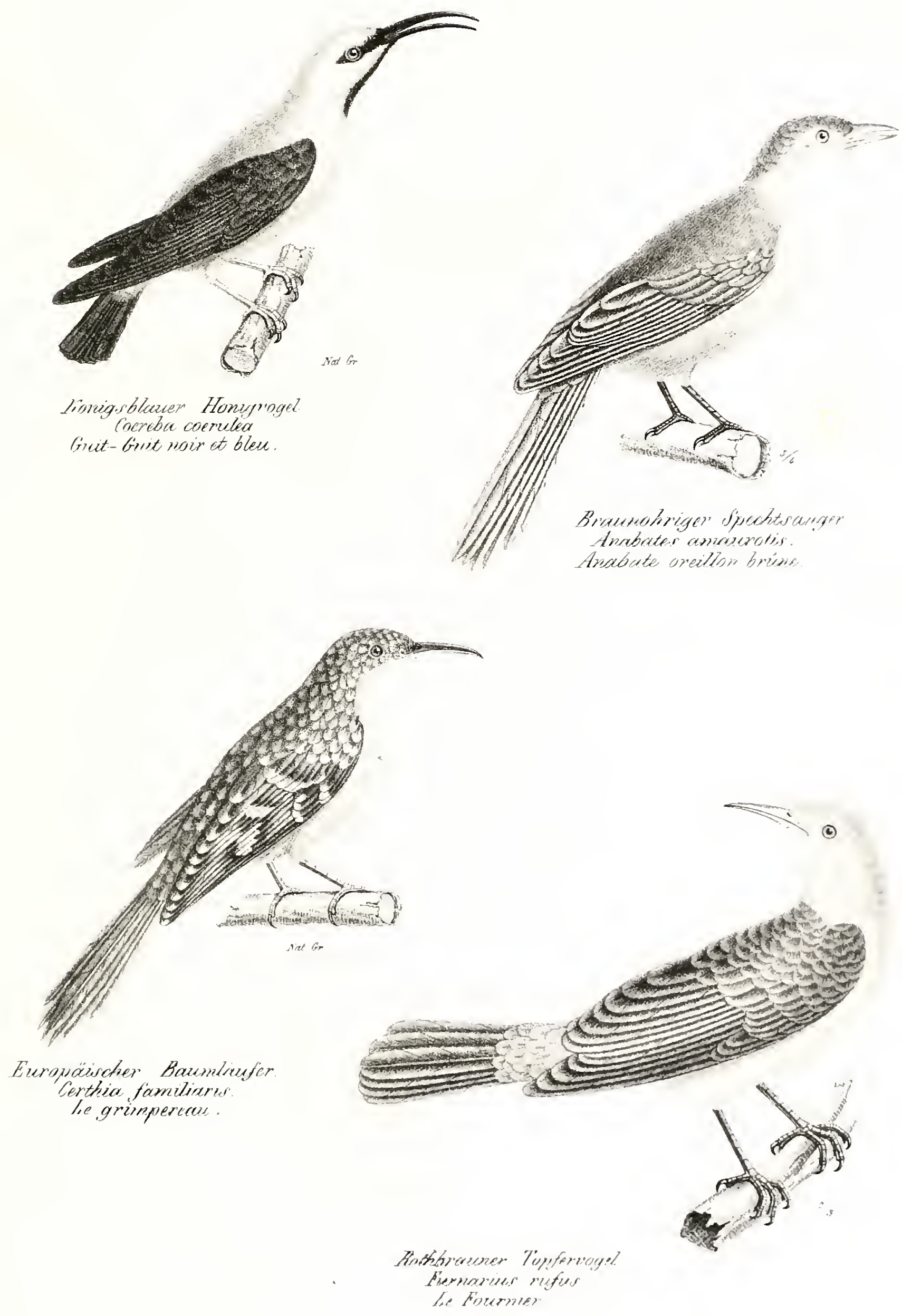


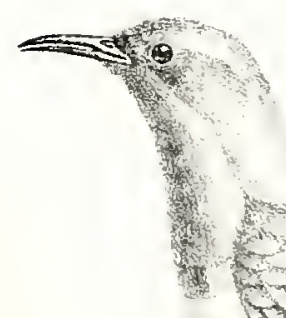

2

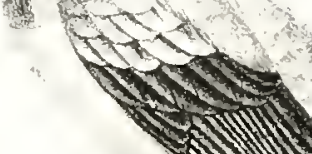

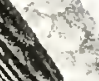
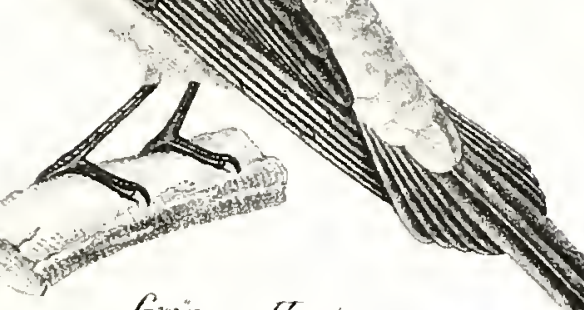

Gmizrer Honigsauger

Nectrivine metullece
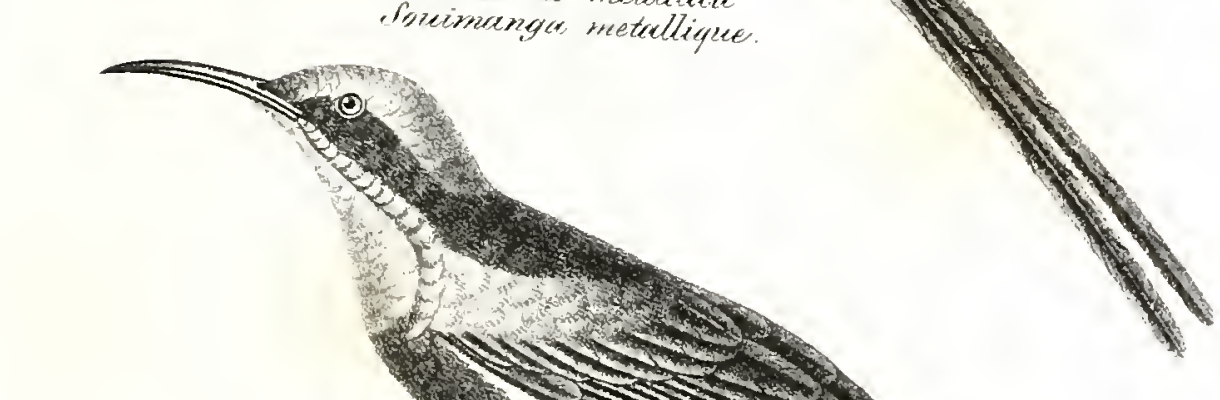

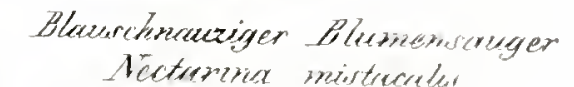
Solimange inoustre.

Risthbrüstiger. Lilumensunger.
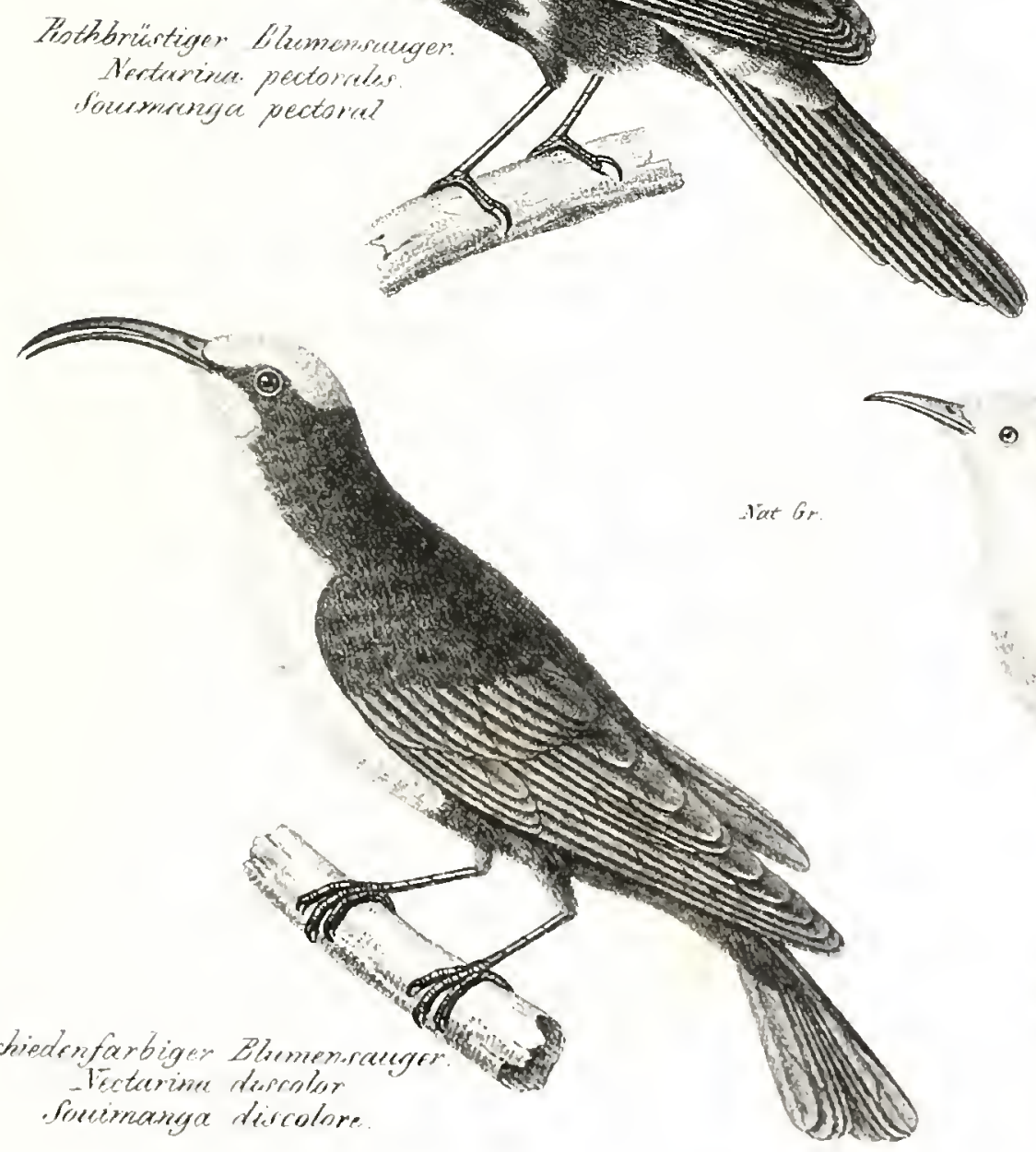

e

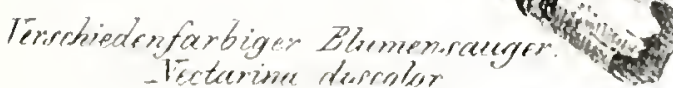
steeimaniga diecolore. 


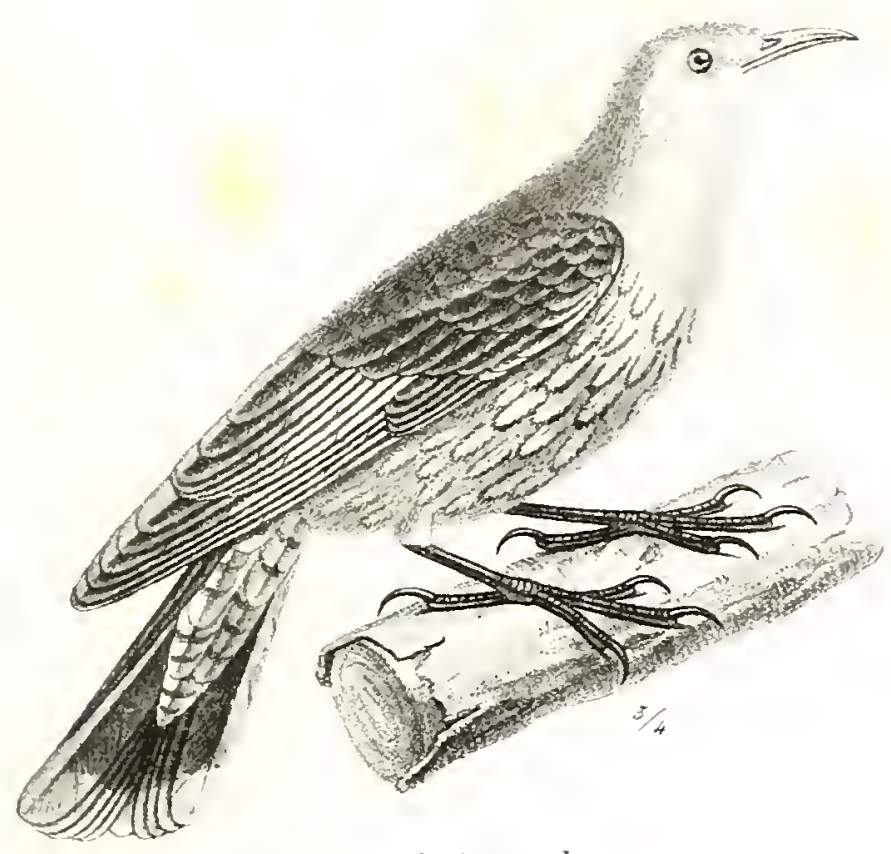

Spechtrutiger Steigvagel

Eschelet picumne

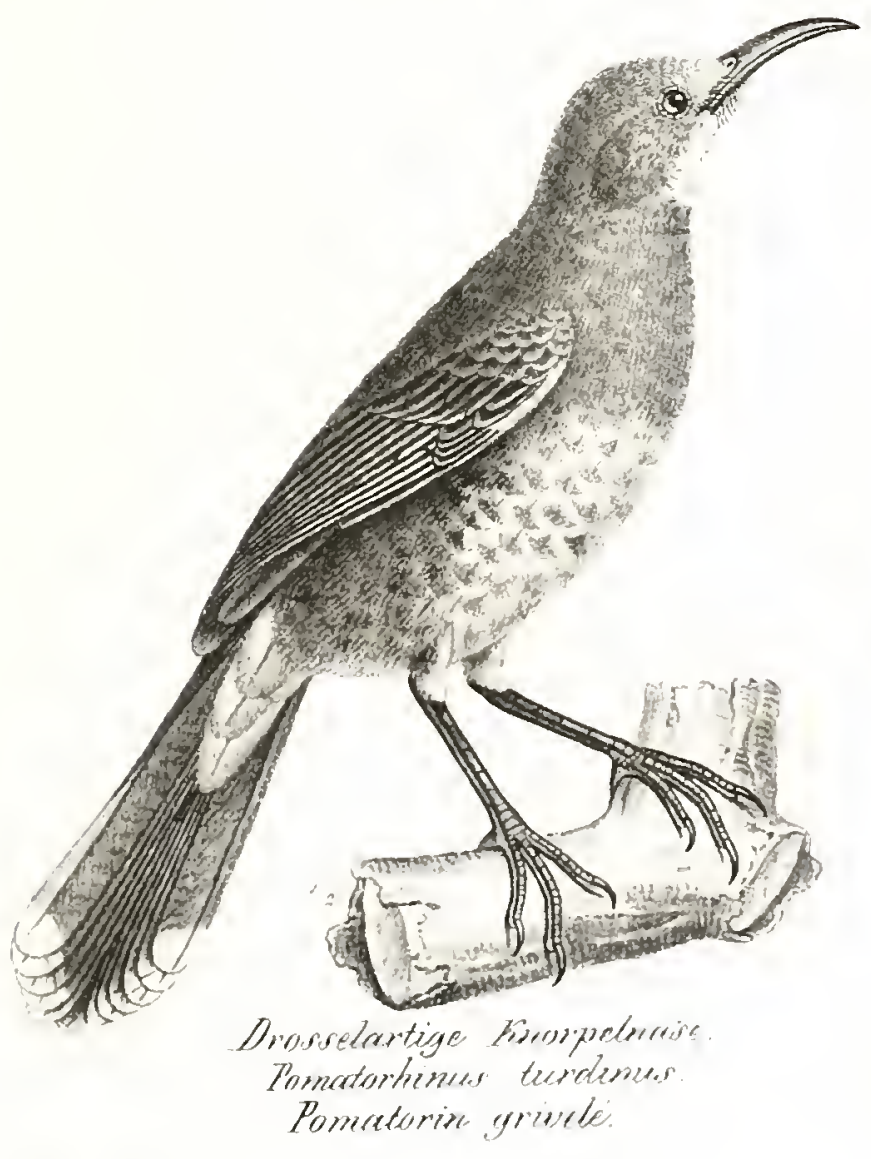

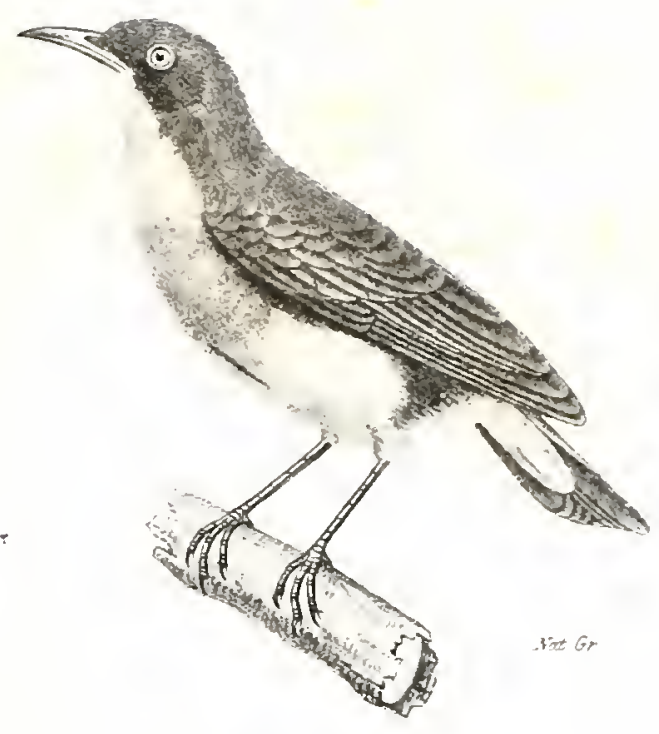

Blethrentiges Rothrogel Dicaurin werguimalenterm

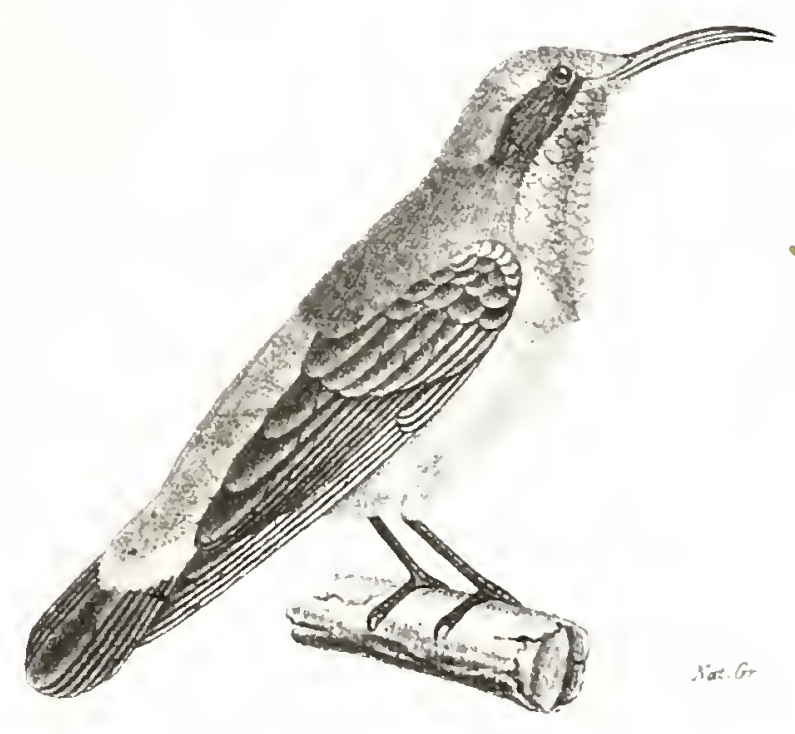

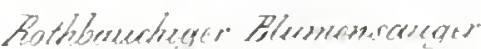

- lictominar coccisme

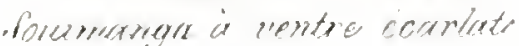




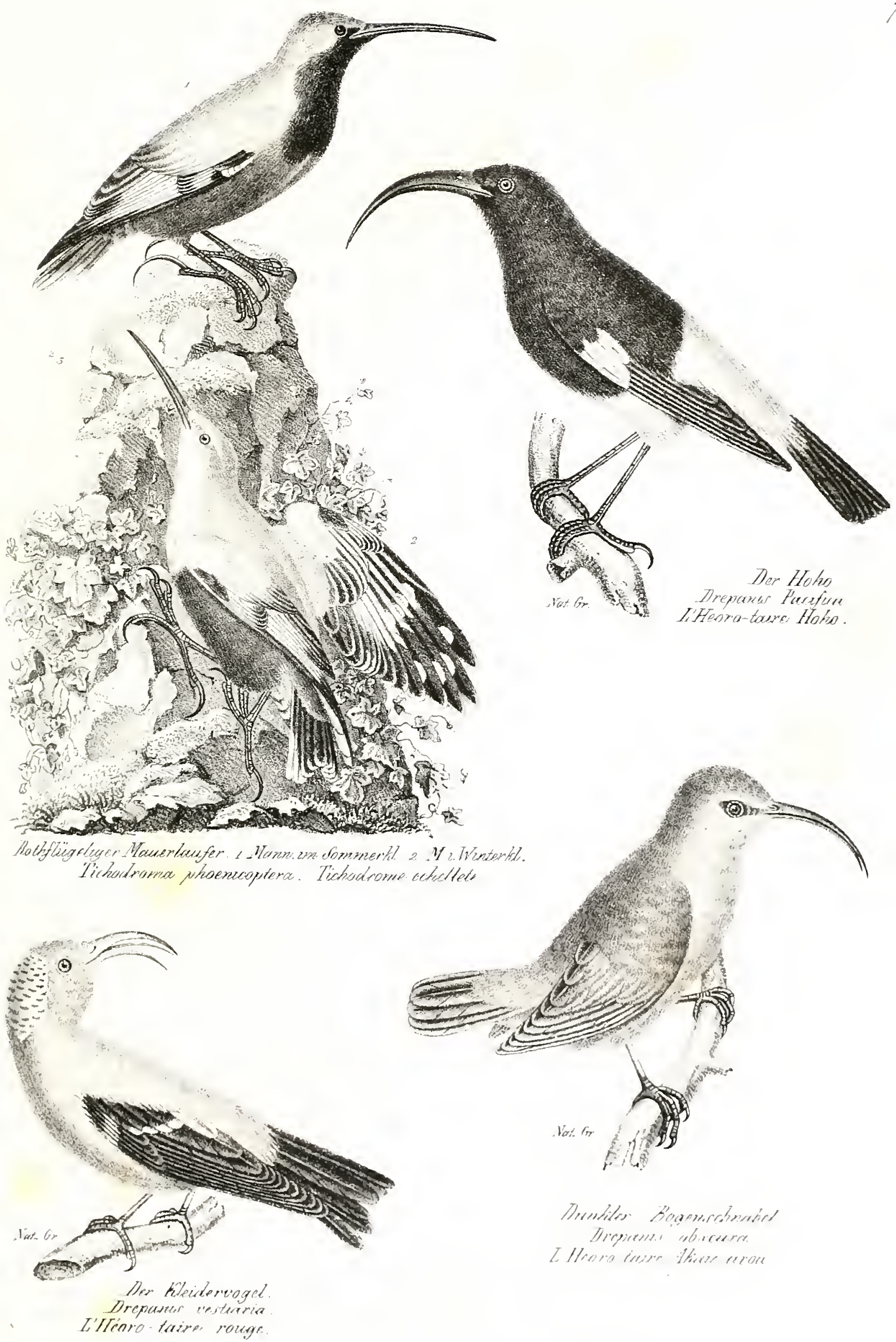




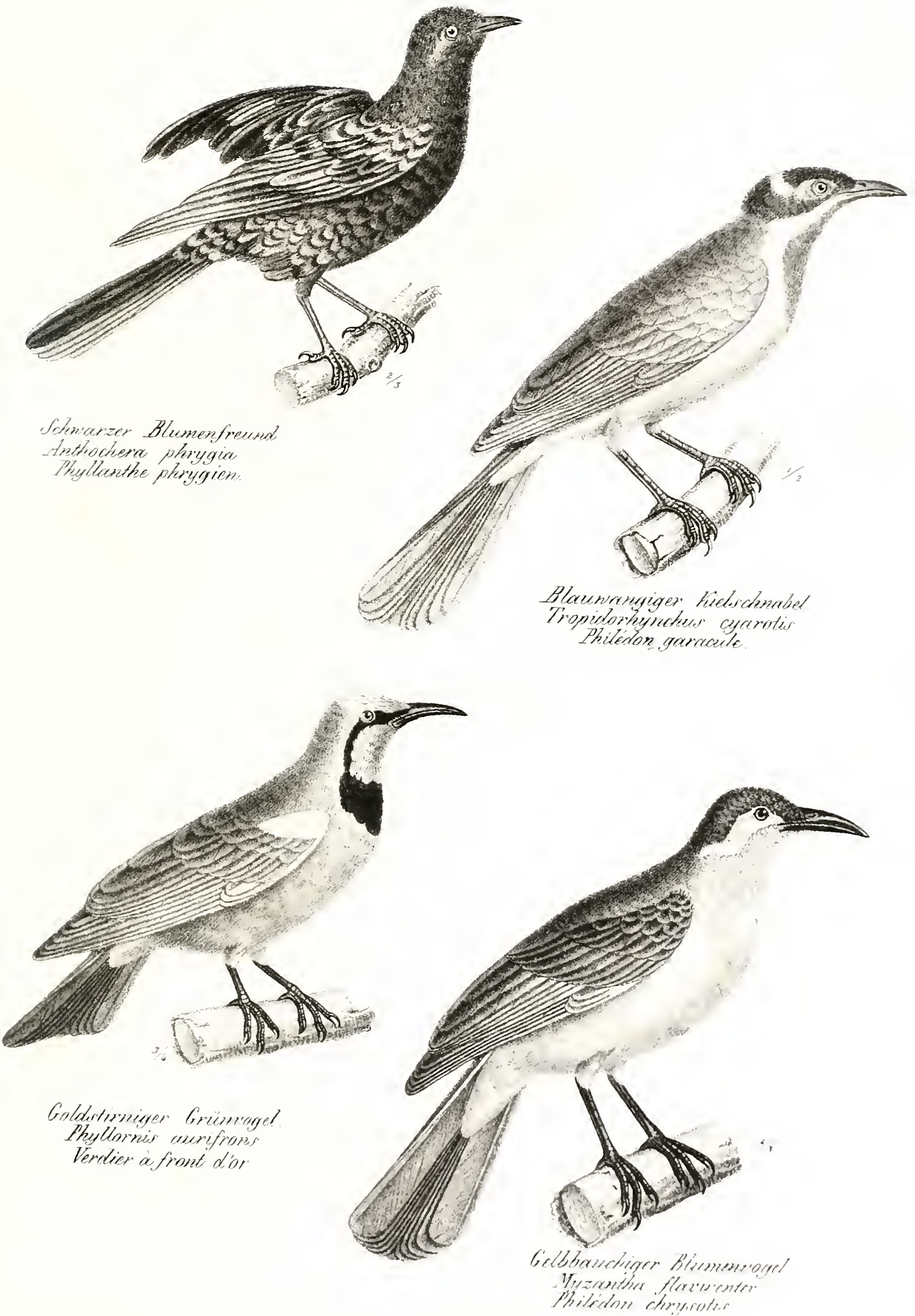


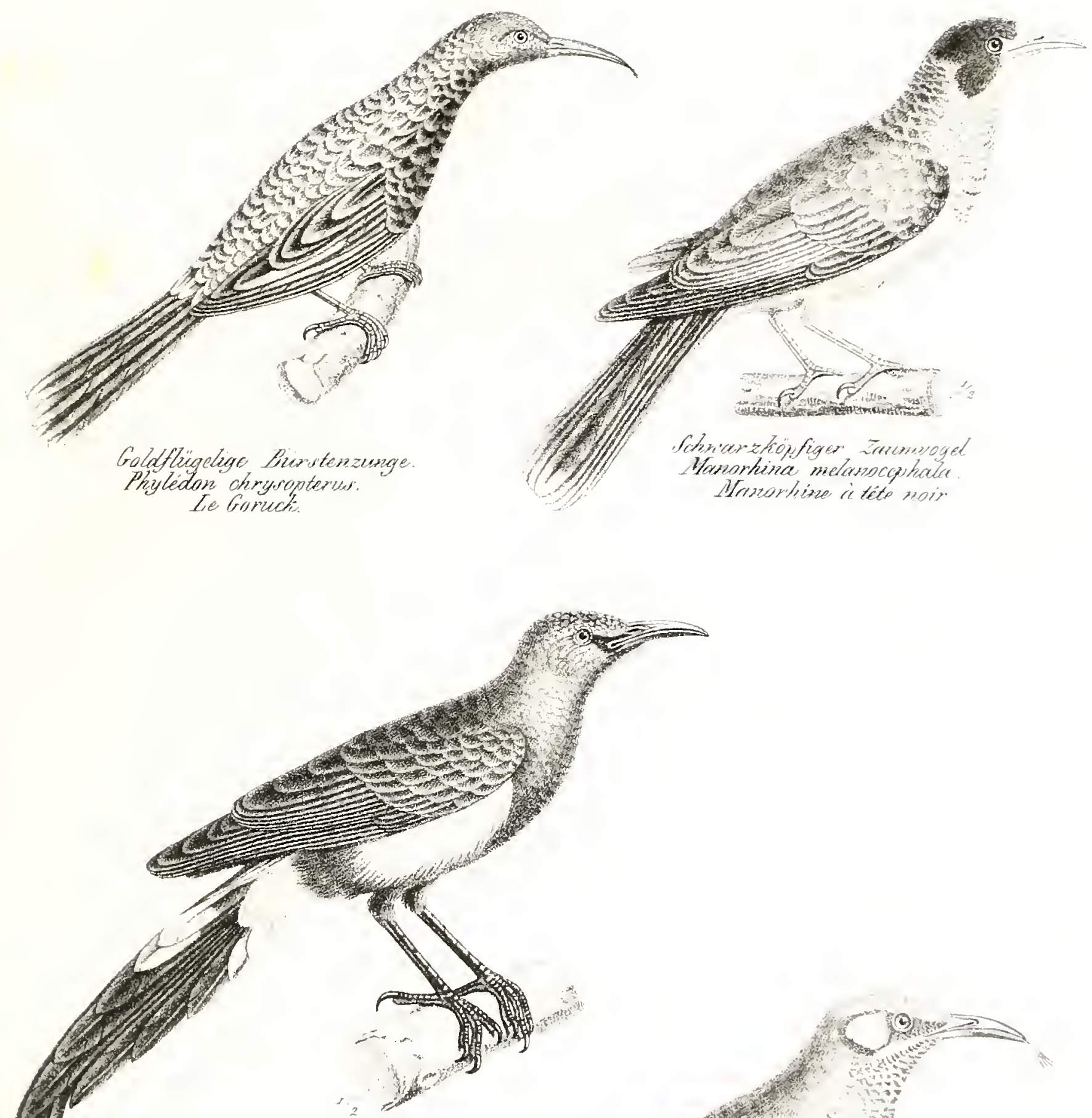

Des Whos

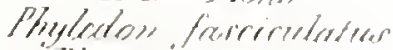

Phuglestan Wration

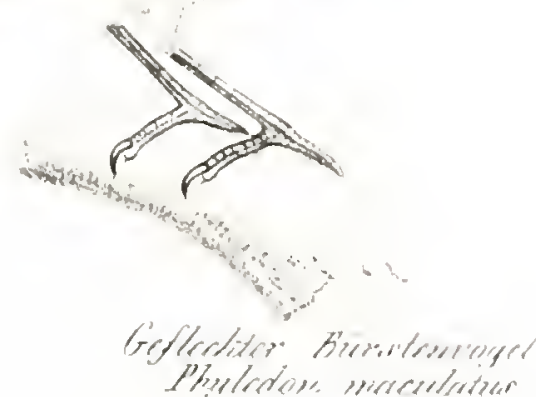

Phulfidon gritiele. 


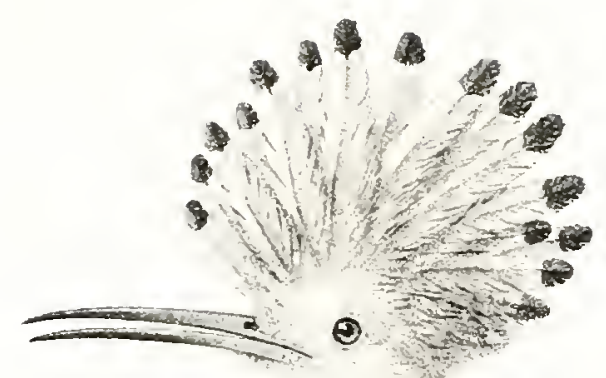

Europäischer Triedehopf. Upupa epopis
La Hispo.
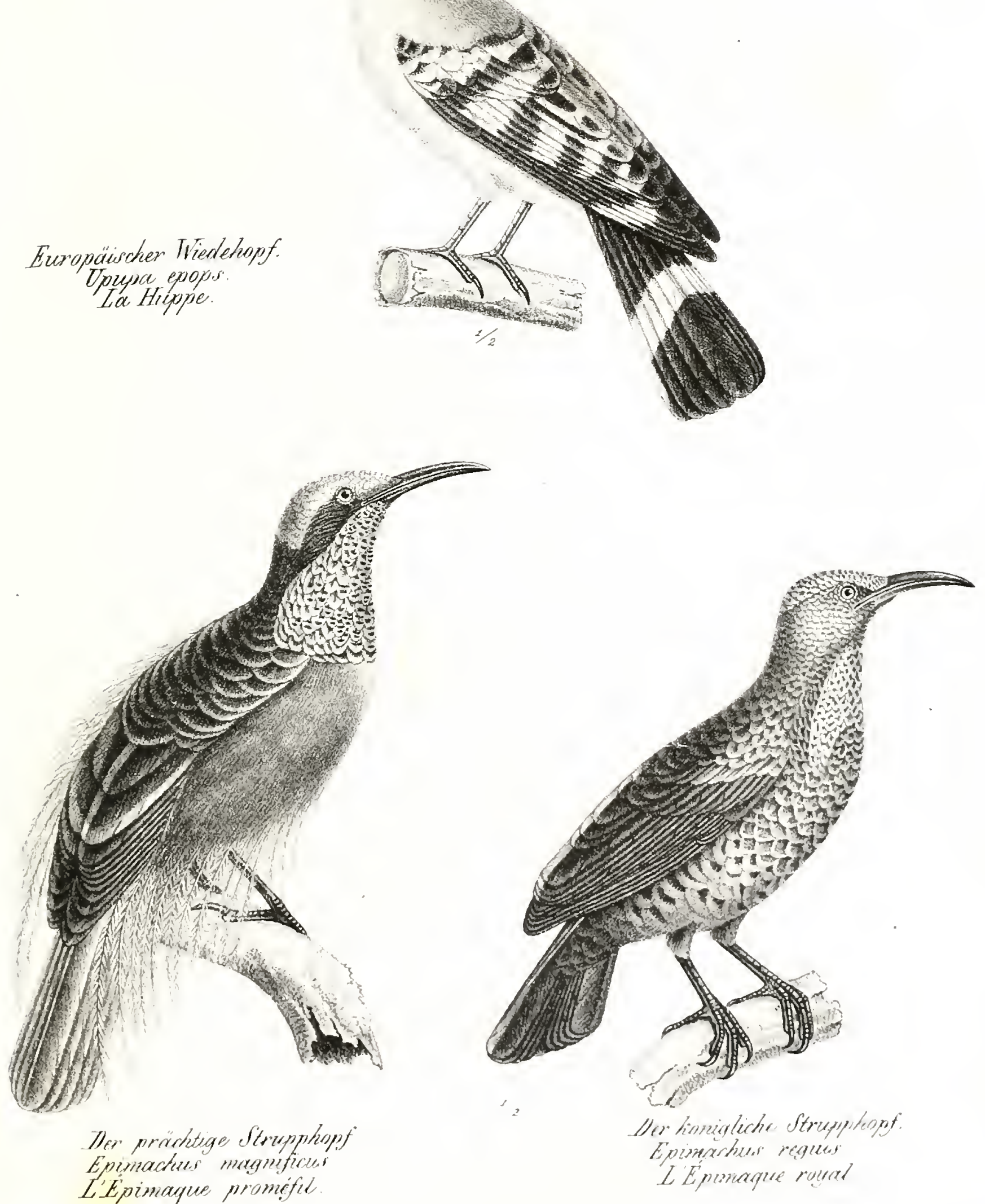


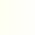



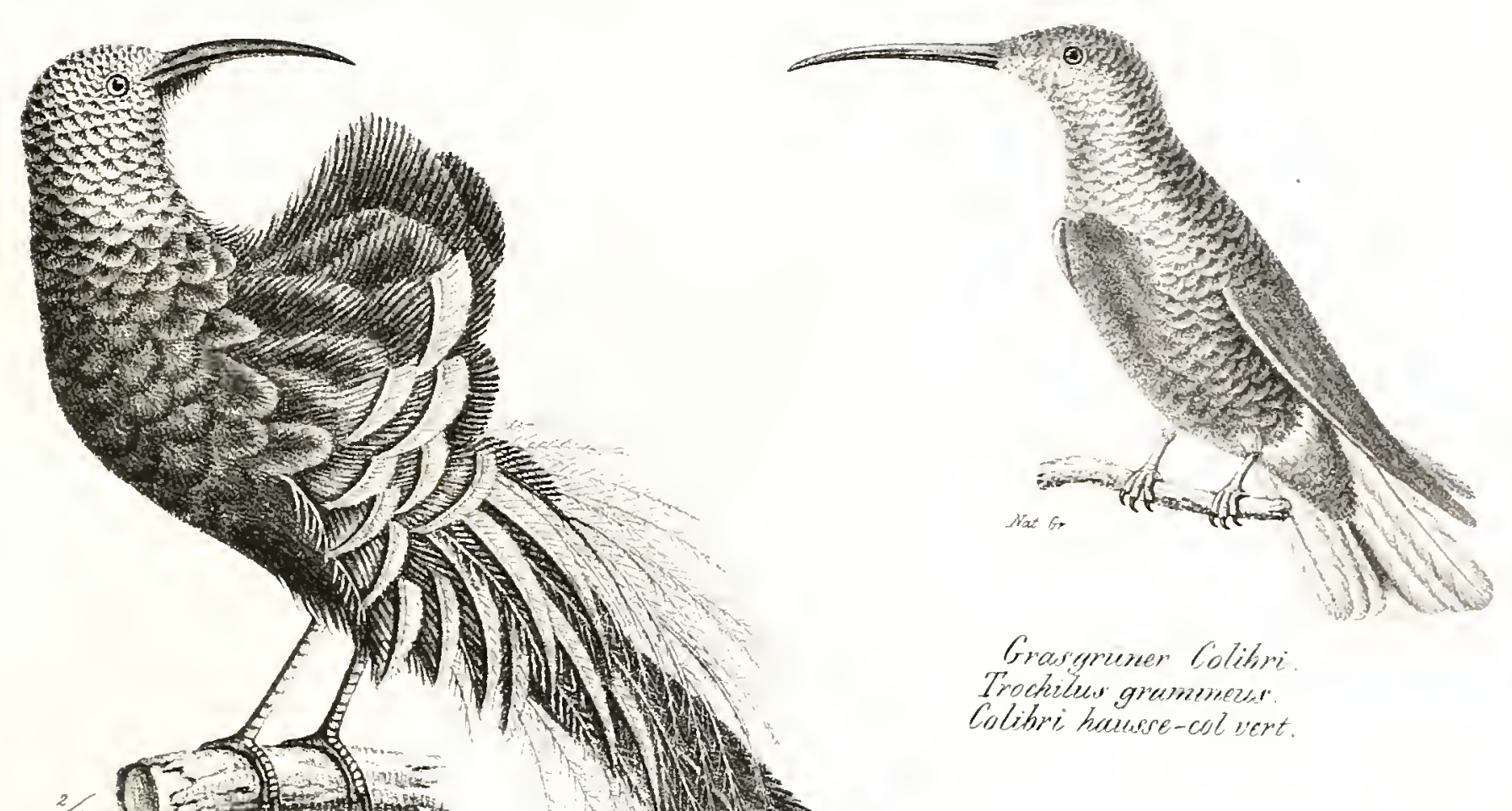

Der Stolze Strupphopf Eipimachus steperbus. Le grand promerops

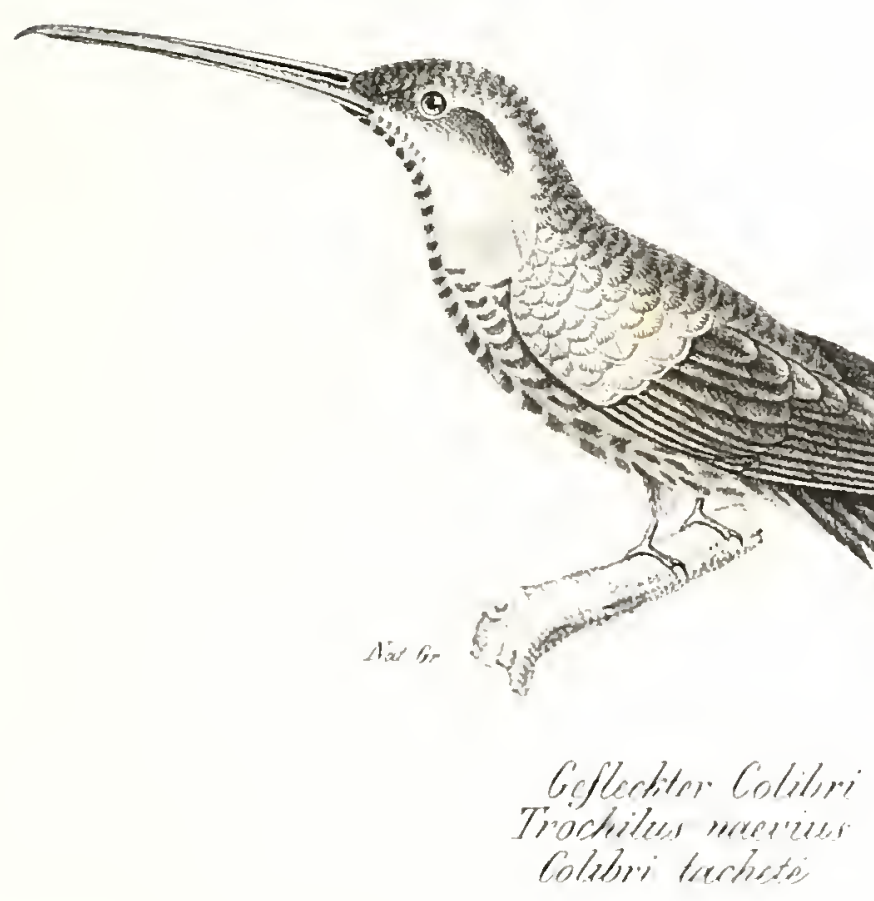





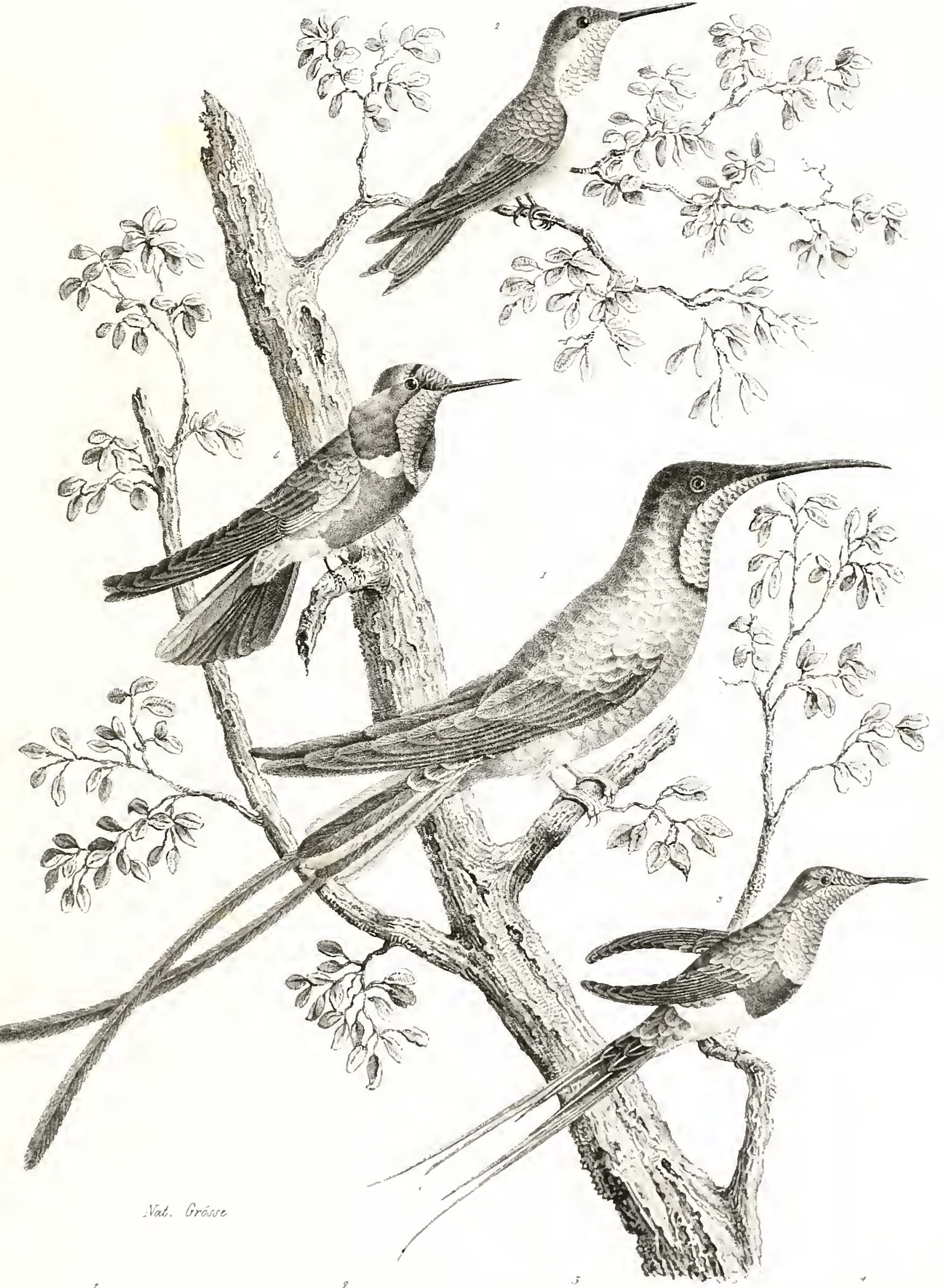

Topross-lialibiv. Trochiliw pellex. Golibri topaze.
Gemeiner Golibiri.

Trenshilate colubri, Discue-moushe melier

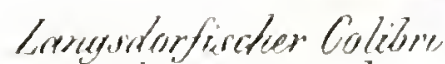
Irochiters Laugudorfie

Gesrbelderter Ciolition Trochilues" sculater.

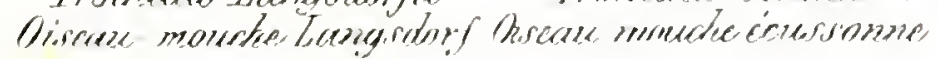





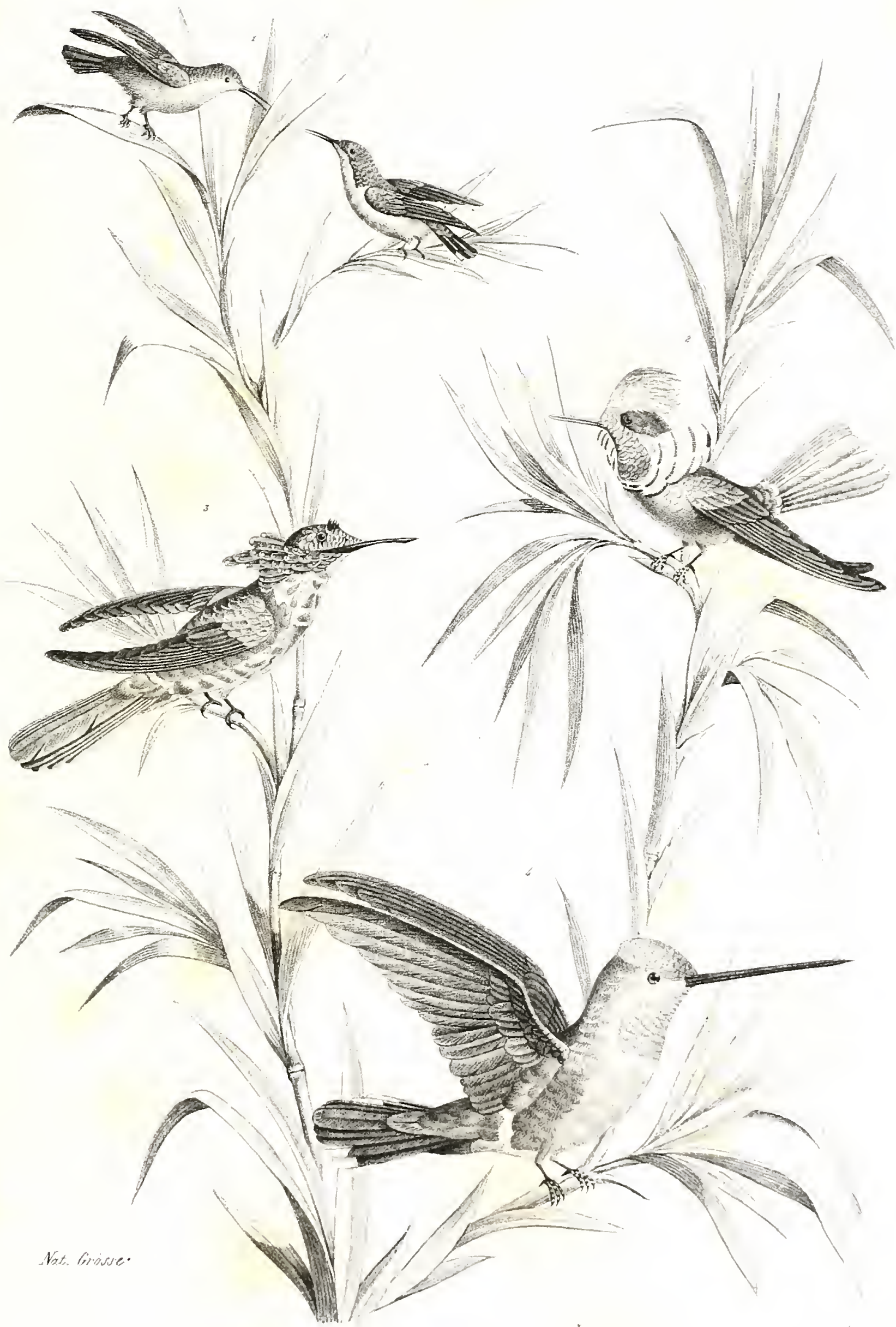

Kleinster Colutori.

Trochilens minumisis.

Pröstitiger Colibri Trochitus morsmificus.

Itubhiglanzender. Coltibri Ptolase Cioliber Tirnchilue chalebreses

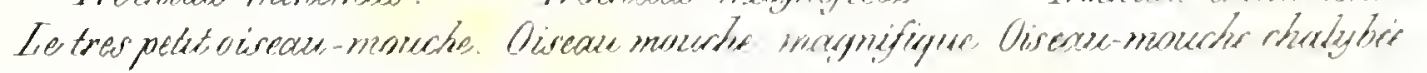

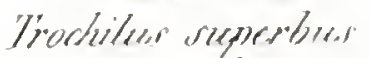

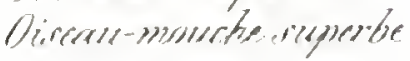




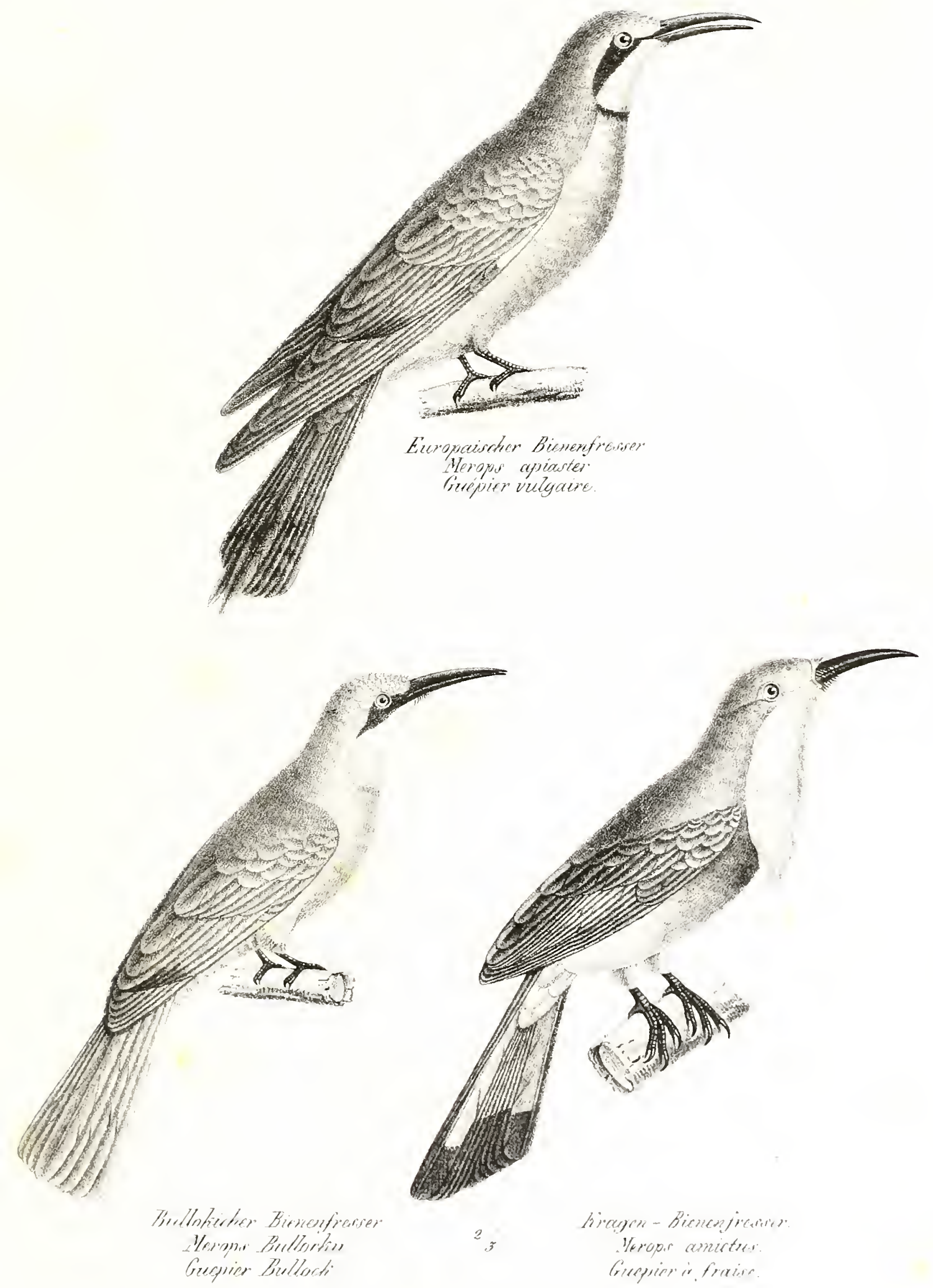


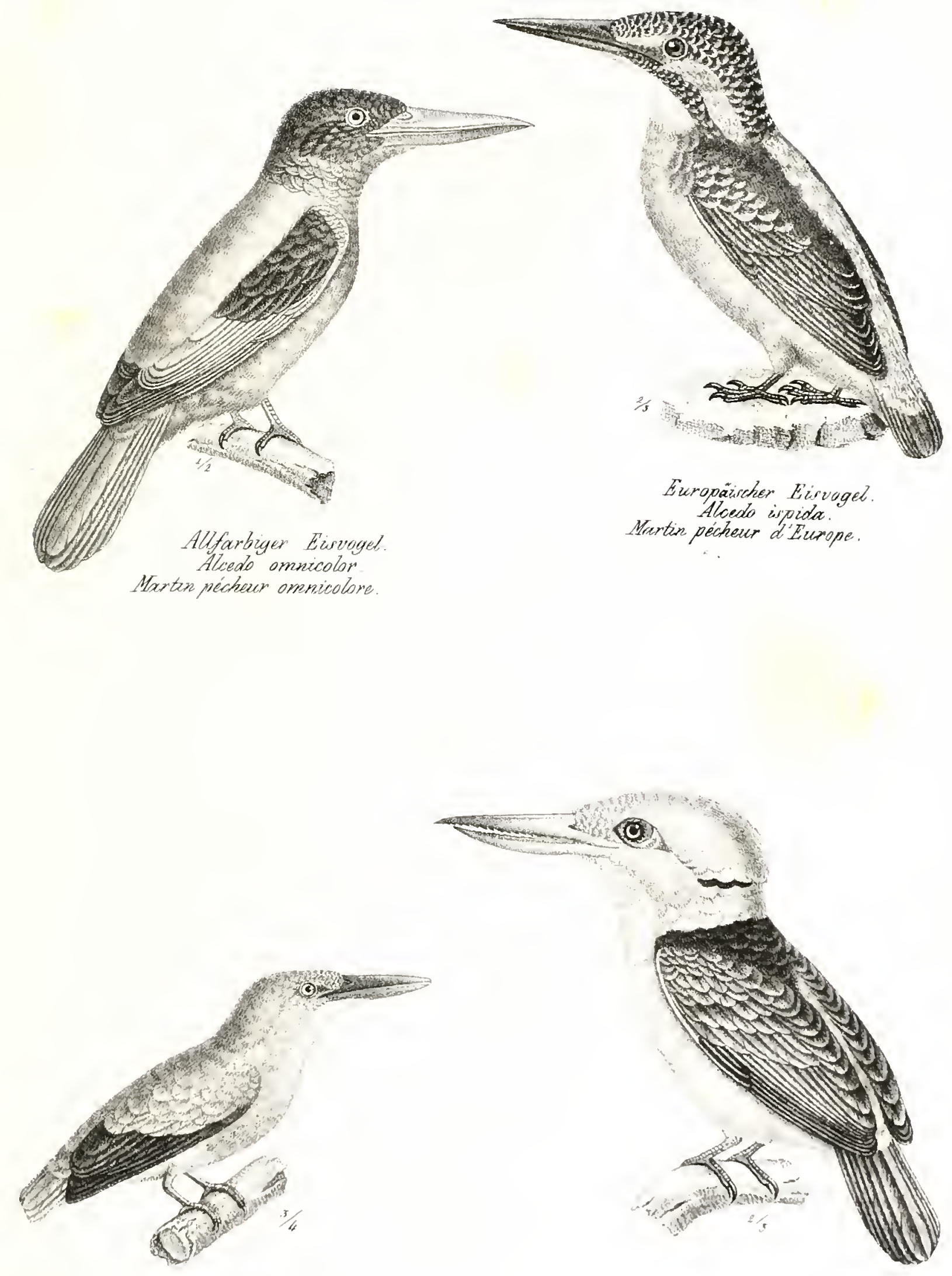

$$
\begin{aligned}
& \text { Dreizchigrer Ritiruaget } \\
& \text { Alcedo liesne } \\
& \text { Arion Ceysir. }
\end{aligned}
$$




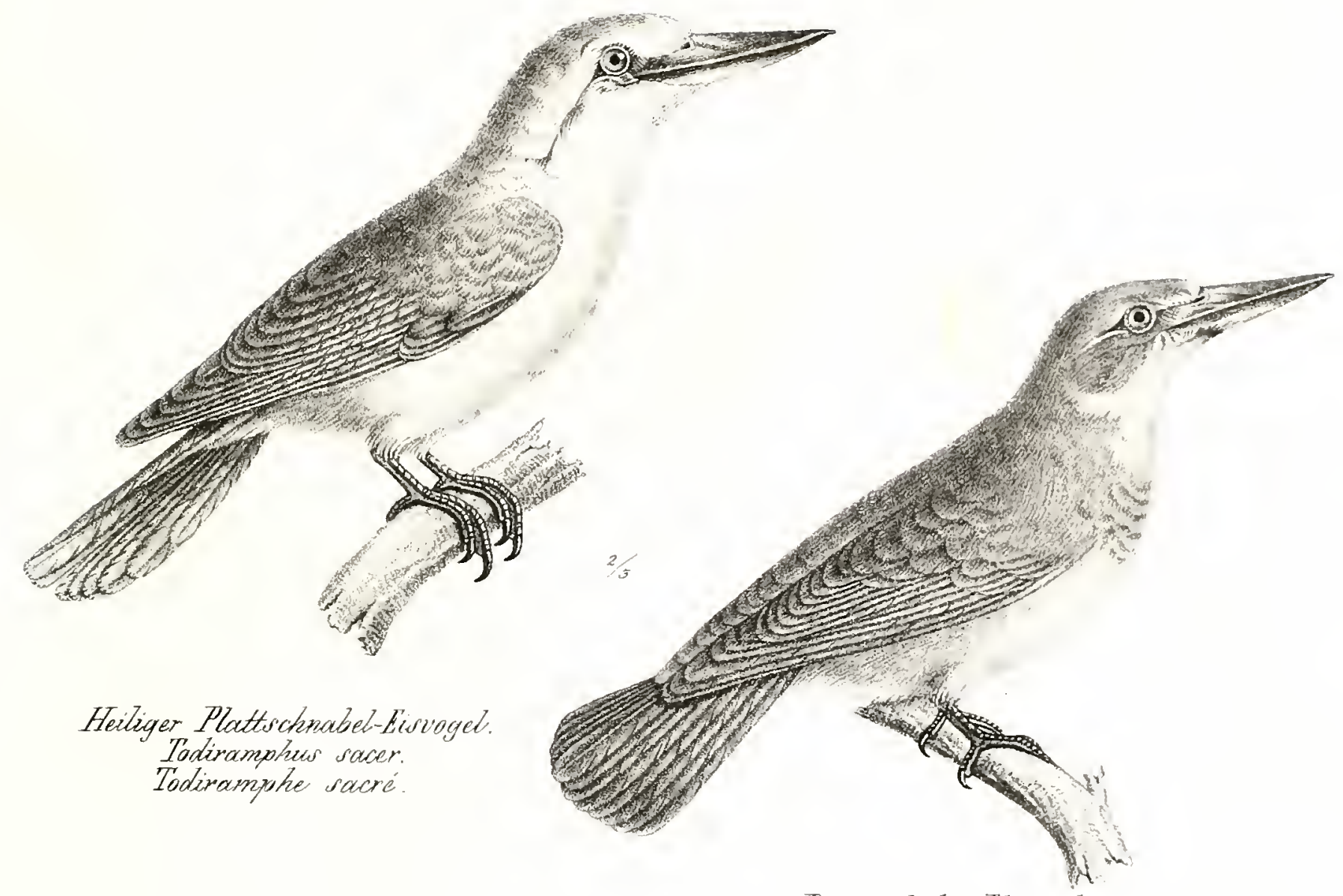

Der youttiche. Plattischmabel-Eisviagel. Todiratmphes. diveraws Todiramphe Dieu

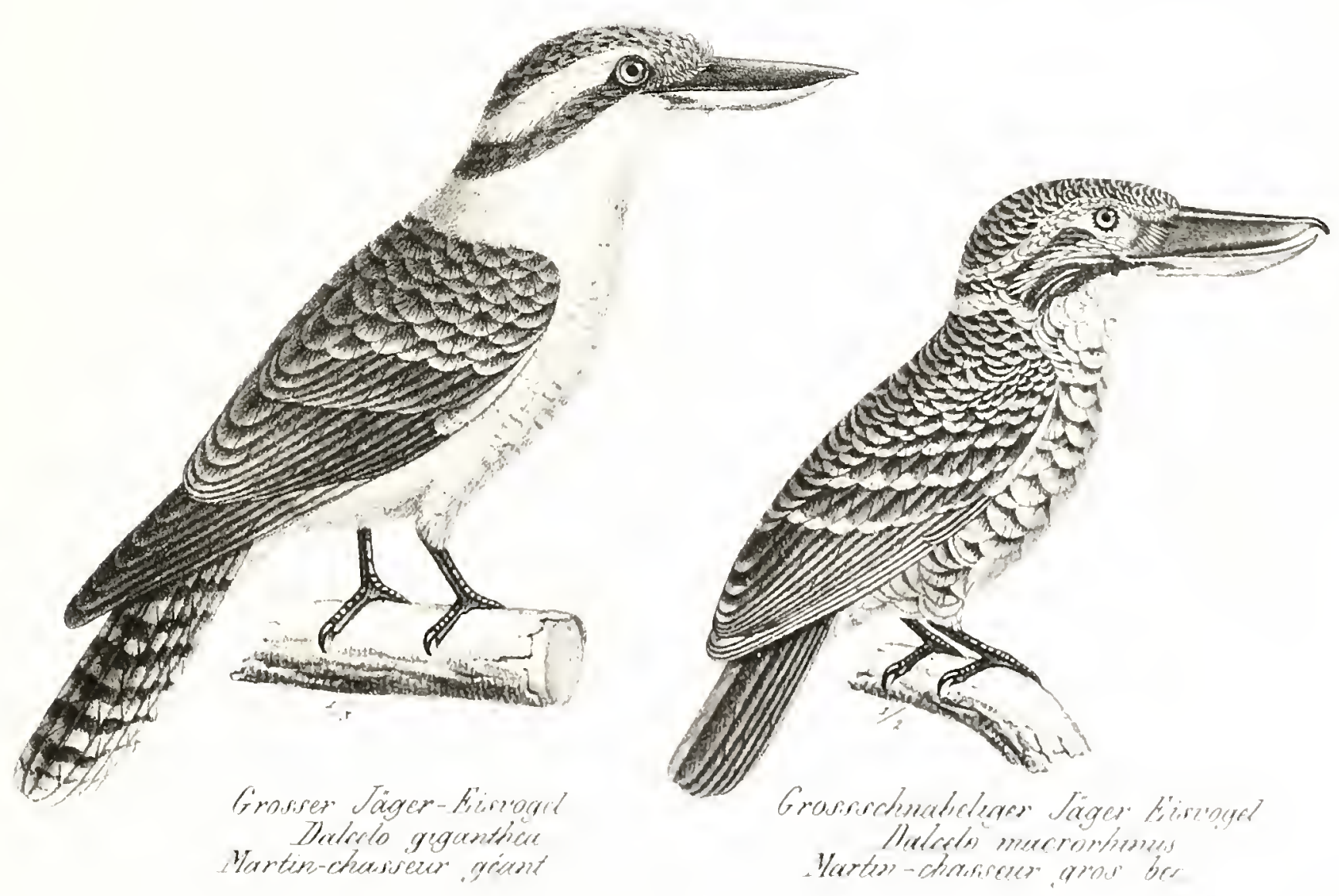




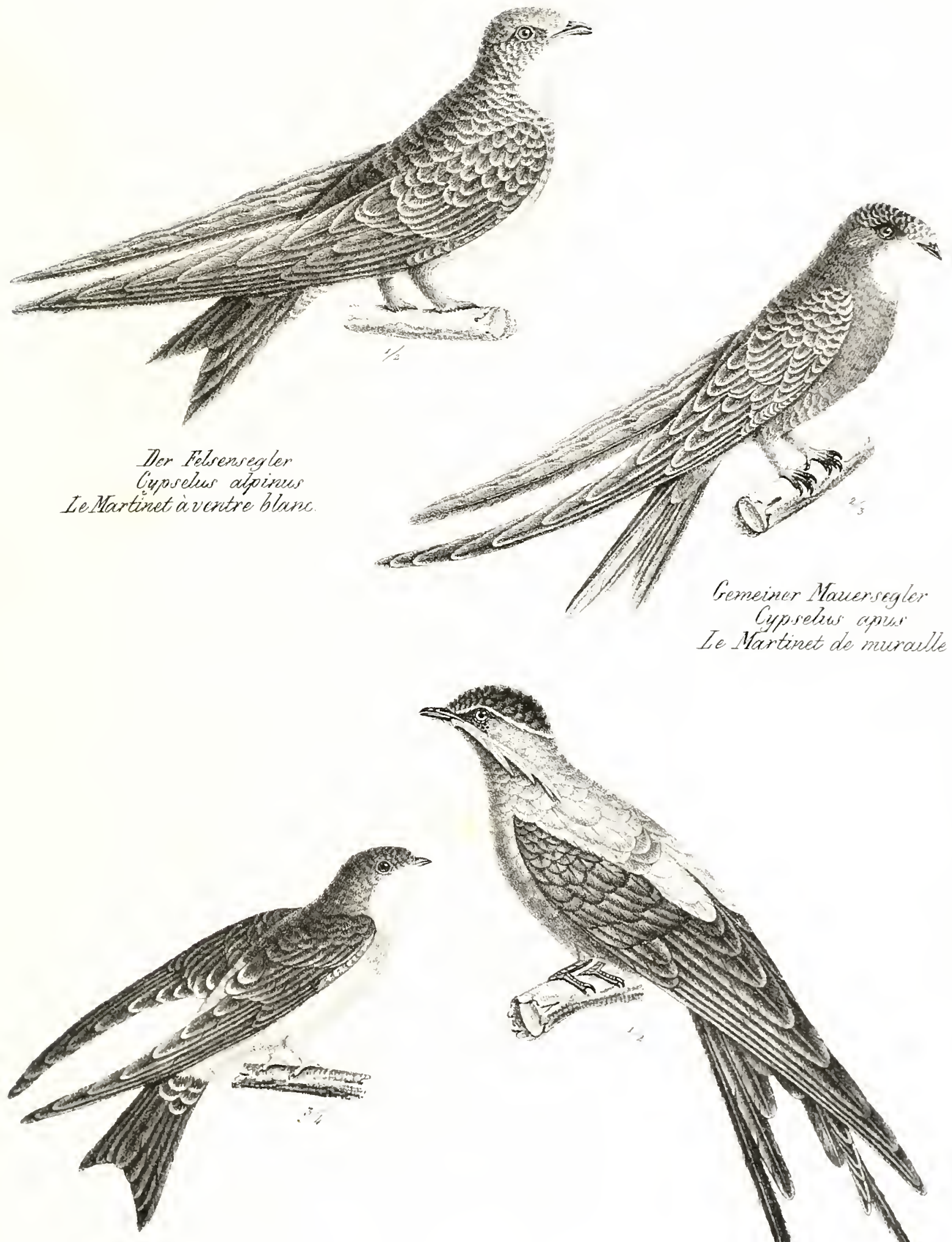

Die Haussotenialbe

thevesulo urbica

Heromdello de fenstere

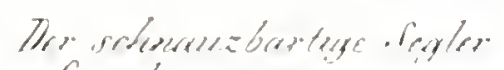
Cyporders msintrecence.

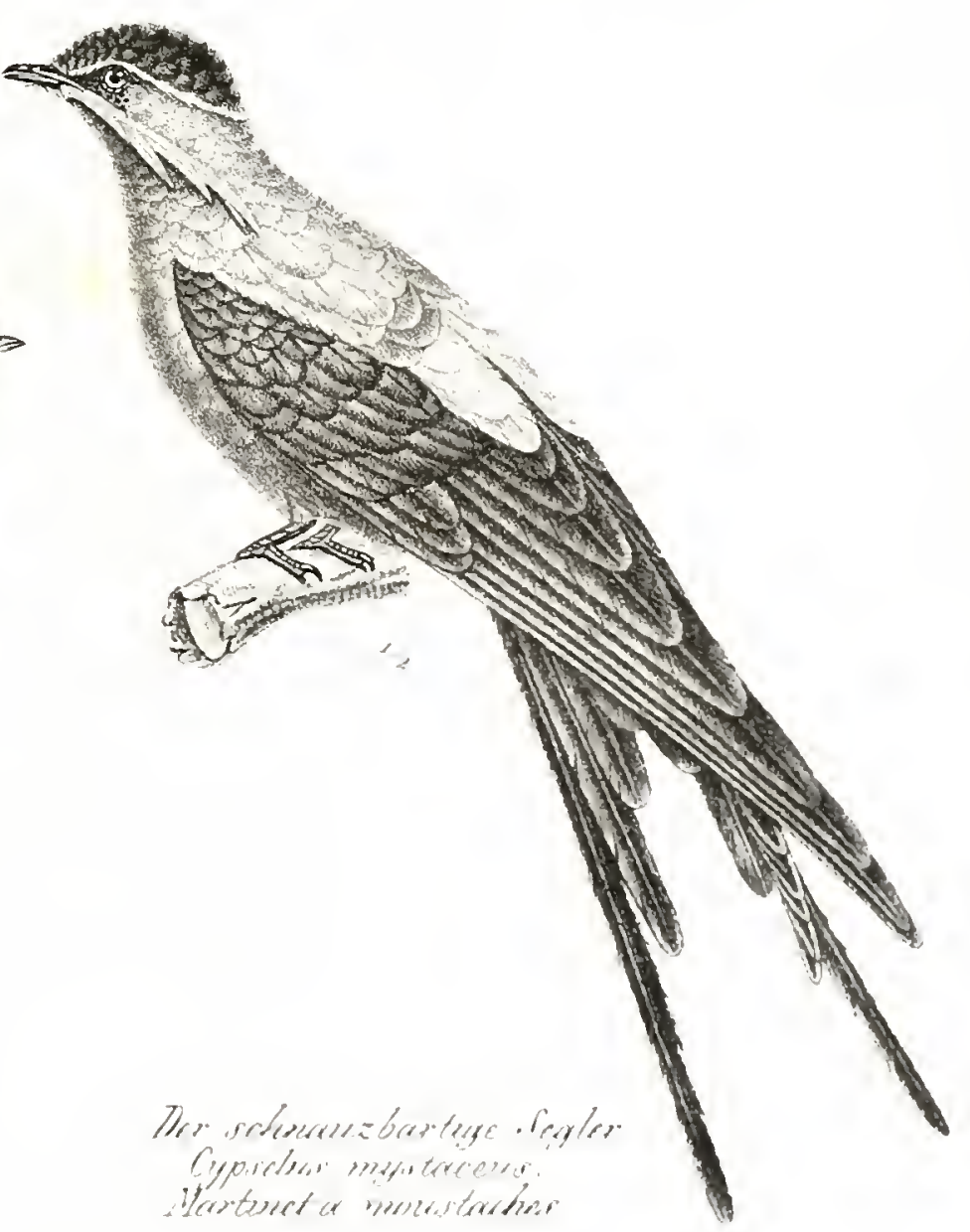





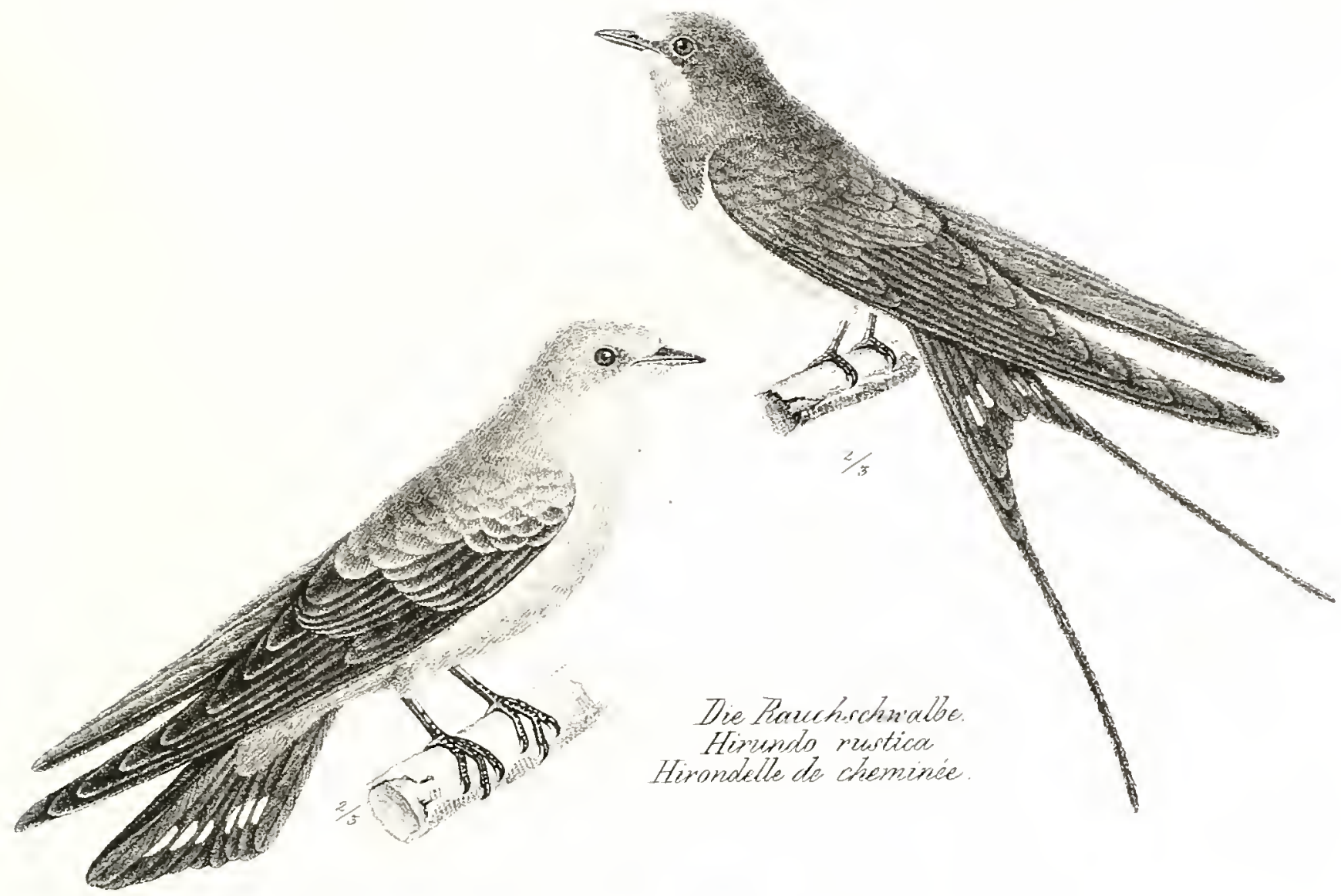

Die Felsenschnoulbe Hirundo reppestris. Hirnadelle de rocher

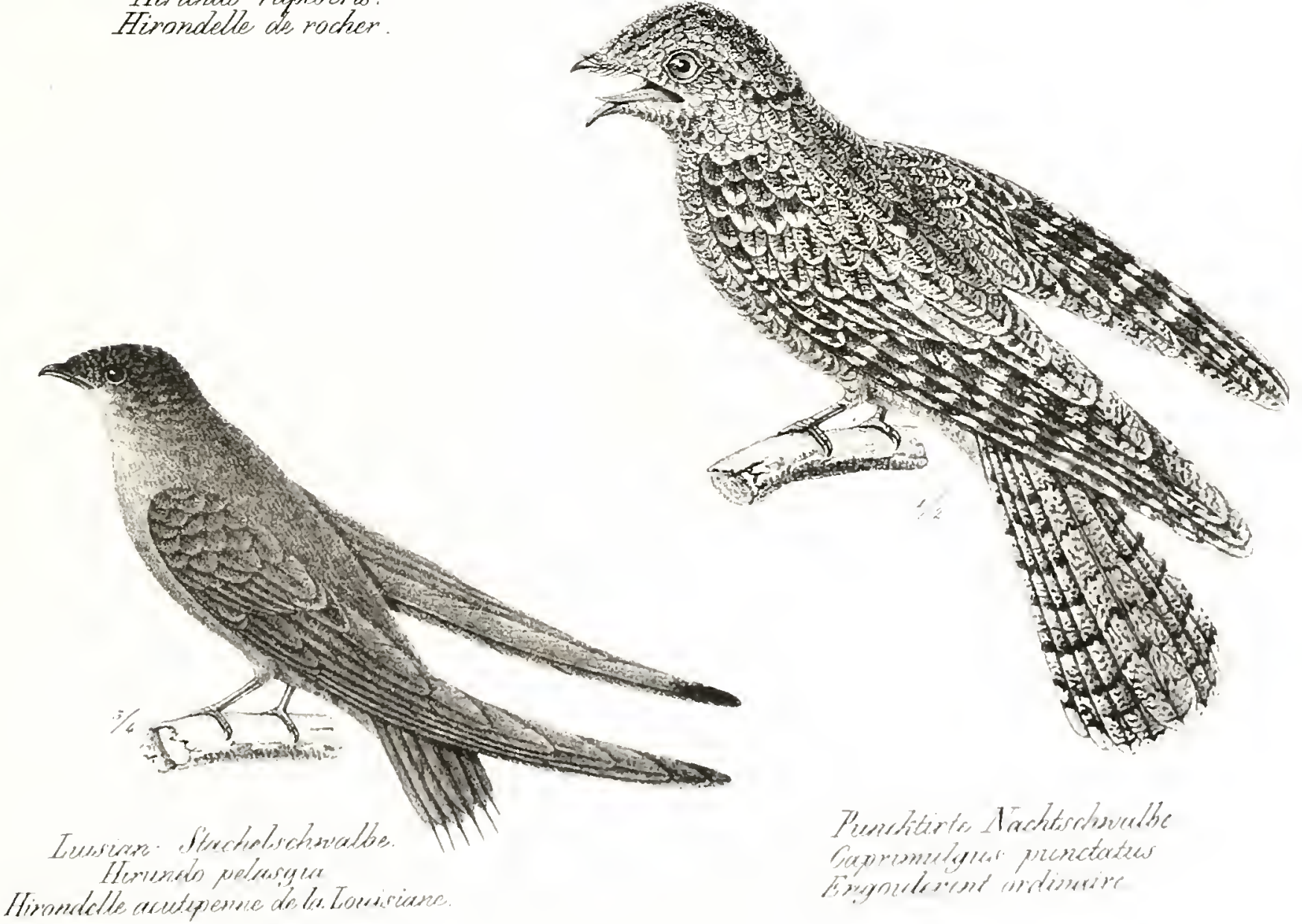




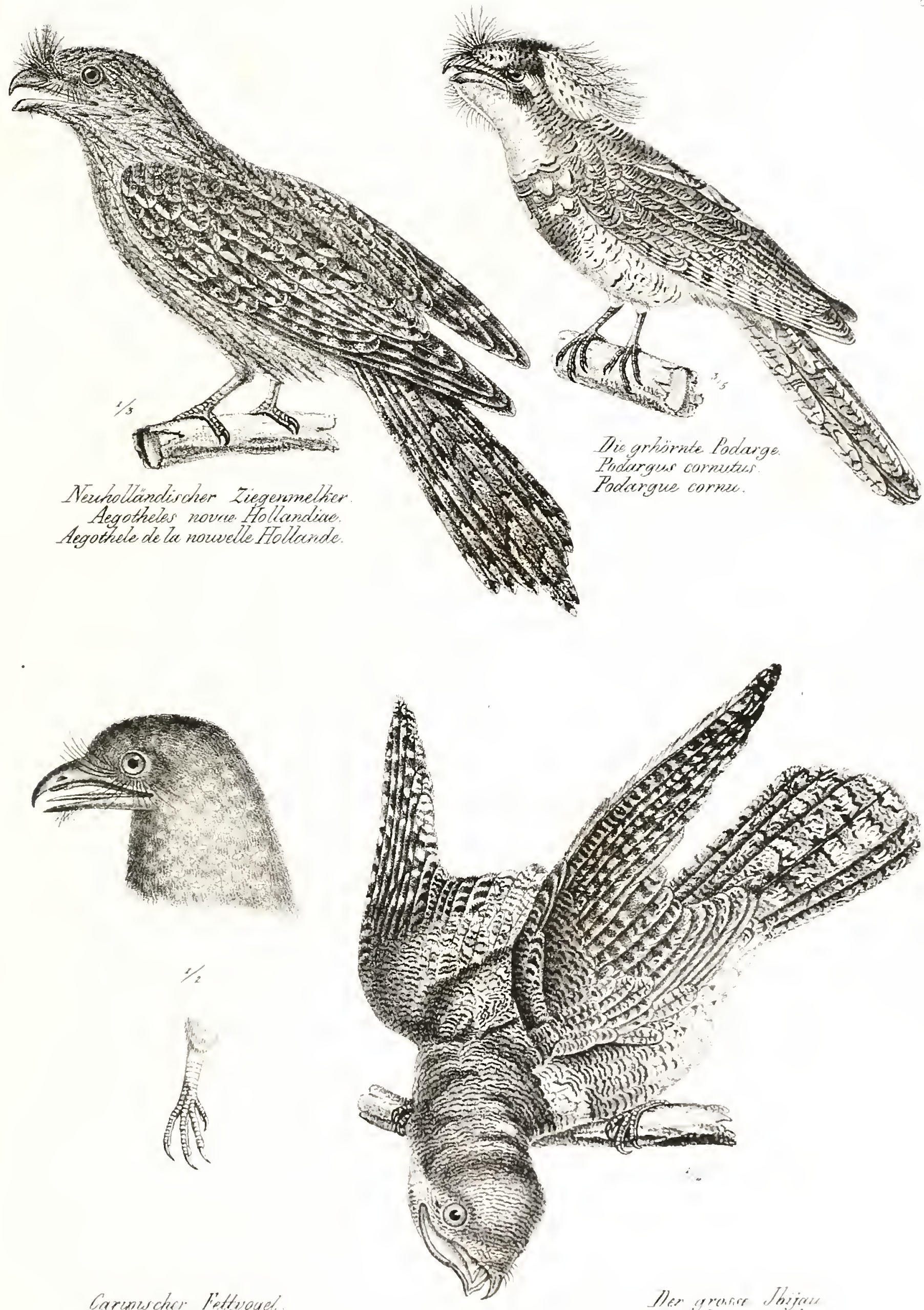

Carposcher liettroyed.

Stcatomis corincisis

- Tigrizhuser arromilio Le Guevenuro 



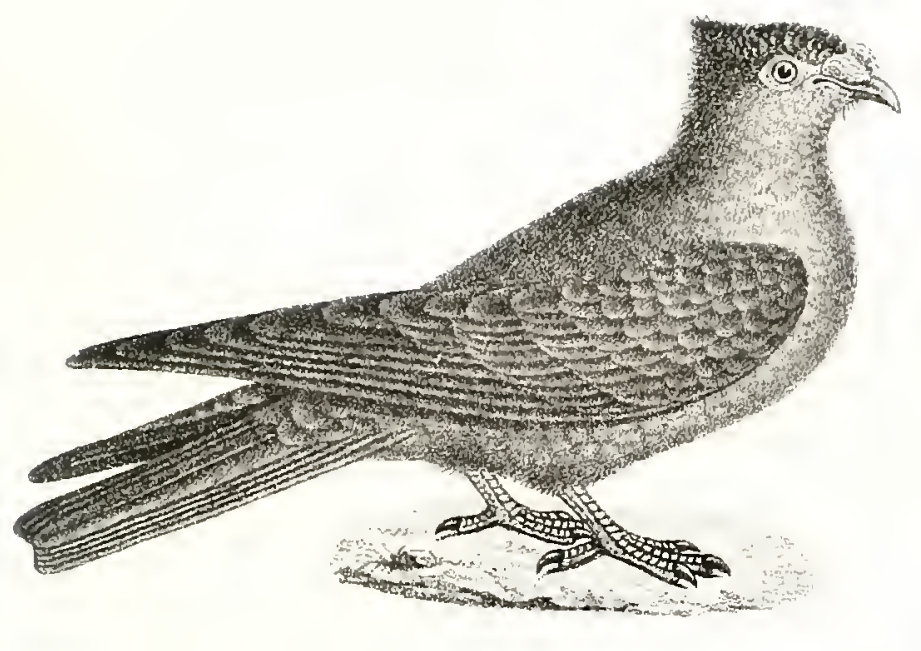

Die turficische Taube. 's Columber turcice. Pigeon Tinc.
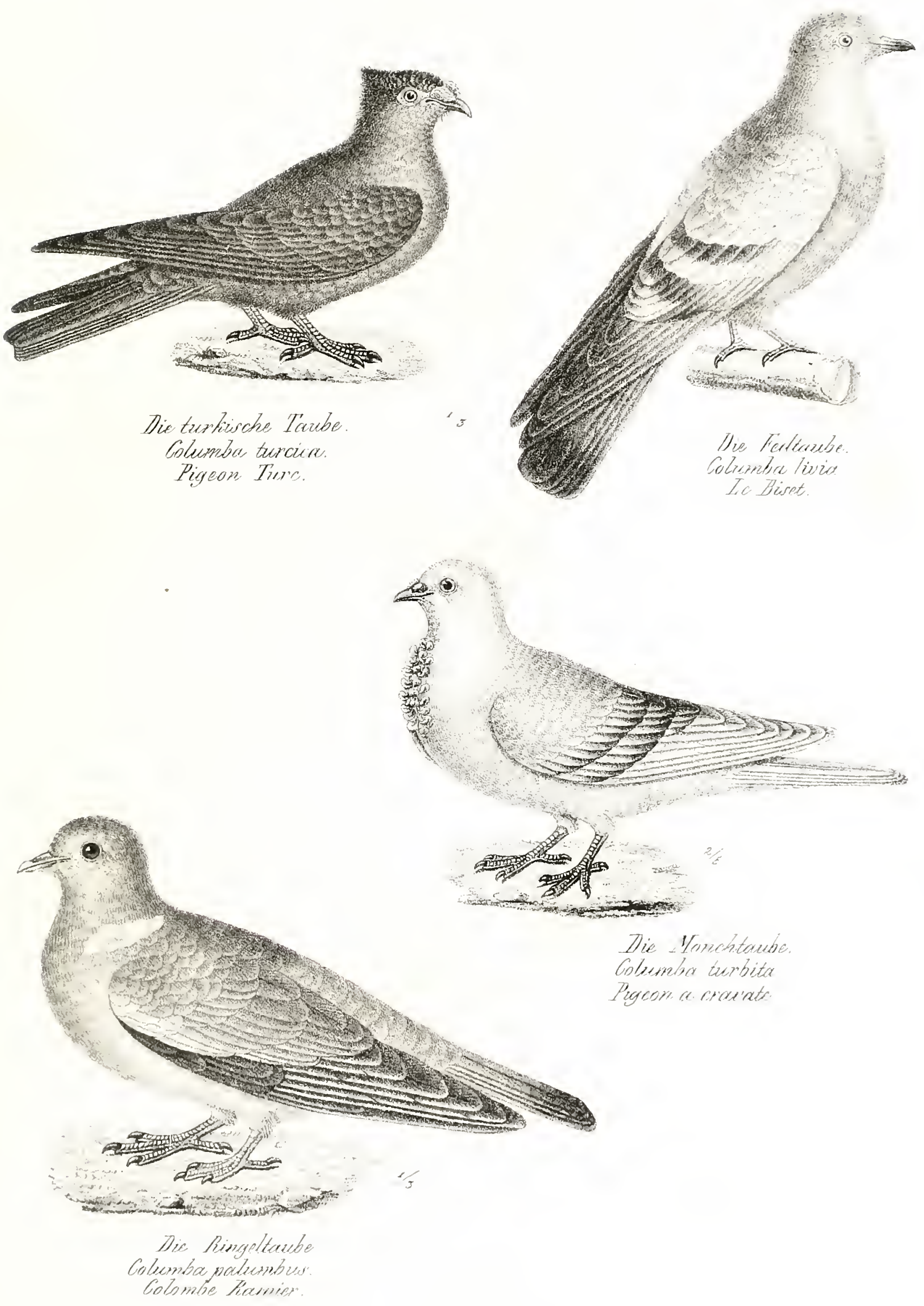


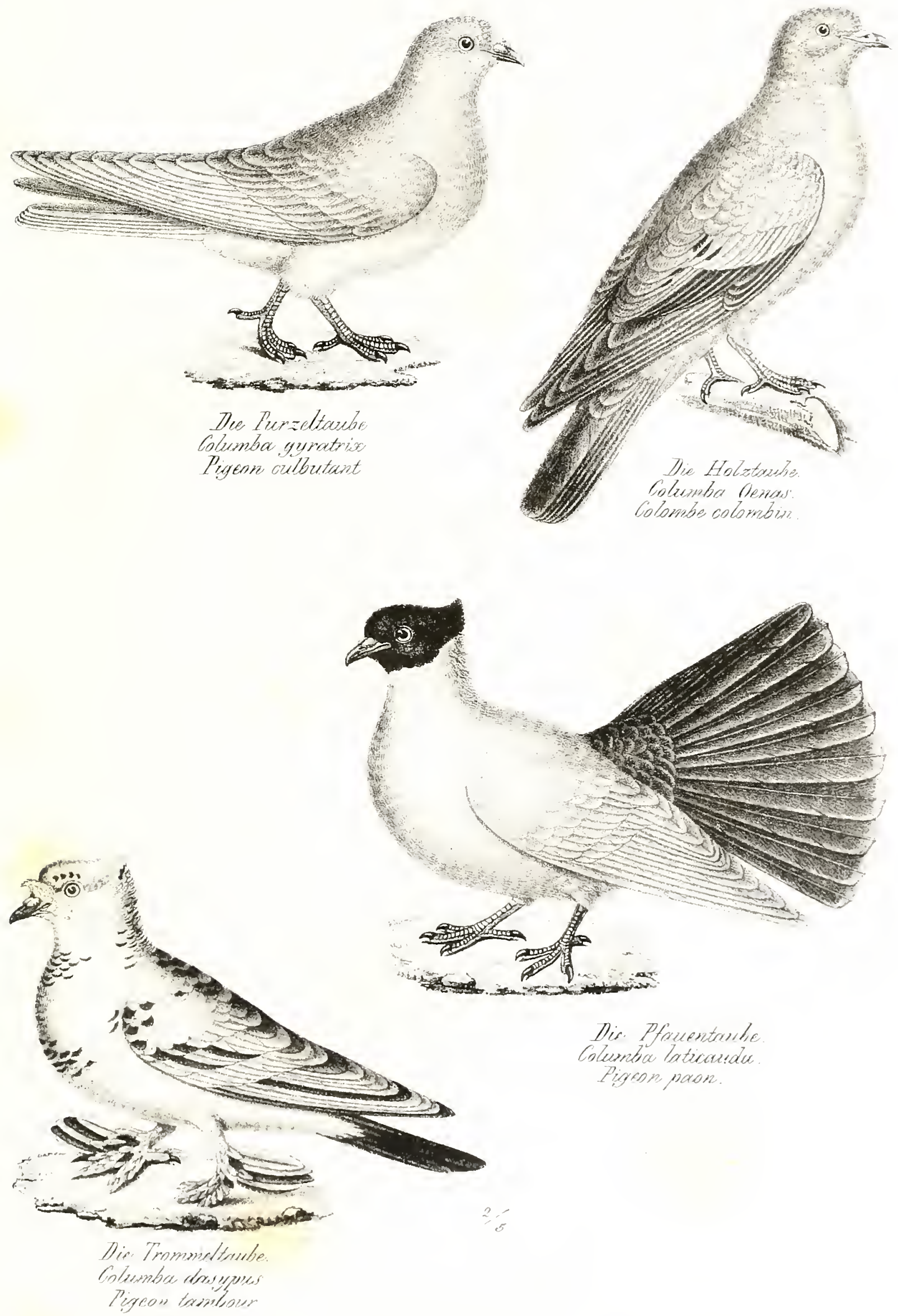

(c) $\tan$
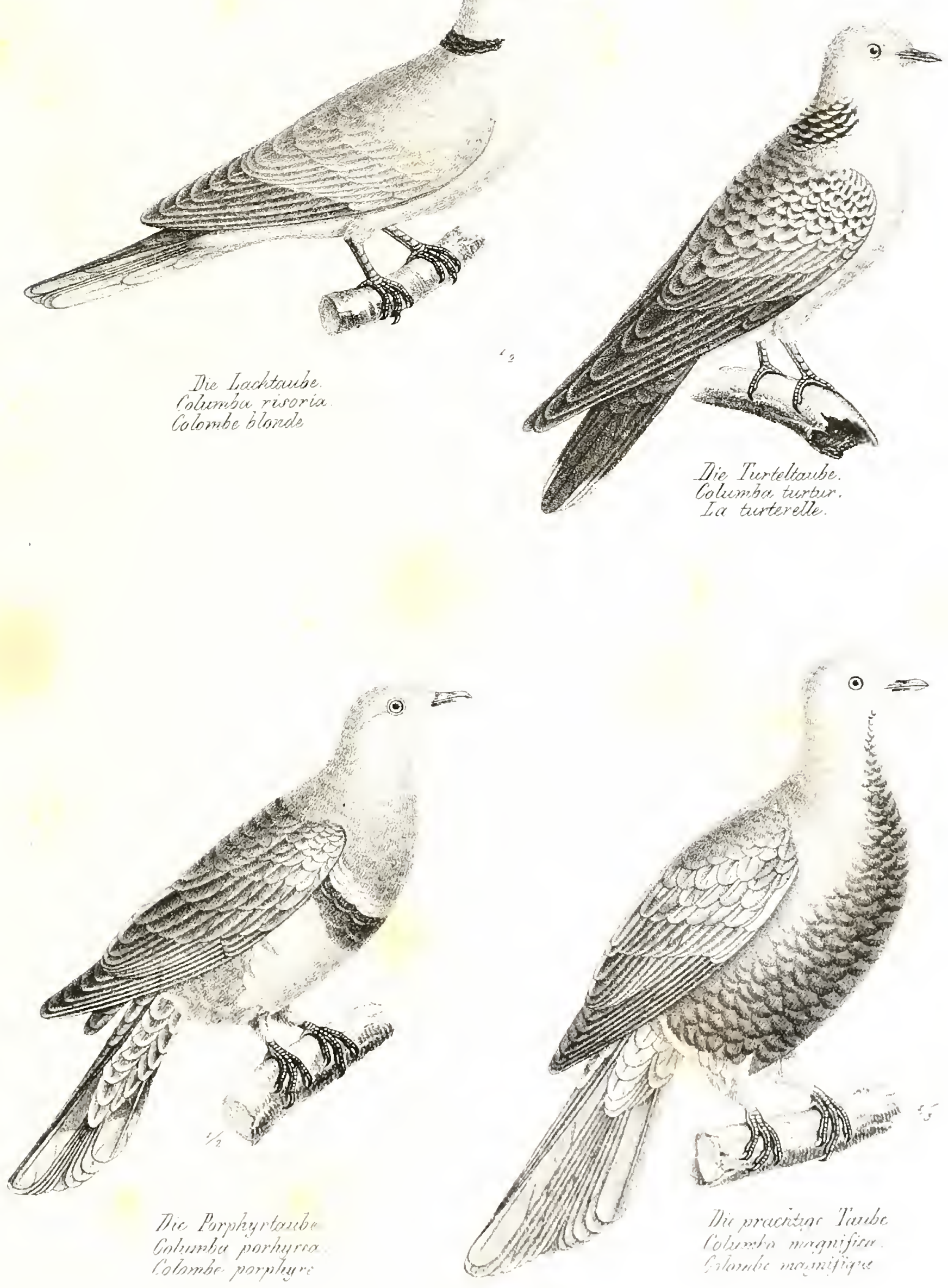


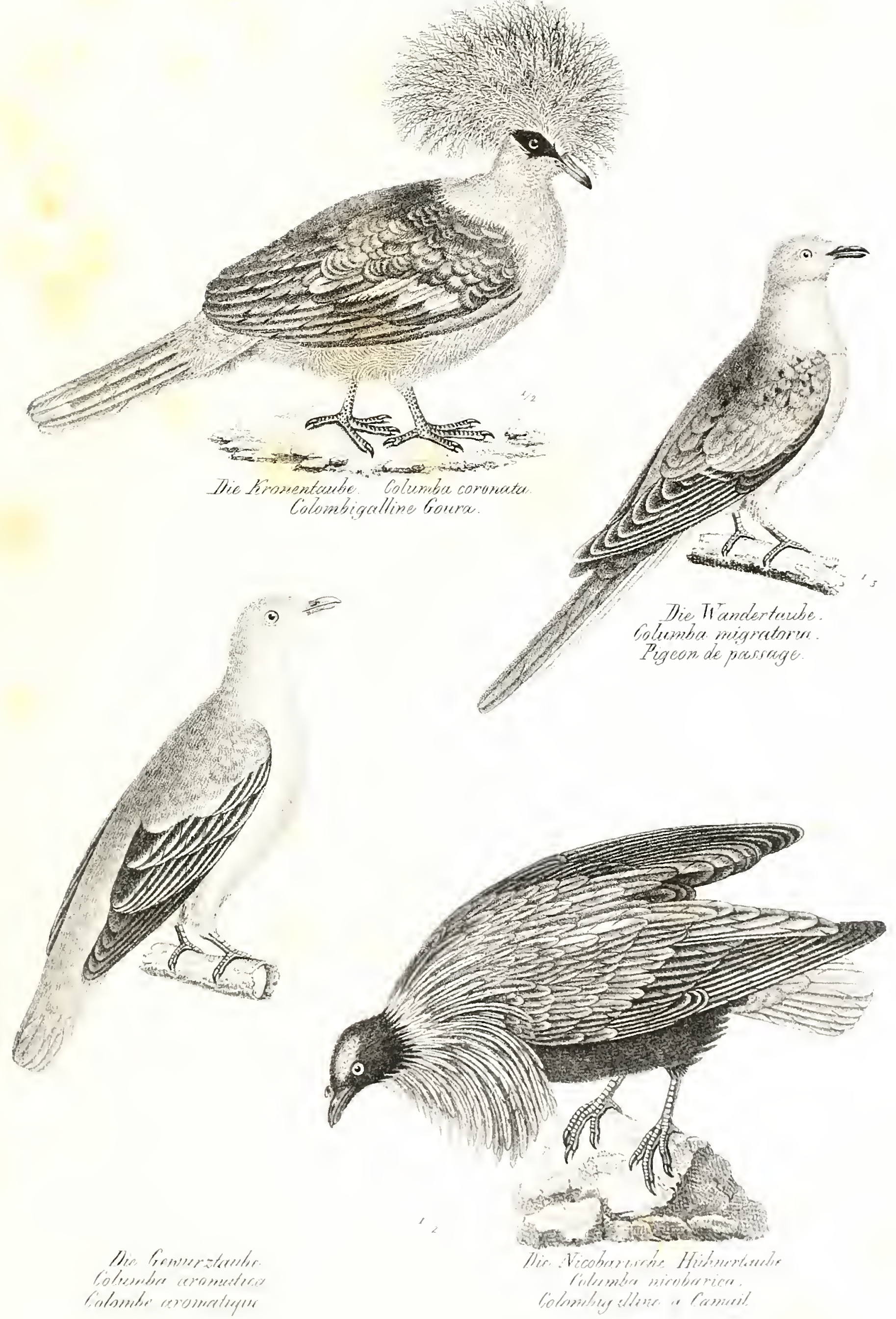





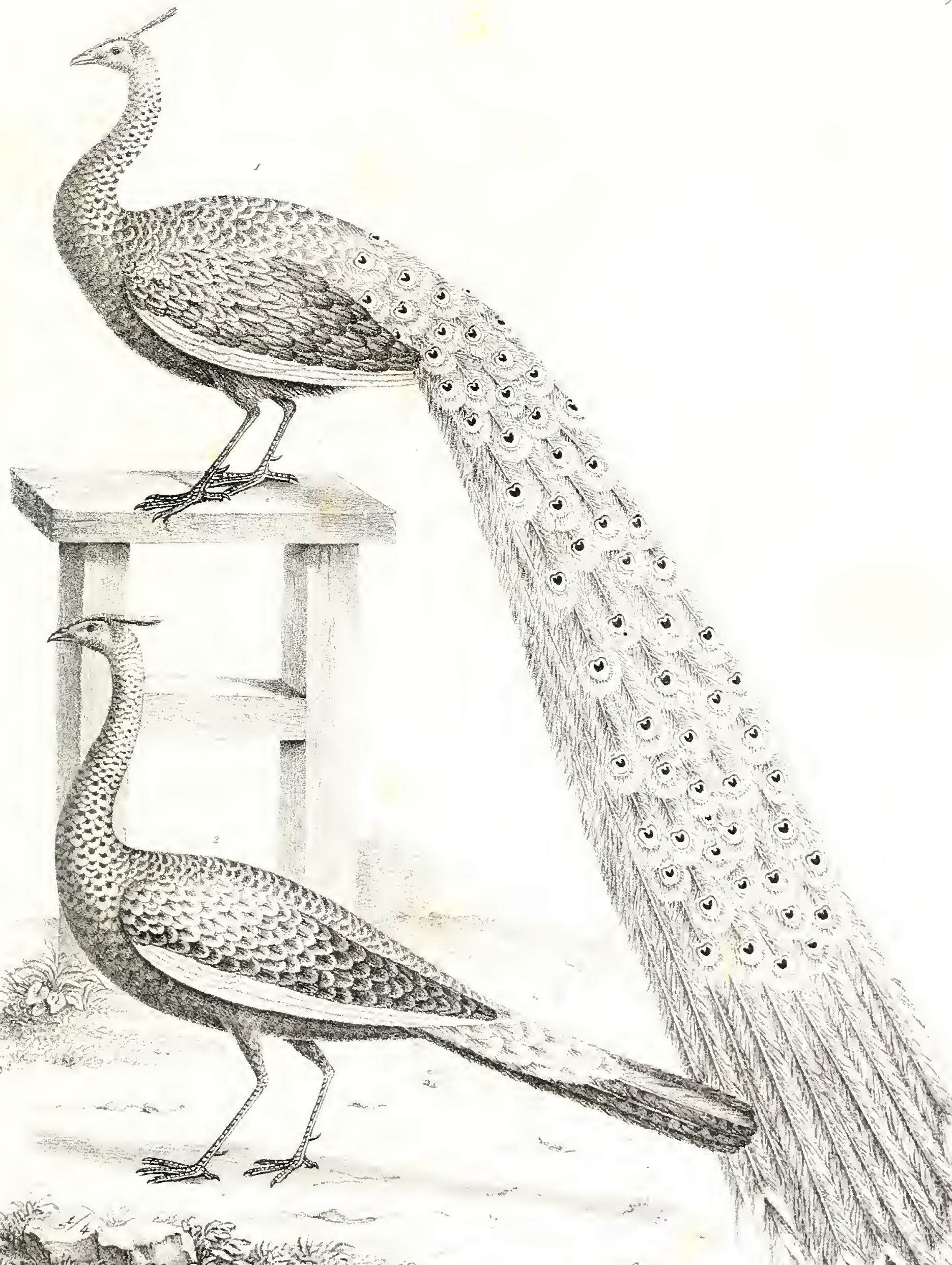

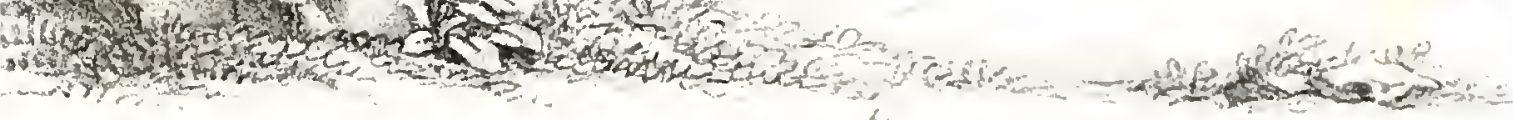

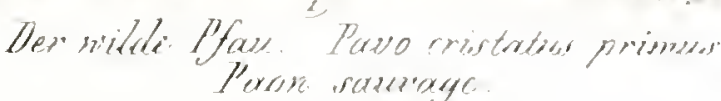




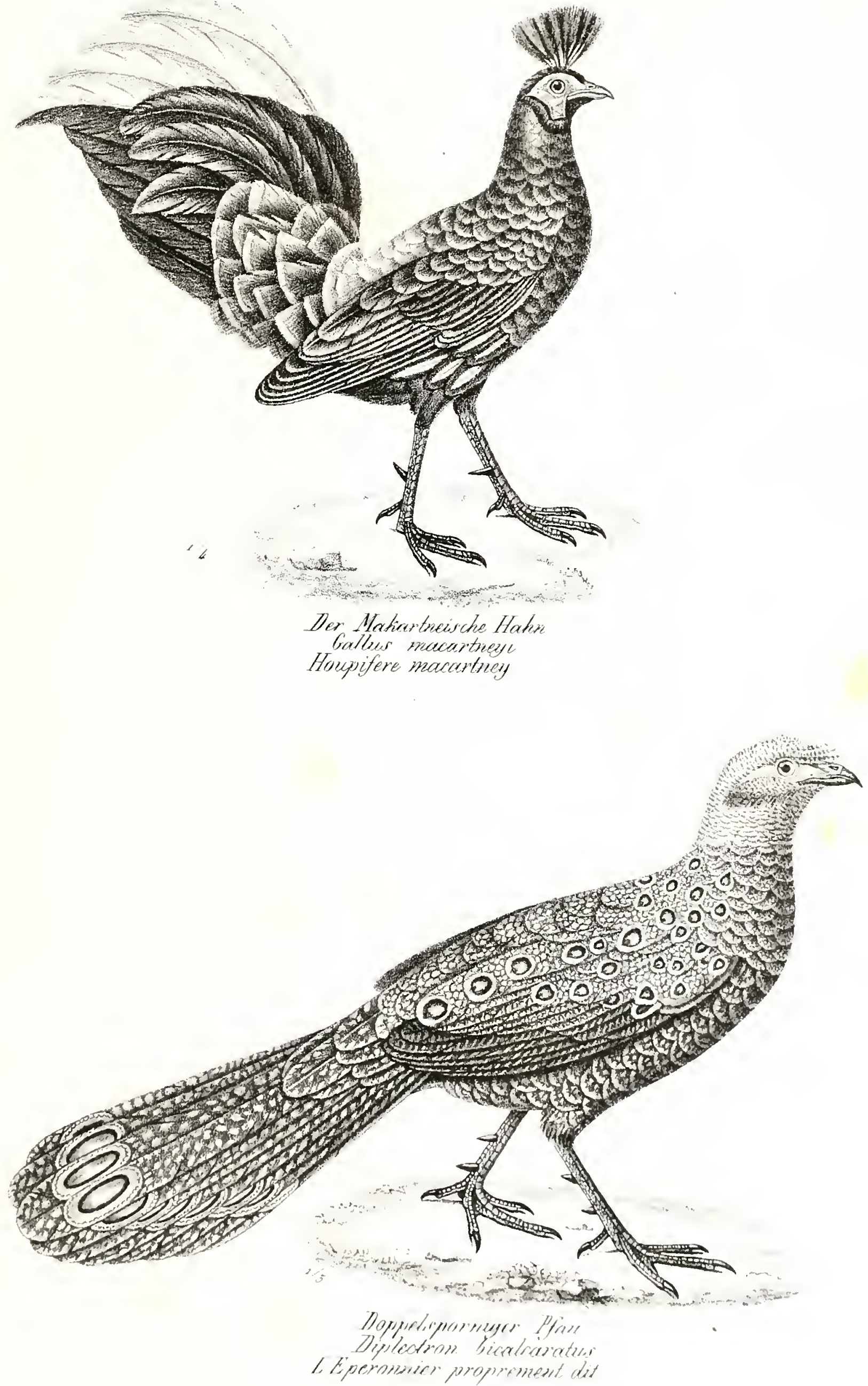



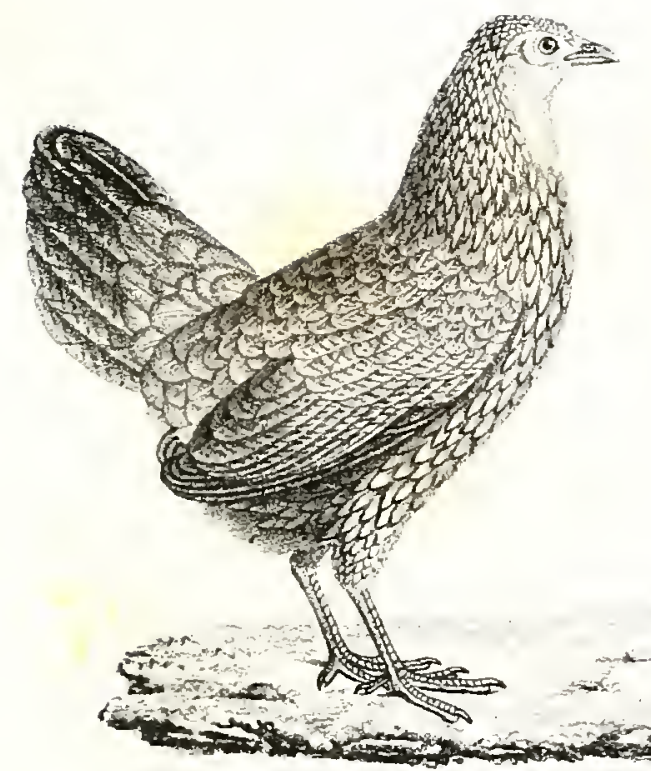

Sonnurats Herne.

Pule Sornerut

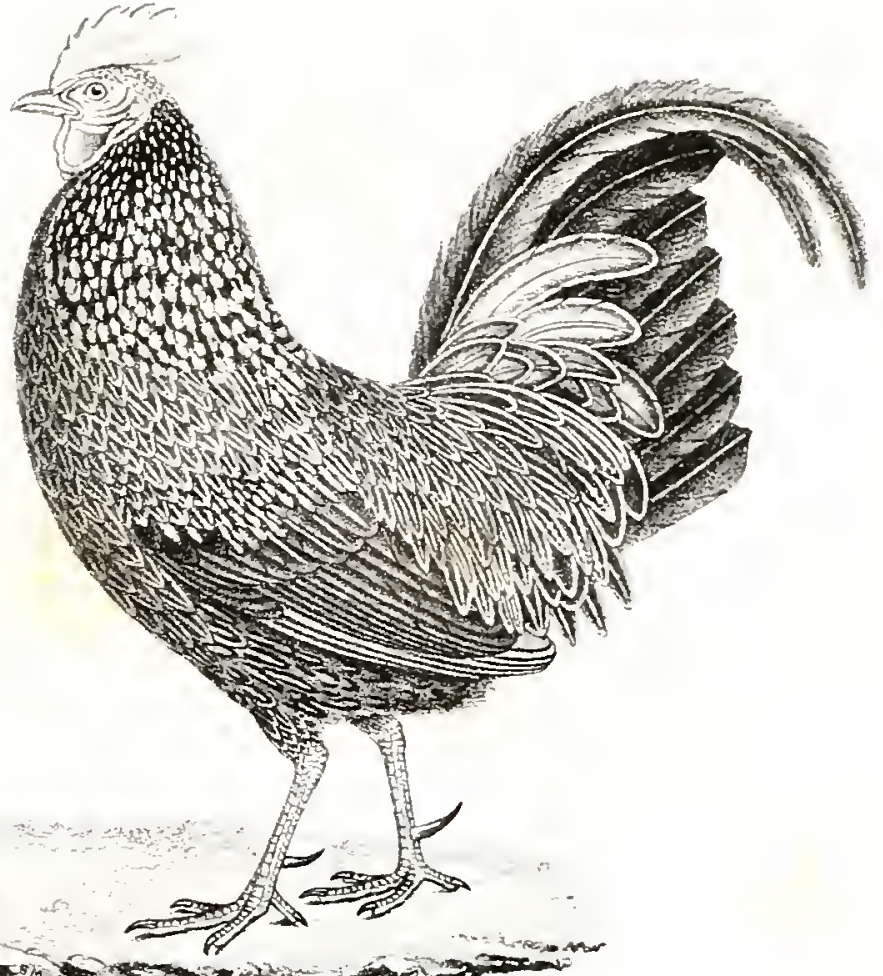

Gonnerats Itahn

Gallens Sormercats.

Coy Somierat.

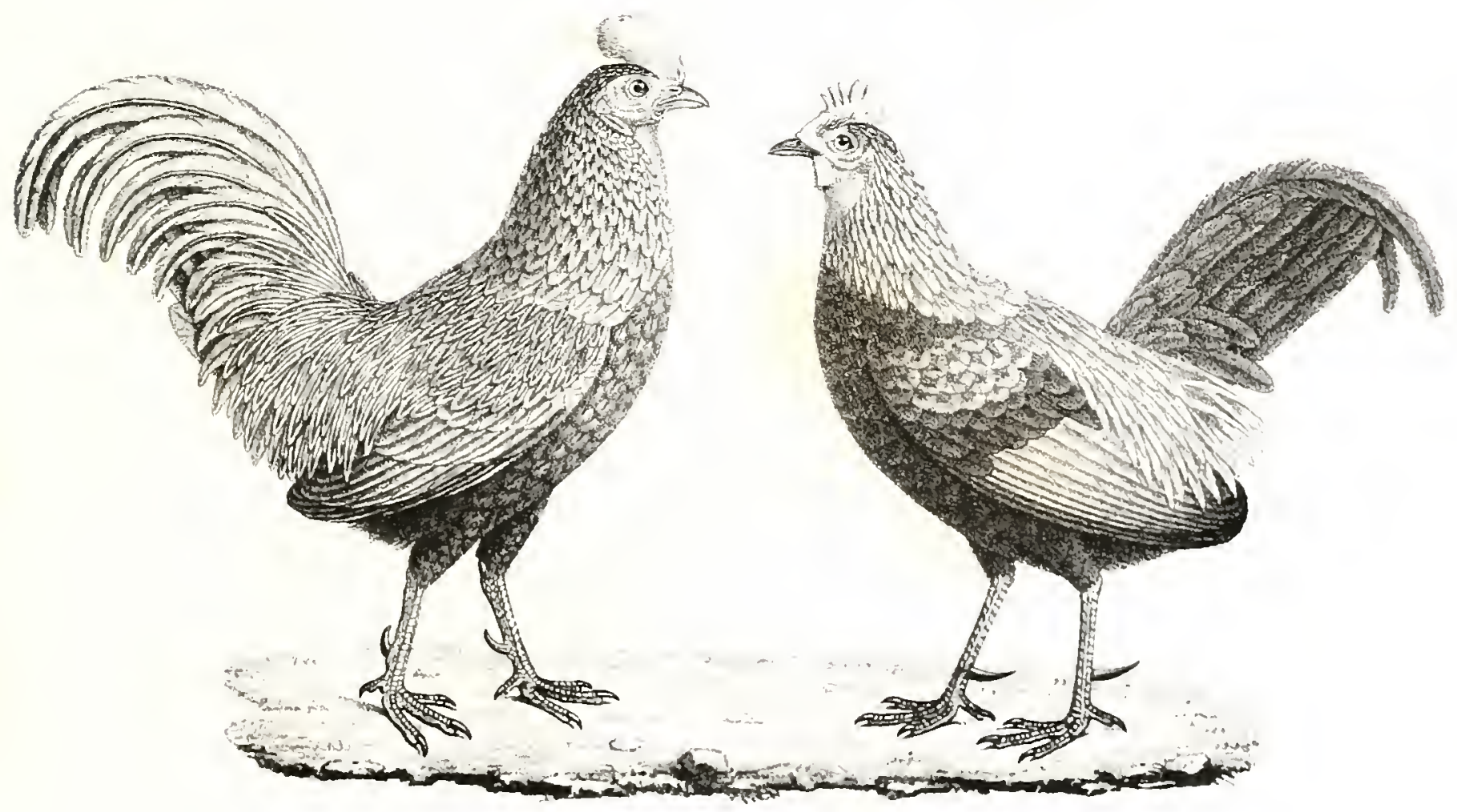

Der hupferfarbme Haho Gallew aeneevs. Coy bronzé

Hahne vors Bantivers Gallise Birentiven liog biantivine 


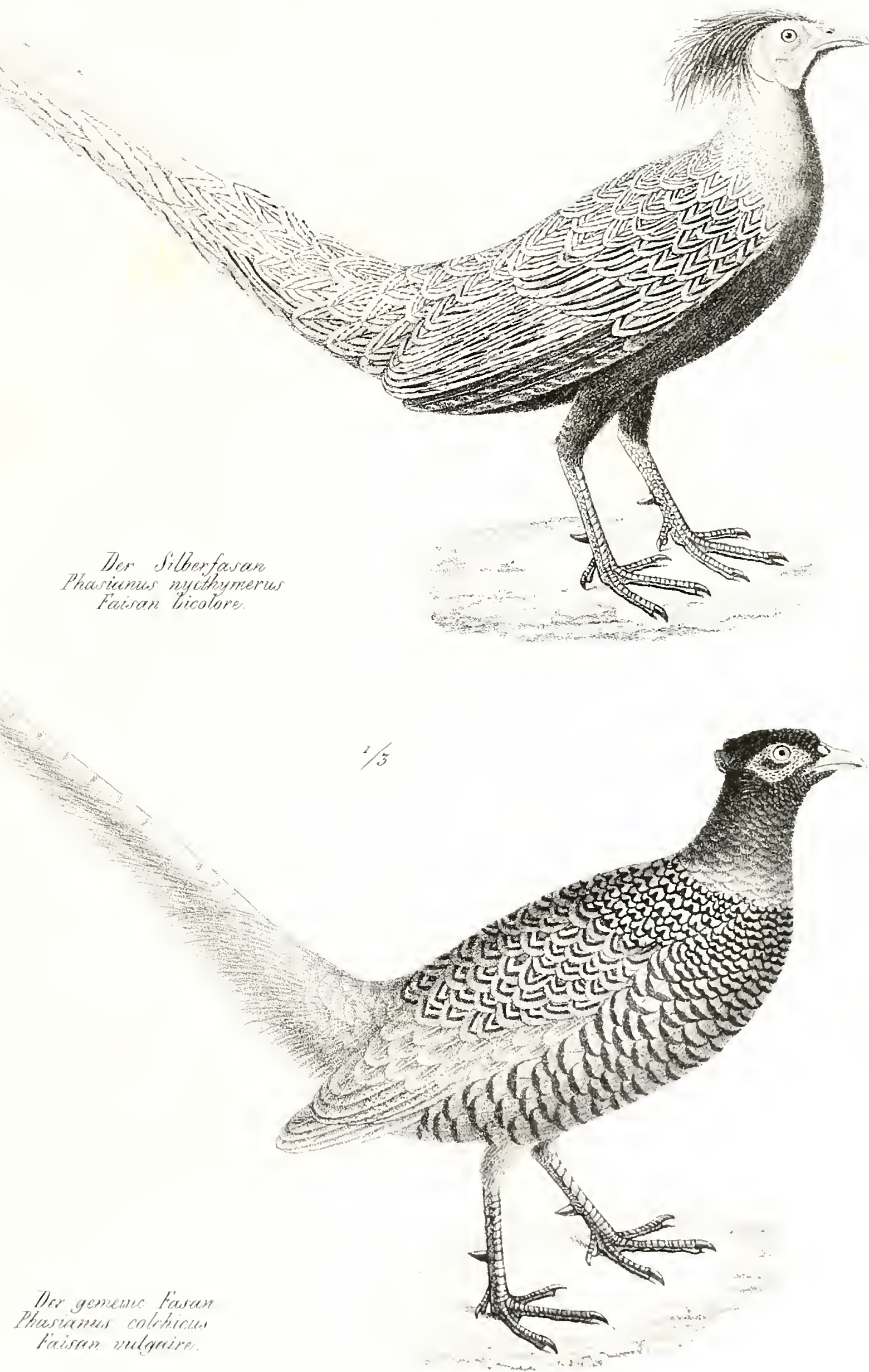



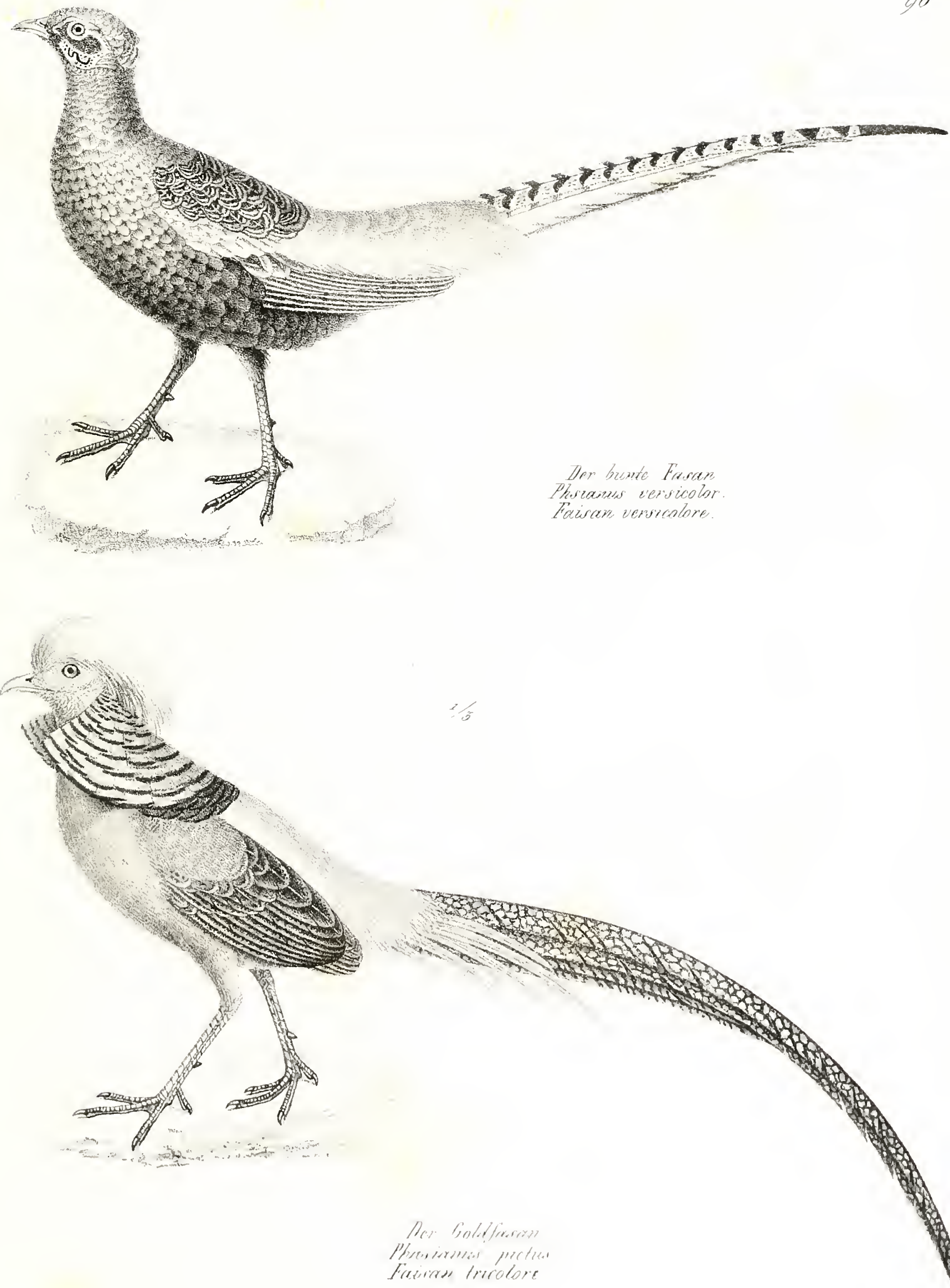





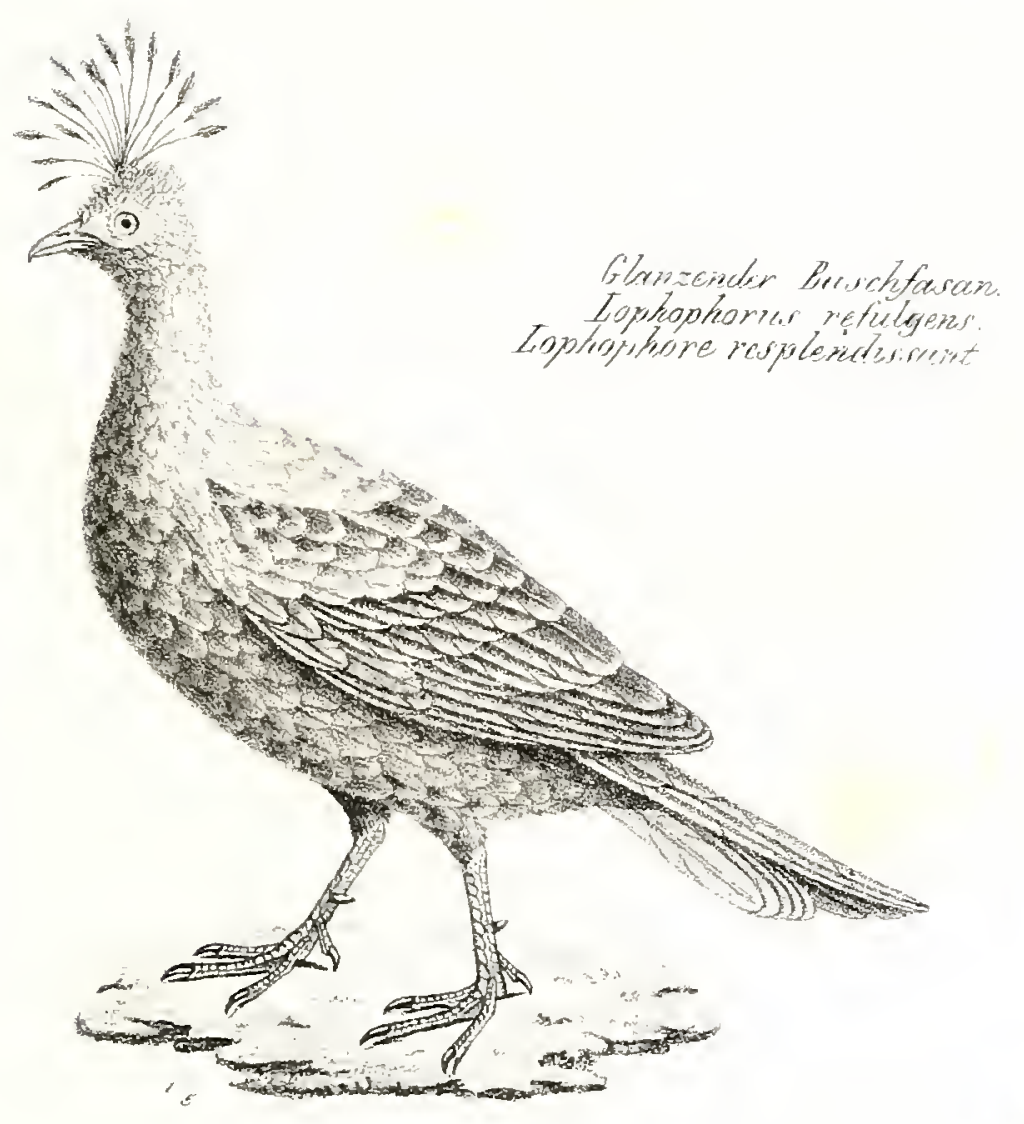





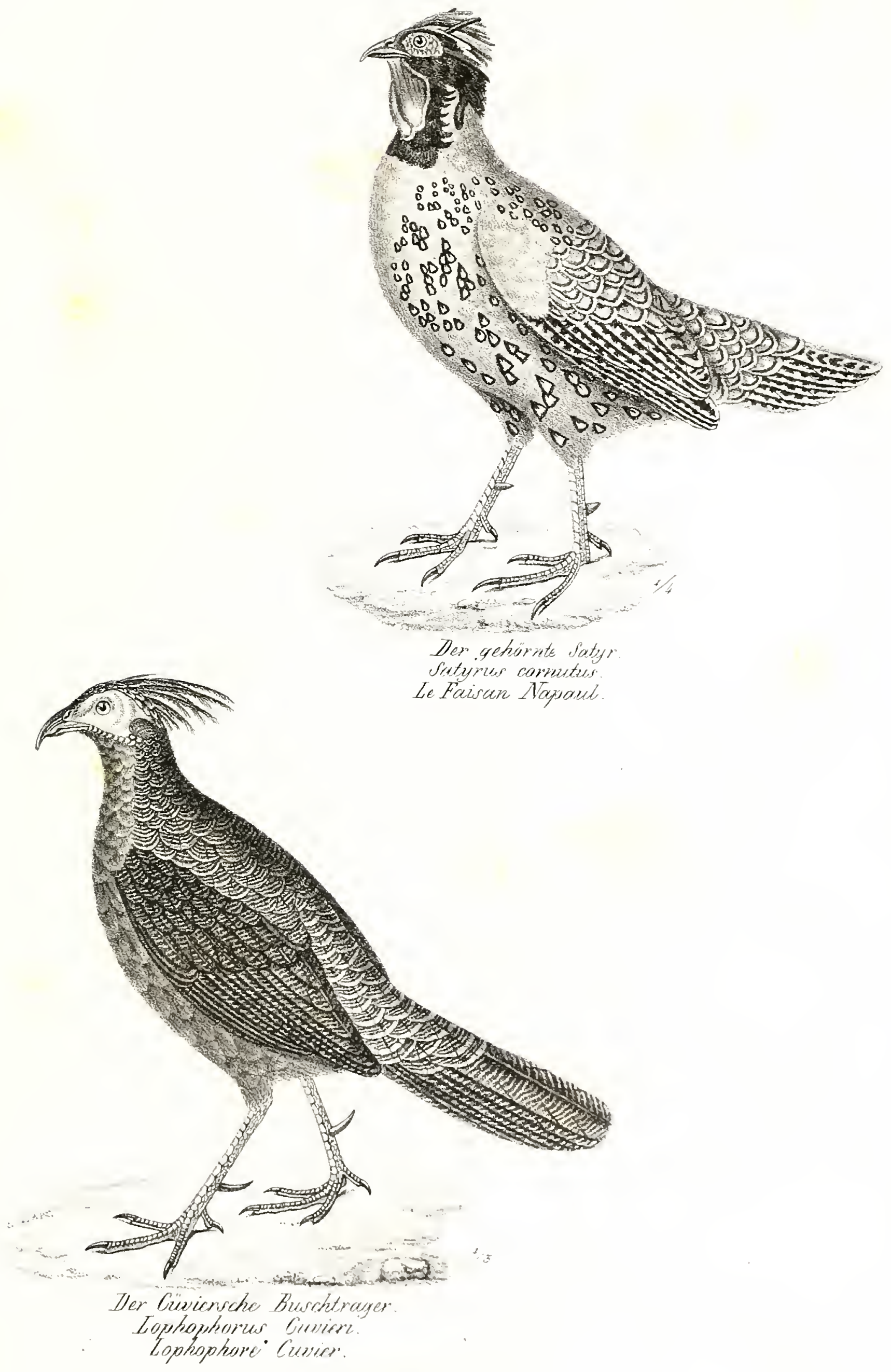




\section{、}

$\infty$ 


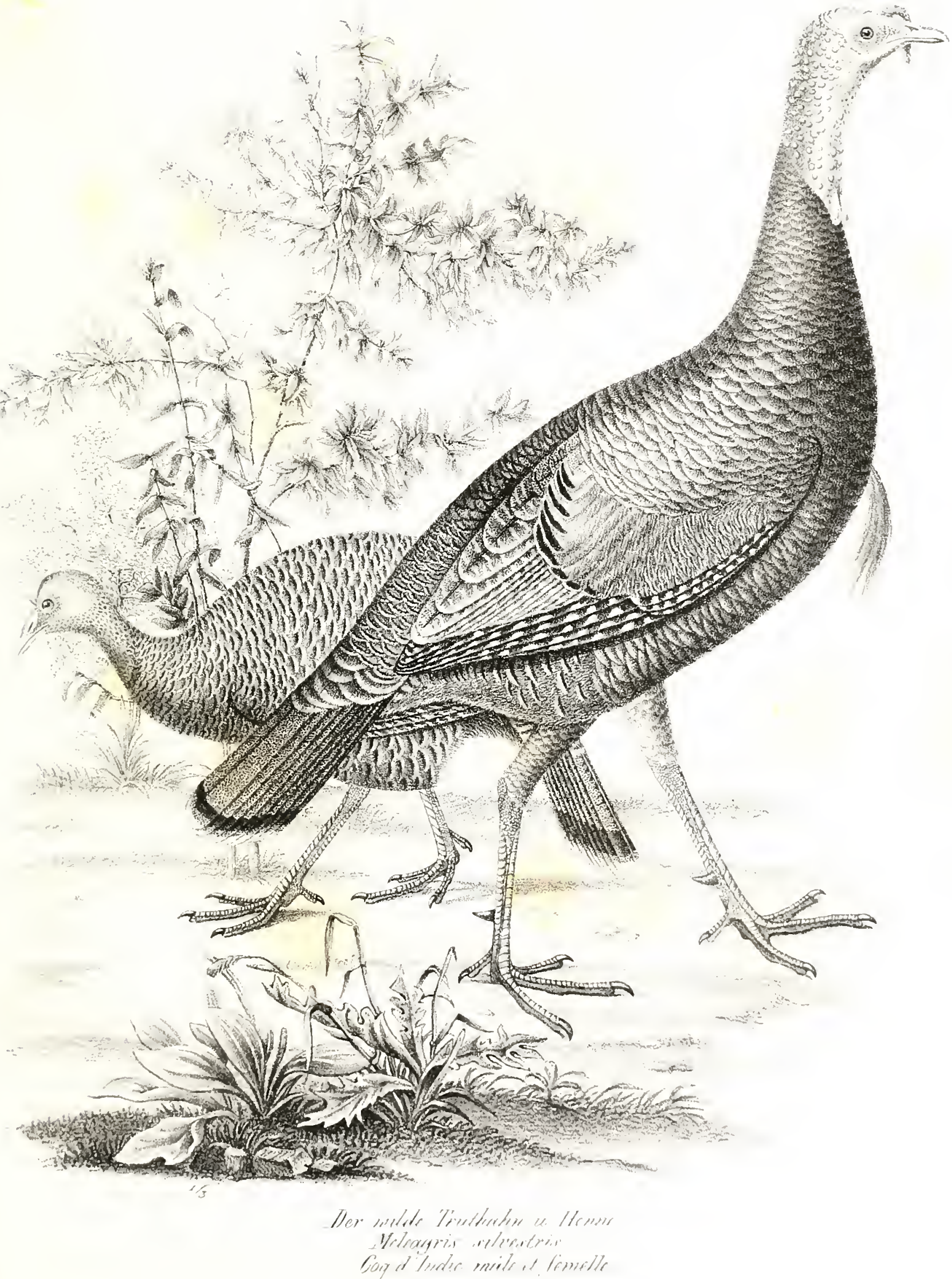




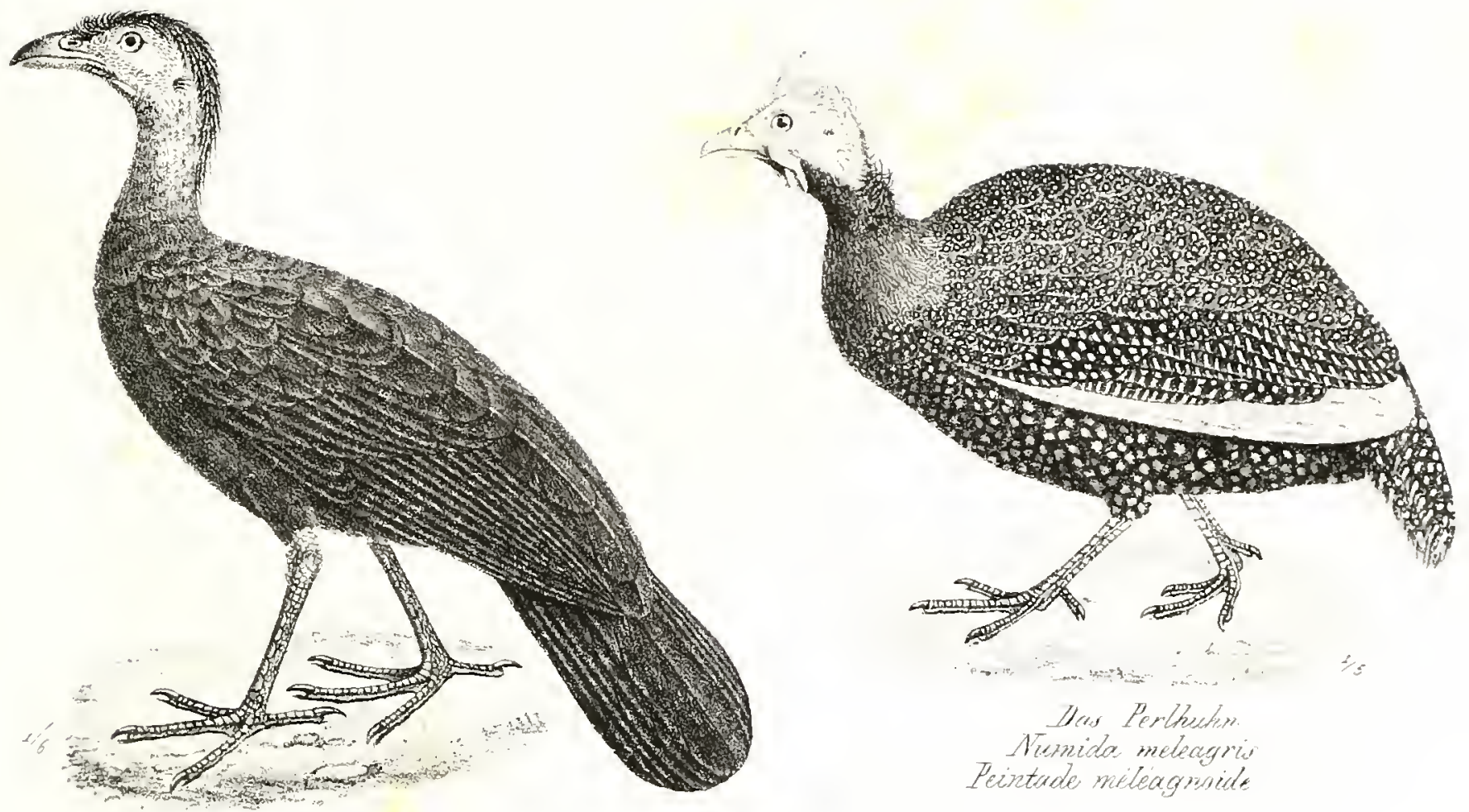

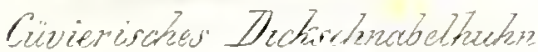

Tategathe Cunier

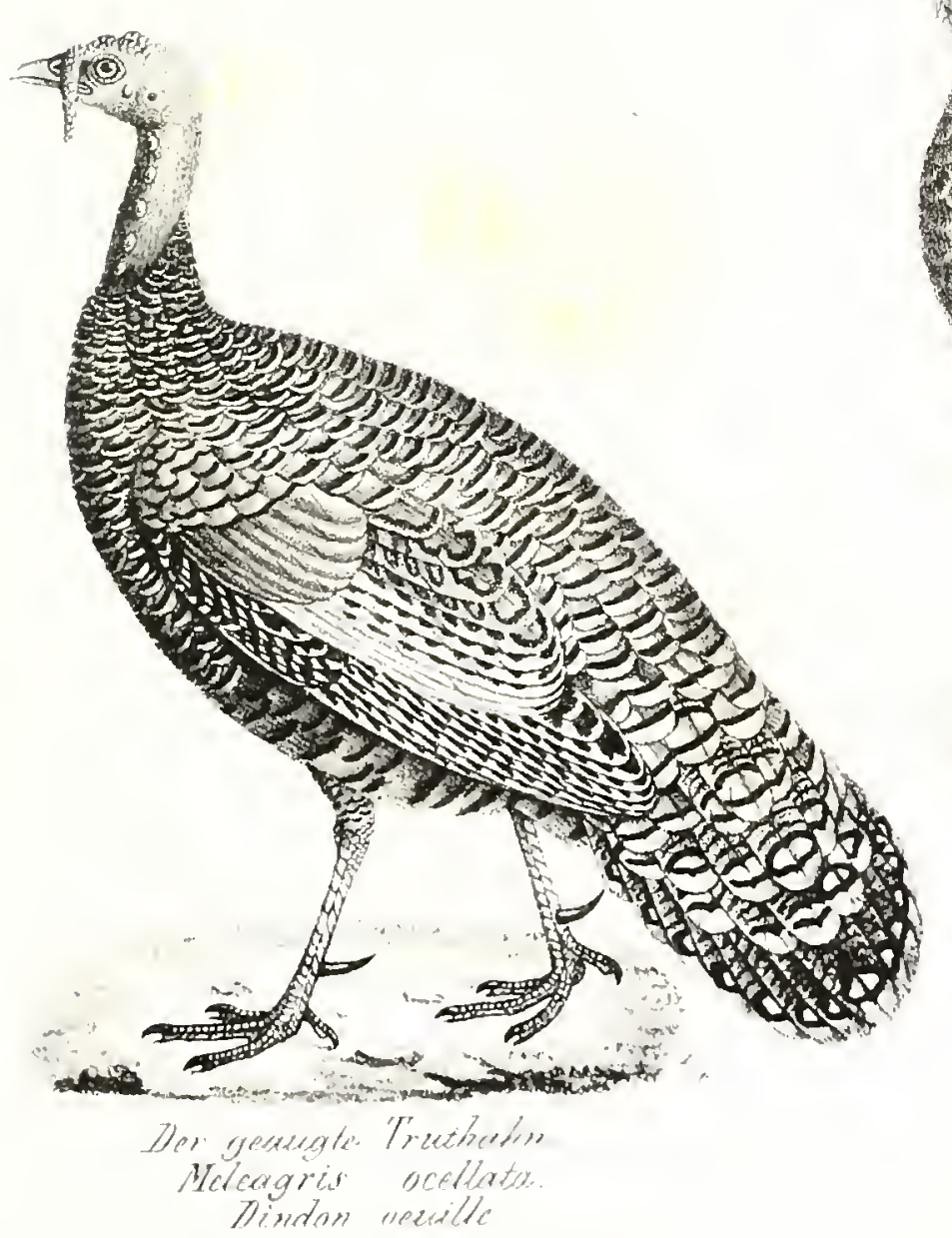

(2)
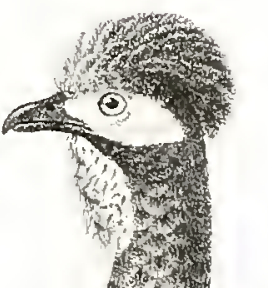

1)

$+67$

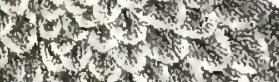

H.

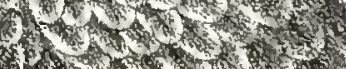
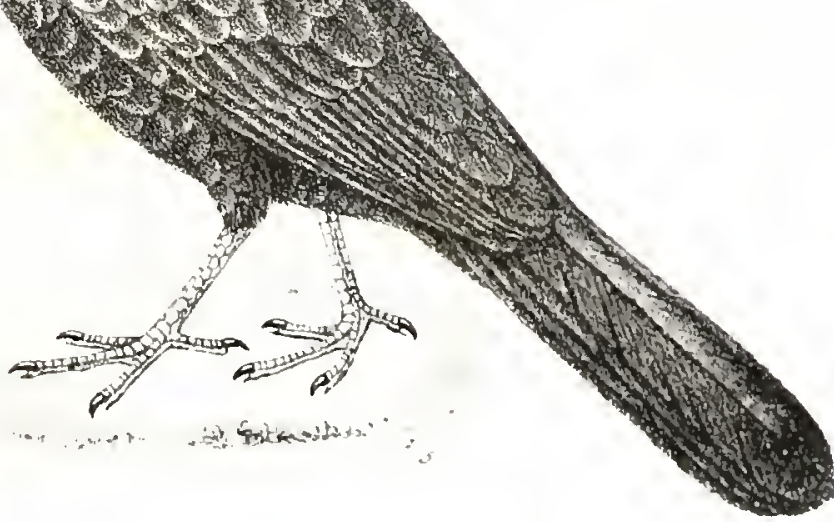

Den Herowel.

Tla nelope Irand?

let Nerwil 



$$
\text { h }
$$




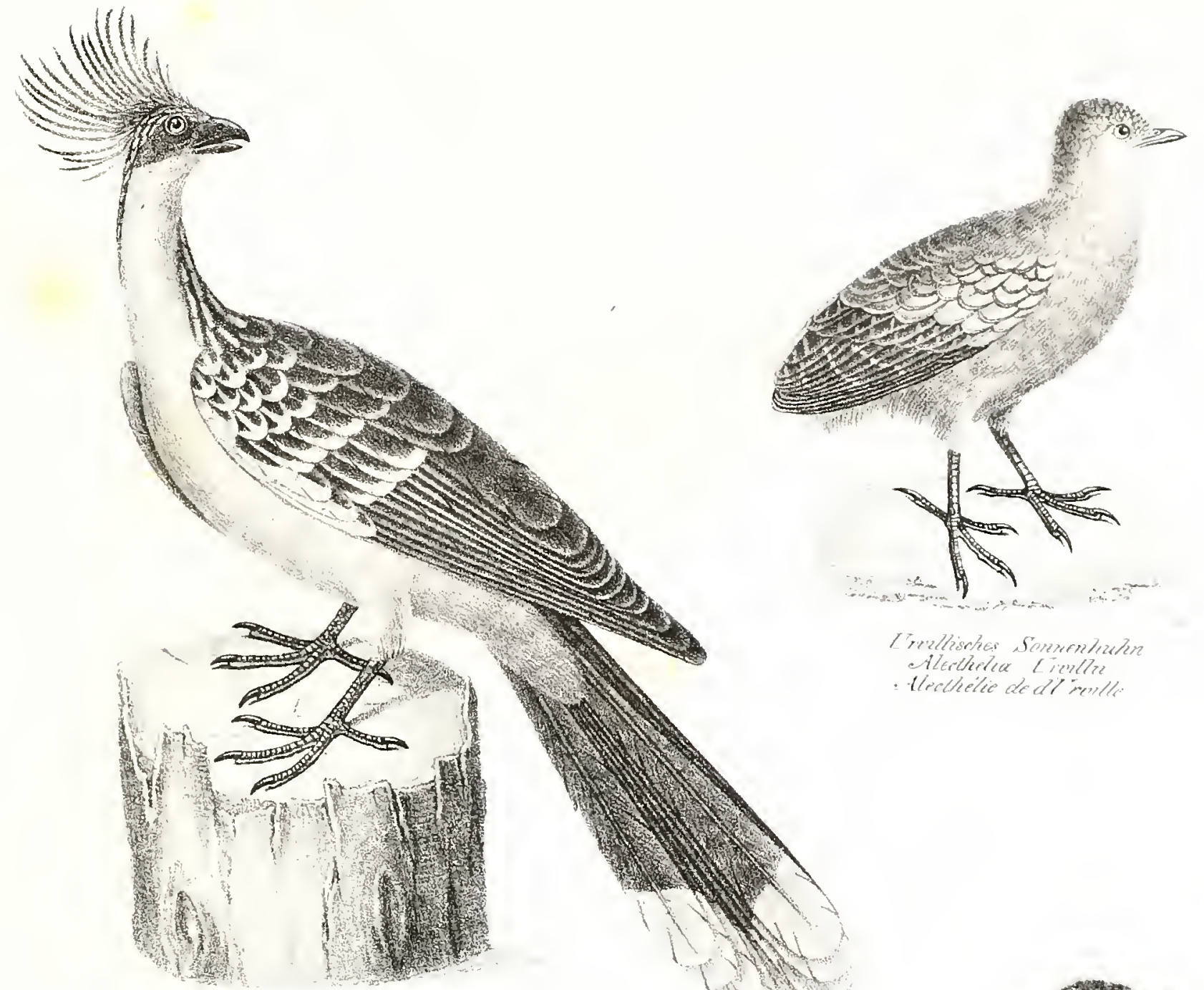

(1)?

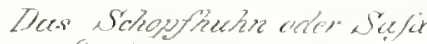
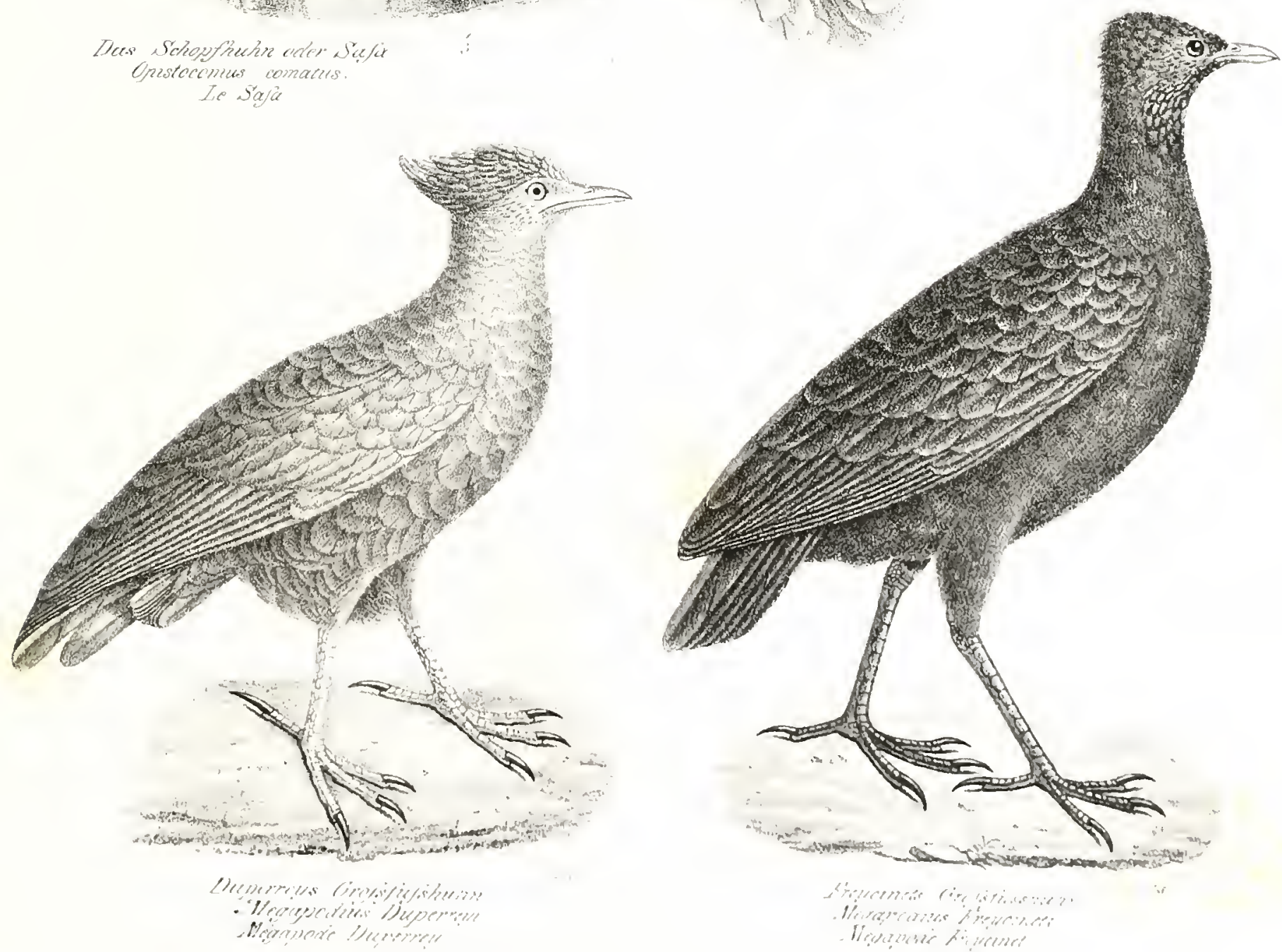


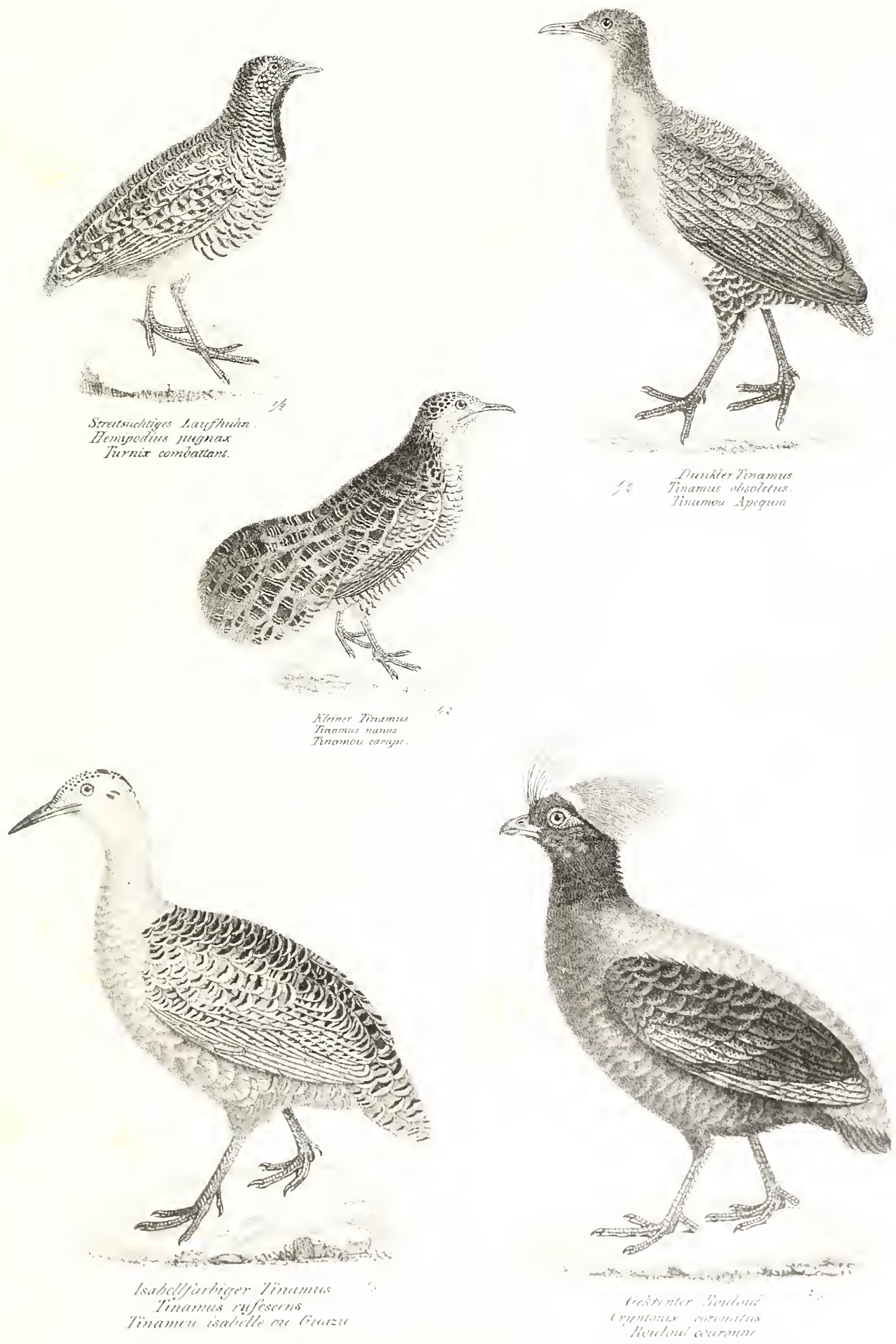



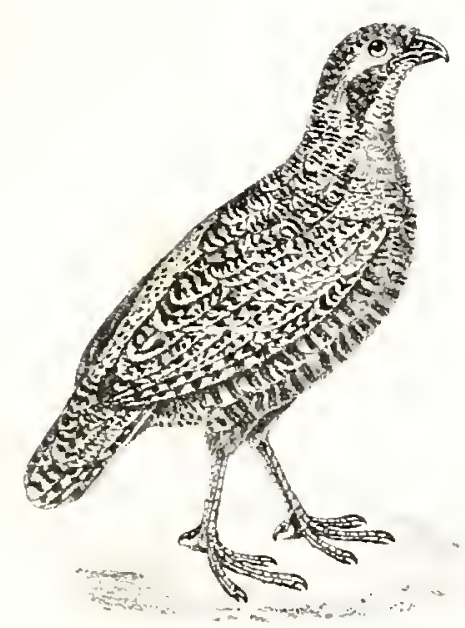

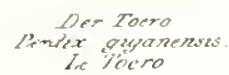

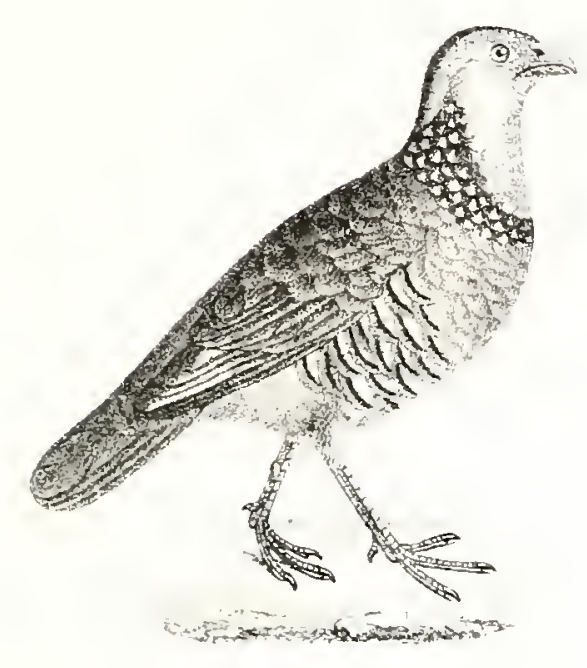

Das Yippenketh Perdir pezrond
Perdiser greselens

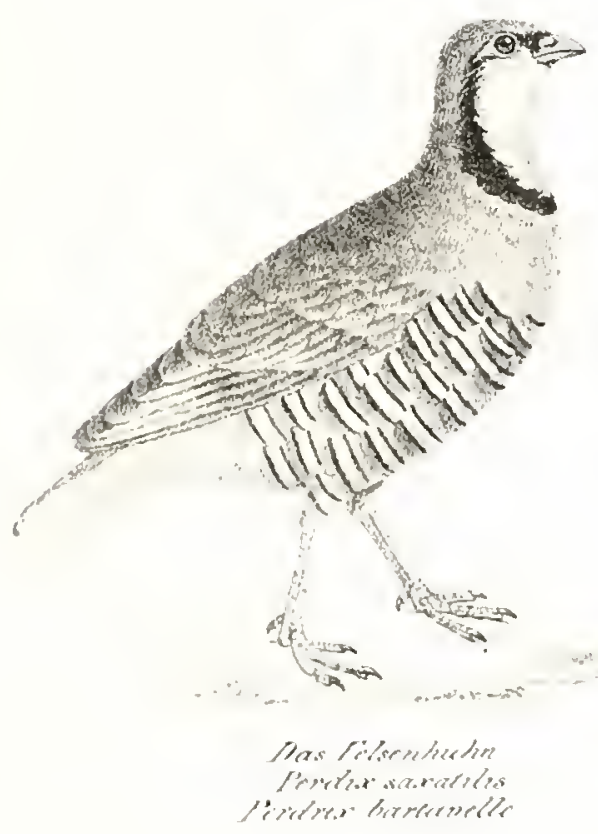

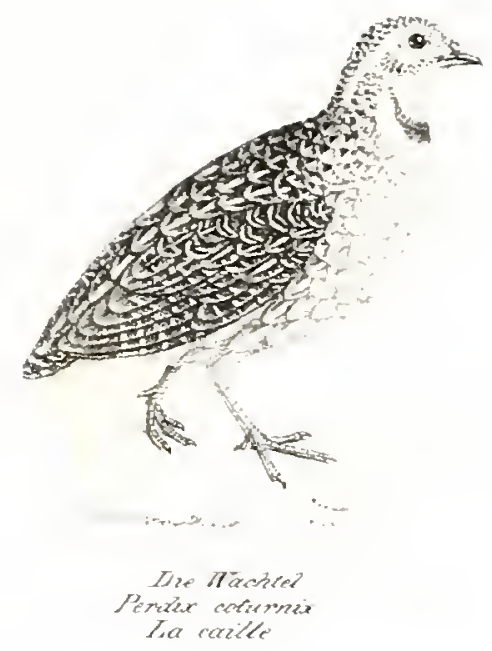
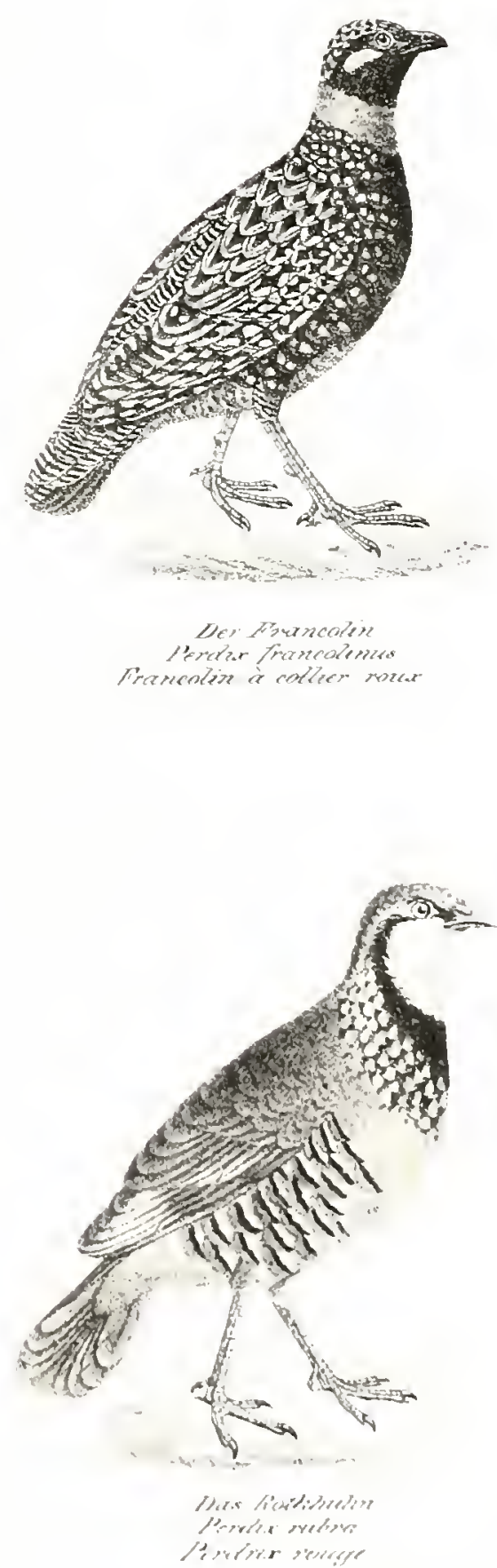


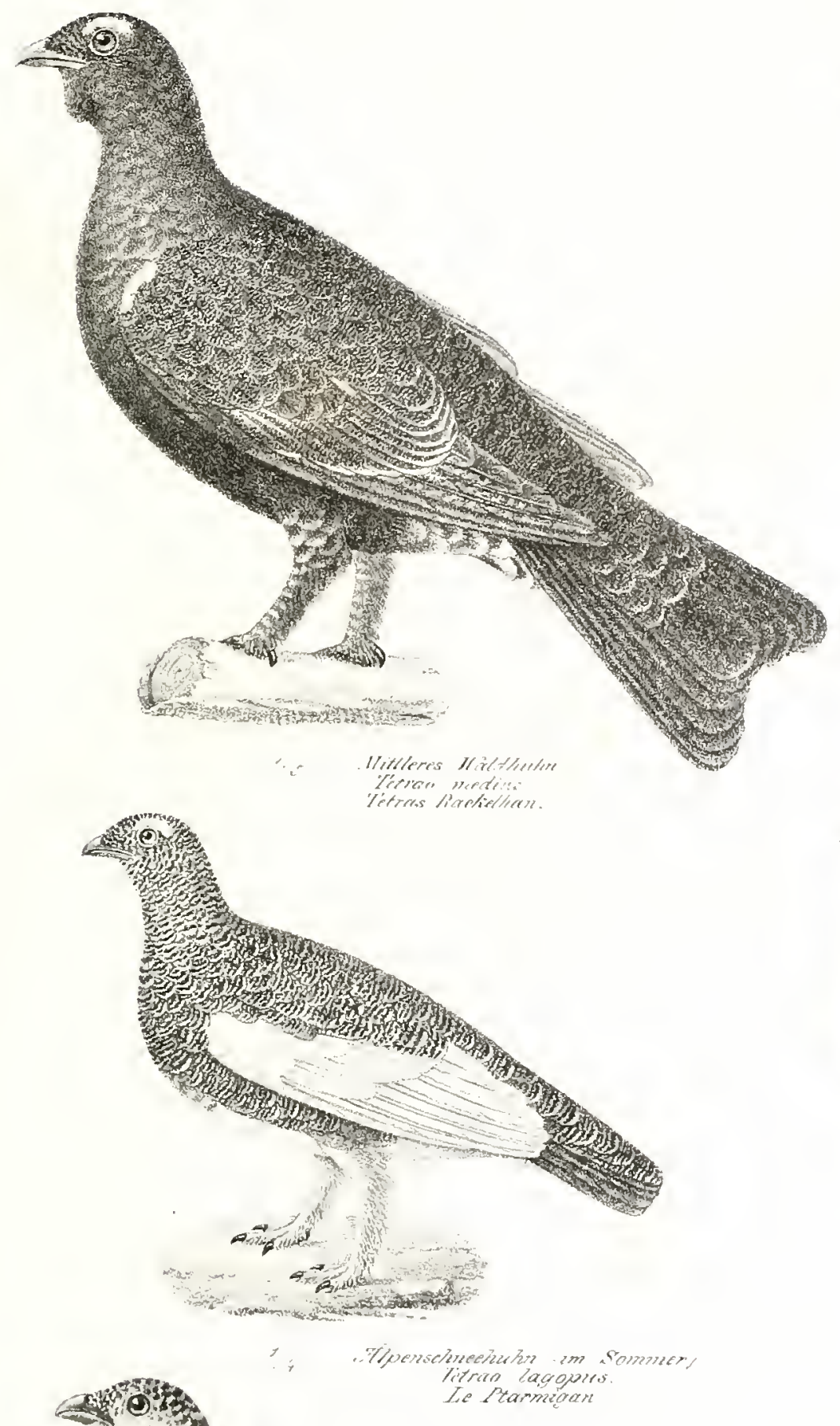

$$
\text { is }
$$

11.).
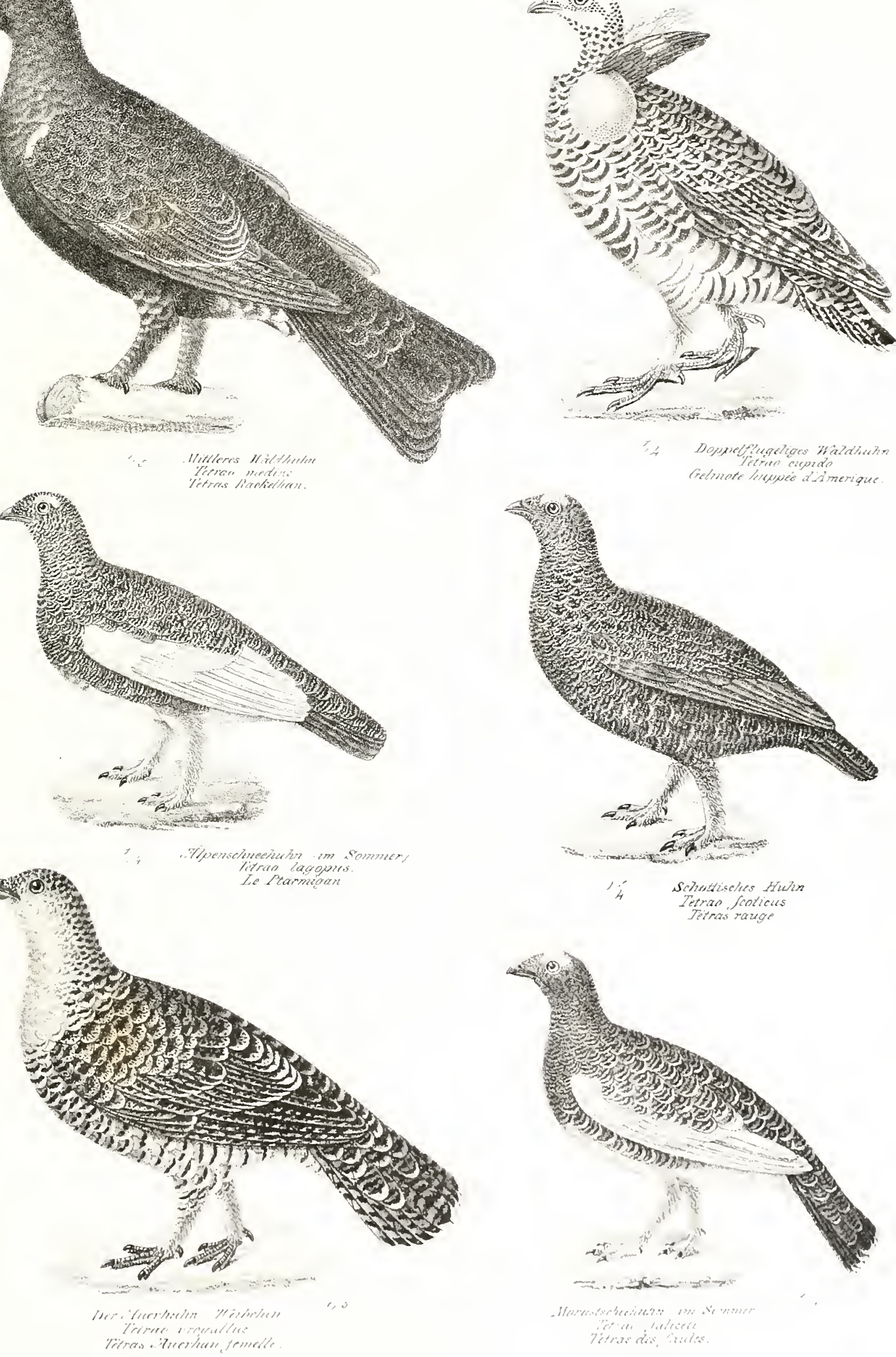


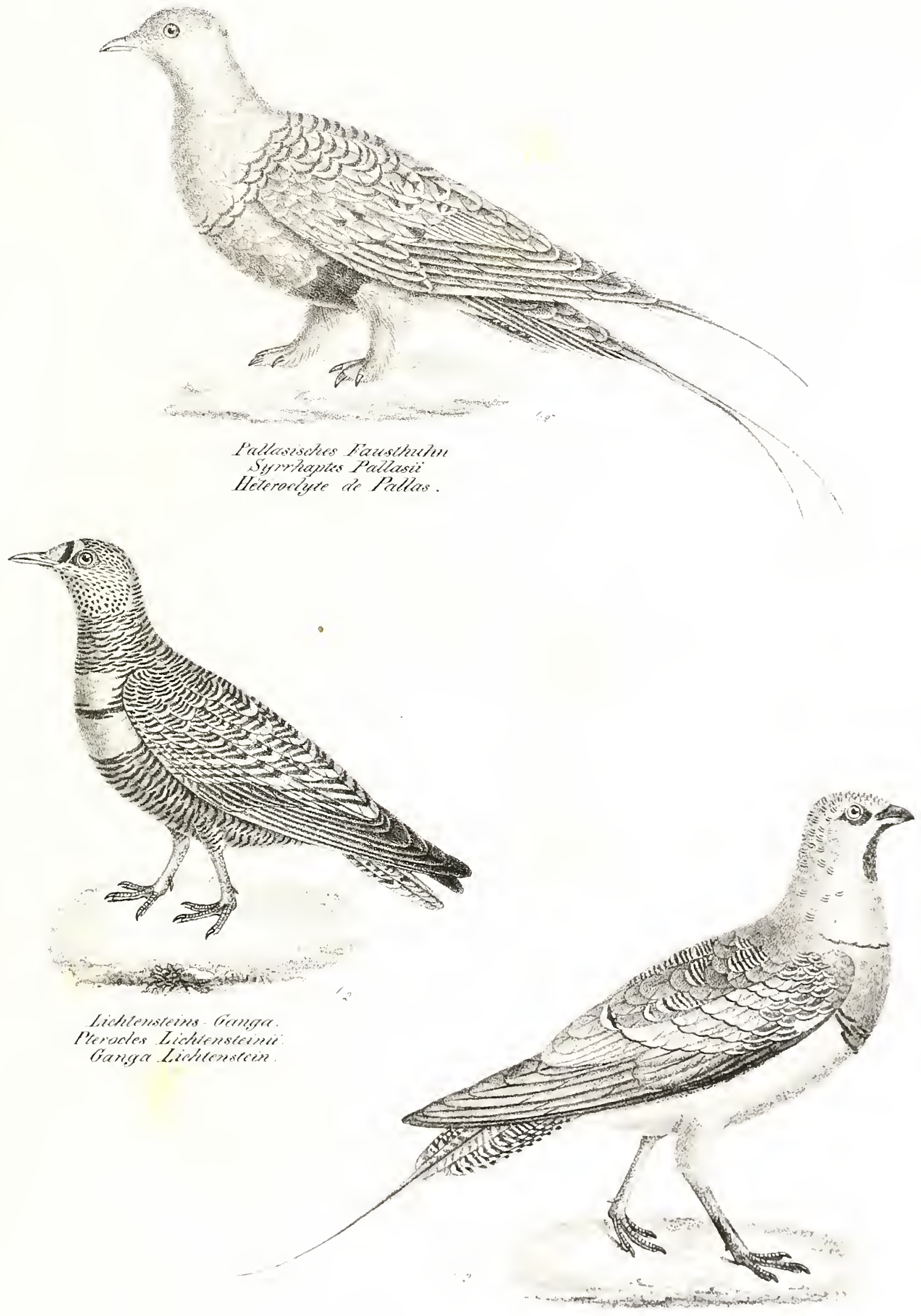

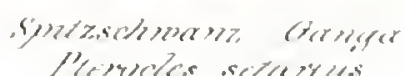

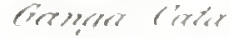





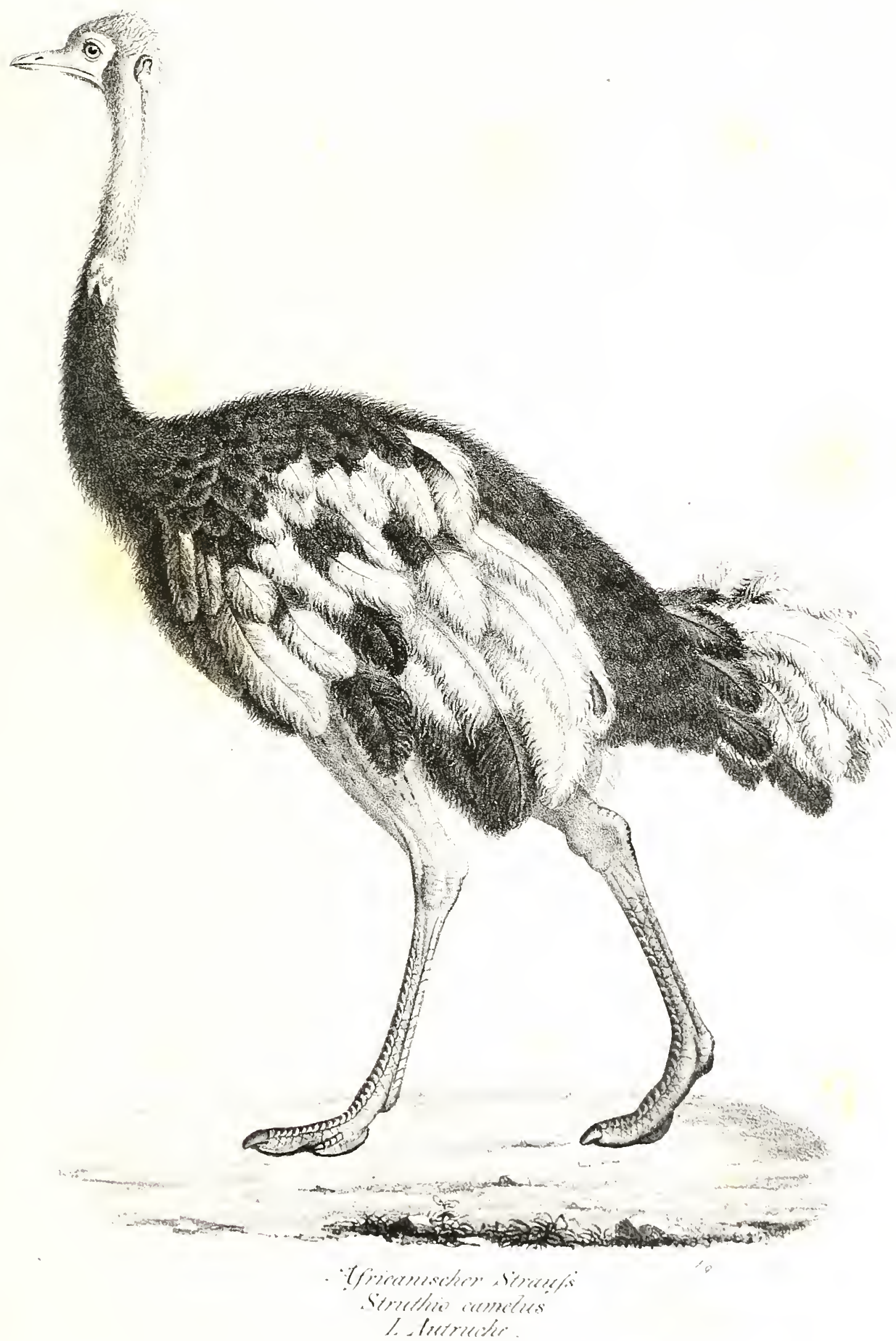





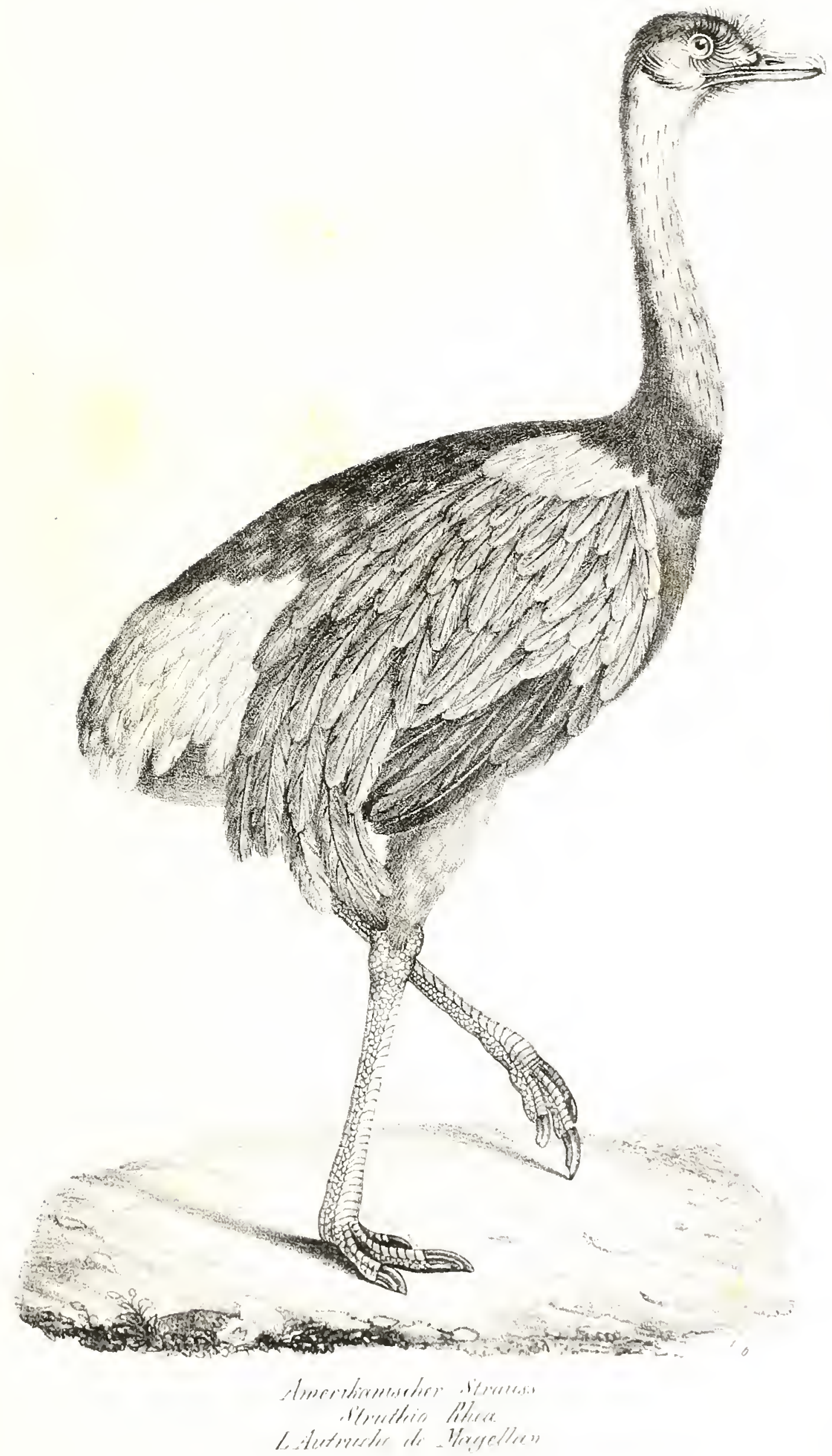




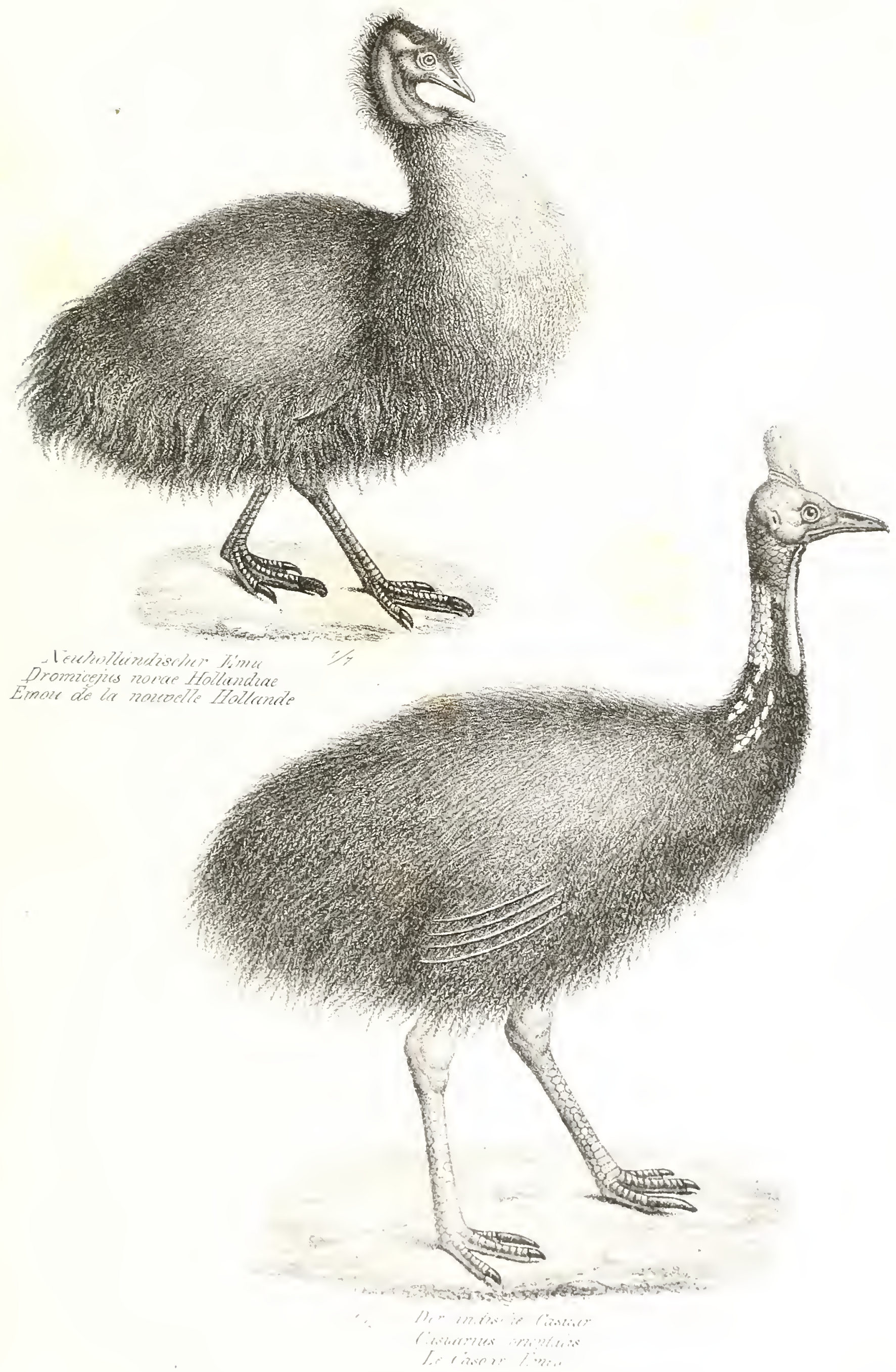




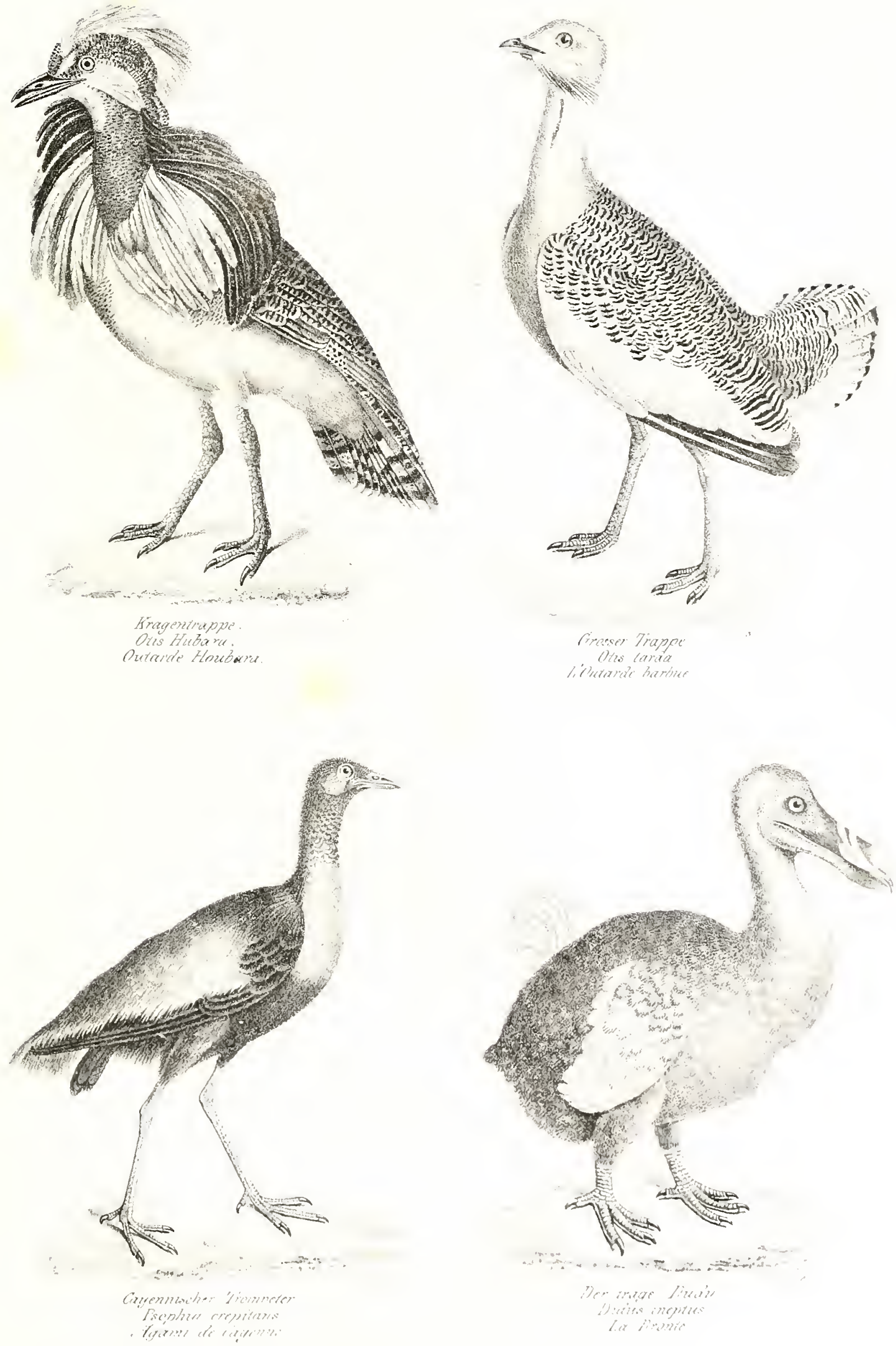

$-1$

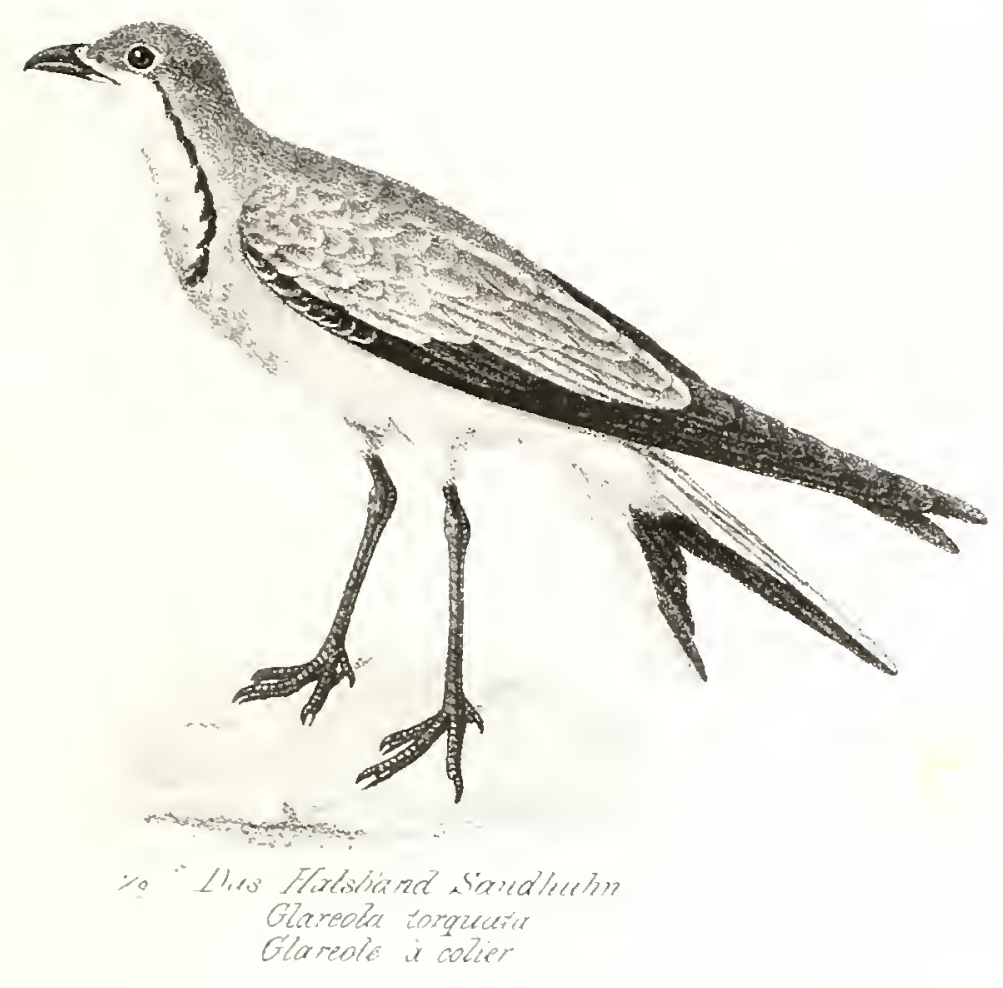

WW
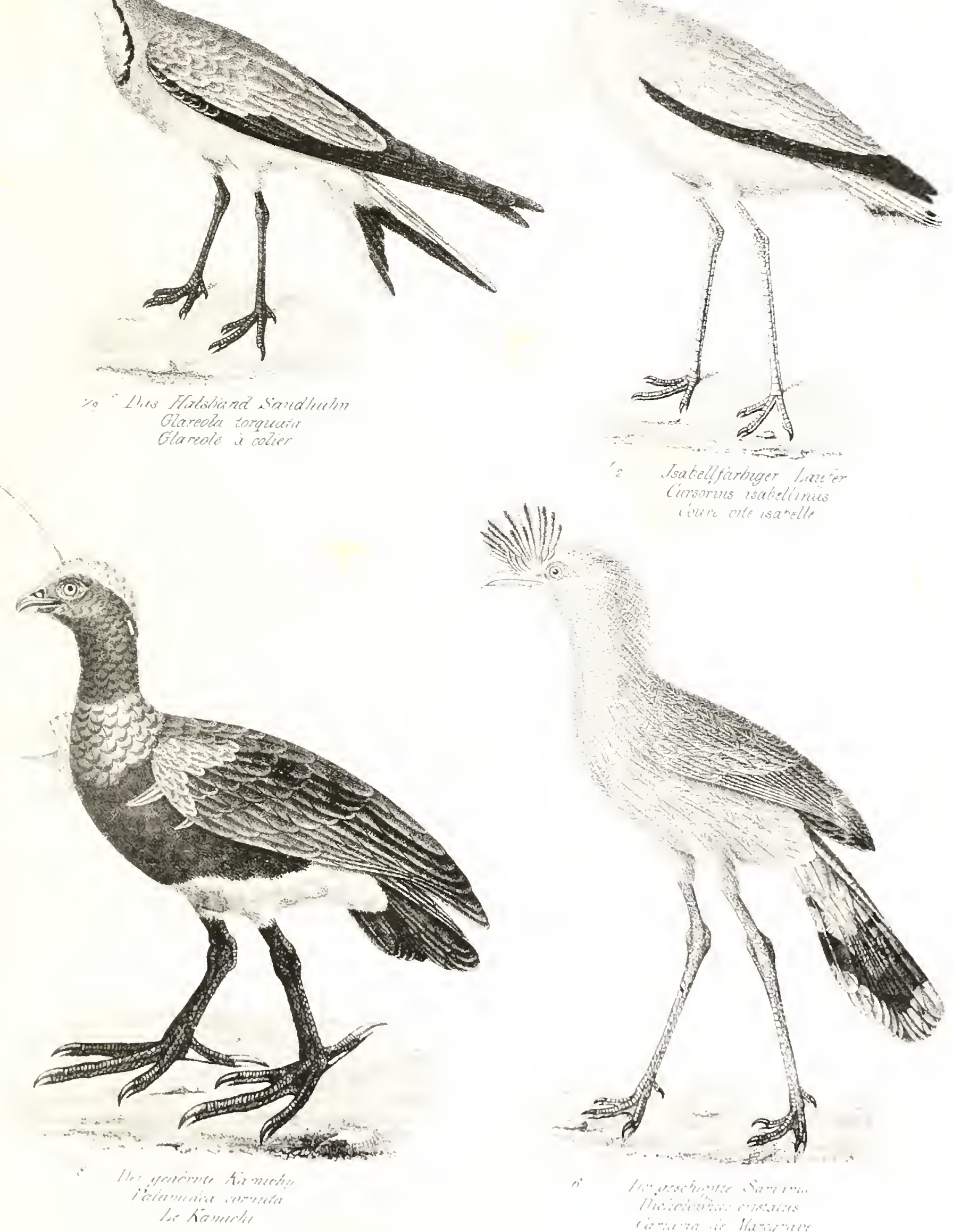

wituc inte usabelle

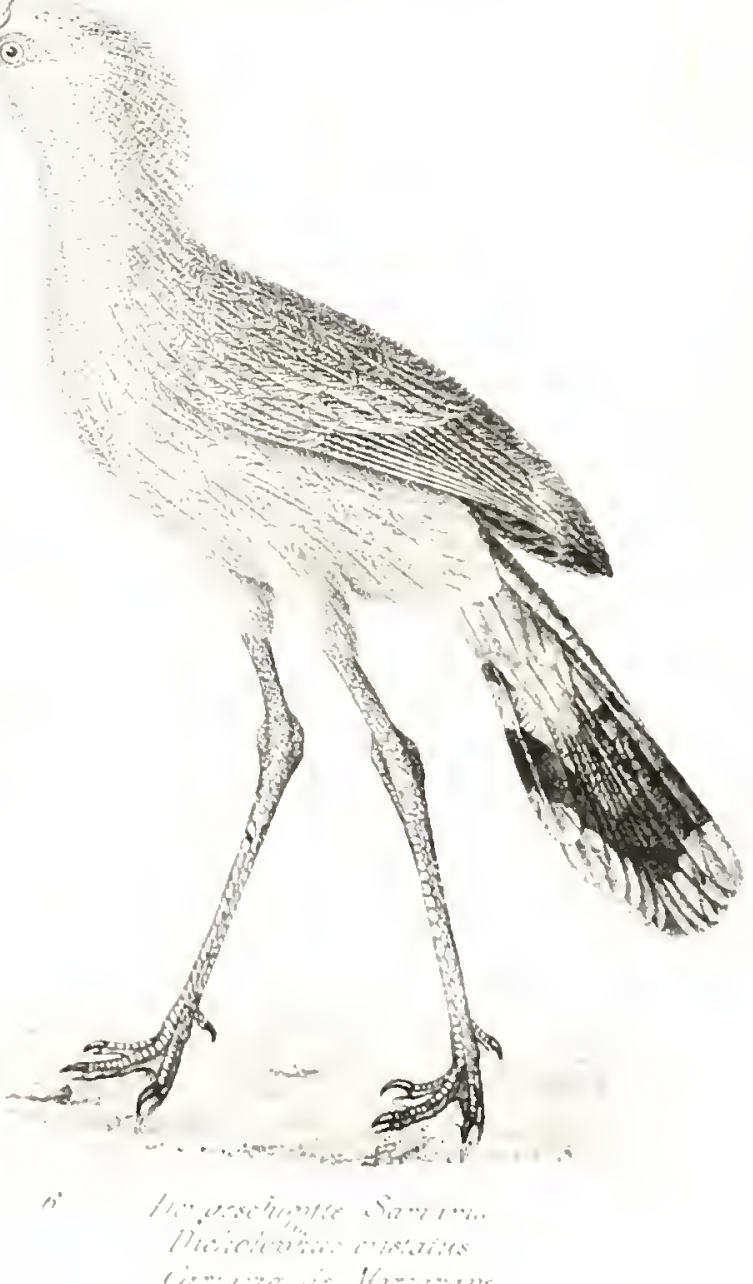





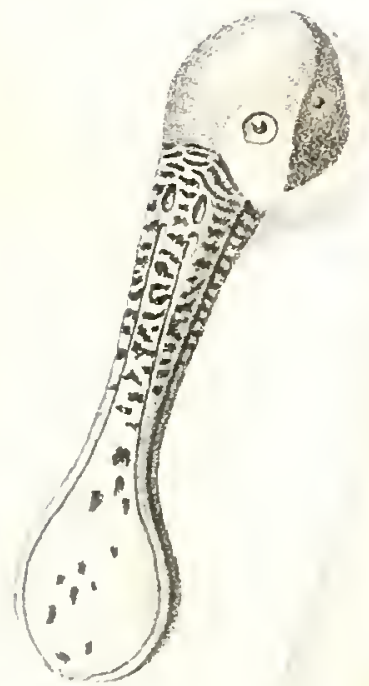

(e)
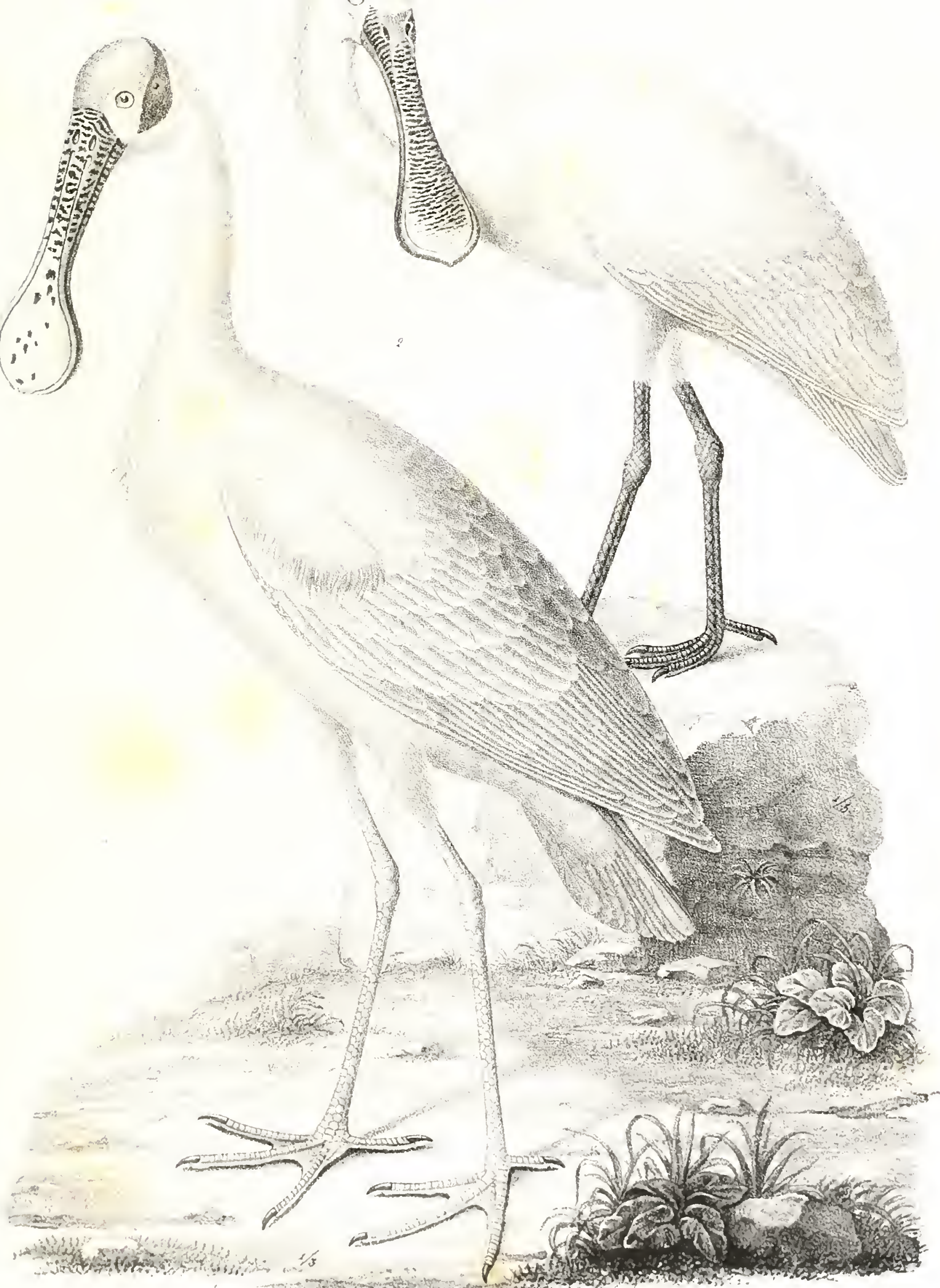

$$
\text { Hejose lenteles }
$$

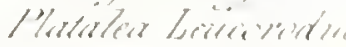

- bovelele bianesher

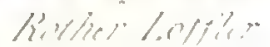

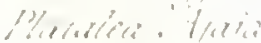

Sompiar rive 

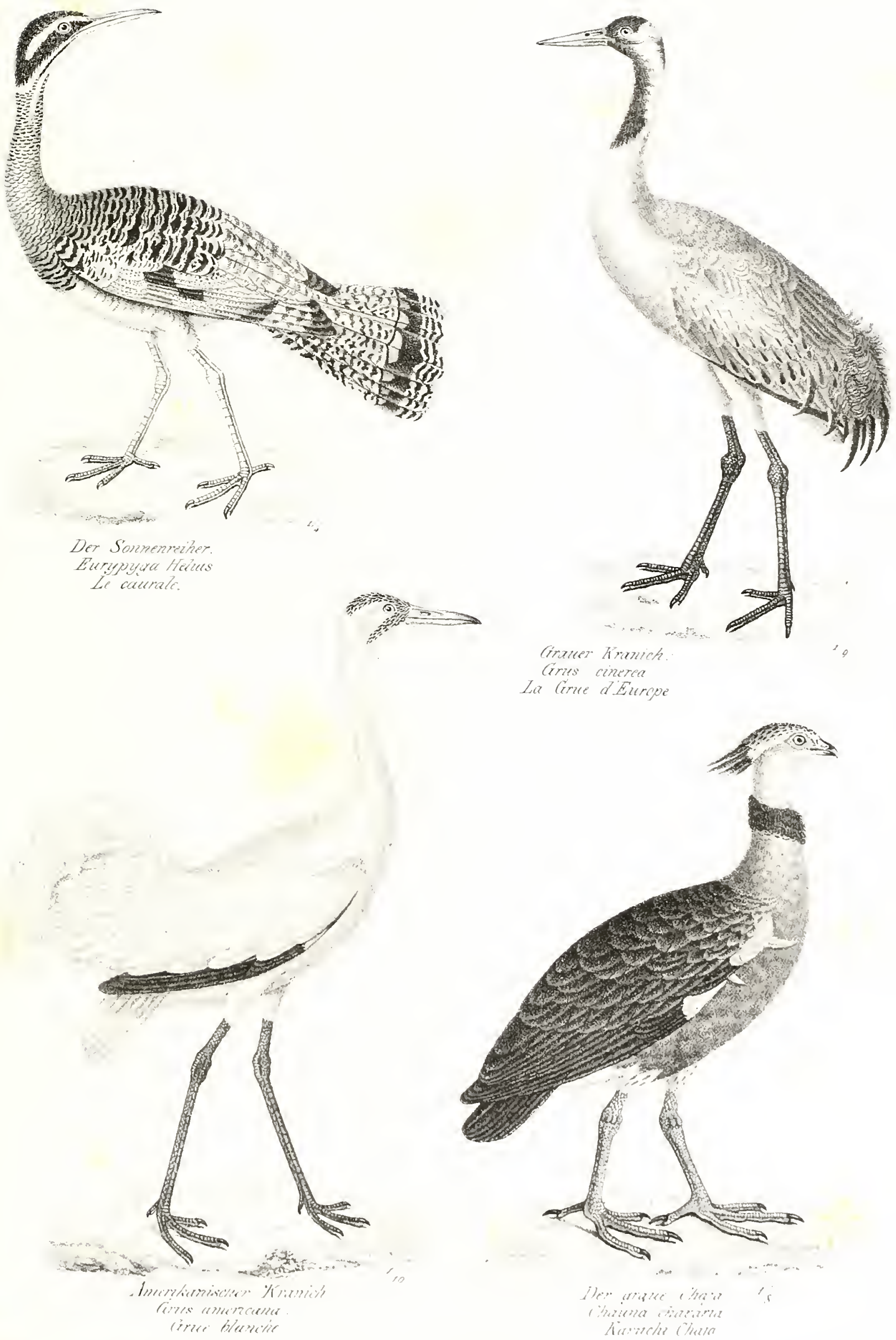


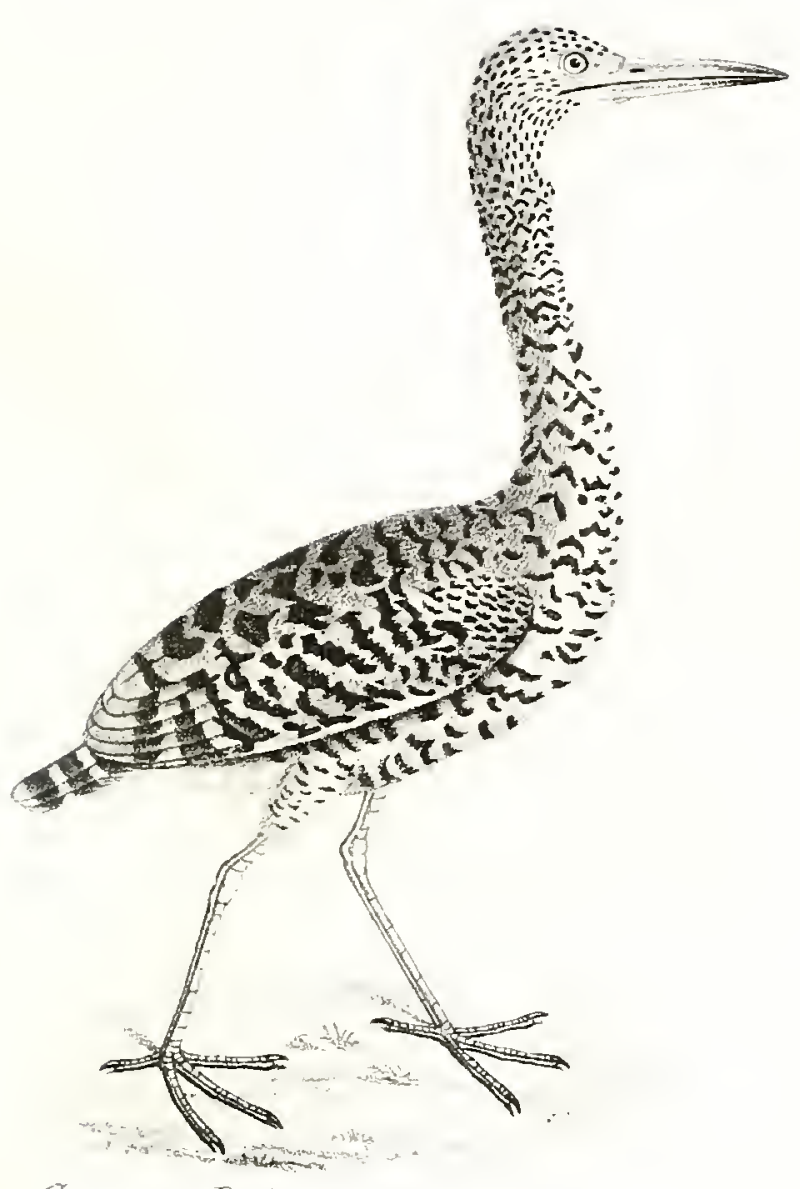

Aezgerrer Reine?

- triter agrima.

Heirue ligre

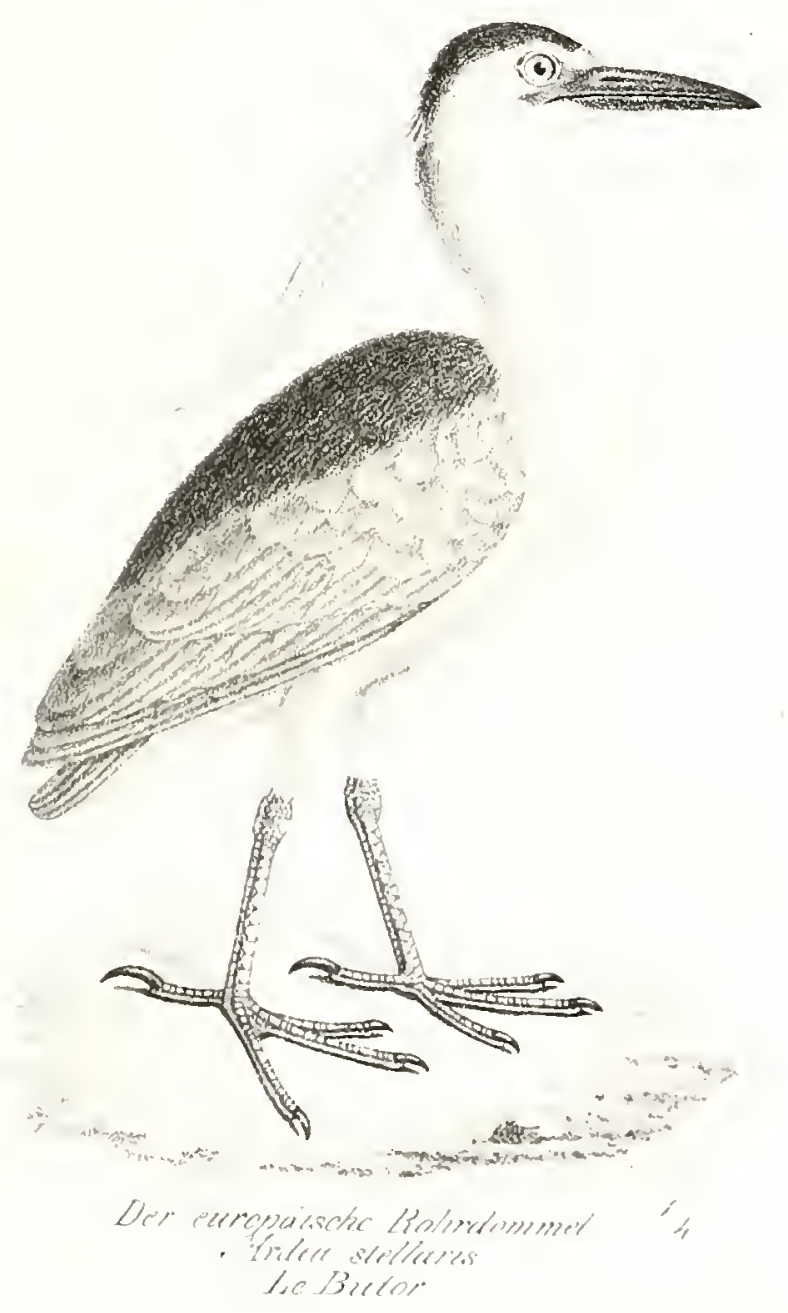

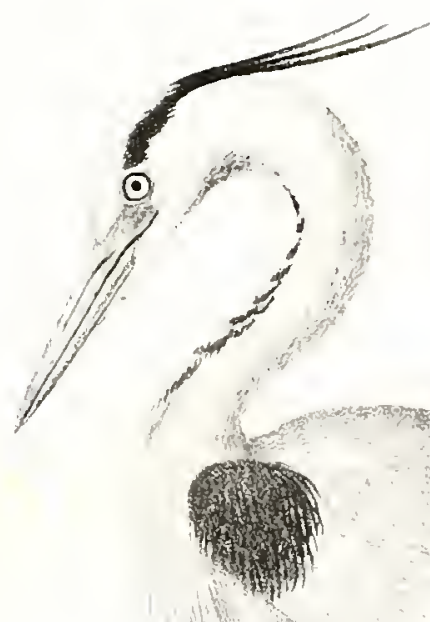

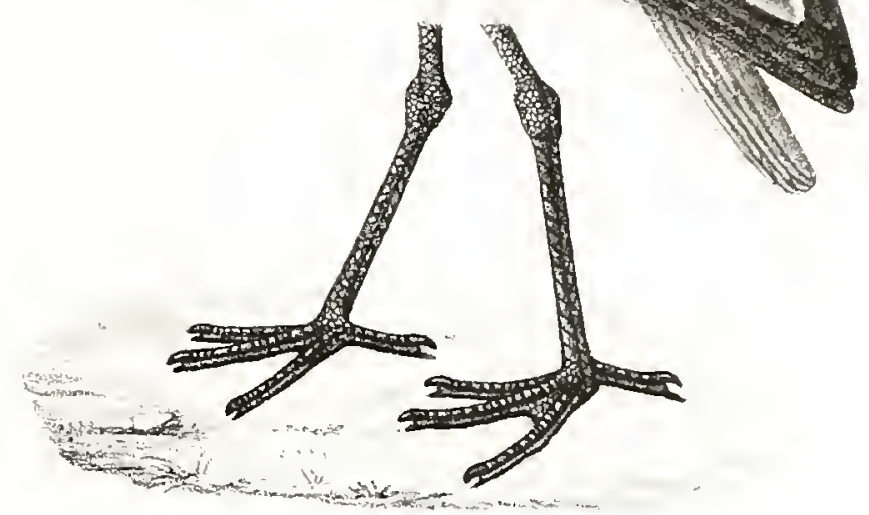

rimerer liever 'o

- Irder comereir

Heros. rempenes

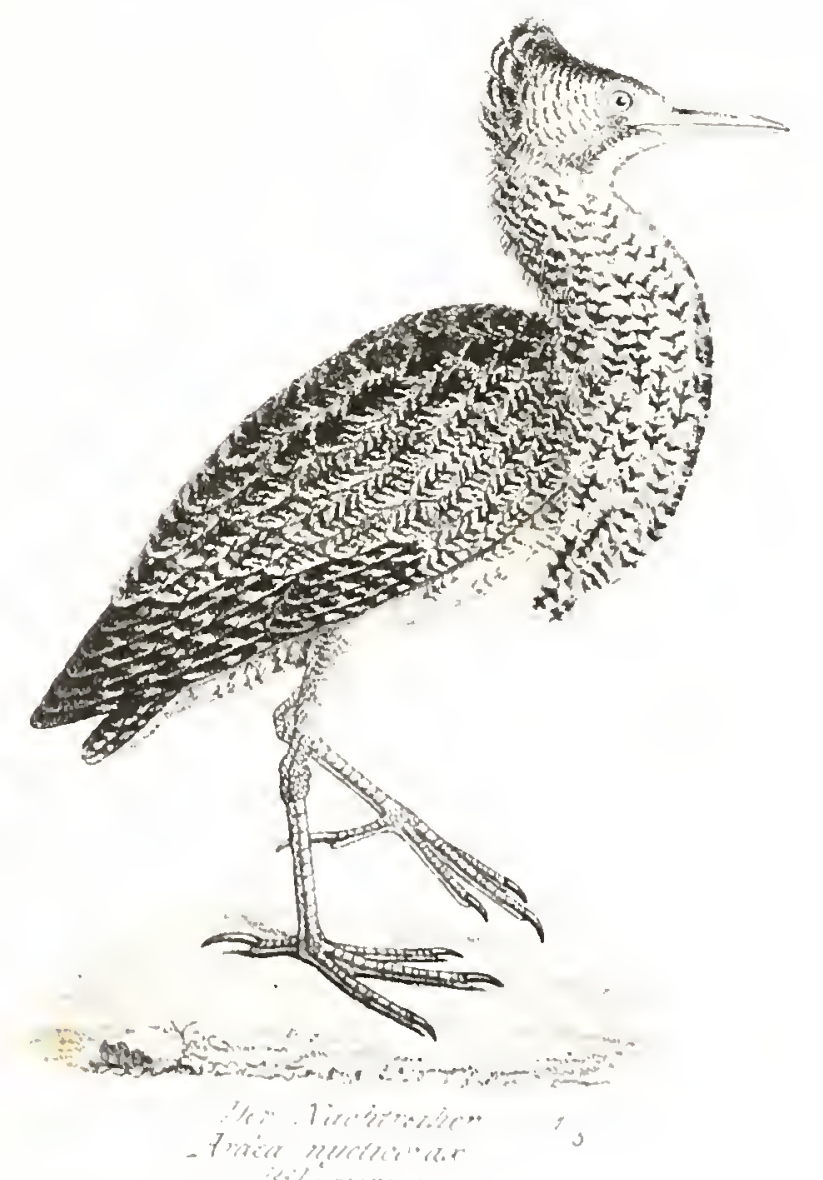




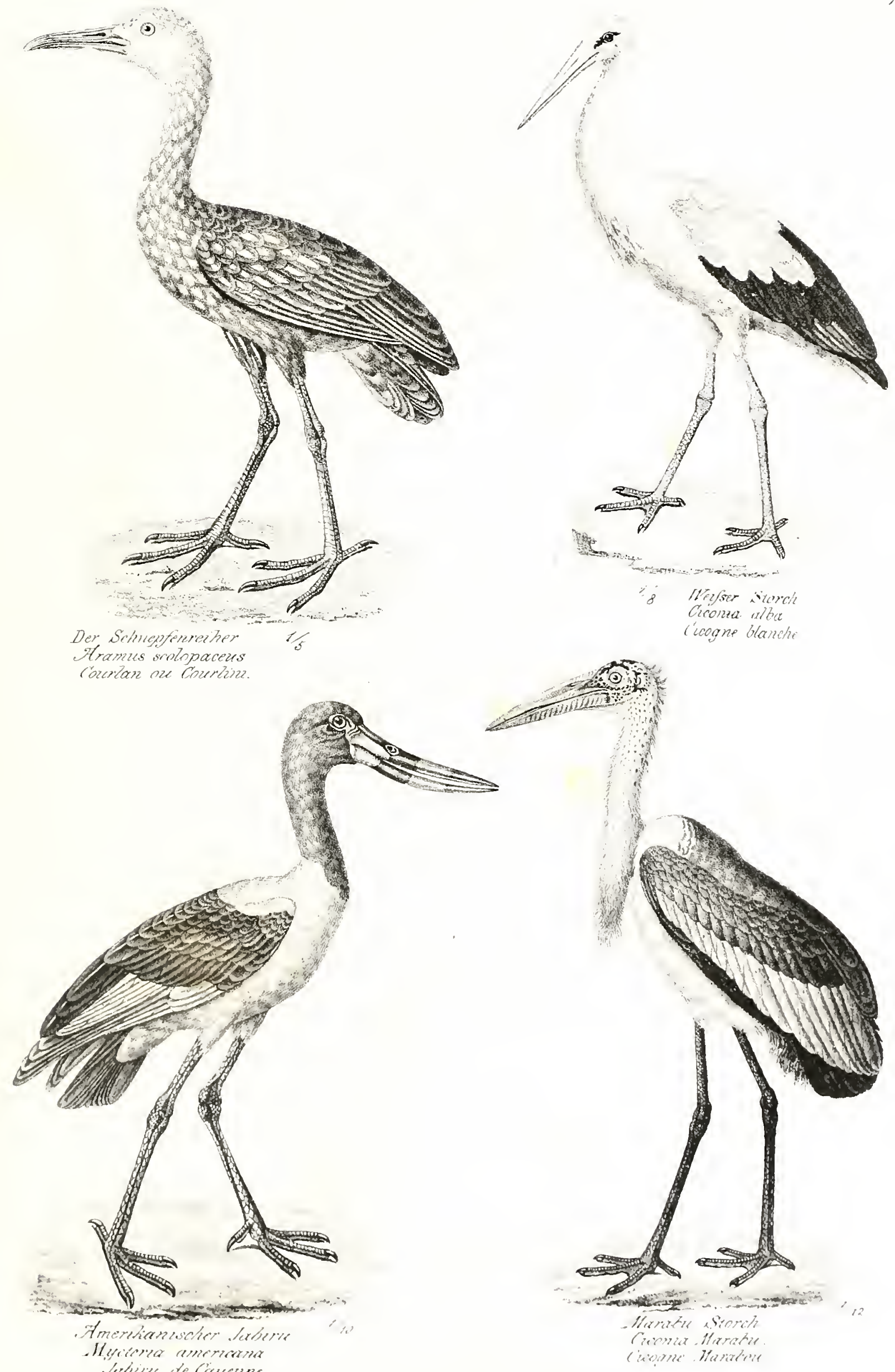

Mrames srolopaceus 


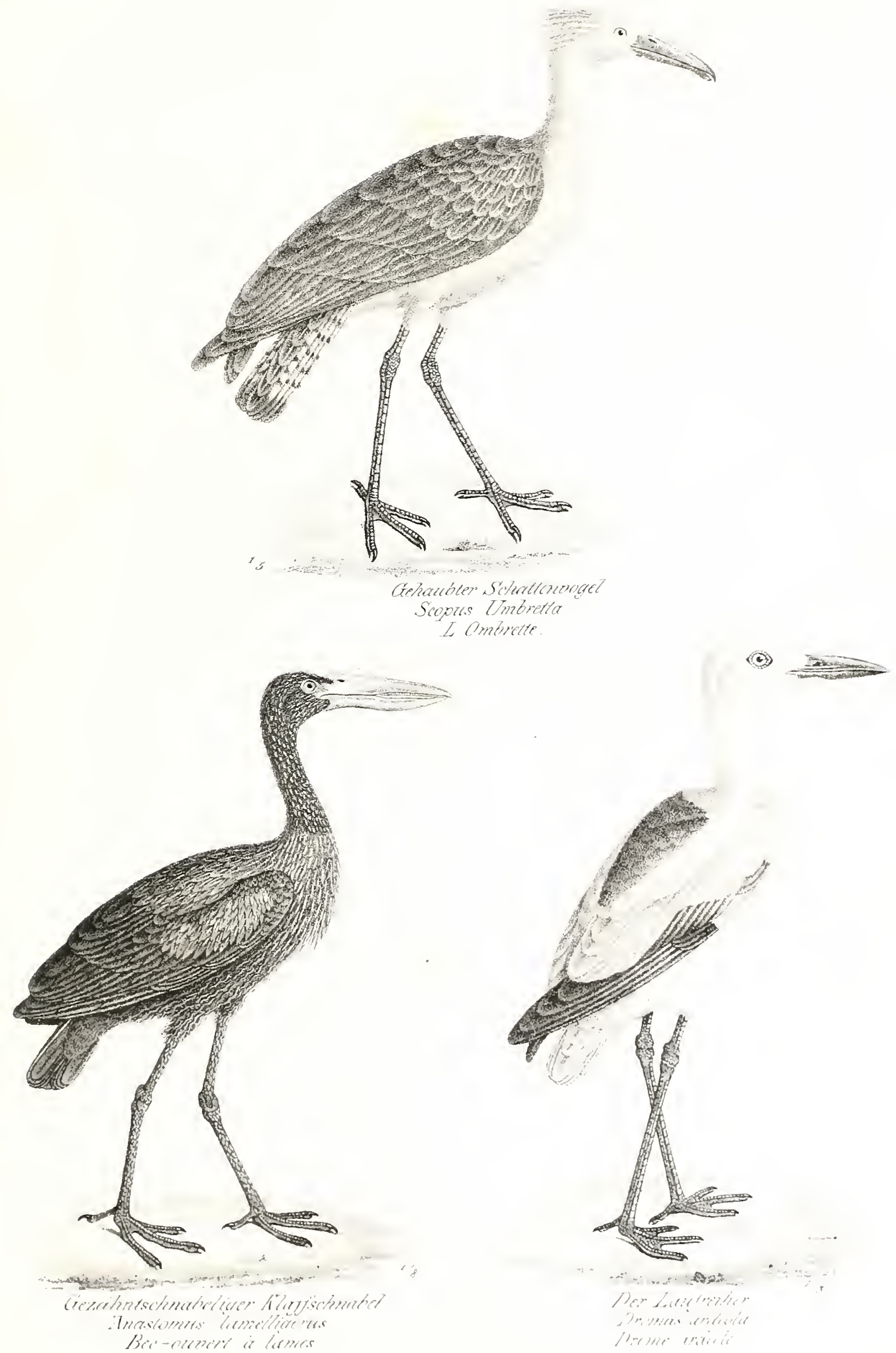



c)
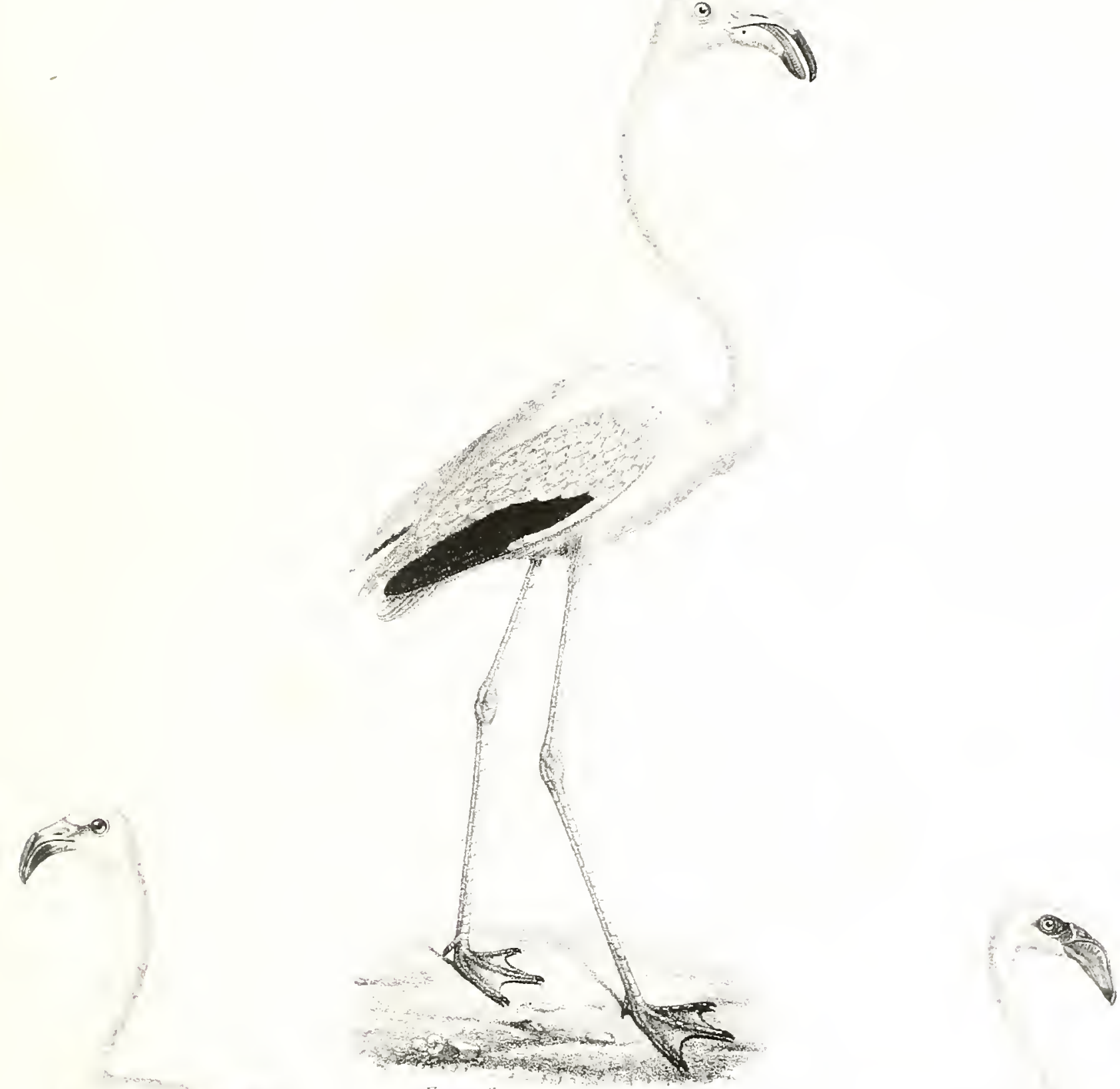

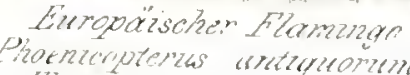

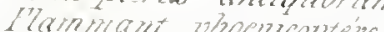
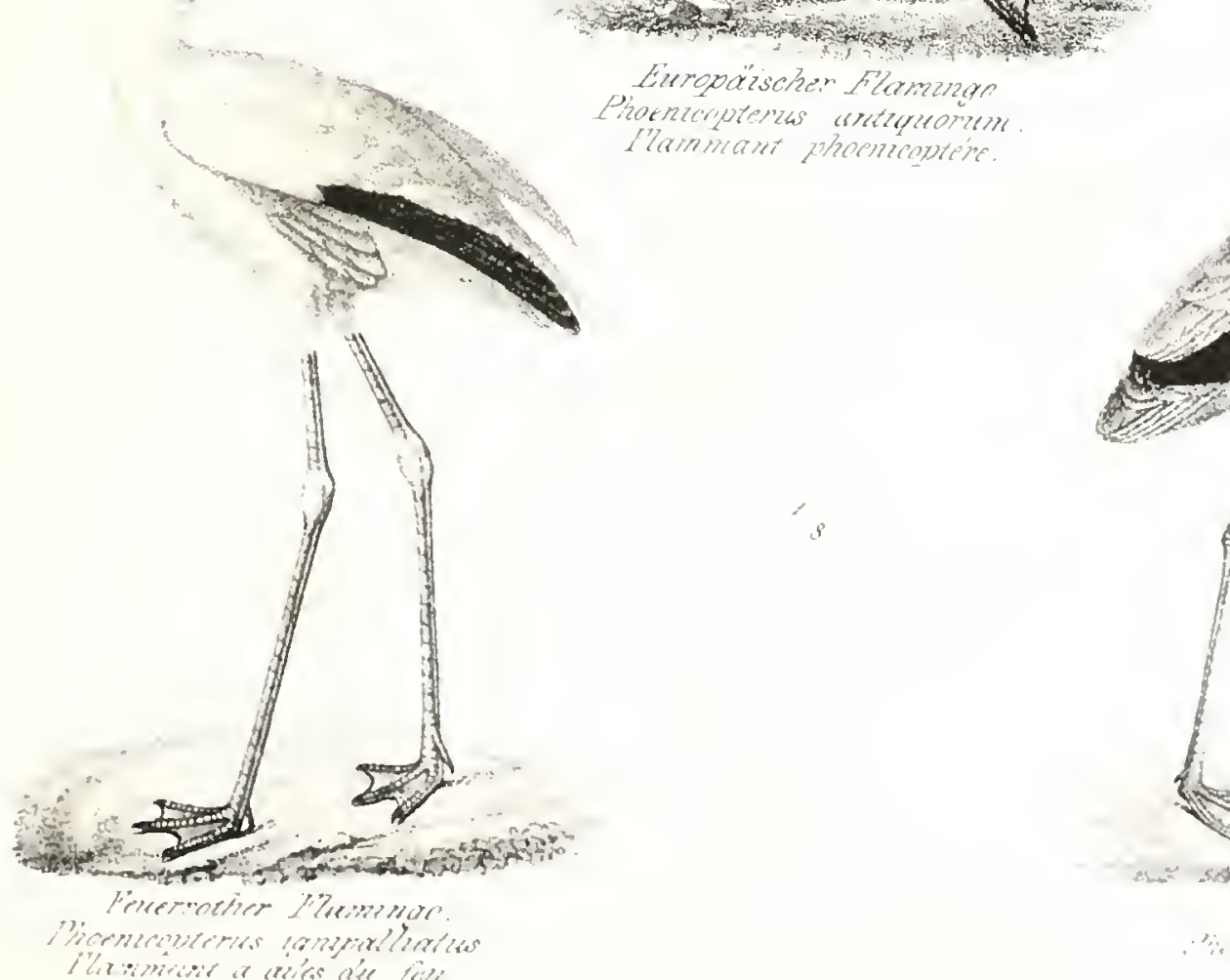


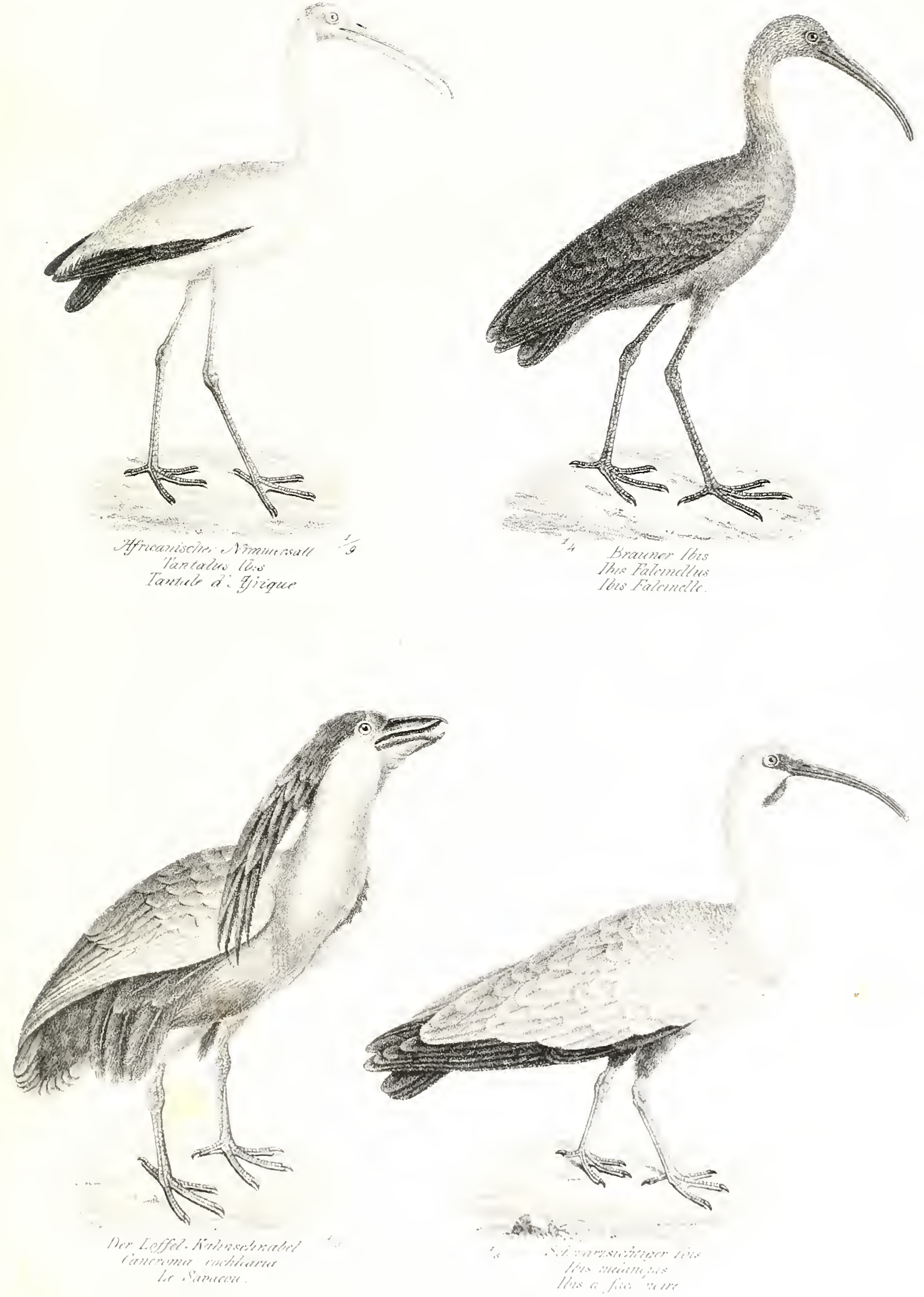


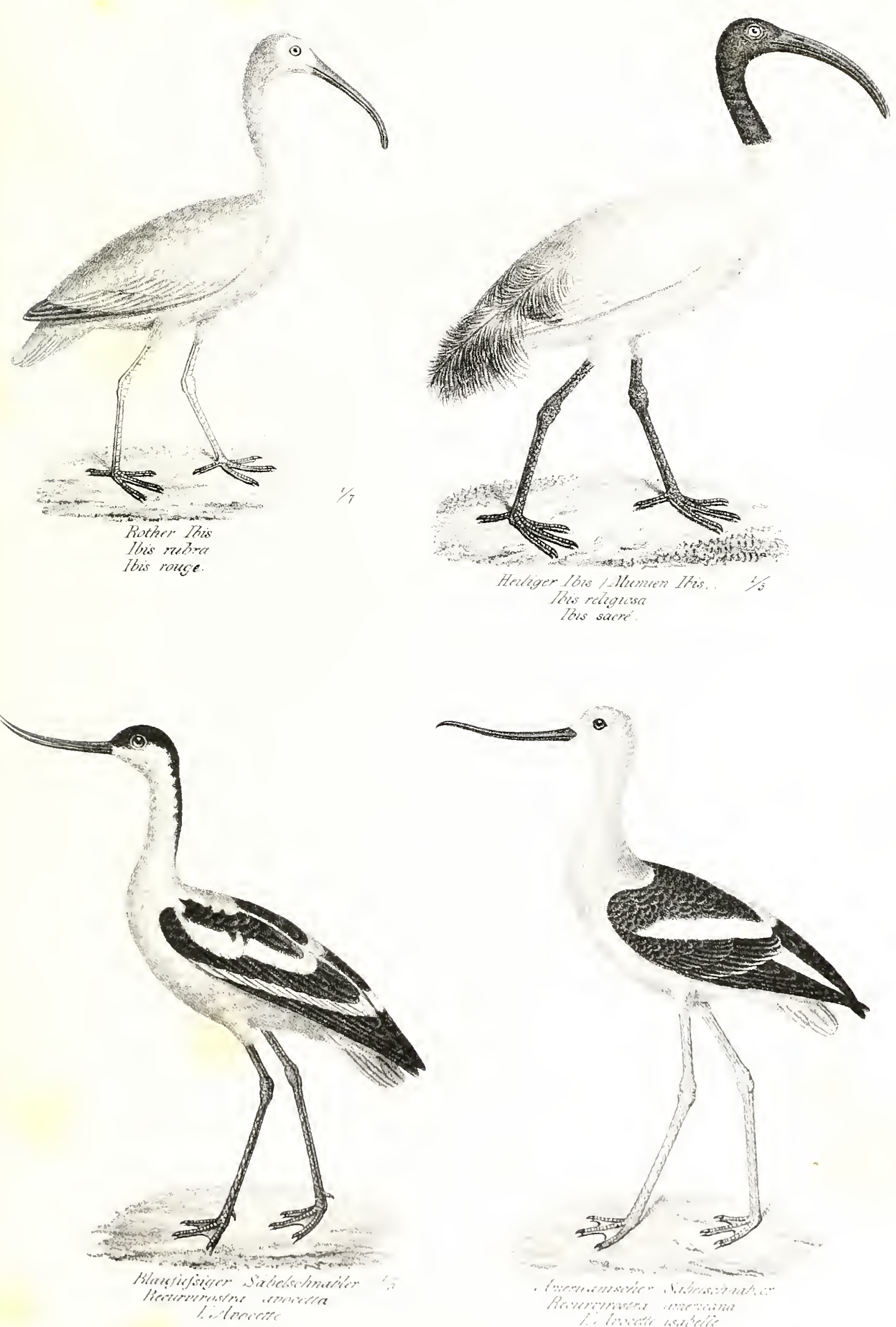

0

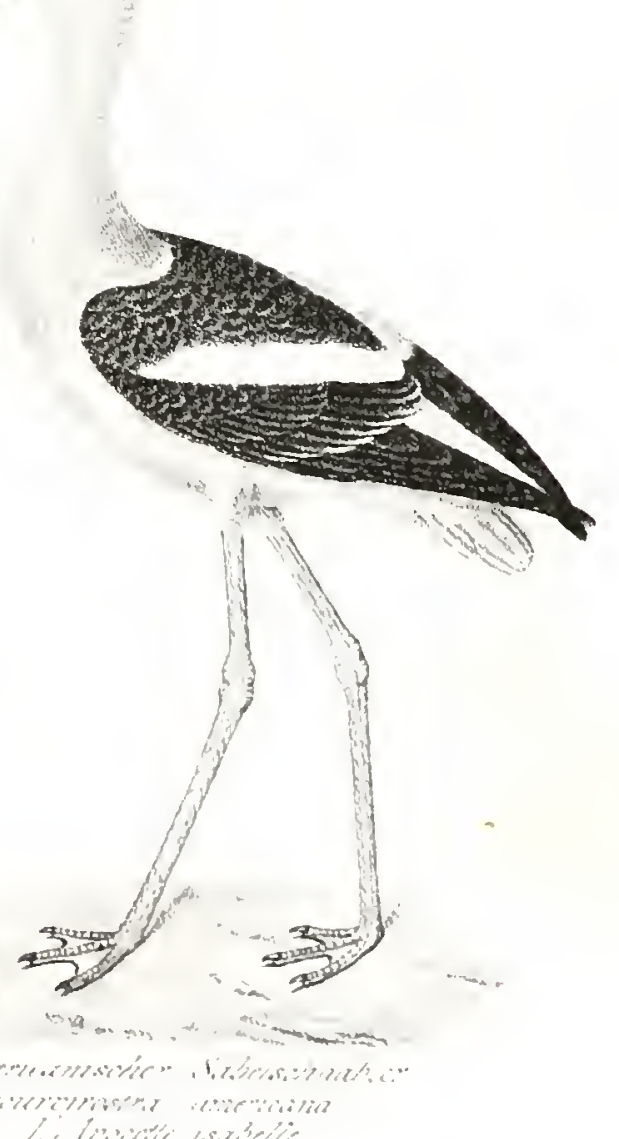





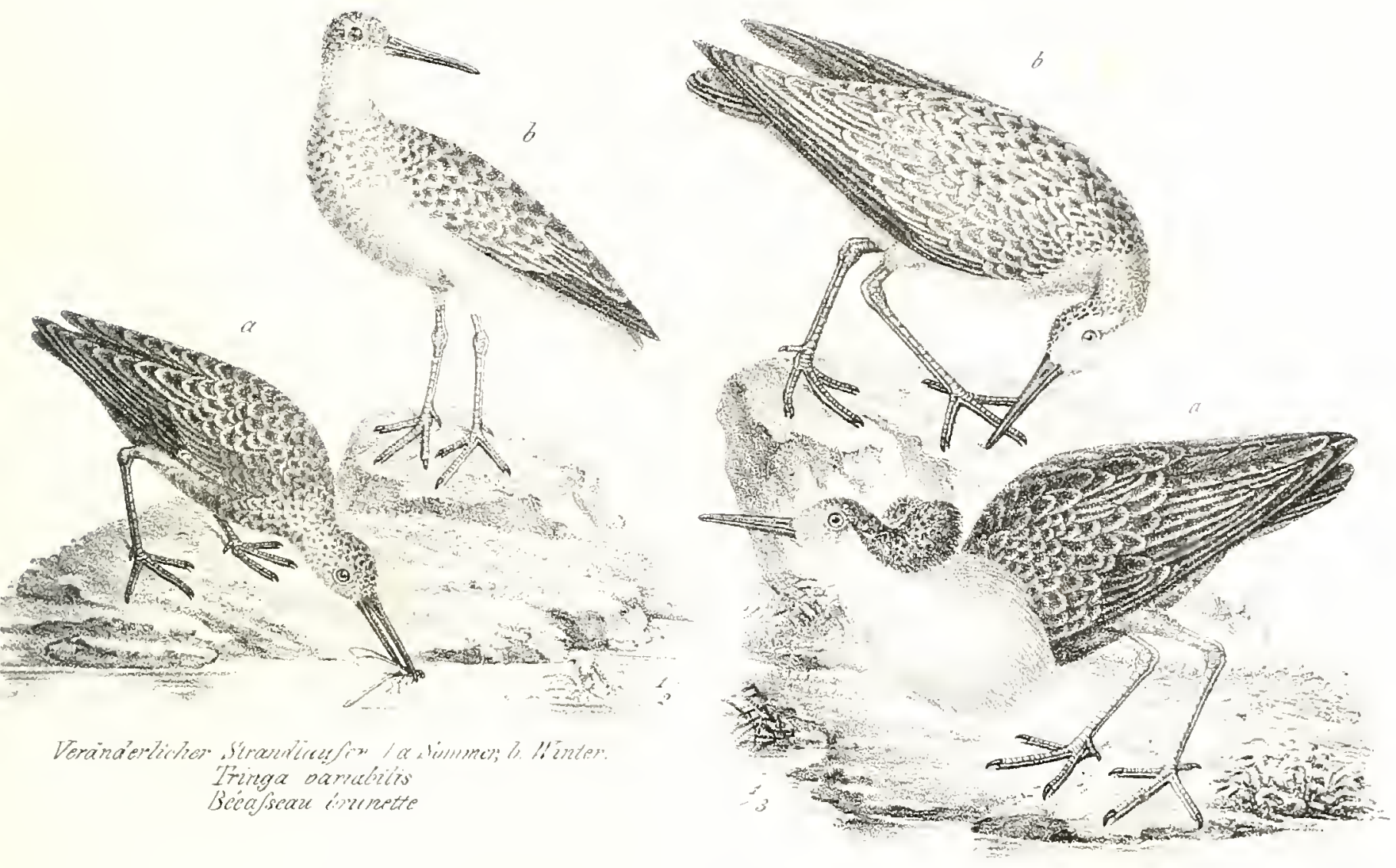

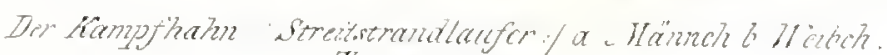
Irrnga pugner. becalseaz combuteant
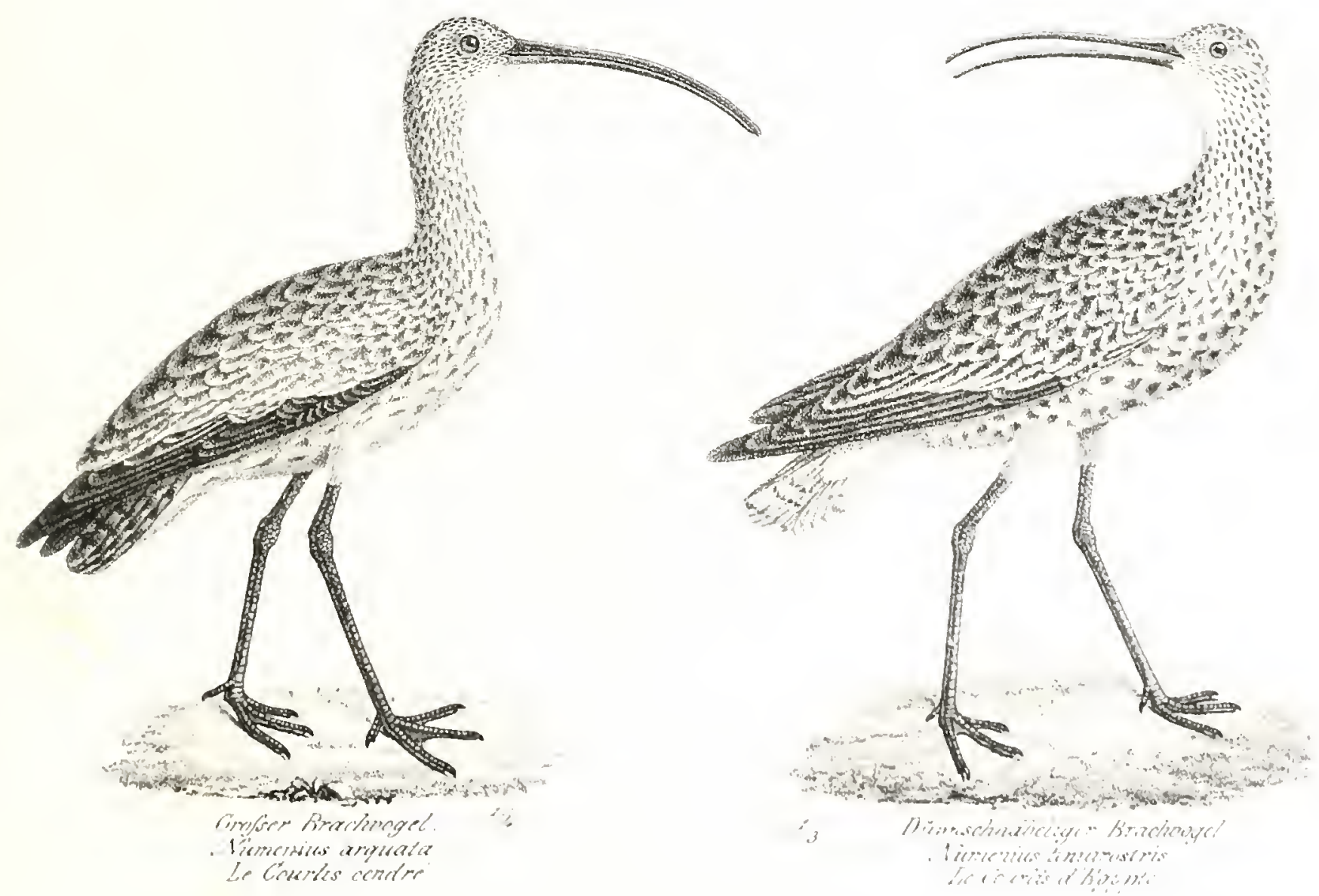

$19 \%$
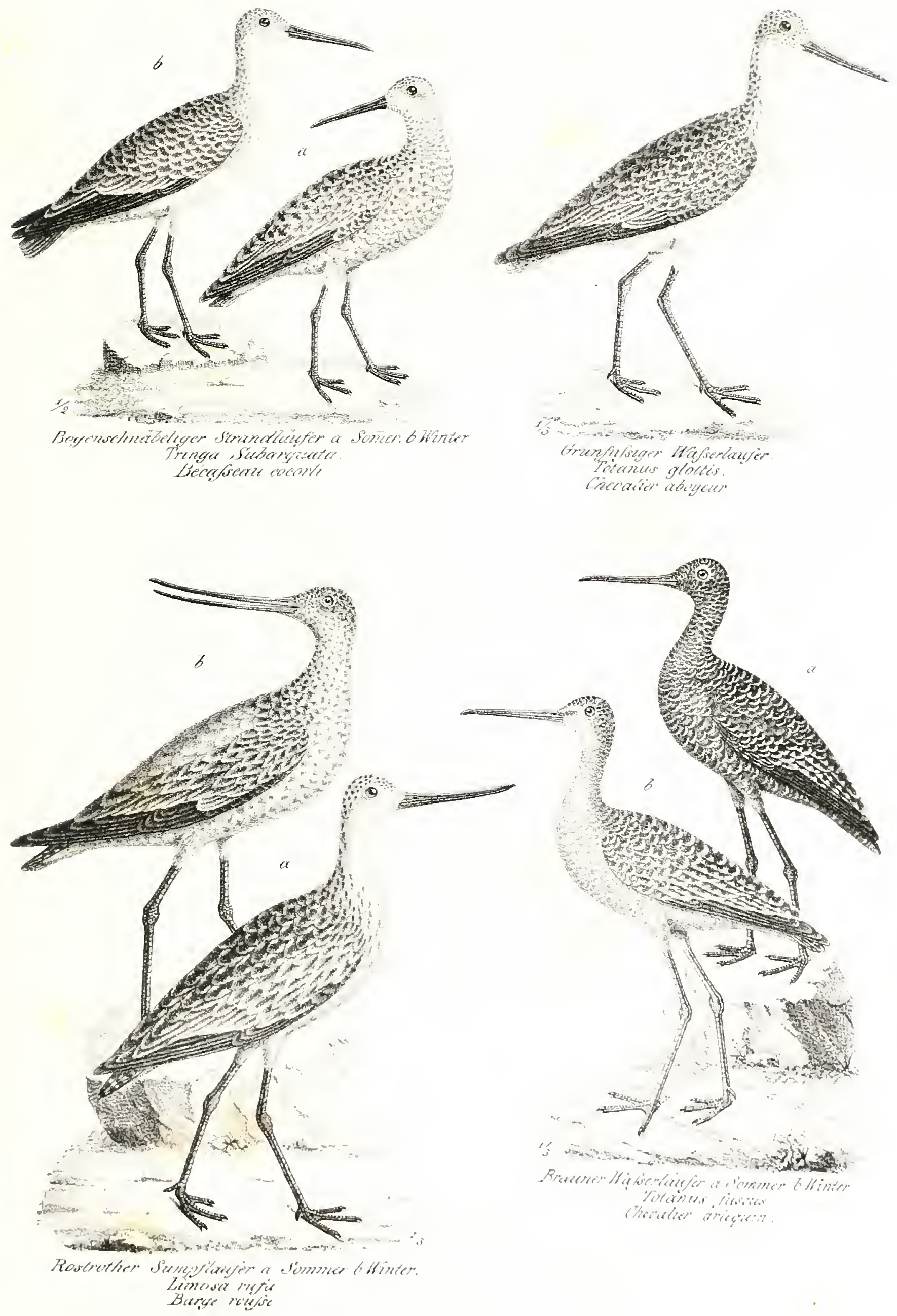


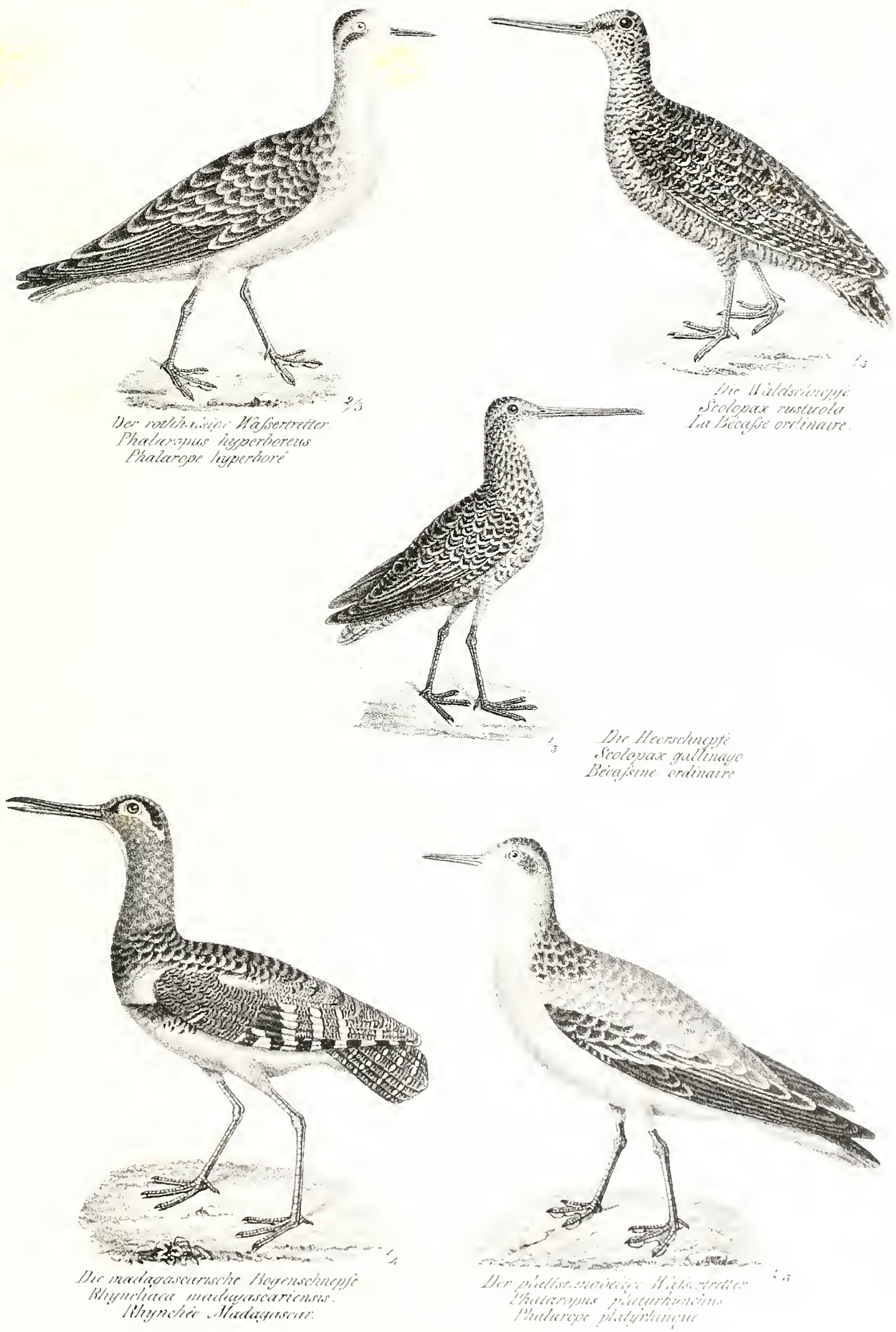



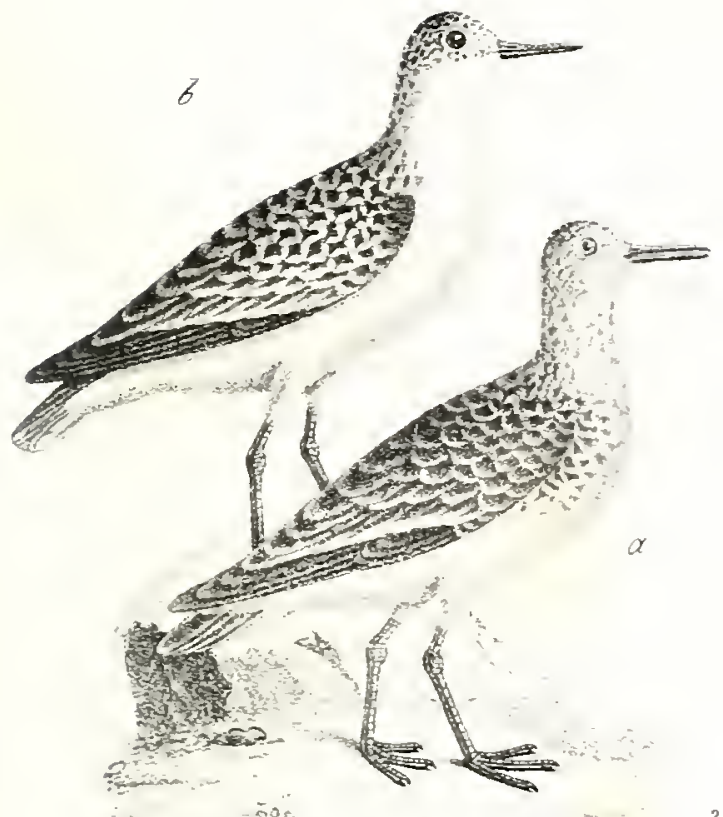

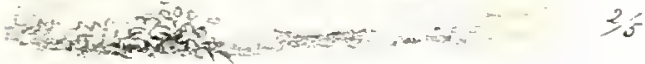

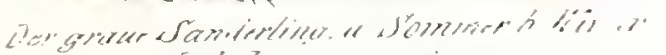

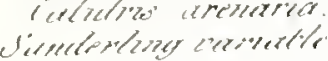

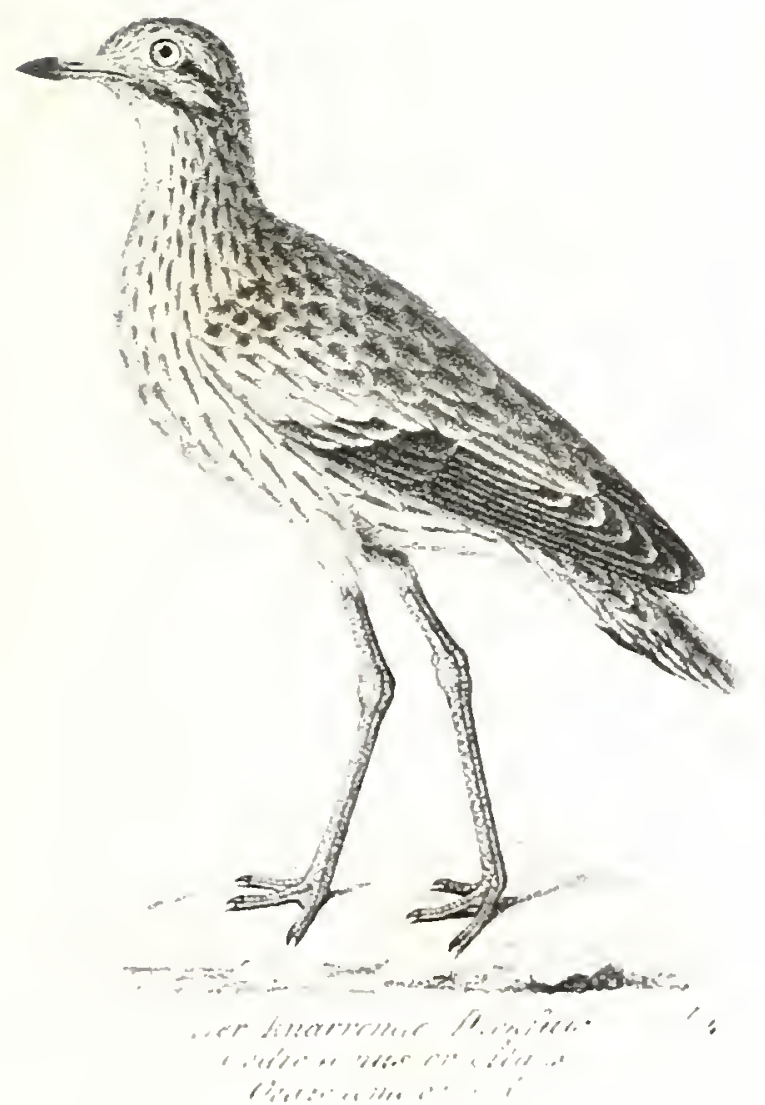

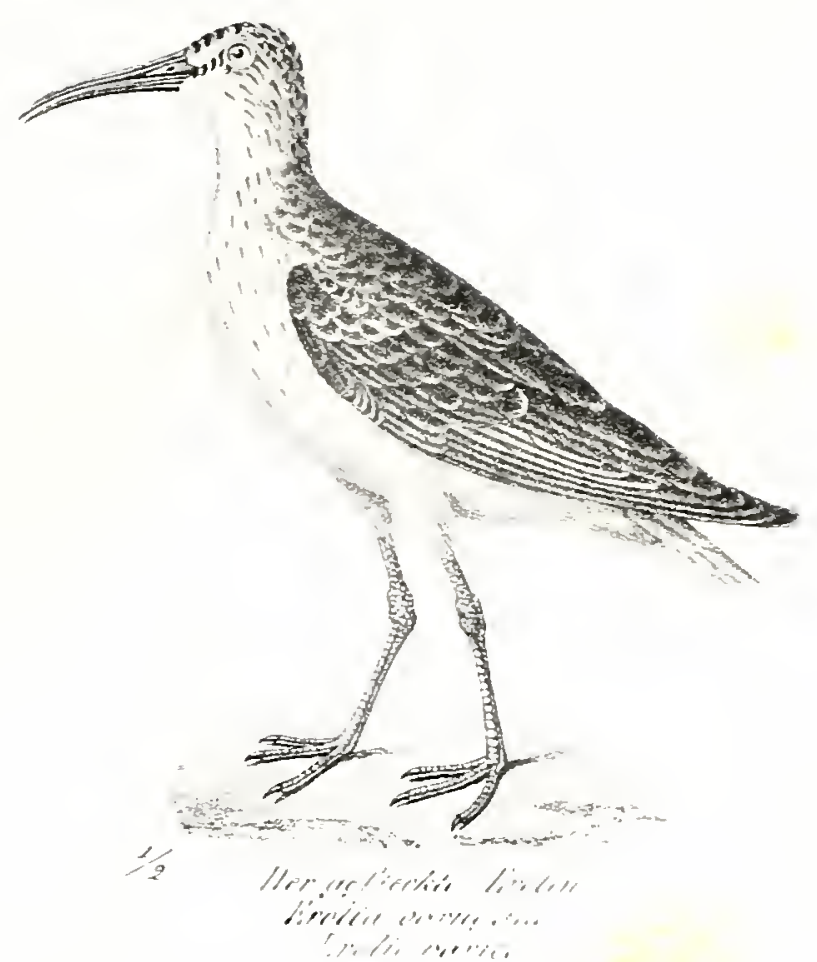

$\frac{1}{20}$

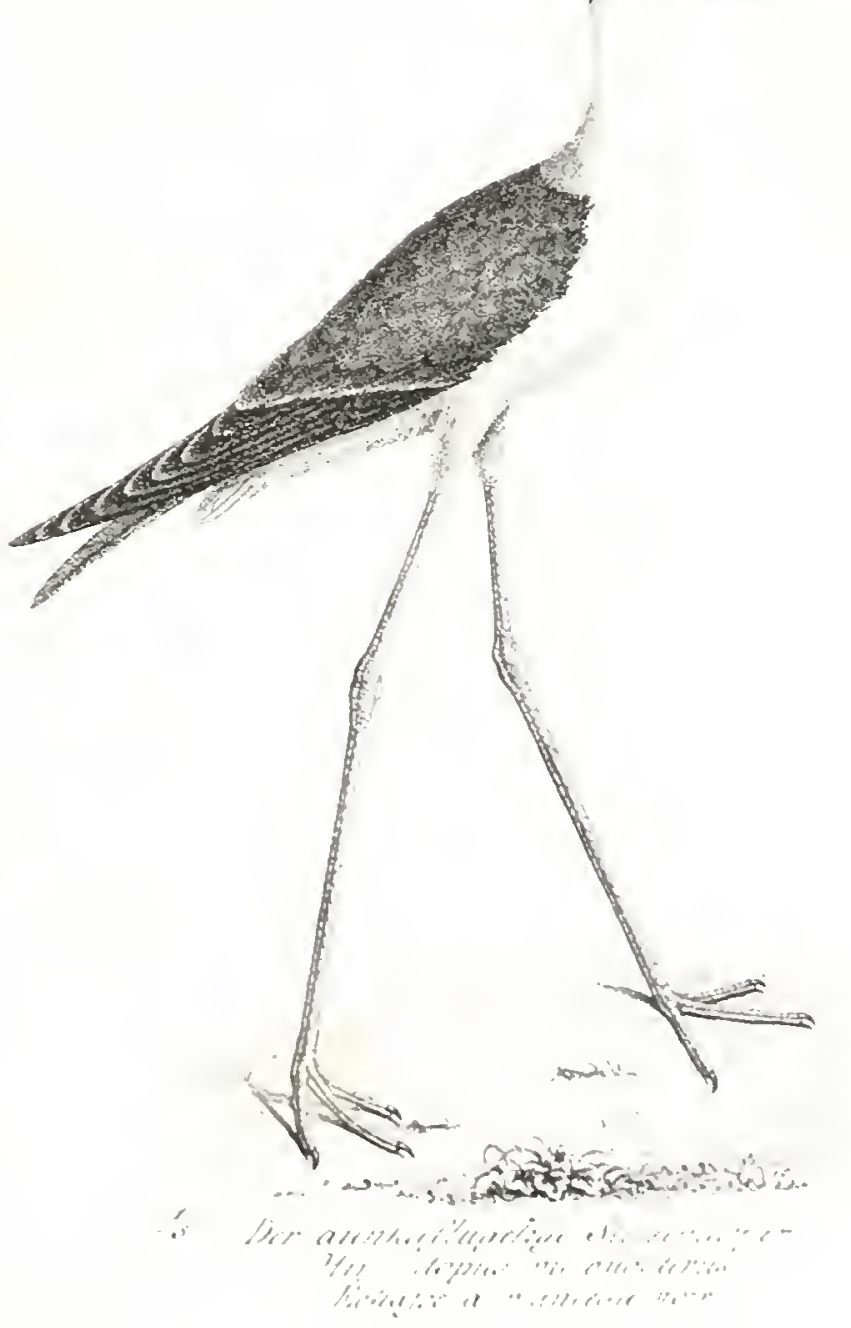




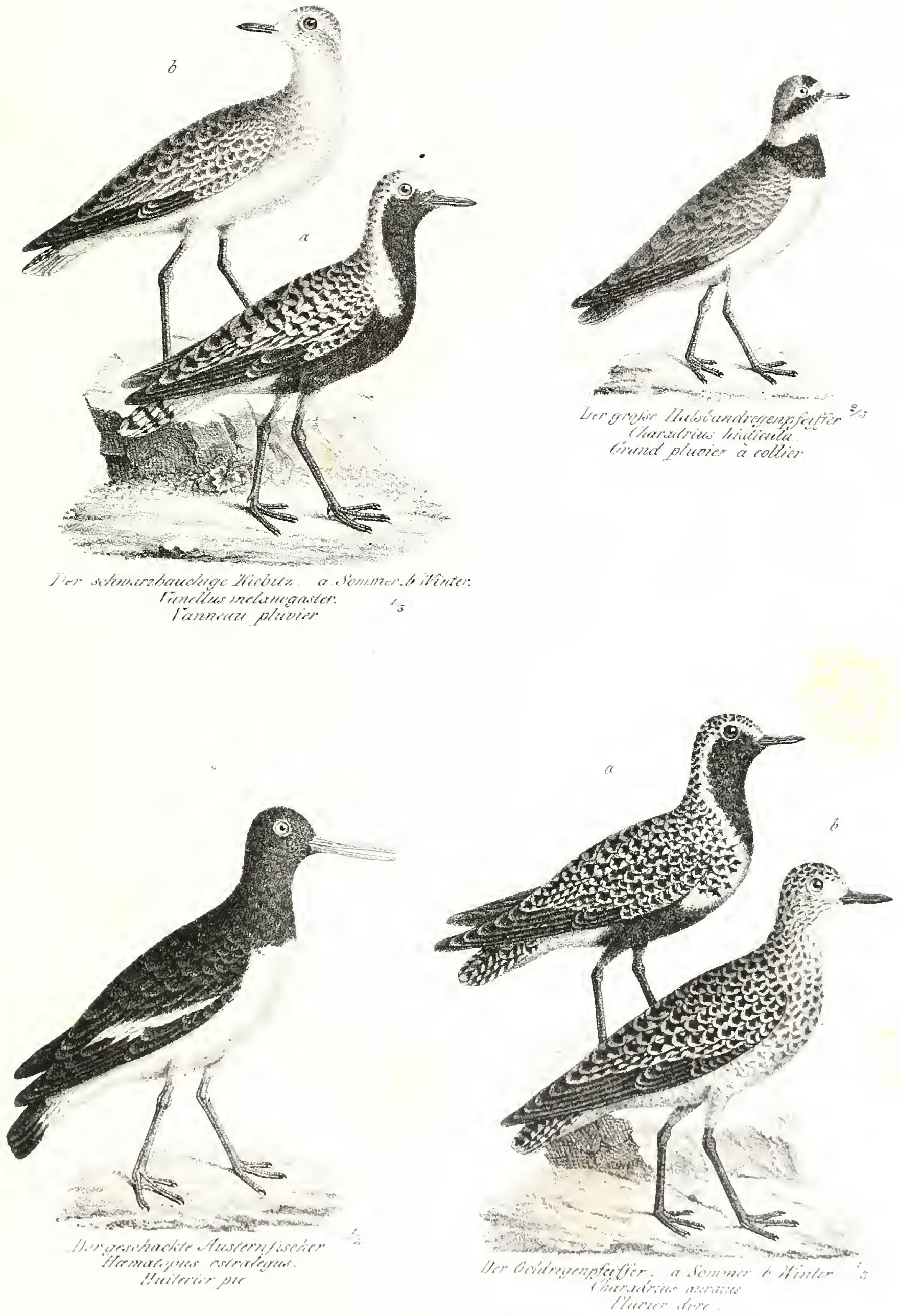


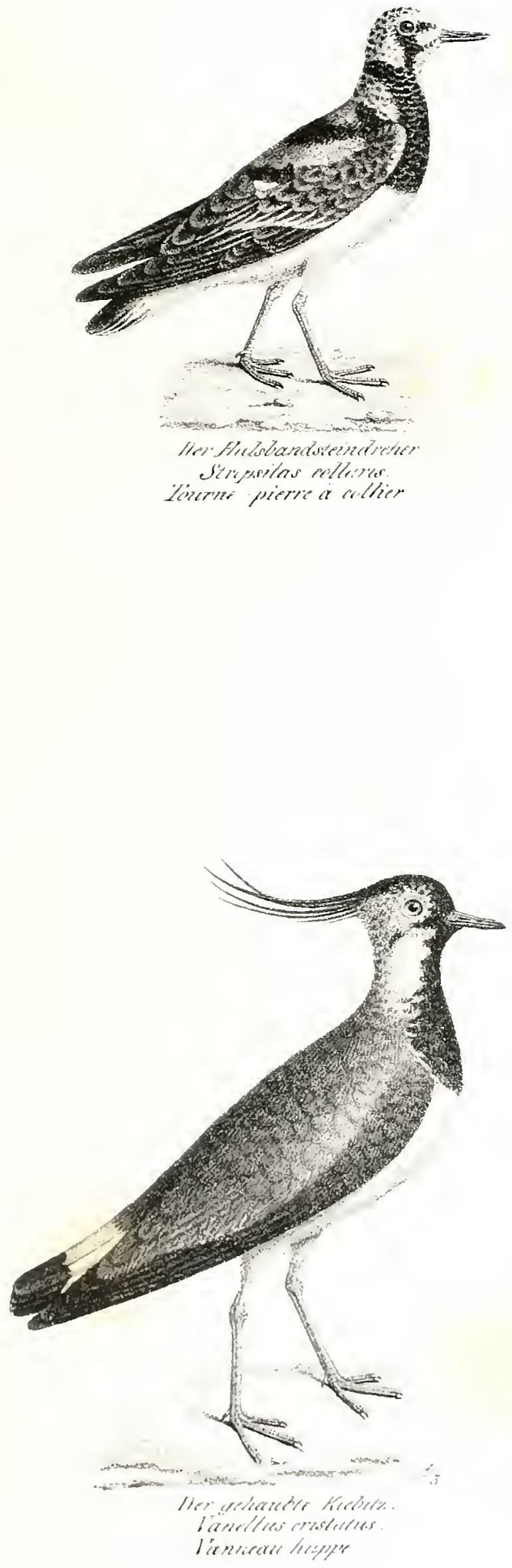
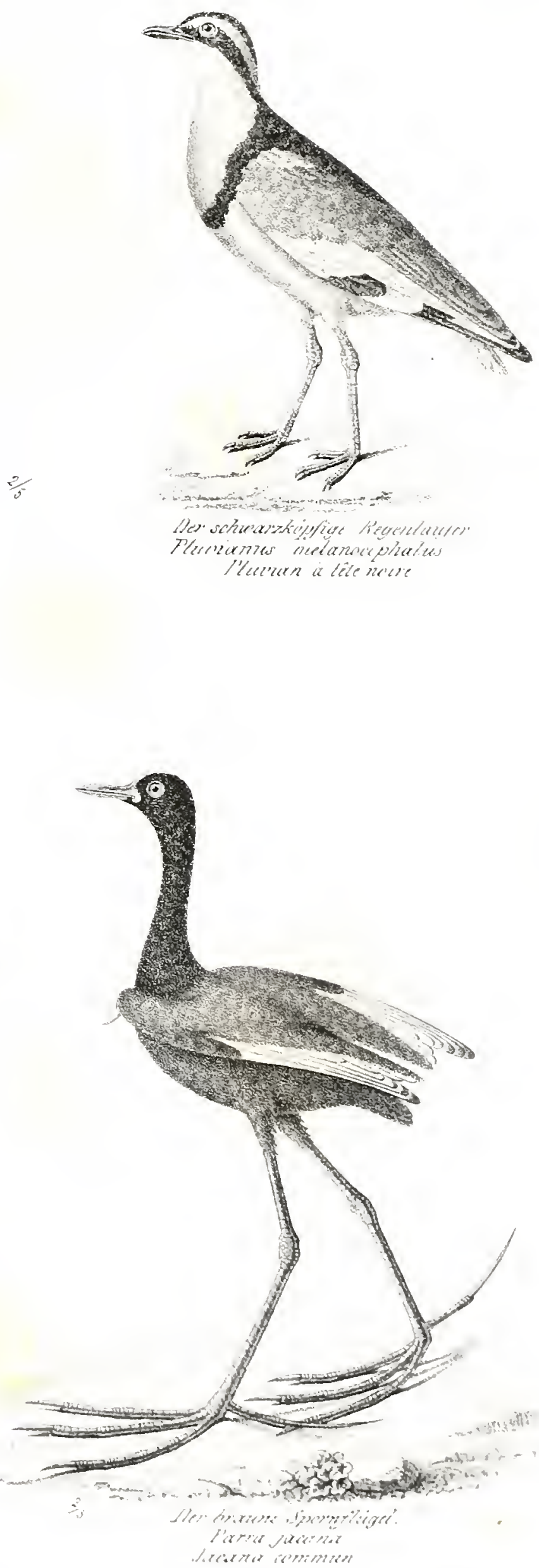


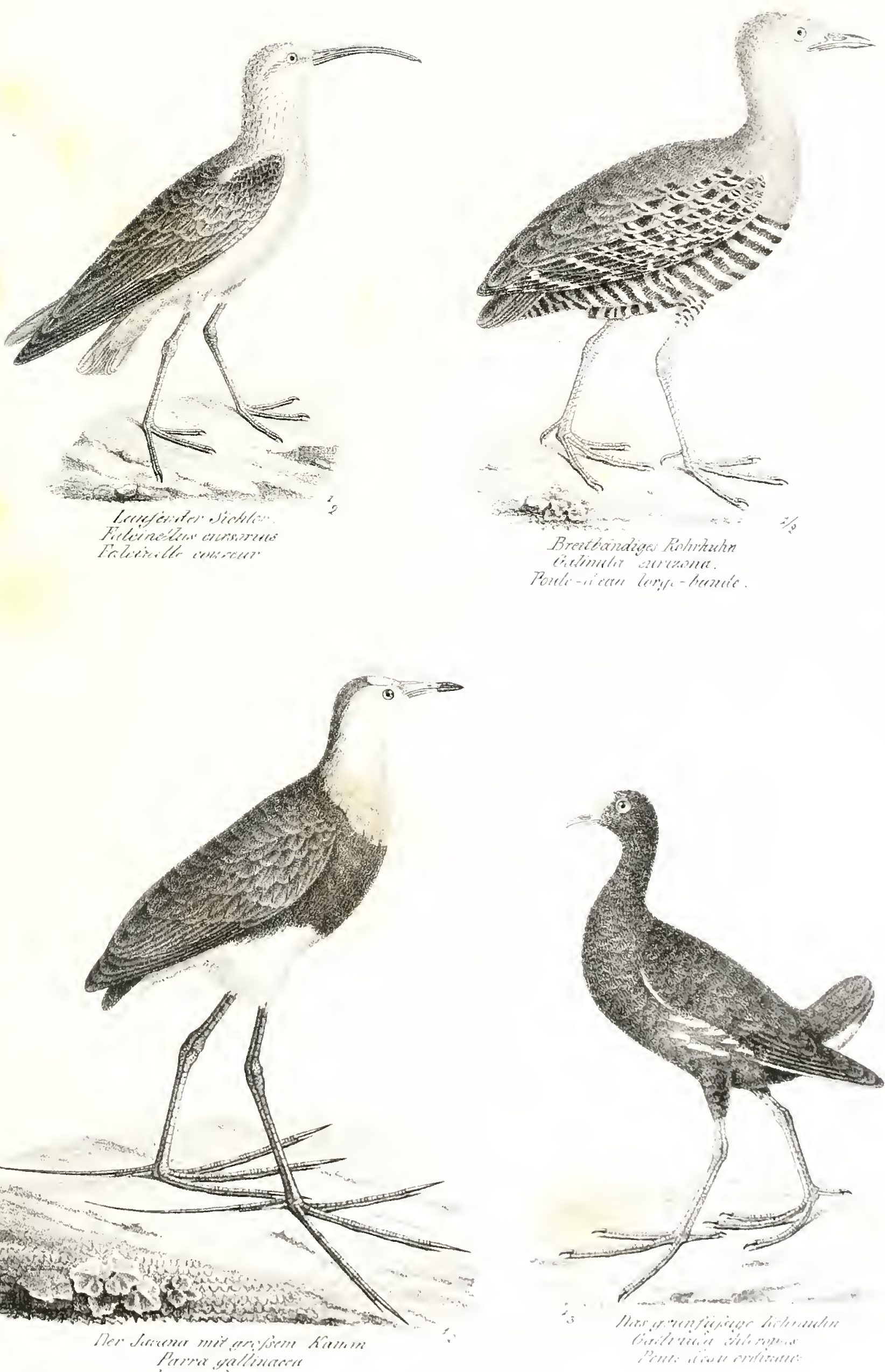

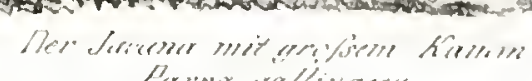
Perrese sollisureres 



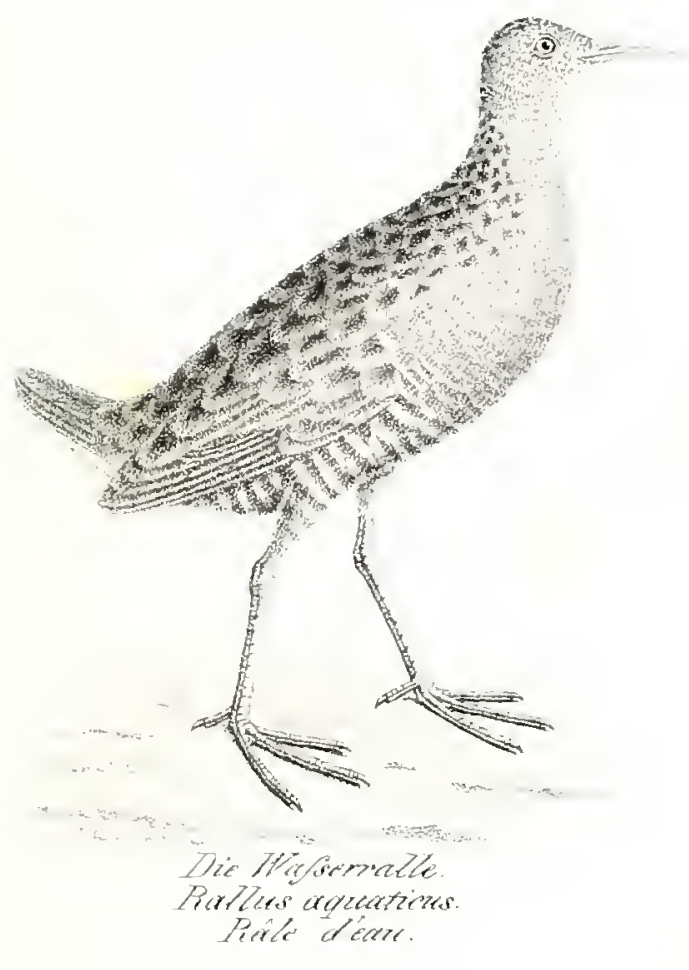

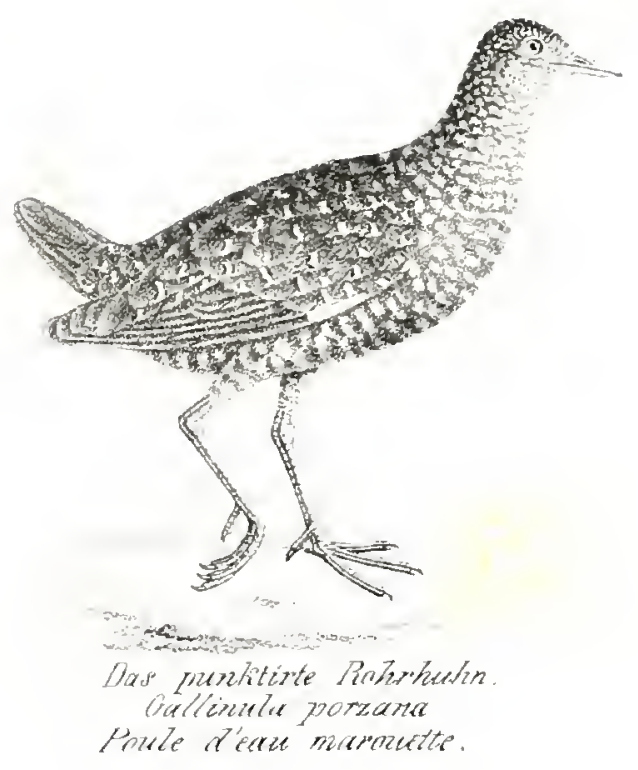
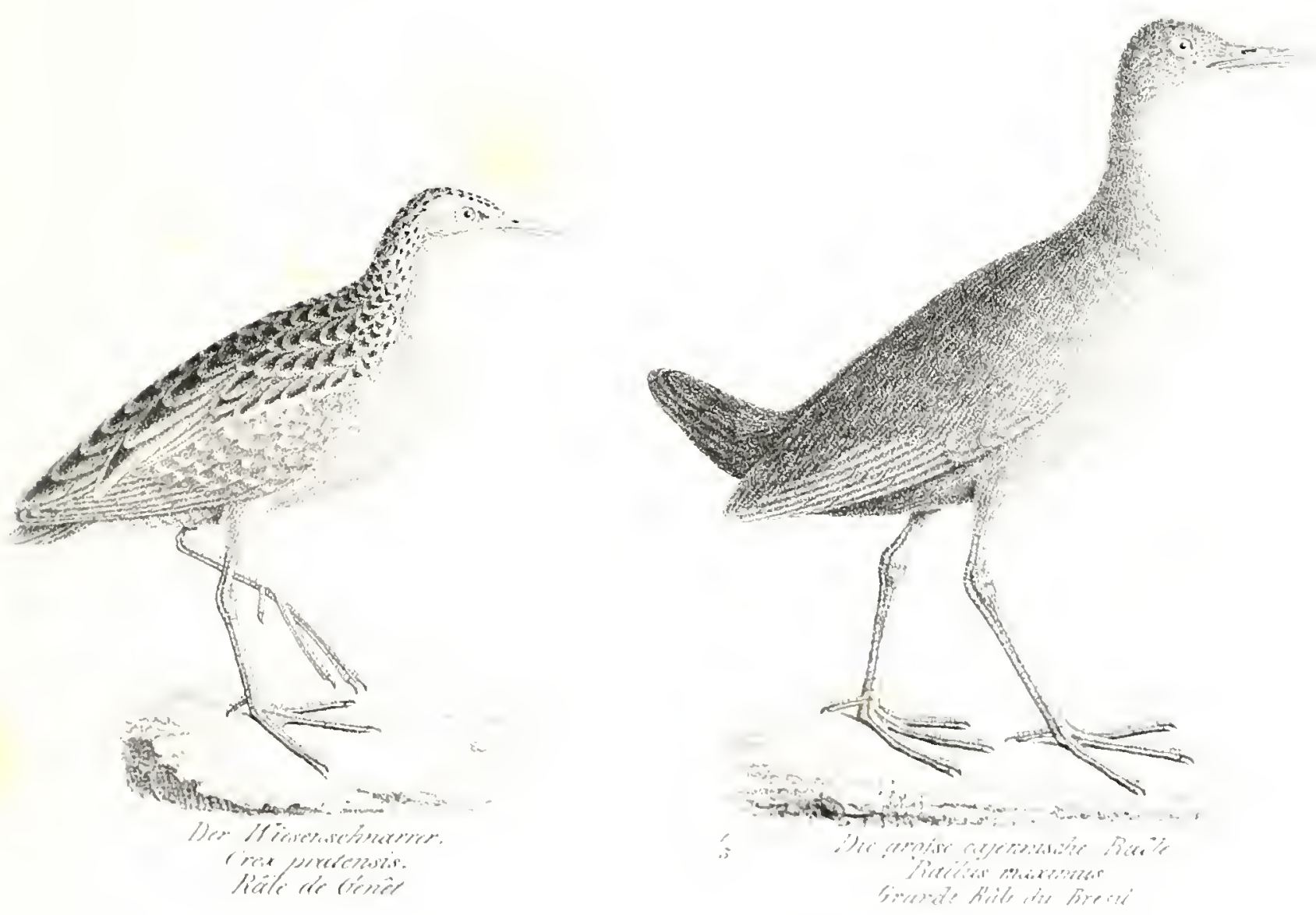


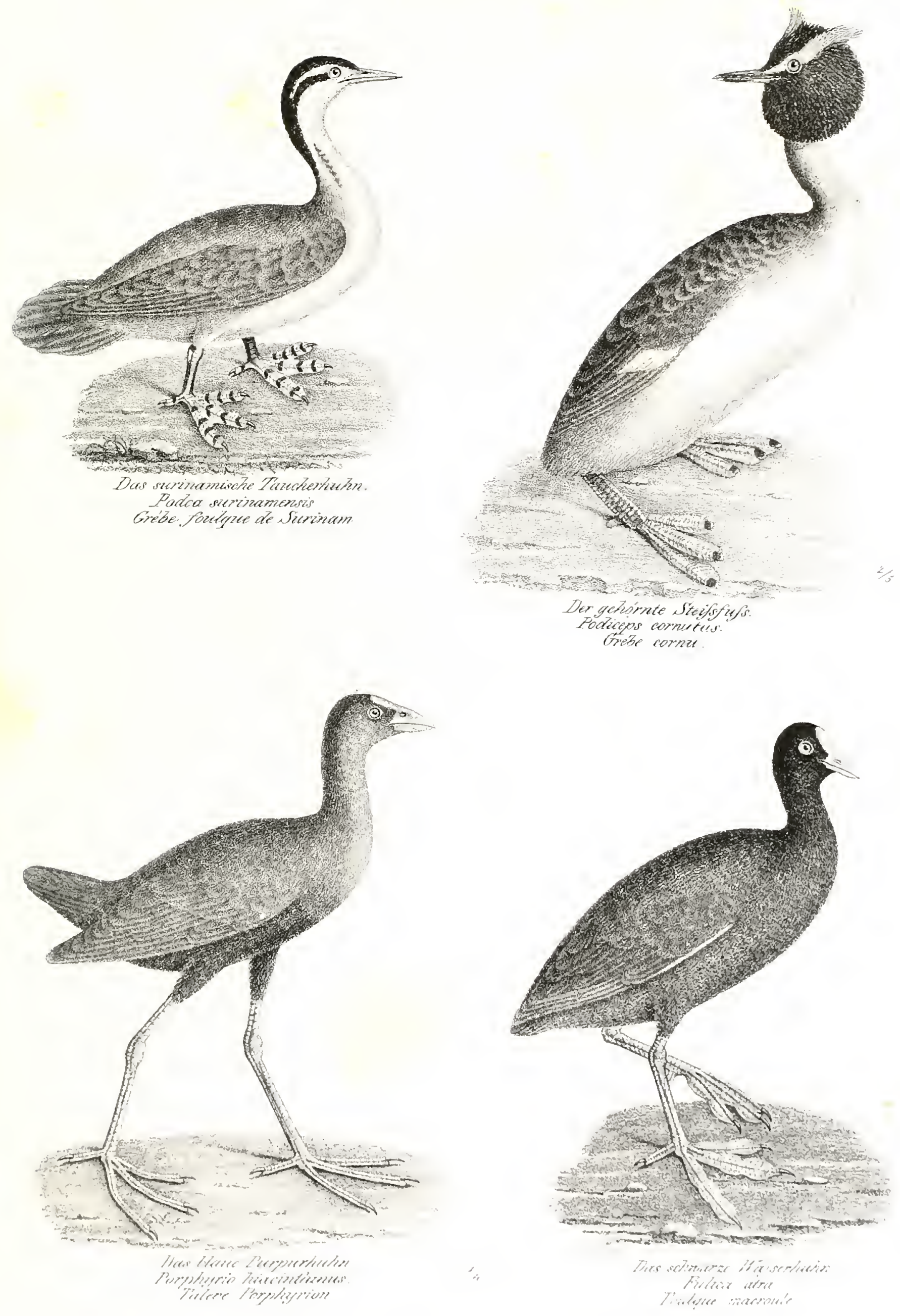



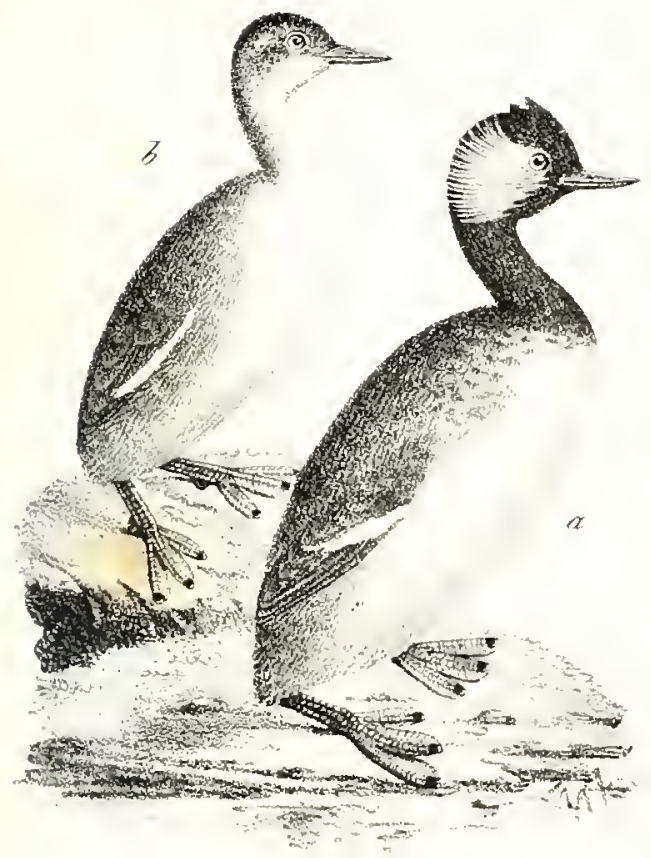

Der Ahrensteiffuf is a Alt. B Jund Podicges aurtits. Griche orcillos.

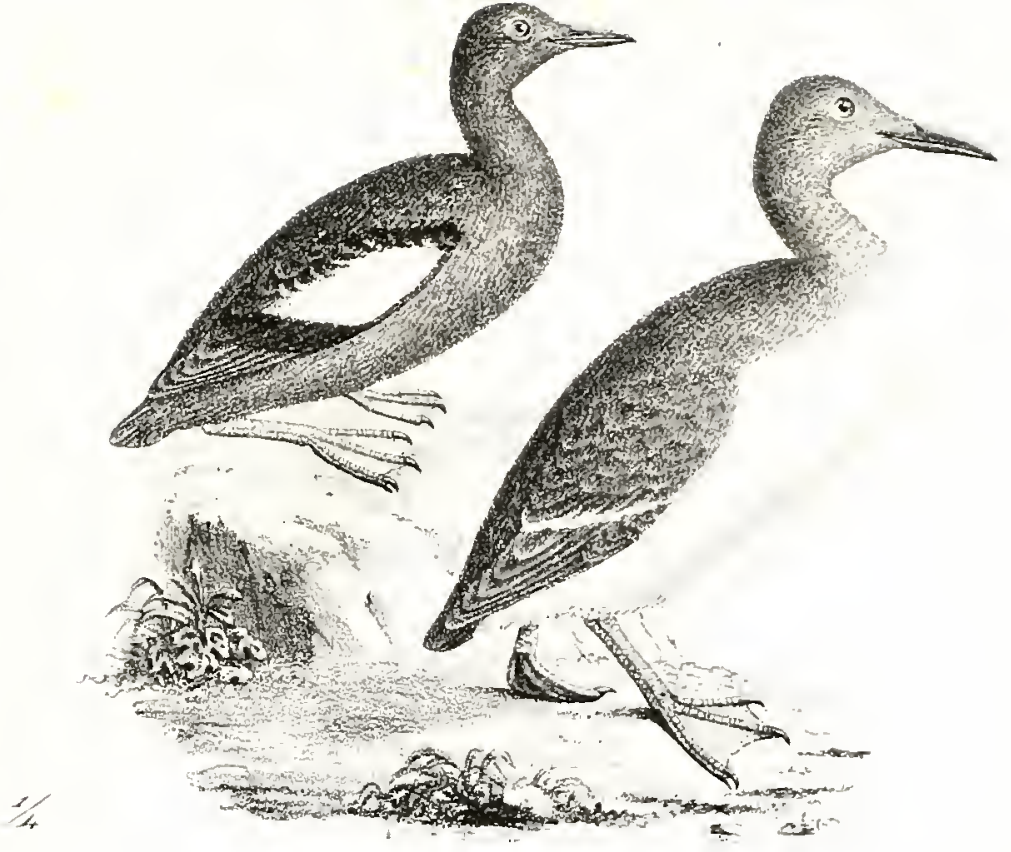

Die dermone Lurmpre.

Tiria grylle.

freitlemec ì mirory blens

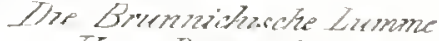
Eirue Brompusit Cuilternot de Braturush
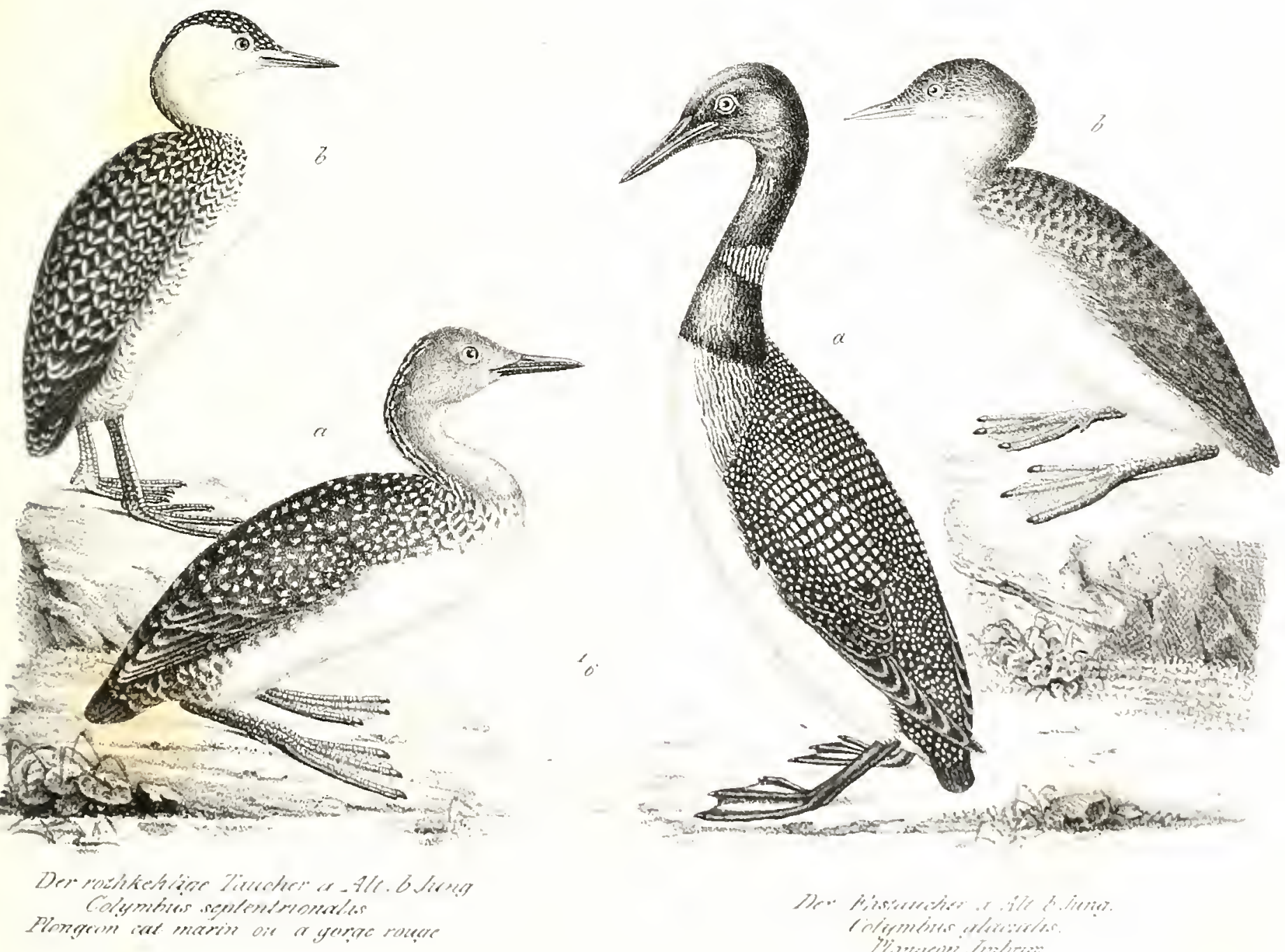

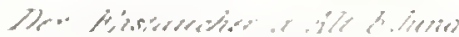




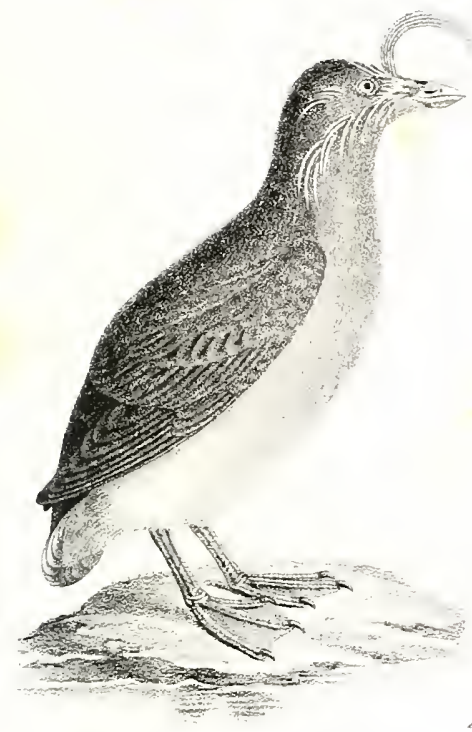

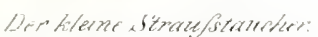
l'halerter piestritiles. Survigur cristatello

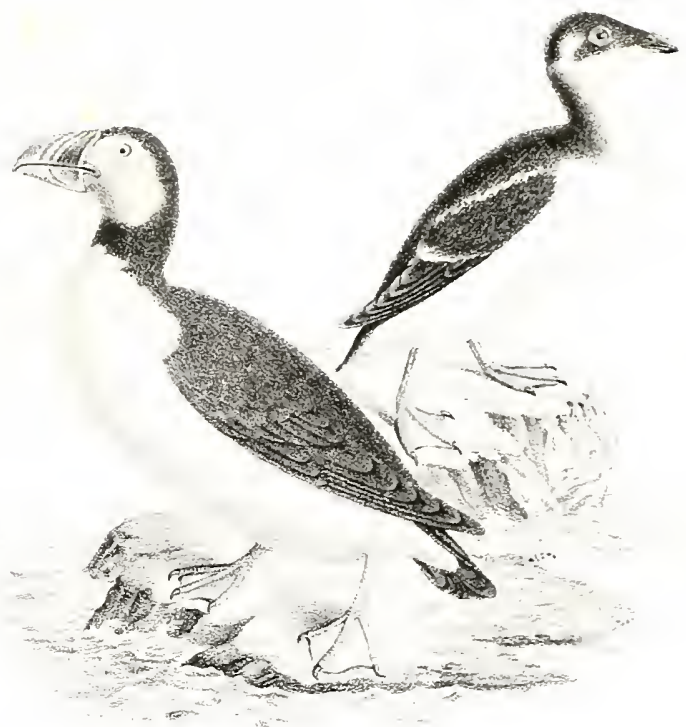

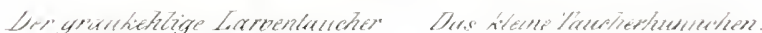

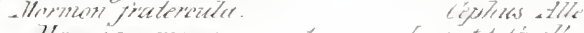

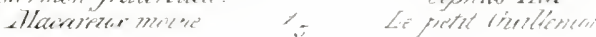
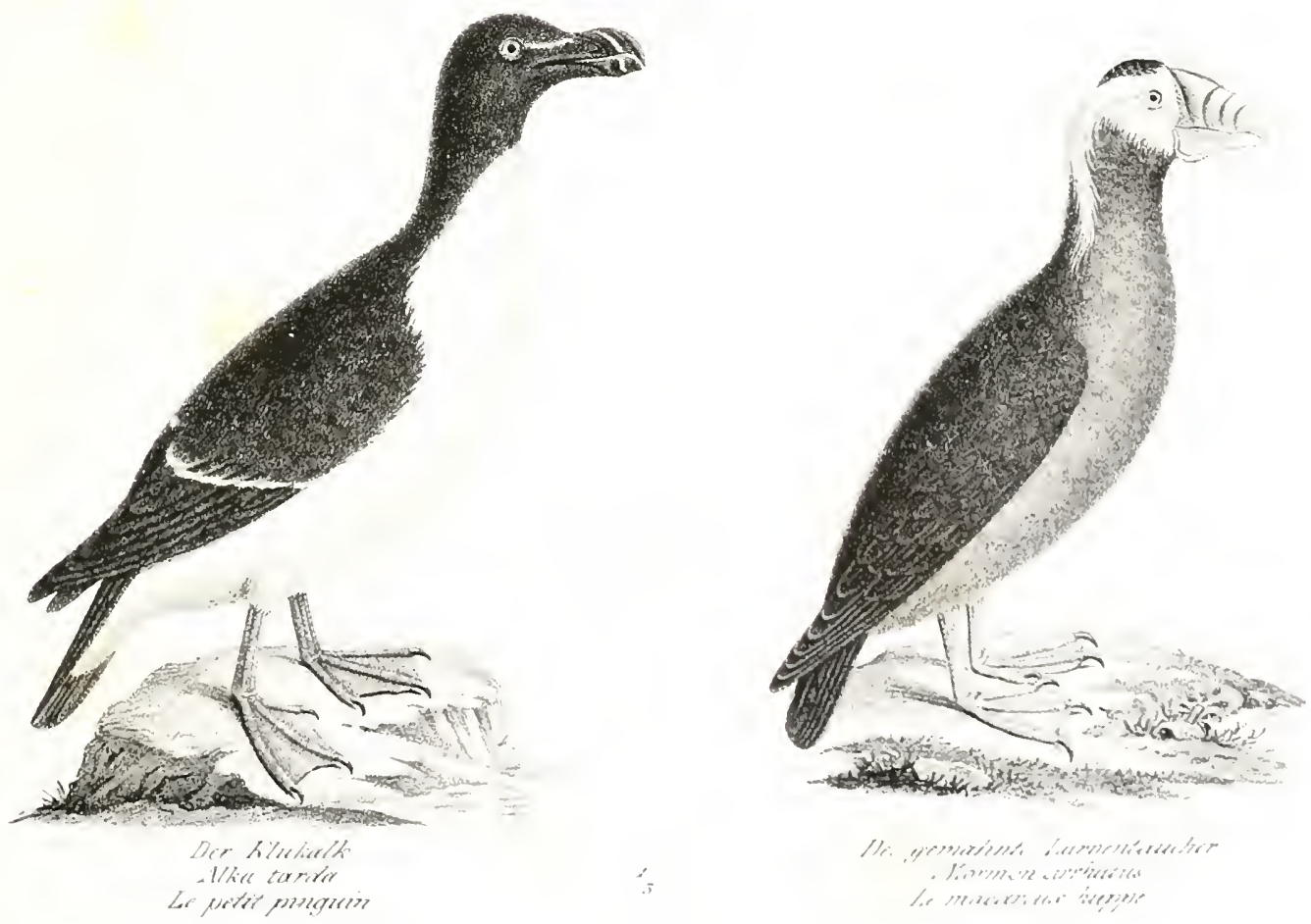

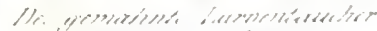





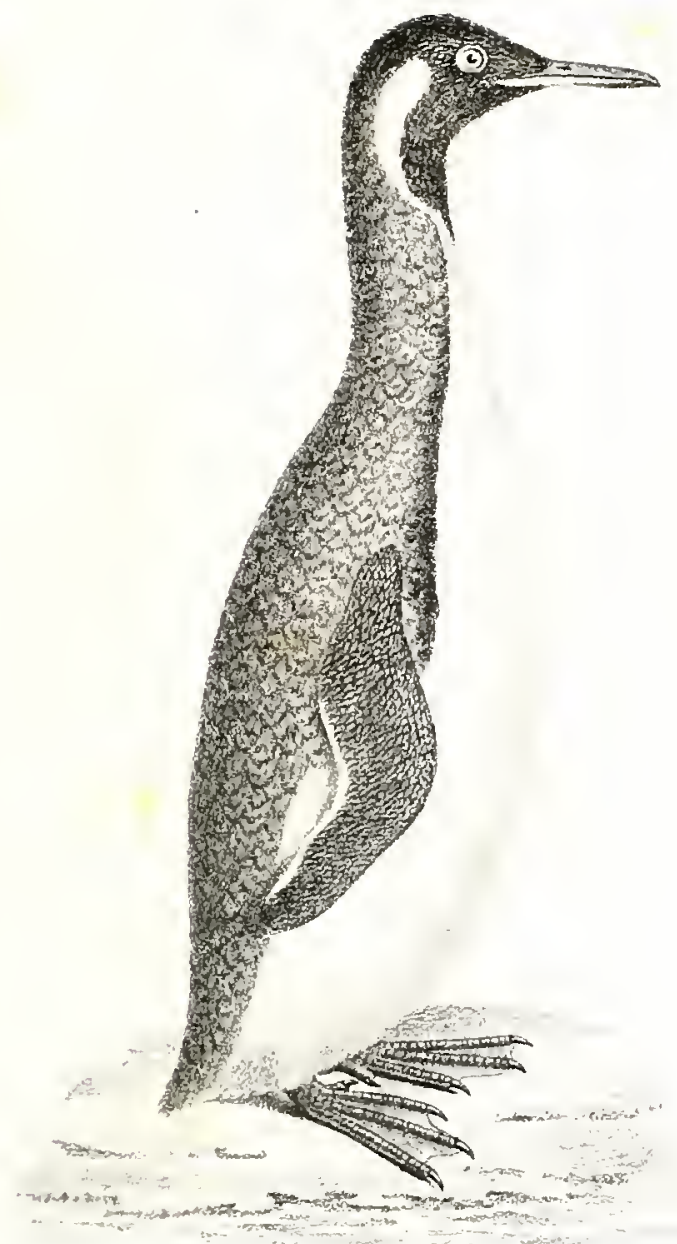

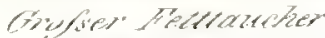

Aptomodites patorroned

Le grand merrebal

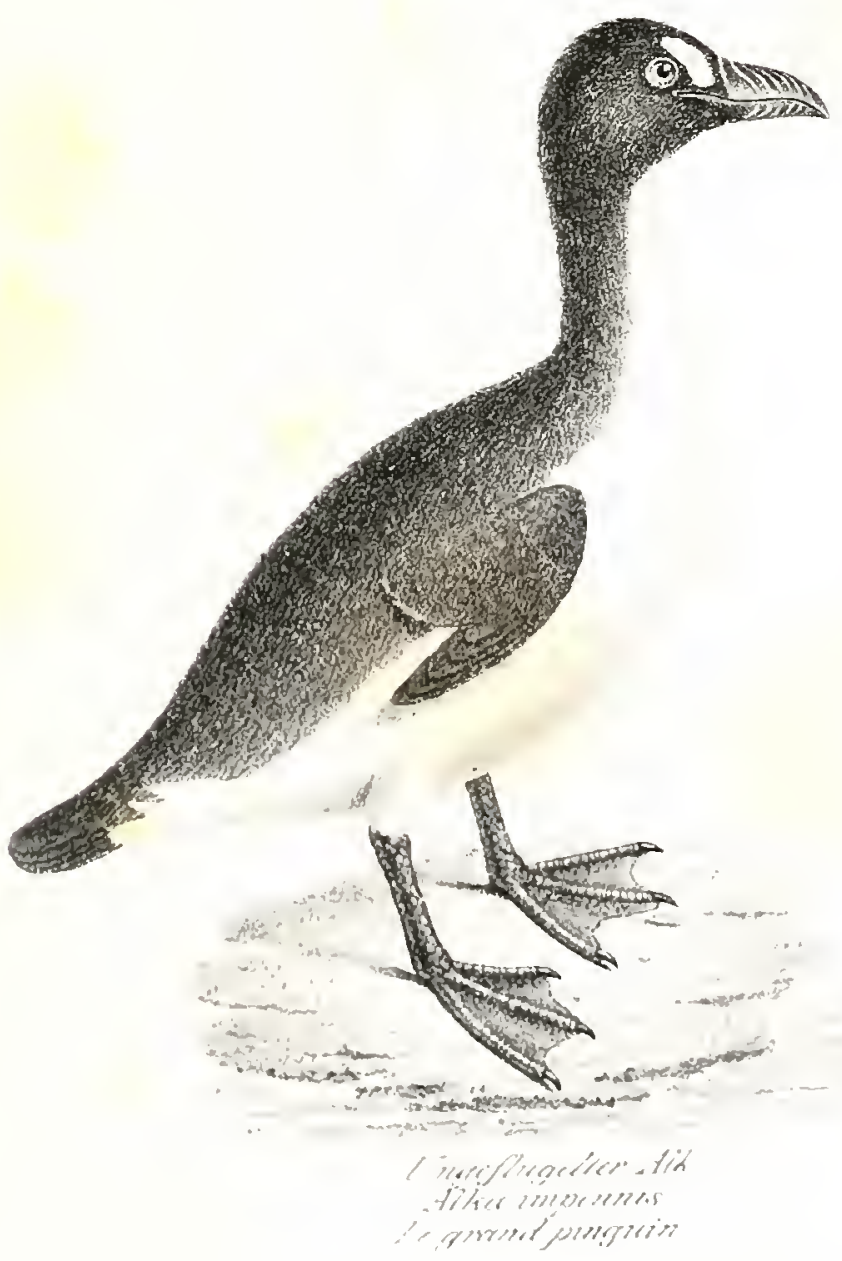

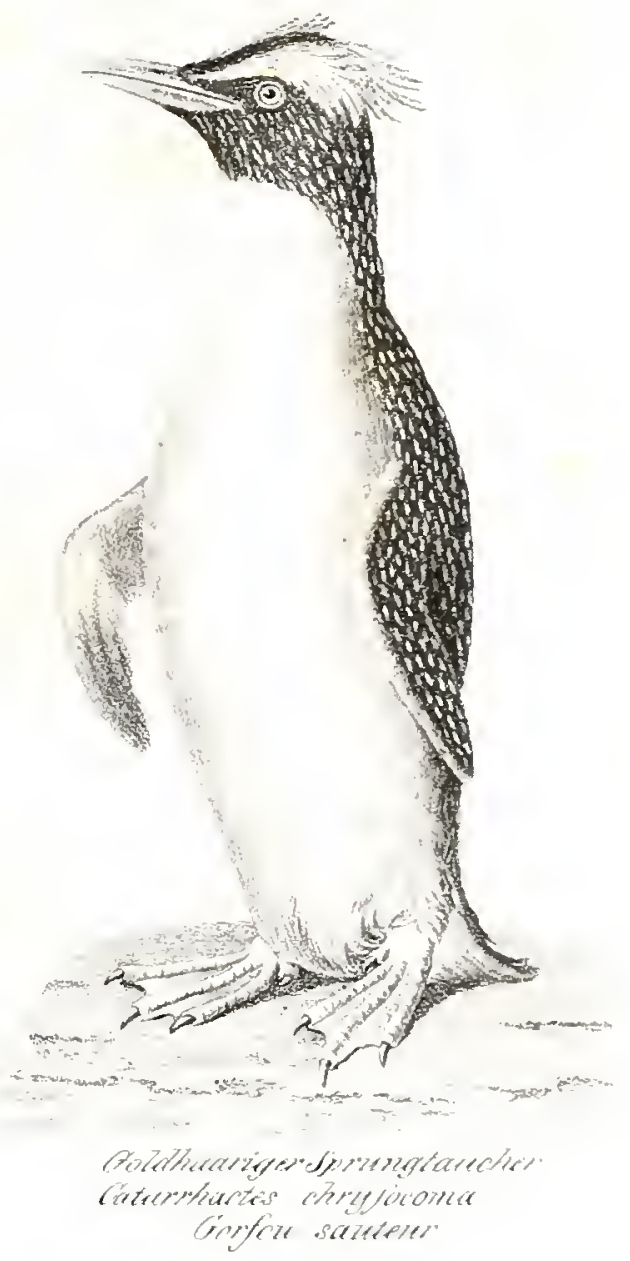
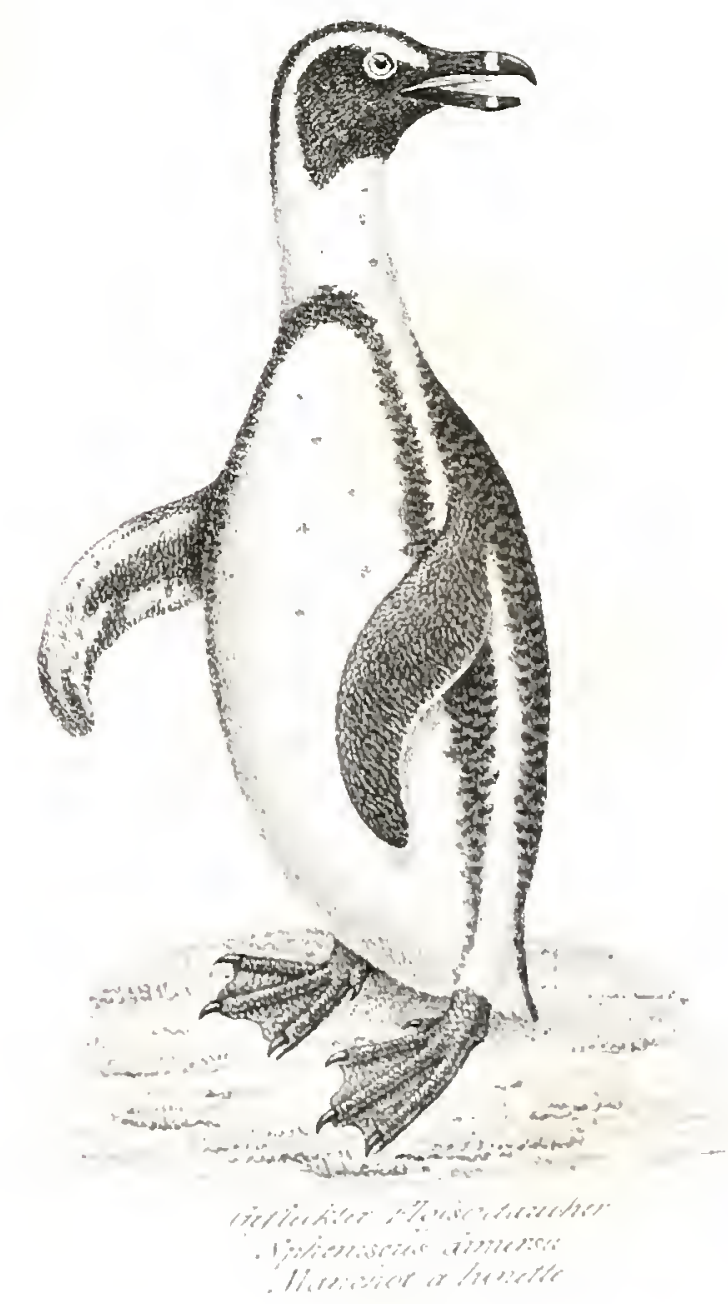


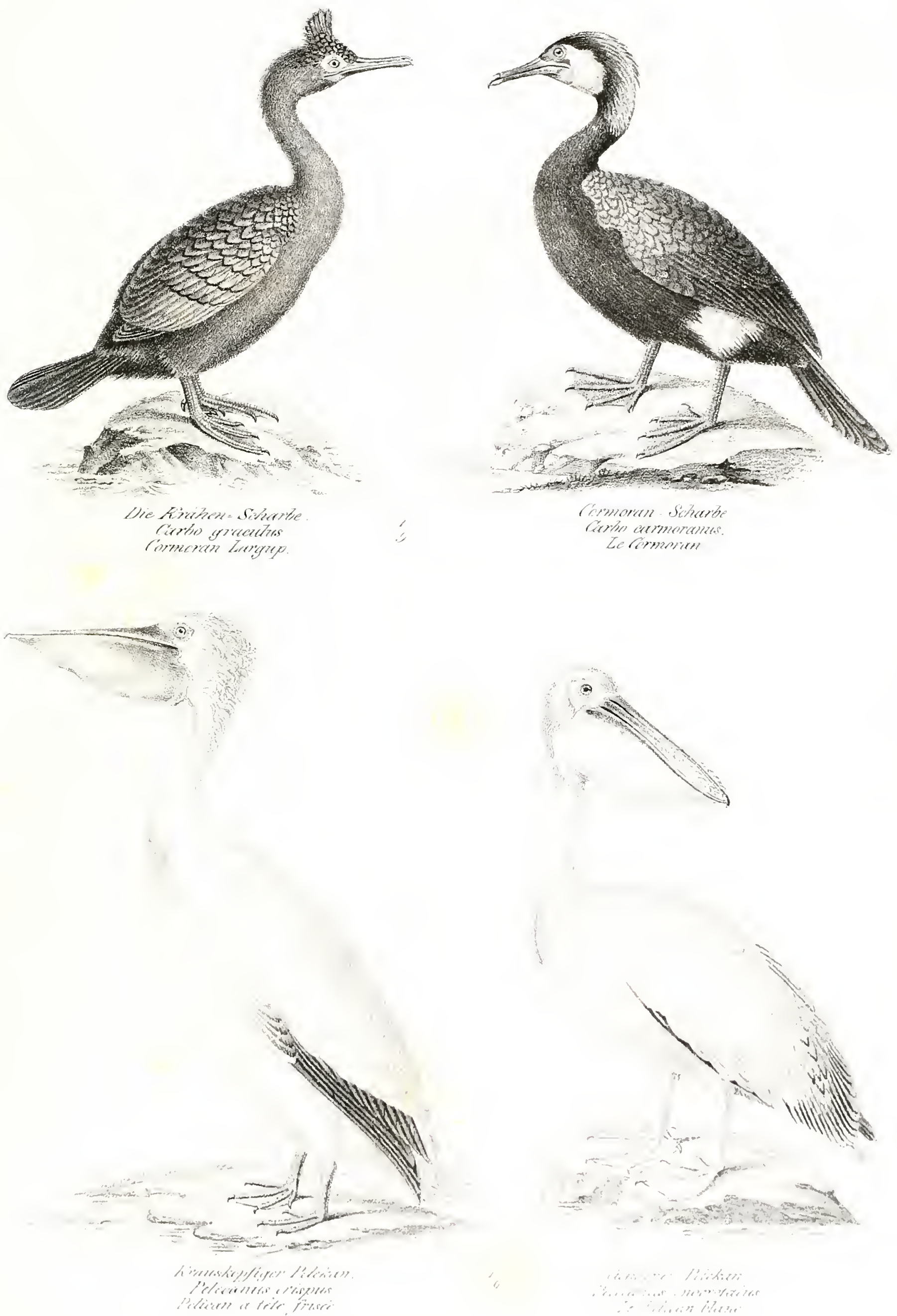


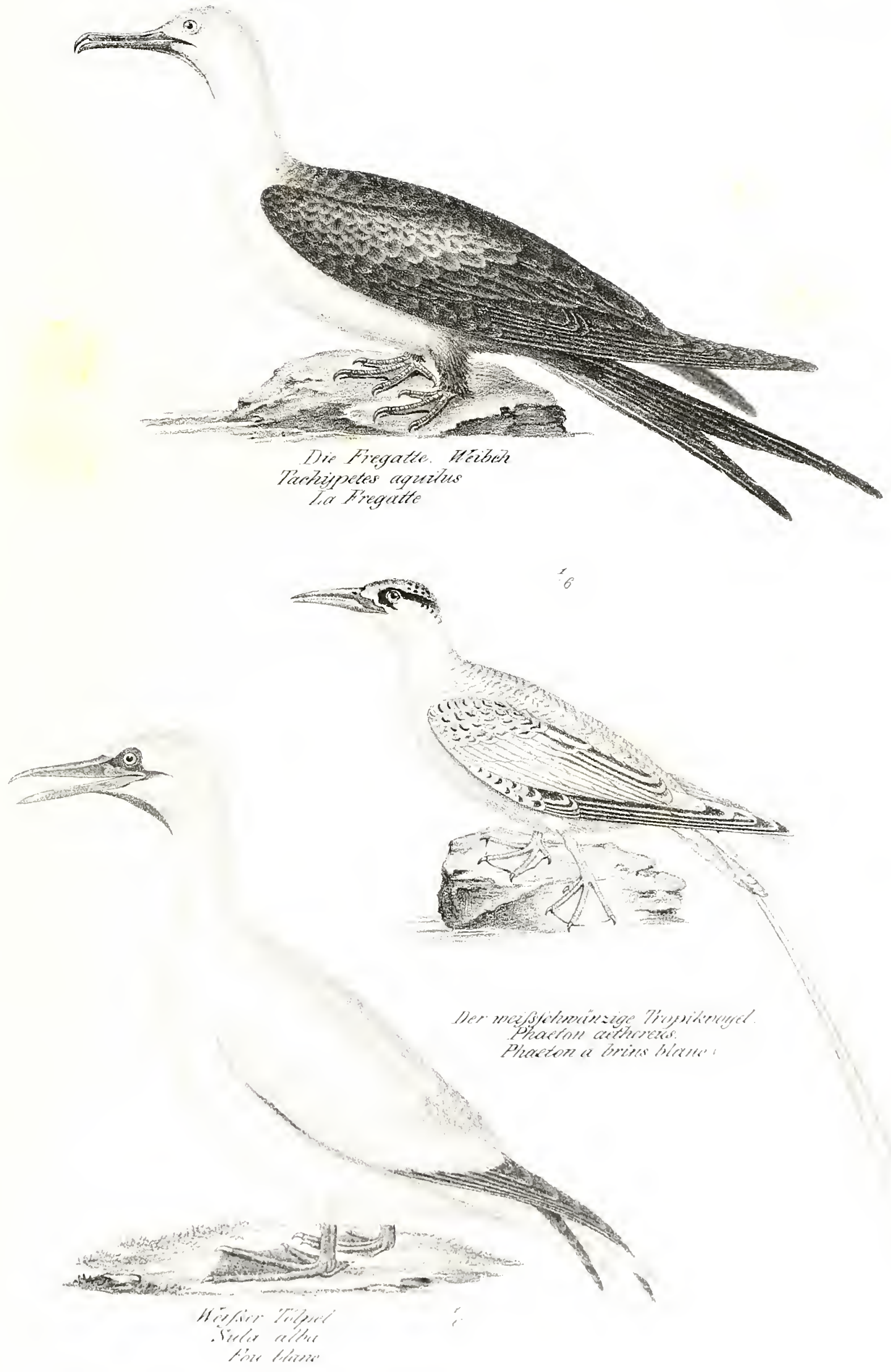

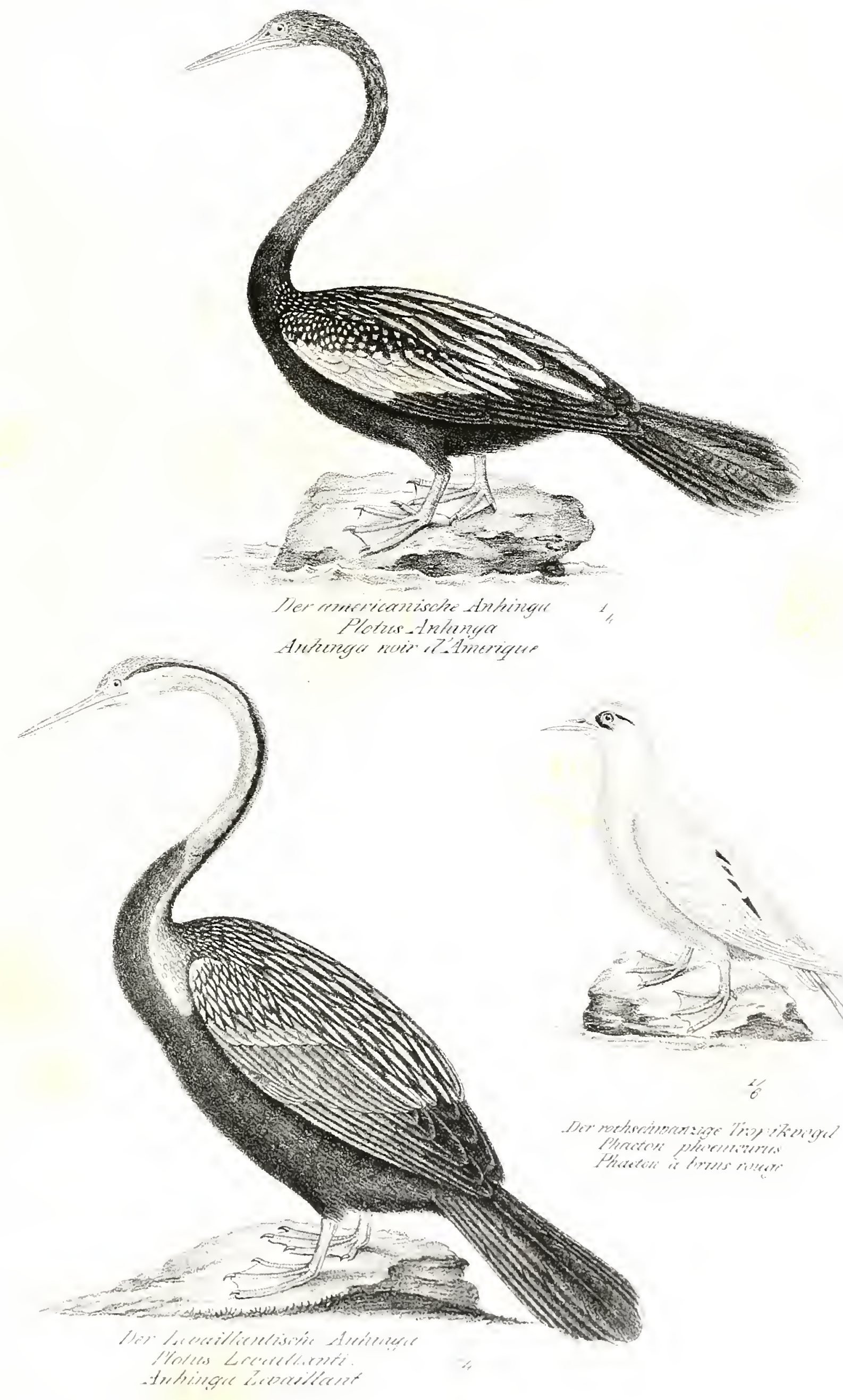


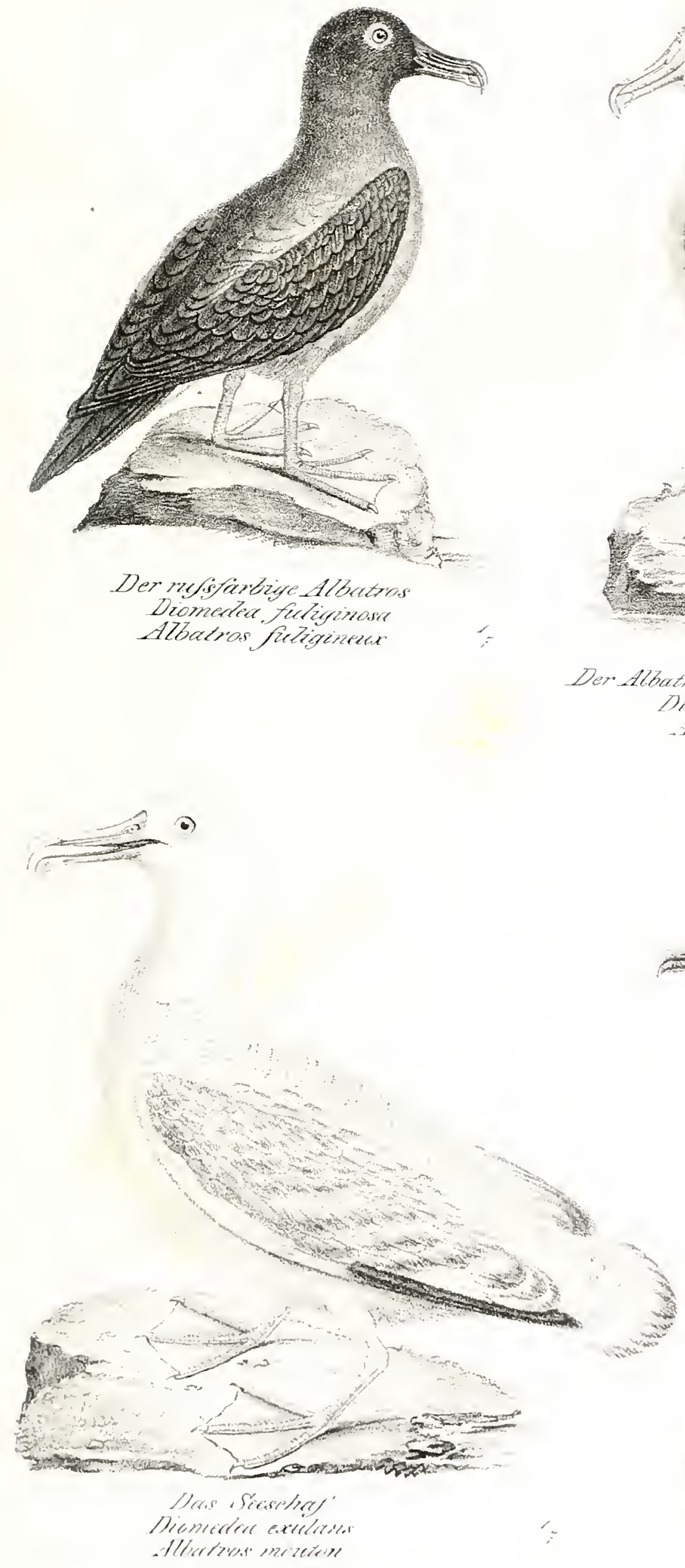

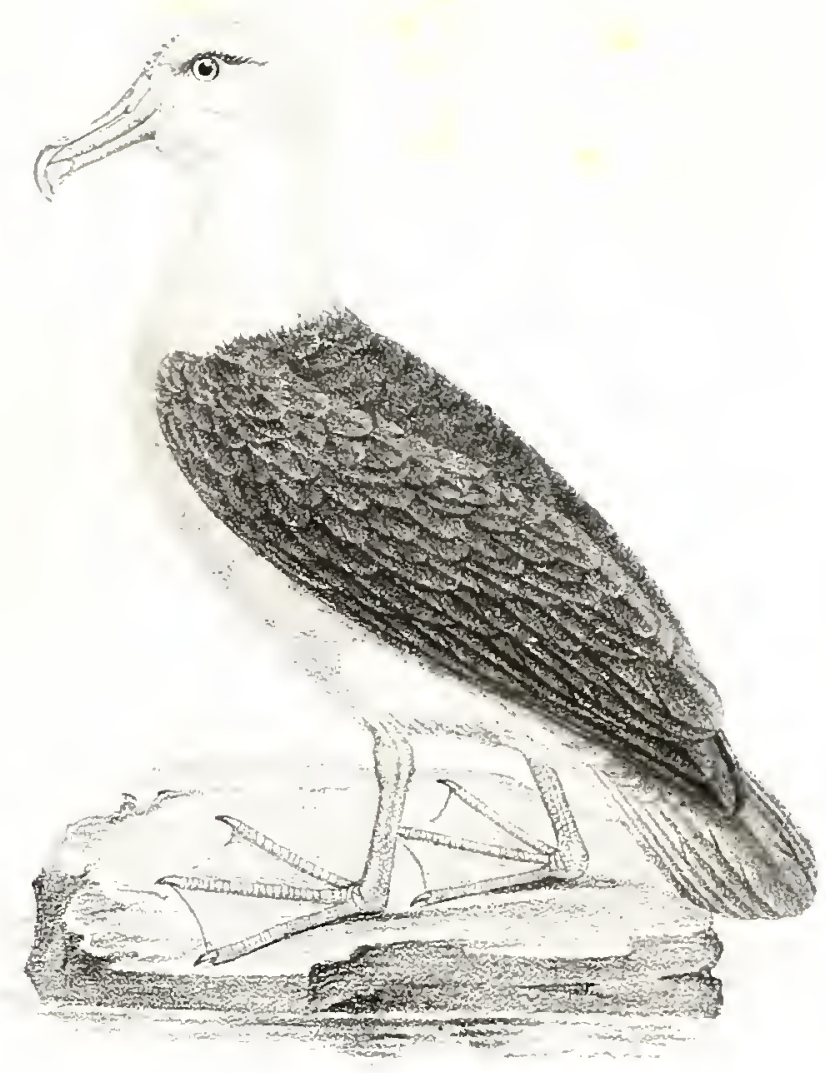

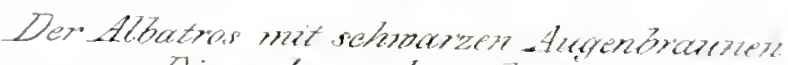
Dimmeder melamophor - Albatios sourcila nazi.
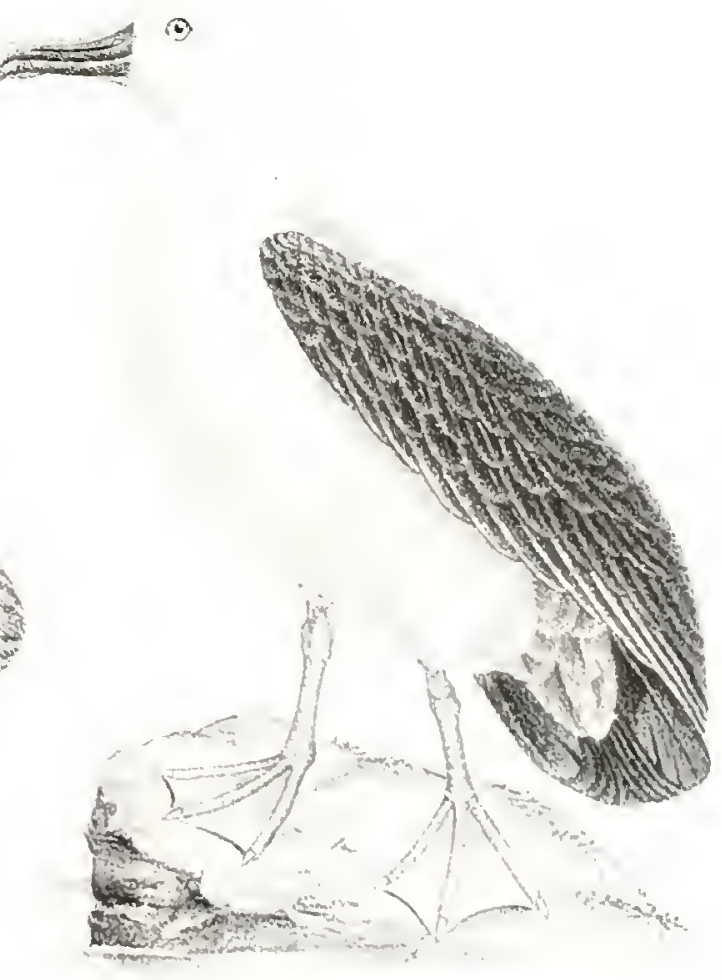

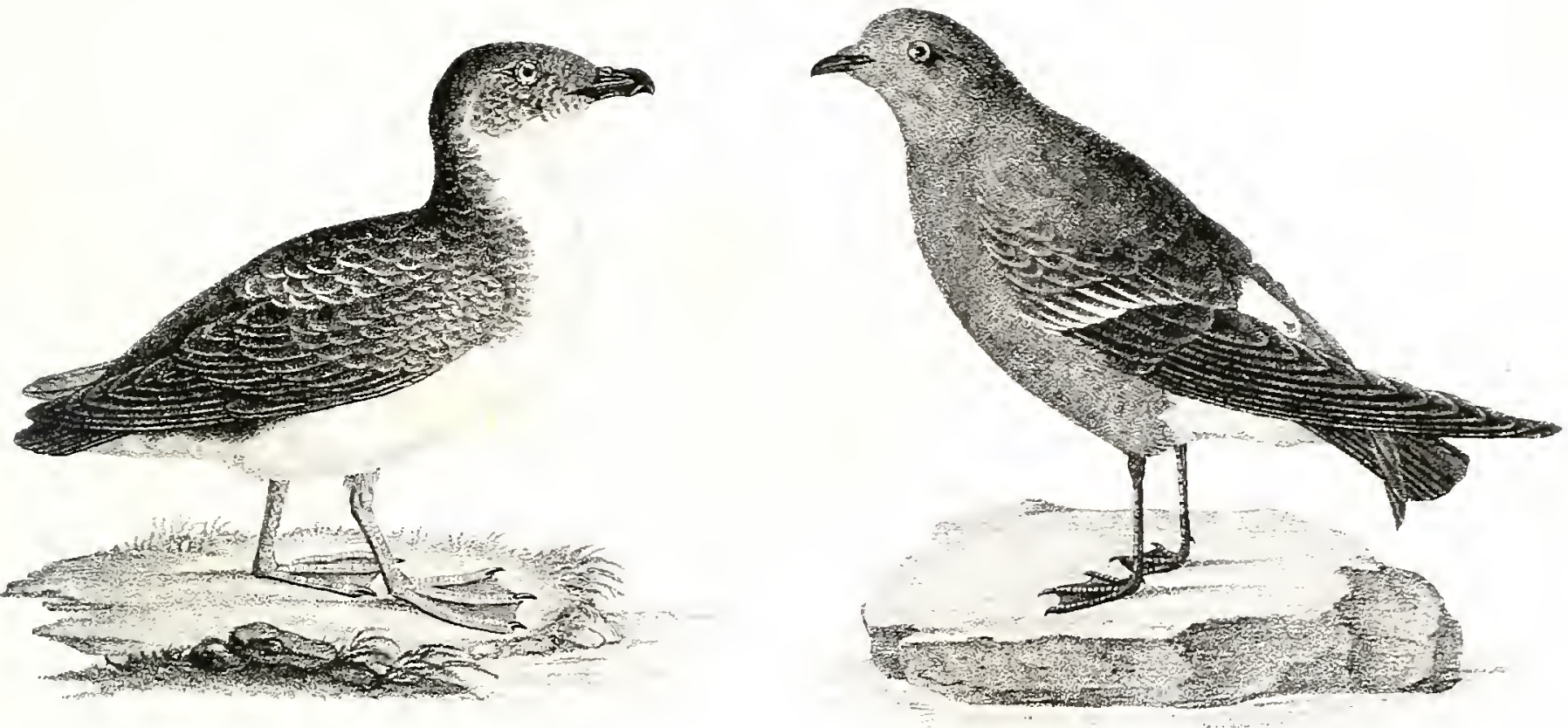

Berardische Starmtermant. Haletroma Berardi.
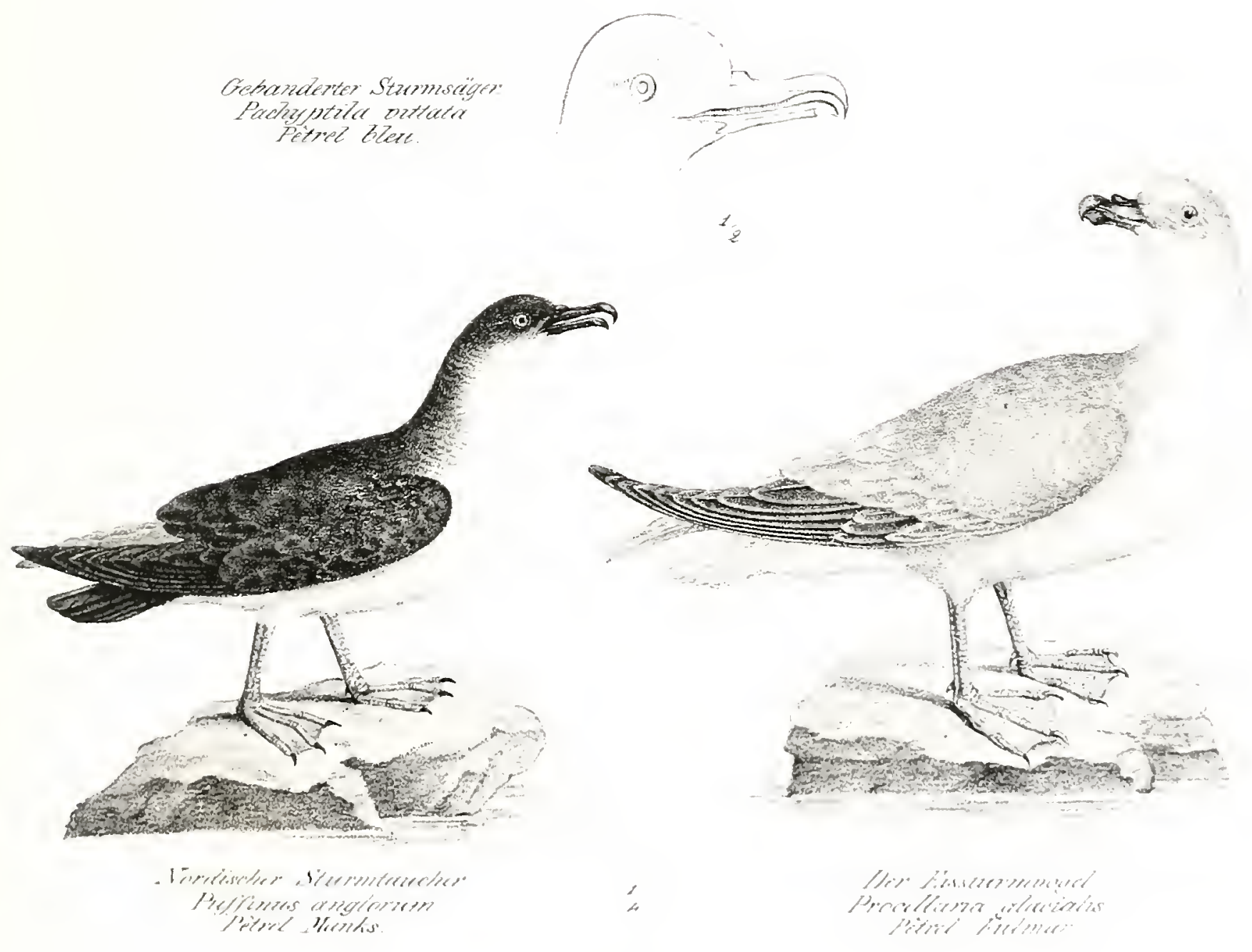

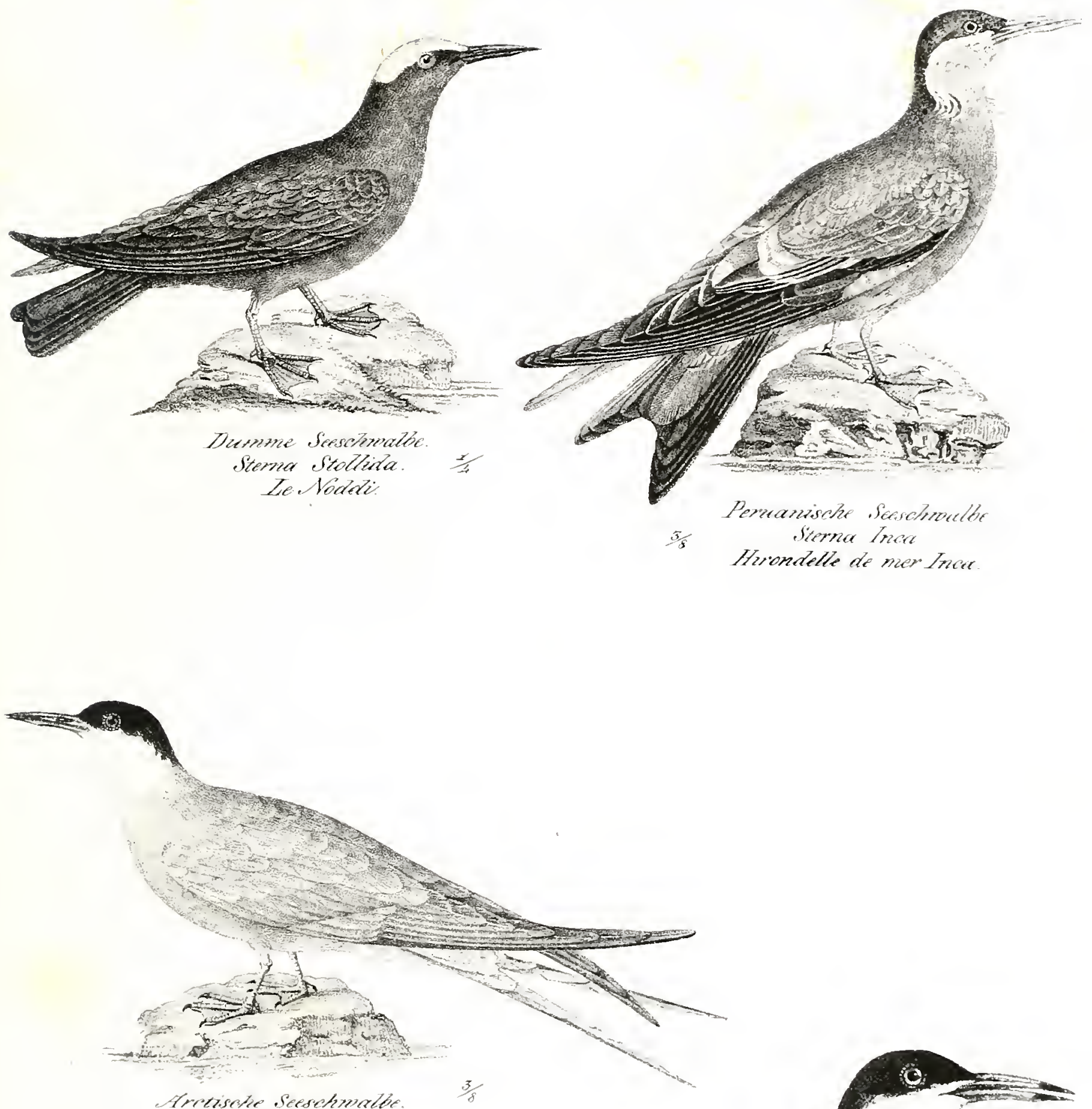

- Syerner rrectaca.

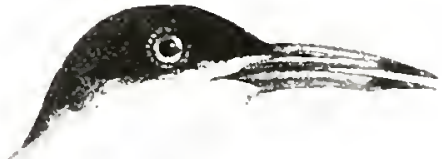

thinndelte ite mer rentigues.
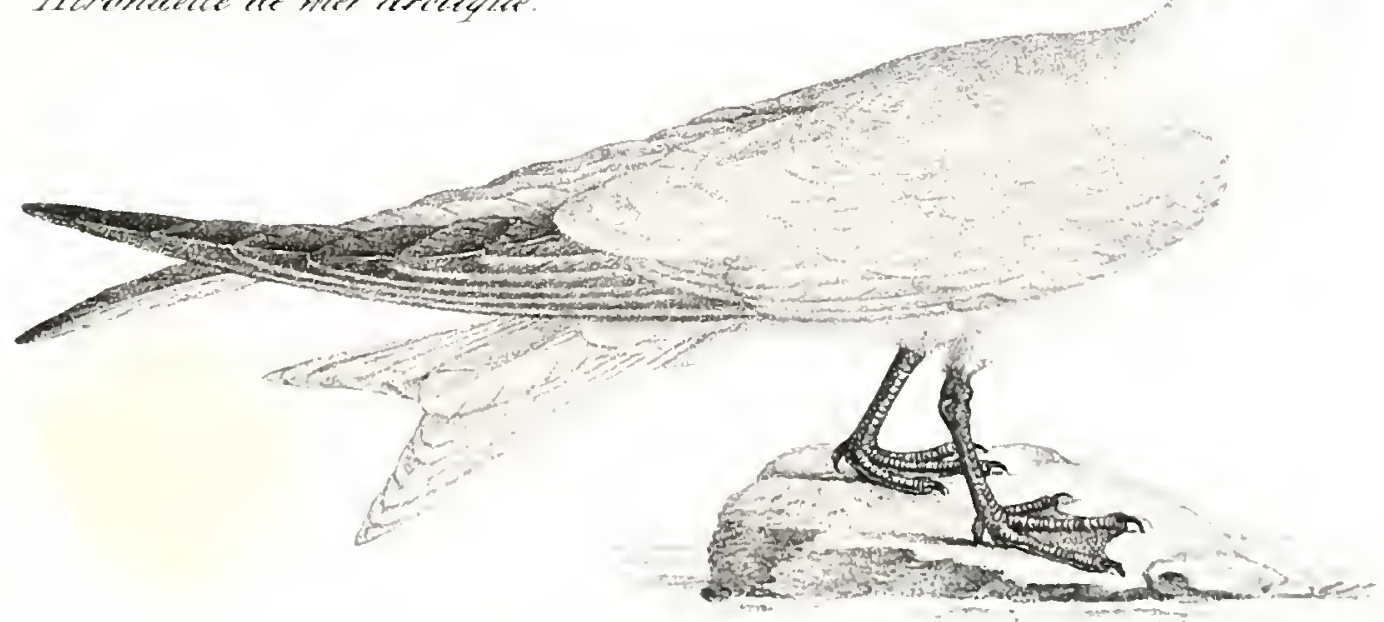

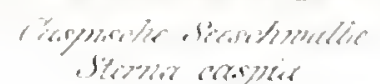

Buroudatie de mere Rechesprat 


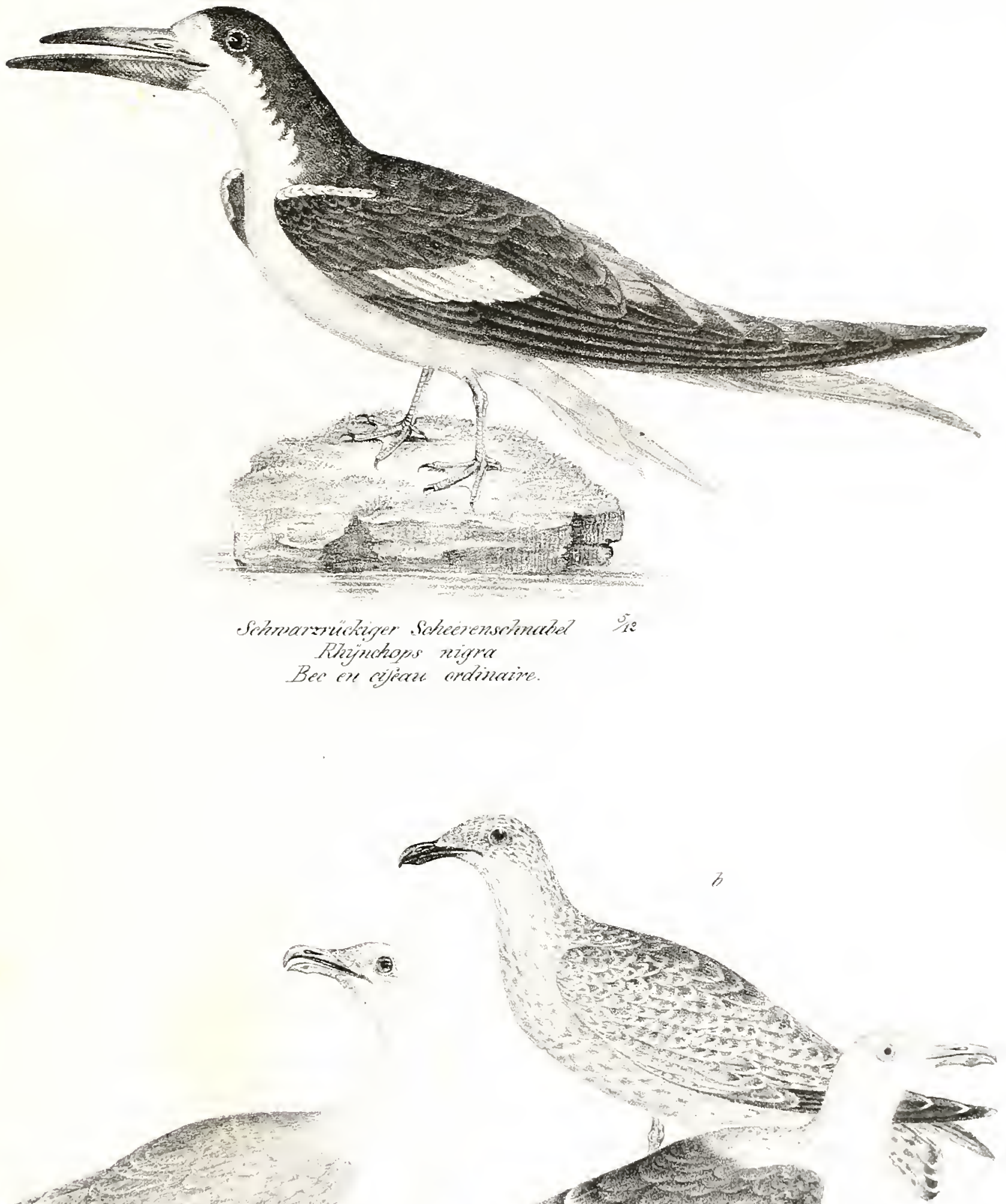

cers 


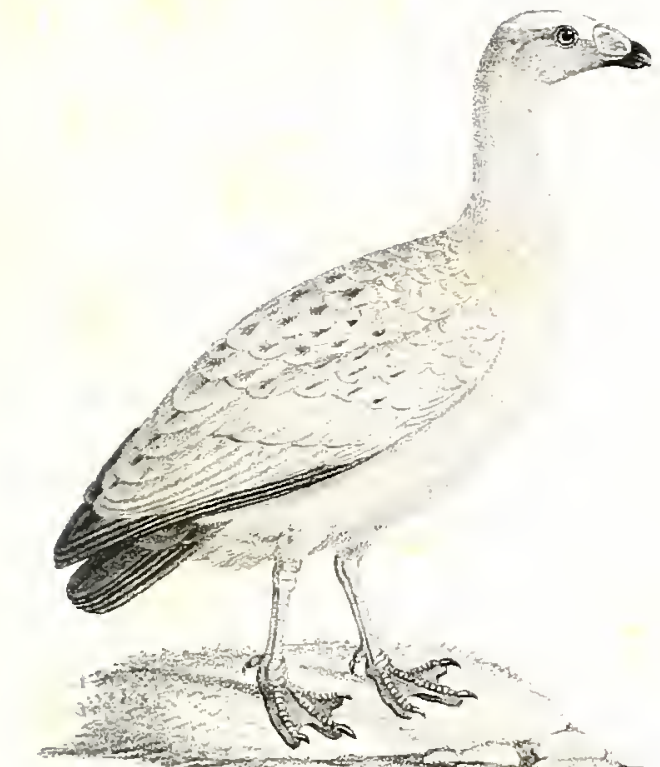

I Der Rappuernovel.

Cereopusis noure Abllowedine. Cénciar ceridie.

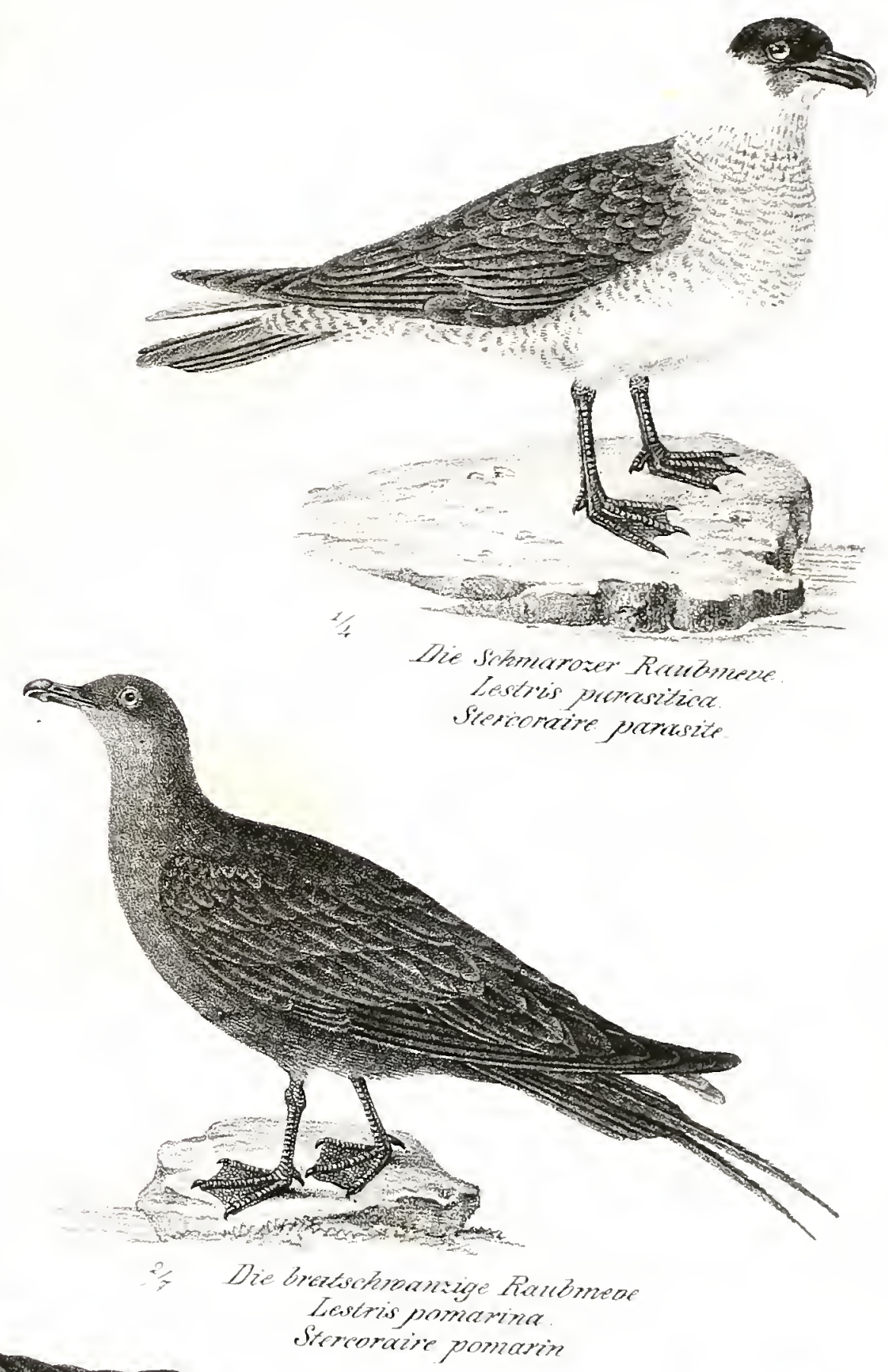

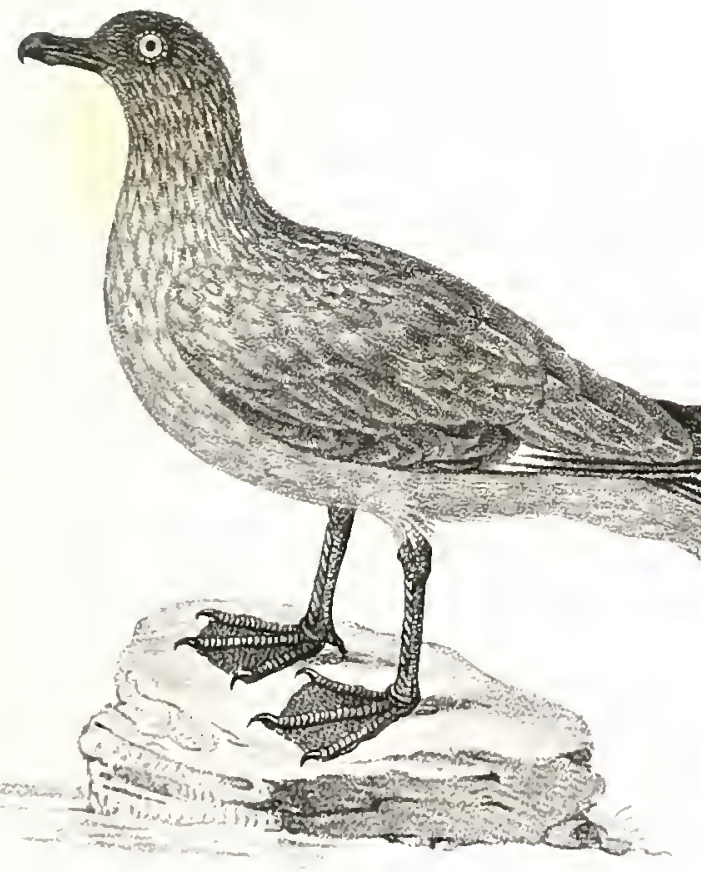

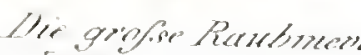
lowistes rovtervacter.

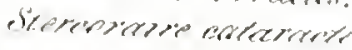

utercoratise porrasin 
140.
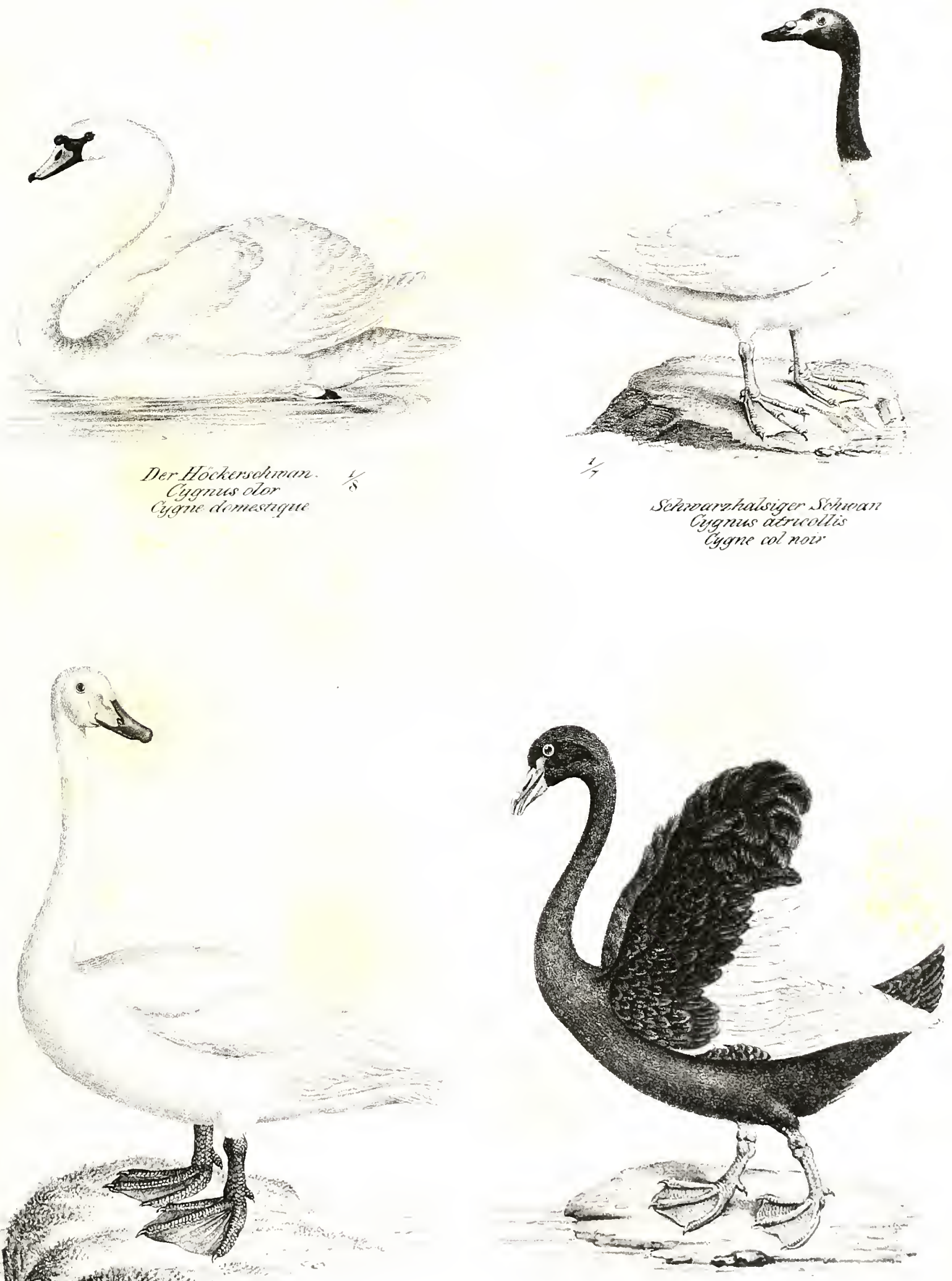



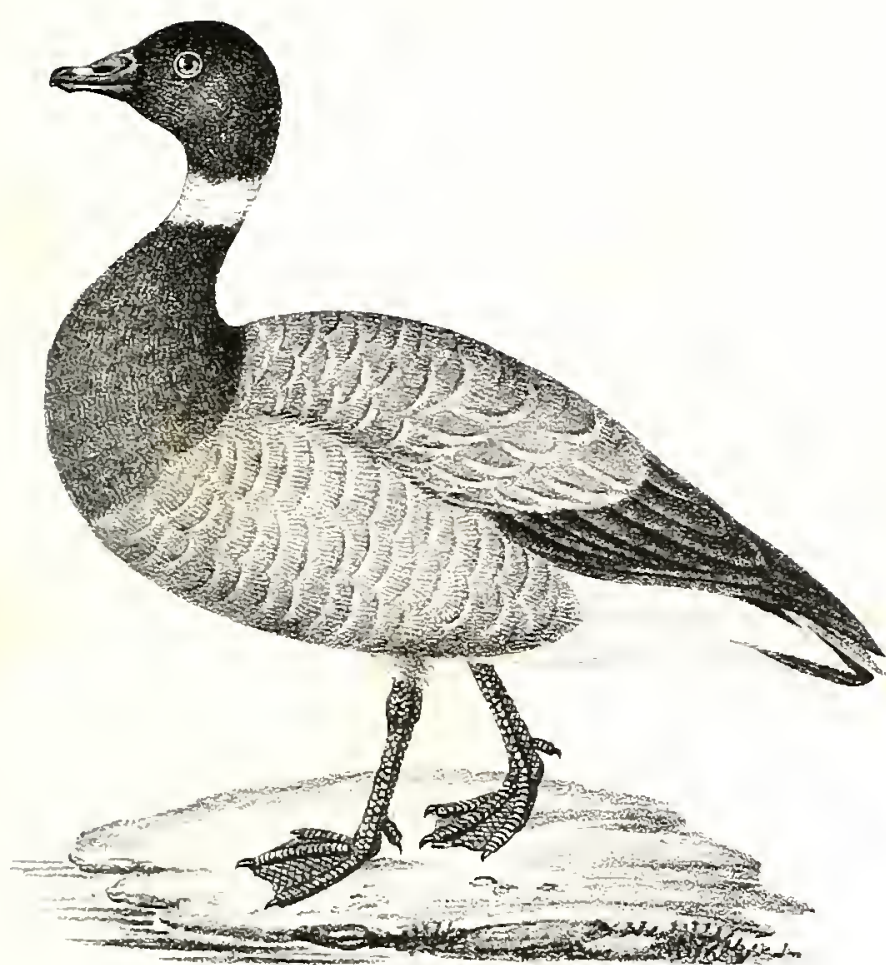

Die Ringelesans

Anuper bertivile

the crazuant.
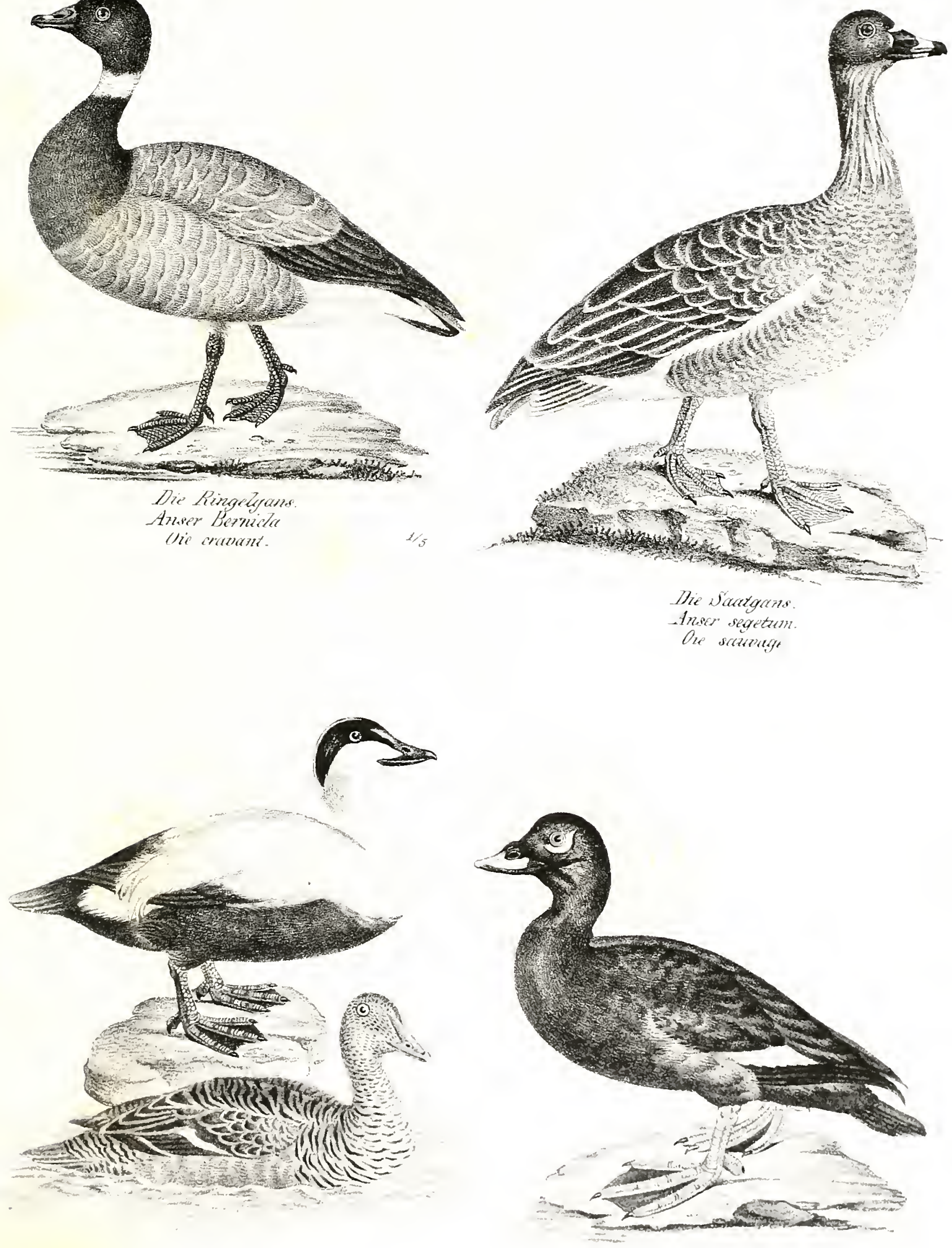

Der limbrome

dinas molefsosiser

Cirmerit linter

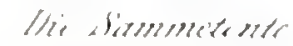

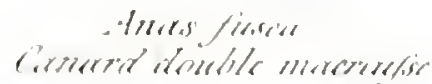

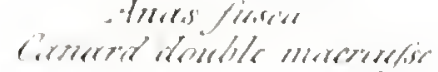





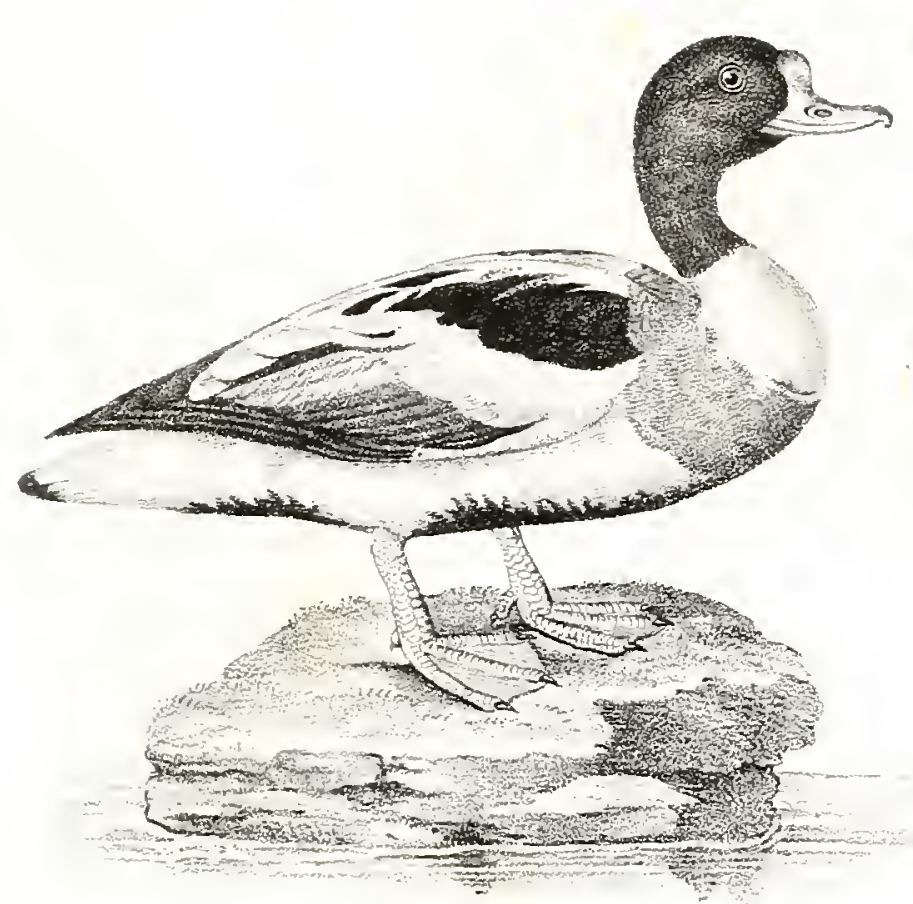

Die Birndente

Ances latorne.

comernt tuderpe.

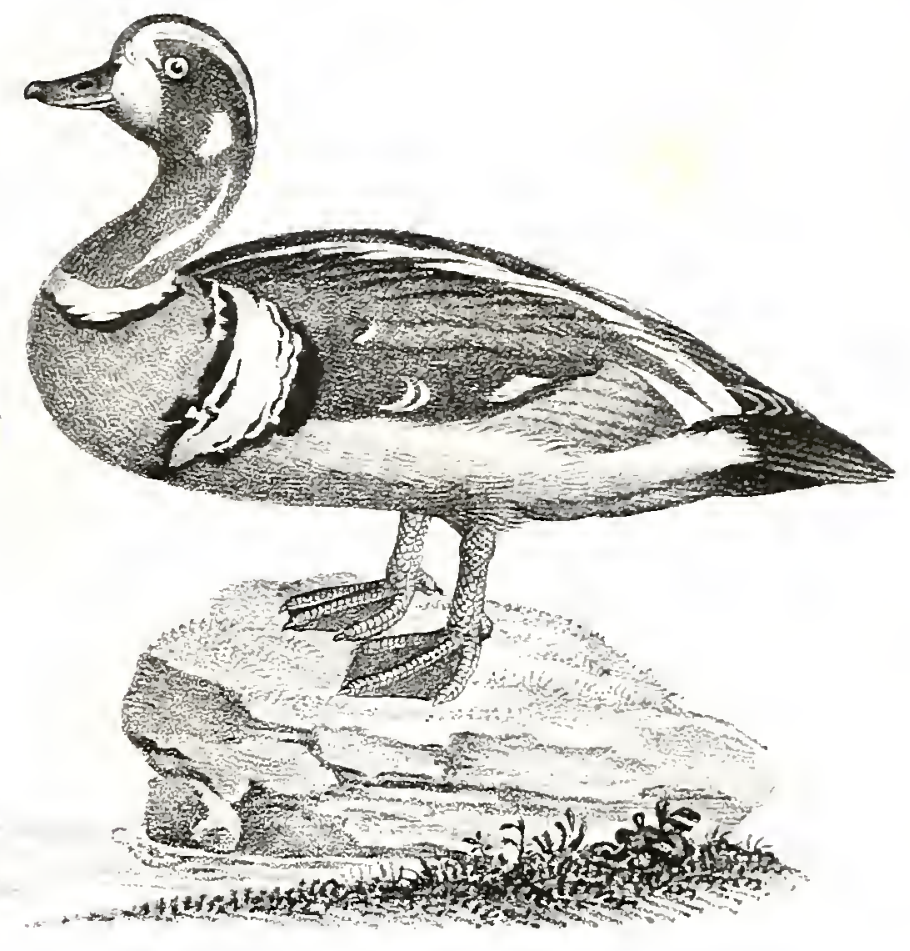

Die hraygereste.

Anres histriculer

Camared id allio.
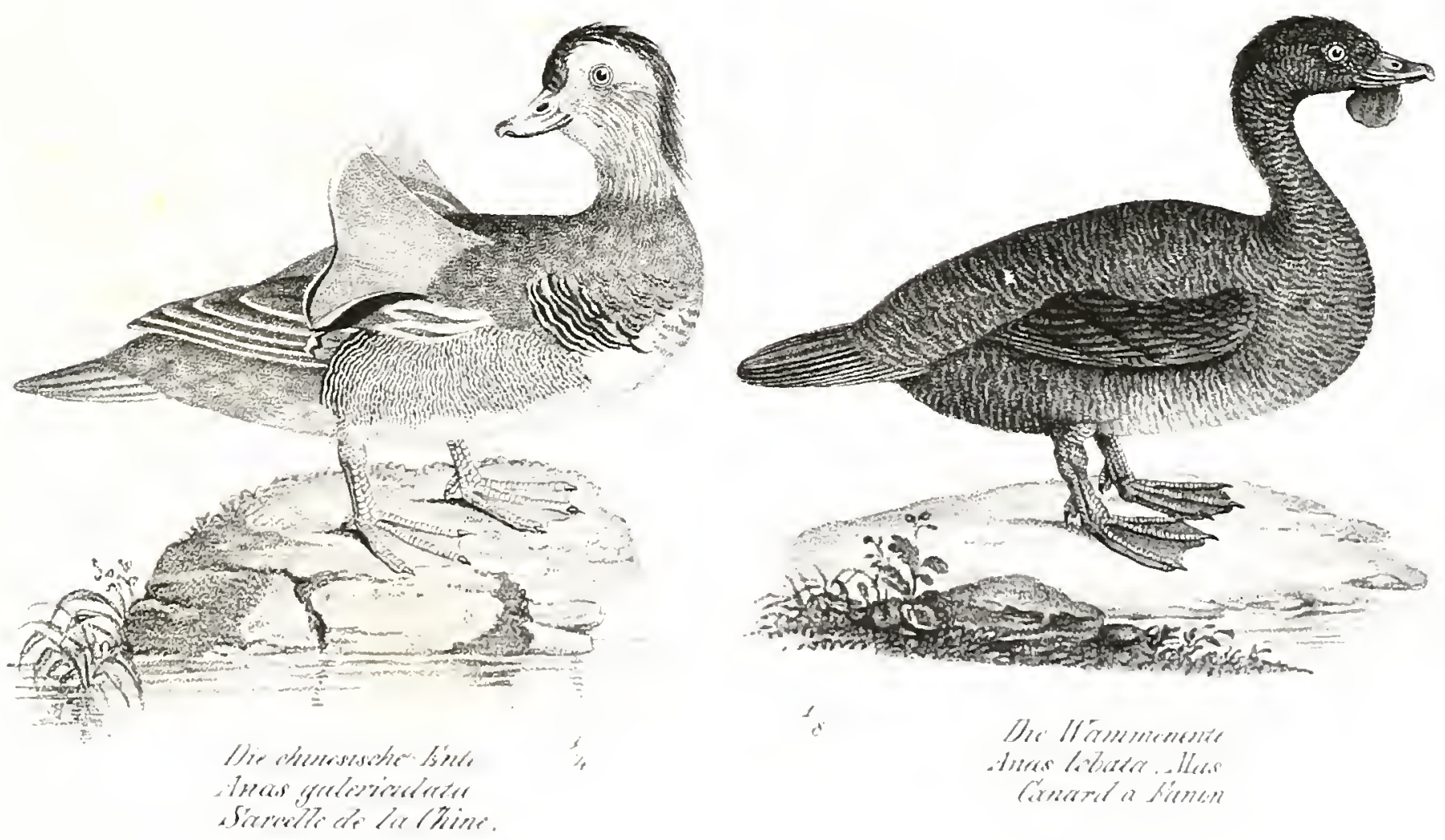

Dhe Hermsmeshent

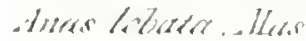

crestorit a fonepl 



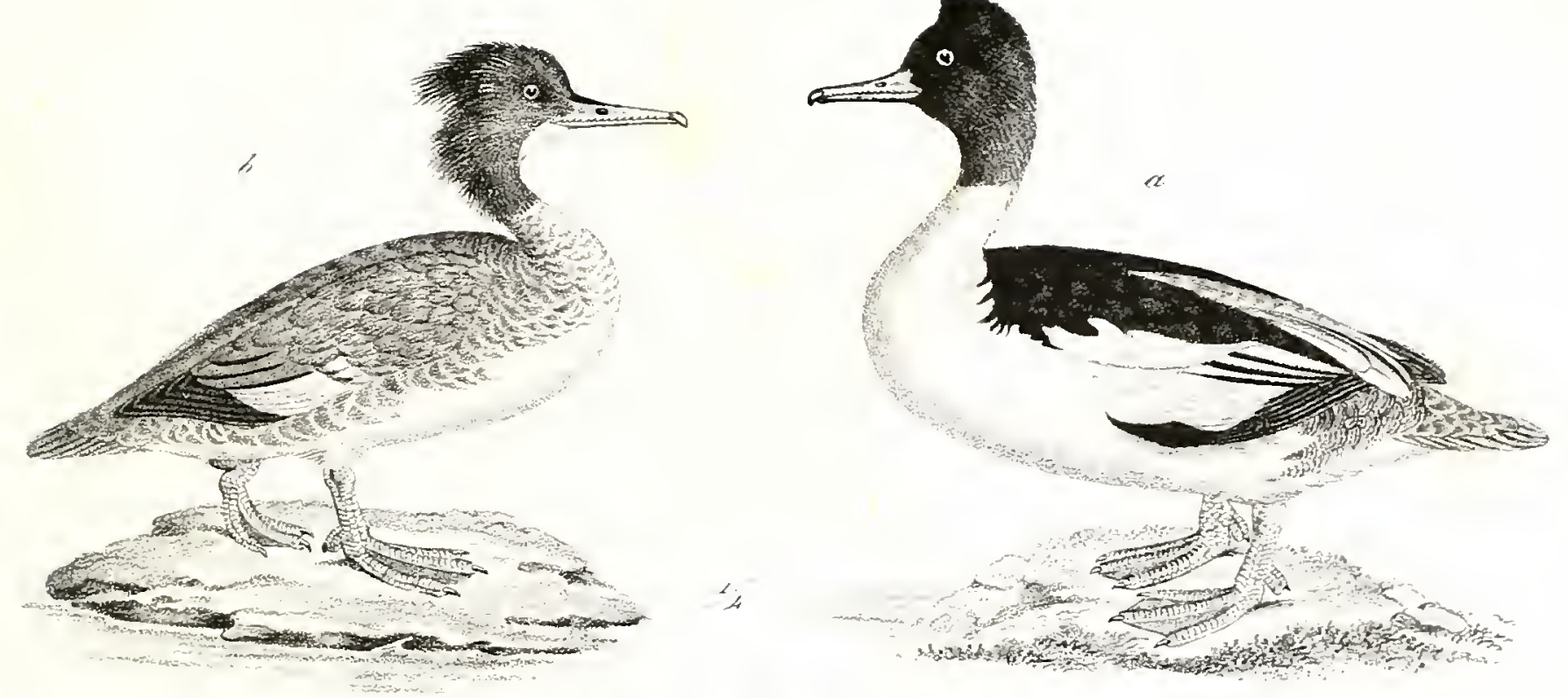

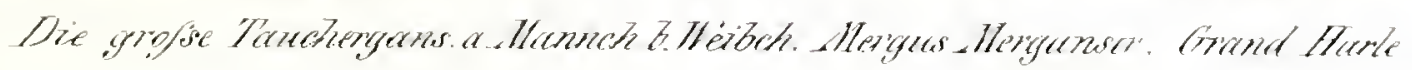

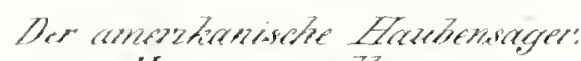

- Heryses mentlatues

Brovle tapepe' d'Amesteres.
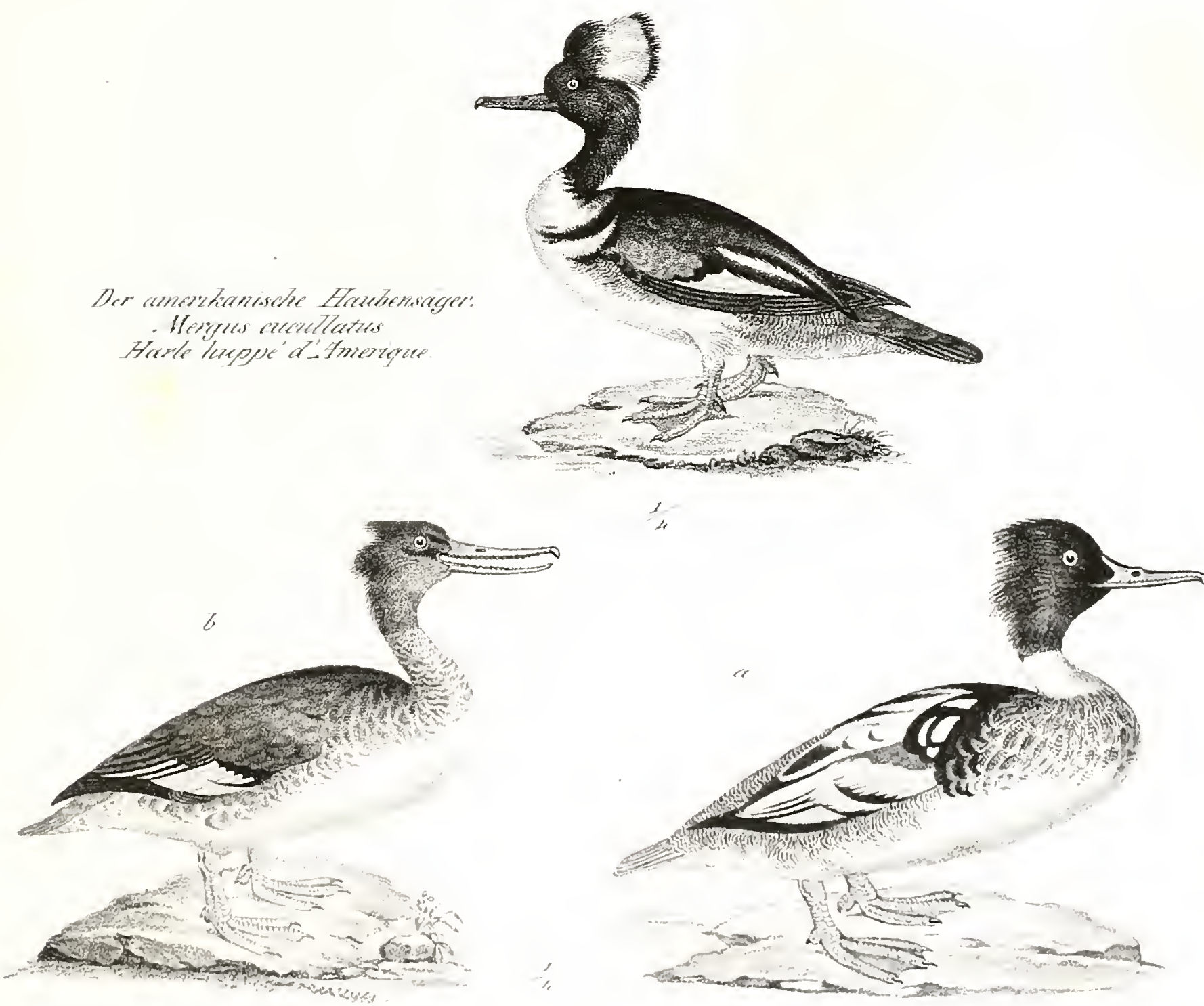

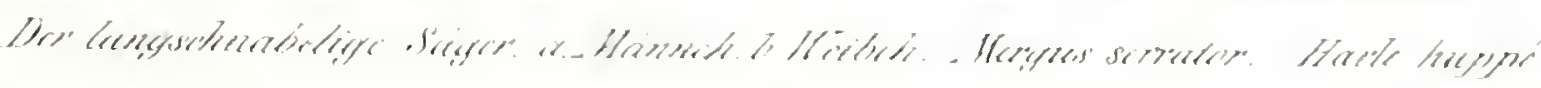




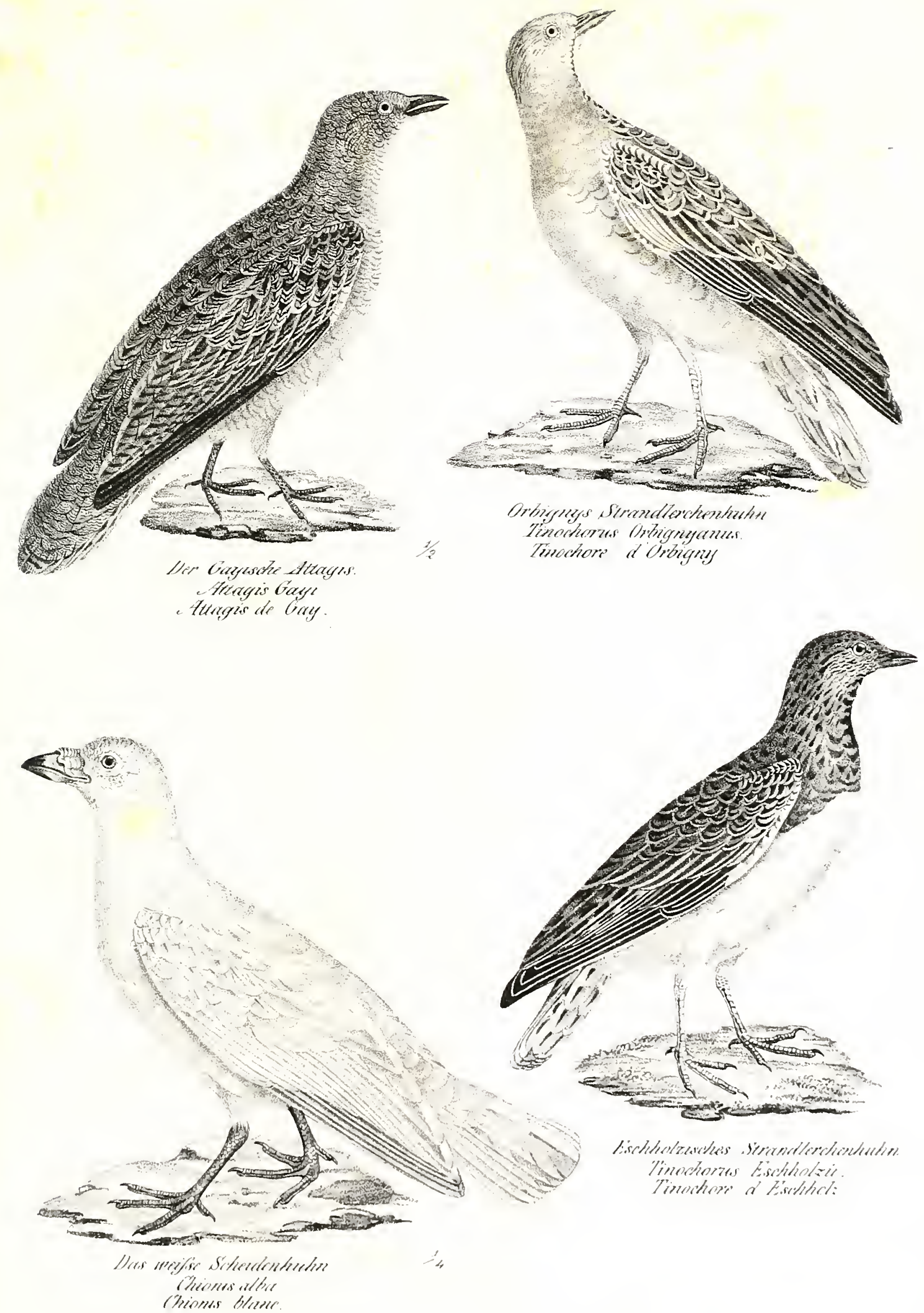



(1) and

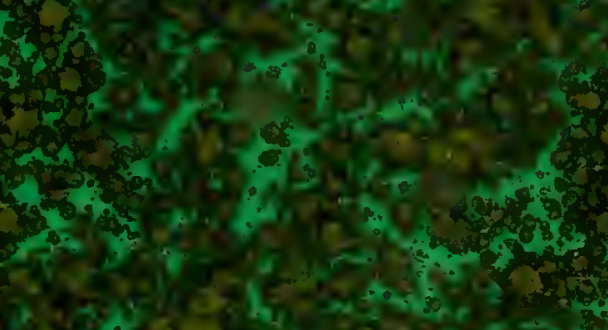

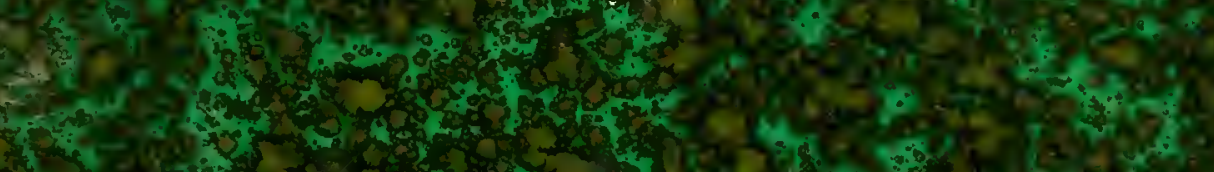

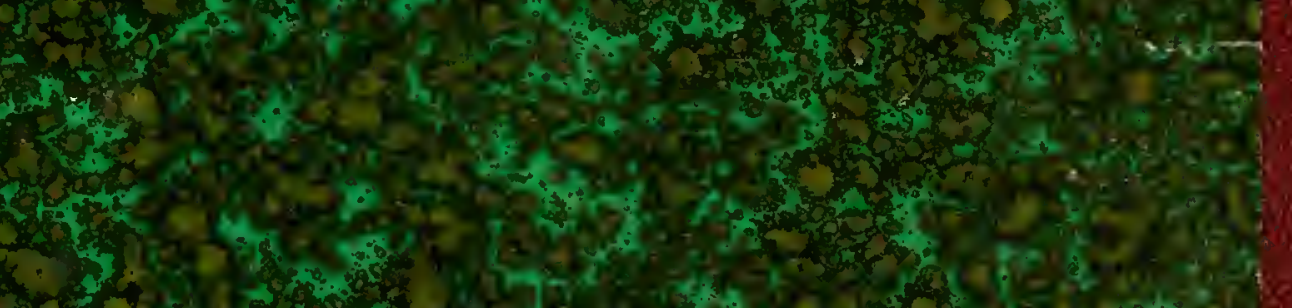

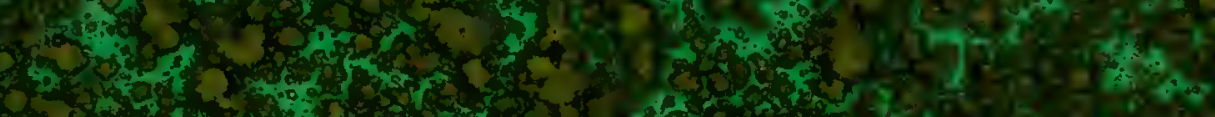

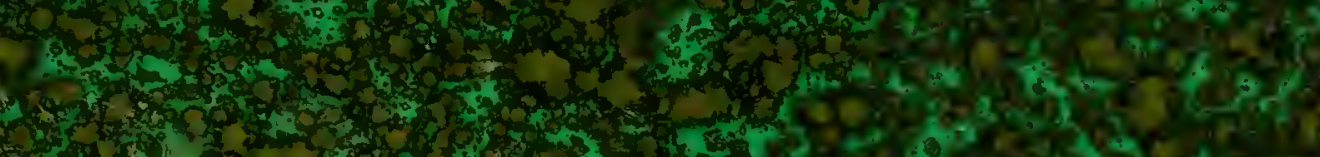
Ant

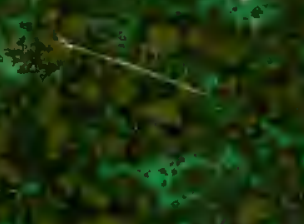
1.

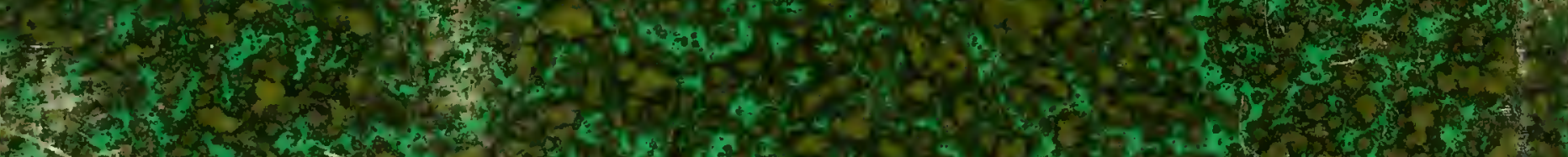

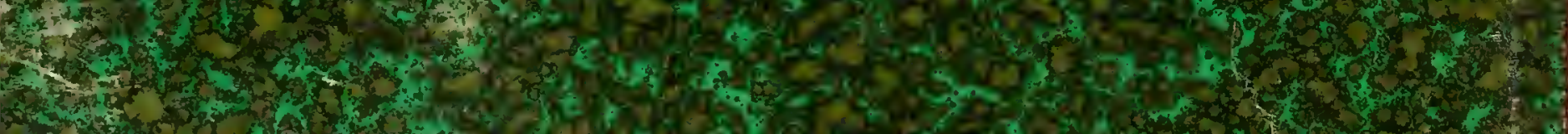

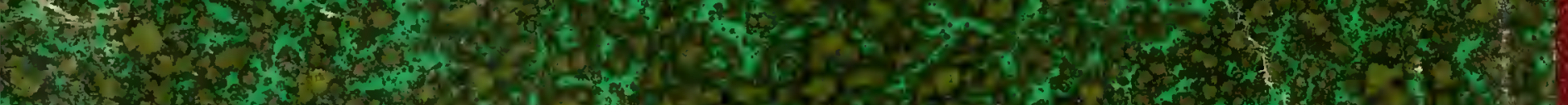

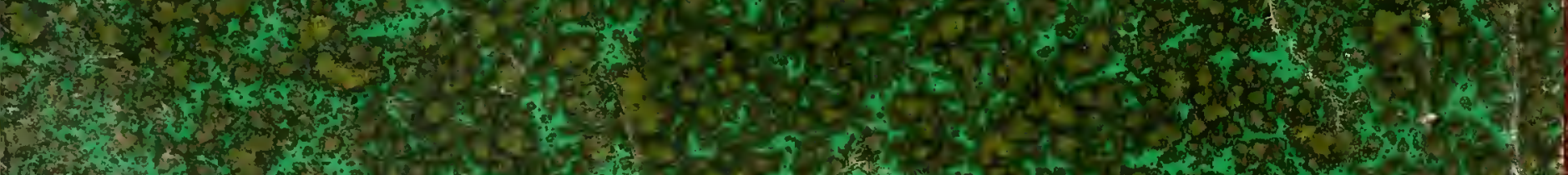

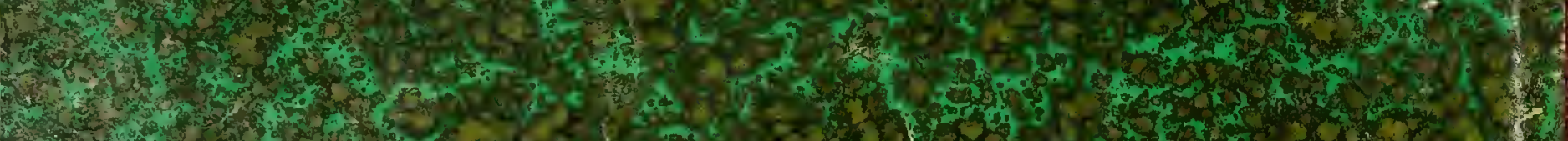

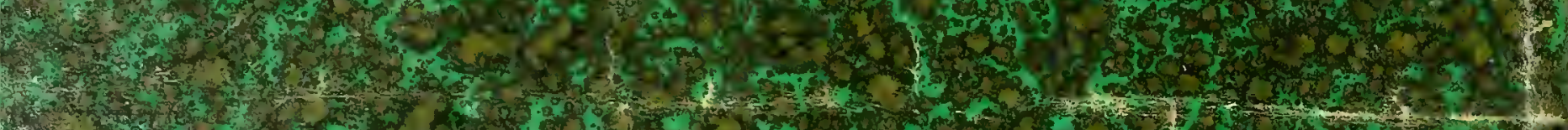

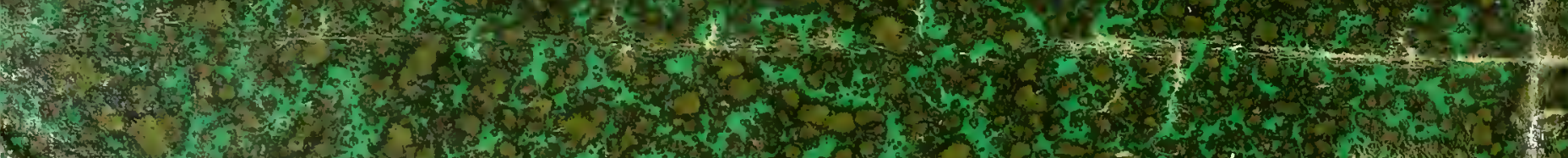
S. 